\title{
Hiper-Ideais de Aplicações Multilineares e Polinômios Homogêneos em Espaços de Banach
}

\author{
Ewerton Ribeiro Torres
}

TESE APRESENTADA

$\mathrm{AO}$

Instituto DE MATemática e Estatística

DA

Universidade DE SÃo Paulo

PARA

OBTENÇÃO DO TÍTULO

$\mathrm{DE}$

Doutor EM CIÊNCIAS

Programa: Matemática

Orientadora: Prof ${ }^{\mathrm{a}}$. Dr ${ }^{\mathrm{a}}$. Mary Lilian Lourenço

Coorientador: Prof. Dr. Geraldo Márcio de Azevedo Botelho

Durante o desenvolvimento deste trabalho o autor recebeu parcialmente auxílio financeiro da CAPES

São Paulo, maio de 2015 


\title{
Hiper-Ideais de Aplicações Multilineares e Polinômios Homogêneos em Espaços de Banach
}

\author{
Esta versão da tese contém as correções e alterações sugeridas \\ pela Comissão Julgadora durante a defesa da versão original do trabalho, \\ realizada em 24/04/2015. Uma cópia da versão original está disponível no \\ Instituto de Matemática e Estatística da Universidade de São Paulo.
}

Comissão Julgadora:

- Prof. Dr. Geraldo Márcio de Azevedo Botelho (coorientador) - FAMATUFU

- Prof. Dr. Daniel Marinho Pellegrino - DM-UFPB

- Prof. Dr. Vinícius Vieira Fávaro - FAMAT-UFU

- Prof. Dr. Jorge Tulio Mujica Ascui - IMECC-UNICAMP

- Prof ${ }^{a}$. Dra . Daniela Mariz Silva Vieira - IME-USP 
À minha amada mamãe 



\section{Agradecimentos}

Nesse momento especial é necessário agradecer às pessoas que, ao longo desses anos, contribuíram para que ele ocorrese, especialmente na elaboração e concepção desta tese que coroa o fim desse importante período acadêmico e cujo primeiro artigo originado da mesma foi aceito recentemente para publicação. Meus sinceros agradecimentos:

Ao professor Geraldo Botelho, pela sua fundamental importância durante todas as etapas da construção da tese, desde a proposta do tema, passando pela constante discussão de cada um dos resultados apresentados e encerrando com uma minuciosa revisão e aprimoramento do texto antes de sua submissão à banca.

À professora Mary Lilian, pelo total apoio durante todo curso, especialmente pela proposta e incentivo a minha ida à UFU.

Aos professores Daniel Pellegrino, Vinícius Fávaro, Jorge Mujica e Daniela Vieira, por aceitarem participar da banca examinadora, pela leitura atenta do texto e pelas sugestões precisas, relevantes e que adicionaram significativamente à qualidade do texto.

Aos professores do IME-USP e da FAMAT-UFU, pelo conhecimento transmitido.

À CAPES, pelo apoio financeiro nos primeiros anos.

Ao sr. Zarzur e a sua filha Cláudia, pela acolhida em sua casa em São Paulo e pela companhia e amizade durante o tempo que lá passei.

Aos amigos do IME, da FAMAT, dos pensionatos de São Paulo e Uberlândia e os de longa data, por todos os bons momentos e apoio (dentro do possível) durante esses quatro anos de curso.

À minha mãe Vera, por ter sido meu principal apoio durante todo os momentos difíceis (que não foram poucos) ao longo desses anos. Sem ela não sei se teria encontrado forças para superá-los. 



\section{Resumo}

TORRES, E. R. Hiper-Ideais de Aplicações Multilineares e Polinômios Homogêneos em Espaços de Banach. 2015. 119 f. Tese (Doutorado) - Instituto de Matemática e Estatística, Universidade de São Paulo, São Paulo, 2015.

Nesse trabalho introduzimos e desenvolvemos a teoria de hiper-ideais de aplicações multilineares contínuas e polinômios homogêneos contínuos entre espaços de Banach. A ideia central é refinar os conceitos de multi-ideais e de ideais de polinômios com o objetivo de explorar de forma mais aprofundada a natureza não-linear das aplicações envolvidas. Para isso tomamos a teoria de ideais de operadores lineares, aplicações multilineares e polinômios homogêneos, desenvolvida a partir dos trabalhos de Pietsch, tanto no caso linear como no caso multilinear, como referencial. Provamos resultados gerais para hiperideais, damos muitos exemplos ilustrativos, e desenvolvemos métodos para gerar hiperideais, tanto no caso multilinear como no caso polinomial.

Palavras-chave: espaços de Banach, aplicações multilineares, polinômios homogêneos, multi-ideais, hiper-ideais. 



\section{Abstract}

TORRES, E. R. Hyper-Ideals of Multilinear Mappings and Homogeneous Polynomials in Banach Spaces. 2015. 119 f. Tese (Doutorado) - Instituto de Matemática e Estatística, Universidade de São Paulo, São Paulo, 2015.

In this work we introduce and develop the theory of hyper-ideals of multilinear mappings and homogeneous polynomials between Banach spaces. The main idea is to refine the concepts of multi-ideal and of ideal of polynomials with the purpose of exploring deeply the nonlinear nature of the underlying mappings. To do this we take the ideal theory of linear operators, multilinear mappings and homogeneous polynomials, developed from the works of Pietsch, both in the linear and nonlinear cases, as a reference. We prove general results for hyper-ideals, provide a number of illustrative examples, and develop methods to generate hyper-ideals of multilinear mappings, as well as of hyper-ideals of homogeneous polynomials.

Keywords: Banach spaces, multilinear mappings, homogeneous polynomials, multiideals, hyper-ideals. 



\section{Lista de Símbolos}

$\begin{array}{ll}\mathbb{N} & \{1,2, \ldots\} \\ \mathbb{R} & \text { conjunto dos números reais } \\ \mathbb{C} & \text { conjunto dos números complexos } \\ \mathbb{K} & \mathbb{R} \text { ou } \mathbb{C} \\ X, Y \text { e } Z & \text { espaços vetoriais ou espaços vetoriais normados sobre o } \\ & \text { corpo } \mathbb{K} \\ E, F, G \text { e } H & \text { espaços de Banach sobre o corpo } \mathbb{K} \\ \ell_{p}(E) & \text { espaço das sequências absolutamente } p \text {-somáveis que to- } \\ & \text { mam seus valores em } E \\ \ell_{p}^{w}(E) & \text { espaço das sequências fracamente } p \text {-somáveis que tomam } \\ \ell_{\infty}(E) & \text { seus valores em } E \\ R a d(E) & \text { espaço das sequências limitadas de } E \\ & \text { conjunto das sequências de Rademacher no espaço de } \\ B_{X} & \text { Banach } E \\ & \text { bola fechada do espaço normado } X \text { com centro na ori- } \\ X^{*} & \text { gem e raio } 1 \\ X^{\prime} & \text { dual algébrico do espaço vetorial } X \\ I d_{E} & \text { dual topológico do espaço normado } X \\ \iota_{j} & \text { operador identidade definido em } E \\ \pi_{j} & \text { inclusão canônica de } E_{j} \text { em } E_{1} \times \cdots \times E_{n}, j=1, \ldots, n \\ I_{n} & \text { projeção canônica de } E_{1} \times \cdots \times E_{n} \text { em } E_{j}, j=1, \ldots, n \\ & \text { forma } n \text {-linear contínua de } \mathbb{K}^{n} \text { em } \mathbb{K} \text { dada por } \\ I_{n}\left(\lambda_{1}, \ldots, \lambda_{n}\right)=\lambda_{1} \cdots \lambda_{n} & \text { aplicações lineares } \\ T, U & \text { funcionais lineares } \\ & \text { formas multicaços multilineares } \\ & \end{array}$




\begin{tabular}{|c|c|}
\hline$P, Q, R, S$ & polinômios homogêneos \\
\hline$\hat{A}$ & $\begin{array}{l}\text { polinômio homogêneo associado a aplicação multilinear } \\
A \text {, definido por } \hat{A}(x)=A(x, \ldots, x)\end{array}$ \\
\hline$\check{P}$ & $\begin{array}{l}\text { aplicação multilinear simétrica associada ao polinômio } \\
\text { homogêneo } P\end{array}$ \\
\hline$A_{\sigma}$ & $\begin{array}{l}\text { aplicação obtida de } A \in \mathcal{L}\left({ }^{n} E ; F\right) \text { dada por } \\
A_{\sigma}\left(x_{1}, \ldots, x_{n}\right)=A\left(x_{\sigma(1)}, \ldots, x_{\sigma(n)}\right) \text {, onde } \sigma \text { é uma per- } \\
\text { mutação de }\{1, \ldots, n\}\end{array}$ \\
\hline$A_{s}$ & $\begin{array}{l}\text { aplicação obtida de } A \in \mathcal{L}\left({ }^{n} E ; F\right) \text { dada por } \\
A_{s}\left(x_{1}, \ldots, x_{n}\right)=\frac{1}{n !} \sum_{\sigma \in S_{n}} A\left(x_{\sigma(1)}, \ldots, x_{\sigma(n)}\right), \text { onde } S_{n} \text { é }\end{array}$ \\
\hline$T_{1} \otimes \cdots \otimes T_{n} \otimes y$ & $\begin{array}{l}\text { o conjunto de todas as permutações de }\{1, \ldots, n\} \\
\text { aplicação } \quad \text { multilinear } \quad \text { (contínua) dada }\end{array}$ \\
\hline & por $\quad T_{1} \otimes \cdots \otimes T_{n} \otimes y\left(x_{1}, \ldots, x_{m_{n}}\right)=$ \\
\hline & $\begin{array}{l}T_{1}\left(x_{1}, \ldots, x_{m_{1}}\right) \cdots T_{n}\left(x_{m_{n-1}+1}, \ldots, x_{m_{n}}\right) \cdot y \text {, onde } T_{j} \text { são } \\
\text { formas multilineares, } y \in F \text { e } m_{1}<m_{2}<\ldots<m_{n}\end{array}$ \\
\hline$P_{1} \otimes \cdots \otimes P_{n} \otimes y$ & $\begin{array}{l}\text { polinômio homogêneo dada por } P_{1} \otimes \cdots \otimes P_{n} \otimes y(x)= \\
P_{1}(x) \cdots P_{n}(x) \text {, onde } P_{j} \text { são polinômios homogêneos que } \\
\text { assumem valores escalares e } y \in F\end{array}$ \\
\hline$L\left(E_{1}, \ldots, E_{n} ; F\right)$ & $\begin{array}{l}\text { espaço vetorial sobre } \mathbb{K} \text { das aplicações multilineares de } \\
E_{1} \times \cdots \times E_{n} \text { em } F\end{array}$ \\
\hline $\mathcal{L}\left(E_{1}, \ldots, E_{n} ; F\right)$ & $\begin{array}{l}\text { espaço vetorial sobre } \mathbb{K} \text { das aplicações multilineares } \\
\text { contínuas de } E_{1} \times \cdots \times E_{n} \text { em } F\end{array}$ \\
\hline$L\left({ }^{n} E ; F\right)$ & $L(E, . \stackrel{(n)}{.}, E ; F)$ \\
\hline $\mathcal{L}\left({ }^{n} E ; F\right)$ & $\mathcal{L}(E, . \stackrel{(n)}{.}, E ; F)$ \\
\hline$L^{s}\left({ }^{n} E ; F\right)$ & $\begin{array}{l}\text { subespaço vetorial de } L\left({ }^{n} E ; F\right) \text { das aplicações multiline- } \\
\text { ares simétricas }\end{array}$ \\
\hline $\mathcal{L}^{s}\left({ }^{n} E ; F\right)$ & $\begin{array}{l}\text { subespaço vetorial de } \mathcal{L}\left({ }^{n} E ; F\right) \text { das aplicações multili- } \\
\text { neares simétricas contínuas }\end{array}$ \\
\hline$P\left({ }^{n} E ; F\right)$ & $\begin{array}{l}\text { espaço vetorial sobre } \mathbb{K} \text { dos polinômios } n \text {-homogêneos } \\
\text { de } E \text { em } F\end{array}$ \\
\hline $\mathcal{P}\left({ }^{n} E ; F\right)$ & $\begin{array}{l}\text { espaço vetorial sobre } \mathbb{K} \text { dos polinômios } n \text {-homogêneos } \\
\text { contínuos de } E \text { em } F\end{array}$ \\
\hline $\mathcal{P}$ & $\begin{array}{l}\text { classe de todos os polinômios homogêneos contínuos en- } \\
\text { tre espaços de Banach }\end{array}$ \\
\hline
\end{tabular}




$$
\begin{aligned}
& x_{1} \otimes \cdots \otimes x_{n} \quad \text { tensor elementar definido por } x_{1} \otimes \cdots \otimes x_{n}(A)= \\
& A\left(x_{1}, \ldots, x_{n}\right) \text { para toda aplicação } A \in L\left(E_{1}, \ldots, E_{n}\right) \\
& E_{1} \otimes \cdots \otimes E_{n} \quad \text { produto tensorial dos espaços de Banach } E_{1}, \ldots, E_{n} \text {, de- } \\
& \text { finido como o subespaço de } L\left(E_{1}, \ldots, E_{n}\right)^{*} \text { gerado pelos } \\
& \text { tensores elementares } \\
& \otimes^{n} E \quad E \otimes \stackrel{(n)}{*} \otimes E \\
& \otimes^{n, s} E \quad \text { produto tensorial simétrico de } E \text {, definido como o su- } \\
& \text { bespaço do produto tensorial } \otimes^{n} E \text { gerado pelos tensores } \\
& \text { da forma } x \otimes \stackrel{(n)}{.} \otimes x, x \in E \\
& \pi_{s} \quad \text { norma } s \text {-tensorial projetiva } \\
& \otimes_{\pi_{s}}^{m, s} E \quad \text { espaço normado }\left(\otimes^{m, s} E, \pi_{s}\right) \\
& \widehat{\otimes}_{\pi_{s}}^{m, s} E \quad \text { completamento do espaço normado } \otimes_{\pi_{s}}^{m, s} E \\
& A_{L} \quad \text { linearização da aplicação multilinear } A \\
& \mathcal{X}, \mathcal{Y} \quad \text { funtores } \\
& \Pi, \Pi_{j} \quad \text { partições }\left\{j_{1}^{(1)}, \ldots, j_{k_{1}}^{(1)}\right\} \cup \cdots \cup\left\{j_{1}^{(m)}, \ldots, j_{k_{m}}^{(m)}\right\} \text { do con- } \\
& \text { junto }\{1, \ldots, n\} \\
& \mathcal{I}, \mathcal{I}_{1}, \ldots, \mathcal{I}_{n} \quad \text { ideais de operadores lineares } \\
& \mathcal{I}(E ; F) \quad \mathcal{I} \cap \mathcal{L}(E ; F) \text {, componente de } \mathcal{I} \\
& \mathcal{G} \quad \text { classe de aplicações multilineares contínuas entre } \\
& \text { espaços de Banach tal que } \mathcal{G}^{1}:=\bigcup_{E, F \in B A N} \mathcal{G}(E ; F) \text { é ideal } \\
& \text { de operadores } \\
& \mathcal{G}\left(E_{1}, \ldots, E_{n} ; F\right) \quad \mathcal{G} \cap \mathcal{L}\left(E_{1}, \ldots, E_{n} ; F\right), \text { componente de } \mathcal{G} \\
& \mathcal{M}, \mathcal{M}_{1}, \ldots, \mathcal{M}_{n} \text { ideais de aplicações multilineares ou multi-ideais } \\
& \mathcal{H}, \mathcal{H}_{1}, \ldots, \mathcal{H}_{n} \quad \text { hiper-ideais de aplicações multilineares } \\
& \mathcal{Q}, \mathcal{Q}_{1}, \ldots, \mathcal{Q}_{n} \quad \text { ideais de polinômios homogêneos } \\
& \mathcal{Q}\left({ }^{n} E ; F\right) \quad \mathcal{Q} \cap \mathcal{L}\left({ }^{n} E ; F\right), \text { componente de } \mathcal{Q} \\
& \mathcal{P}_{\mathcal{G}} \quad\{P \in \mathcal{P}: \check{P} \in \mathcal{G}\} \text {, onde } \mathcal{G} \text { é uma classe de aplicações } \\
& \text { multilineares } \\
& \mathcal{P}^{\mathcal{G}} \quad\{P \in \mathcal{P}: \text { existe } A \in \mathcal{G} \text { tal que } \widehat{A}=P\} \text {, onde } \mathcal{G} \text { é uma } \\
& \text { classe de aplicações multilineares } \\
& \overline{\mathcal{G}} \quad \text { fecho da classe de aplicações multilineares }(\mathcal{G},\|\cdot\|) \text {, cujas } \\
& \text { componentes são } \overline{\mathcal{G}\left(E_{1}, \ldots, E_{n} ; F\right)}\|\cdot\|
\end{aligned}
$$




$\begin{array}{ll}\mathcal{F} & \text { ideal dos operadores de posto finito } \\ \mathcal{C} C & \text { ideal dos operadores completamente contínuos } \\ \mathcal{K} & \text { ideal dos operadores compactos } \\ \mathcal{W} & \text { ideal dos operadores fracamente compactos } \\ \mathcal{L}_{f} & \text { classe das aplicações multilineares de tipo finito } \\ \mathcal{L}_{\mathcal{F}} & \text { classe das aplicações multilineares de posto finito } \\ \mathcal{L}_{\mathcal{N}} & \text { classe das aplicações multilineares nucleares } \\ \mathcal{L}_{\mathcal{H N}}(s, r) & \text { classe das aplicações multilineares hiper- }(s, r) \text {-nucleares } \\ \mathcal{L}_{\mathcal{H N}} & \text { classe das aplicações multilineares hiper-nucleares } \\ \mathcal{L}_{\mathcal{K}_{p}} & \text { classe das aplicações multilineares } p \text {-compactas } \\ \mathcal{L}_{\mathcal{K}} & \text { classe das aplicações multilineares compactas } \\ \mathcal{L}_{\mathcal{W}} & \text { classe das aplicações multilineares fracamente compac- } \\ & \text { tas } \\ \mathcal{L}_{f q s, p} & \text { classe das aplicações mutilineares fortemente quase } p \text { - } \\ & \text { somantes } \\ \mathcal{L}_{w s c} & \text { classe das aplicações fracamente sequencialmente con- } \\ & \text { vergentes } \\ \mathcal{L}_{m s c} & \text { classe das aplicações multilinearmente sequencialmente } \\ \mathcal{P}_{f} & \text { convergentes } \\ \mathcal{P}_{\mathcal{F}} & \text { classe dos polinômios homogêneos de tipo finito } \\ \mathcal{P}_{\mathcal{N}} & \text { classe dos polinômios homogêneos de posto finito } \\ \mathcal{P}_{\mathcal{H N}} & \text { classe dos polinômios homogêneos nucleares } \\ \mathcal{P}_{\mathcal{H N}} & \text { classe dos polinômios homogêneos hiper- }(s, r) \text {-nucleares } \\ \mathcal{P}_{\mathcal{K}_{p}} & \text { classe dos polinômios homogêneos hiper-nucleares } \\ \mathcal{P}_{\mathcal{K}} & \text { classe dos polinômios homogêneos } p \text {-compactos } \\ \mathcal{P}_{\mathcal{W}} & \text { classe dos polinômios homogêneos compactos } \\ \mathcal{P}_{s s}^{p} & \text { classe dos polinômios homogêneos fracamente compac- } \\ & \text { comantes } \\ & \text { clinômios homogêneos fortemente } \\ & \end{array}$




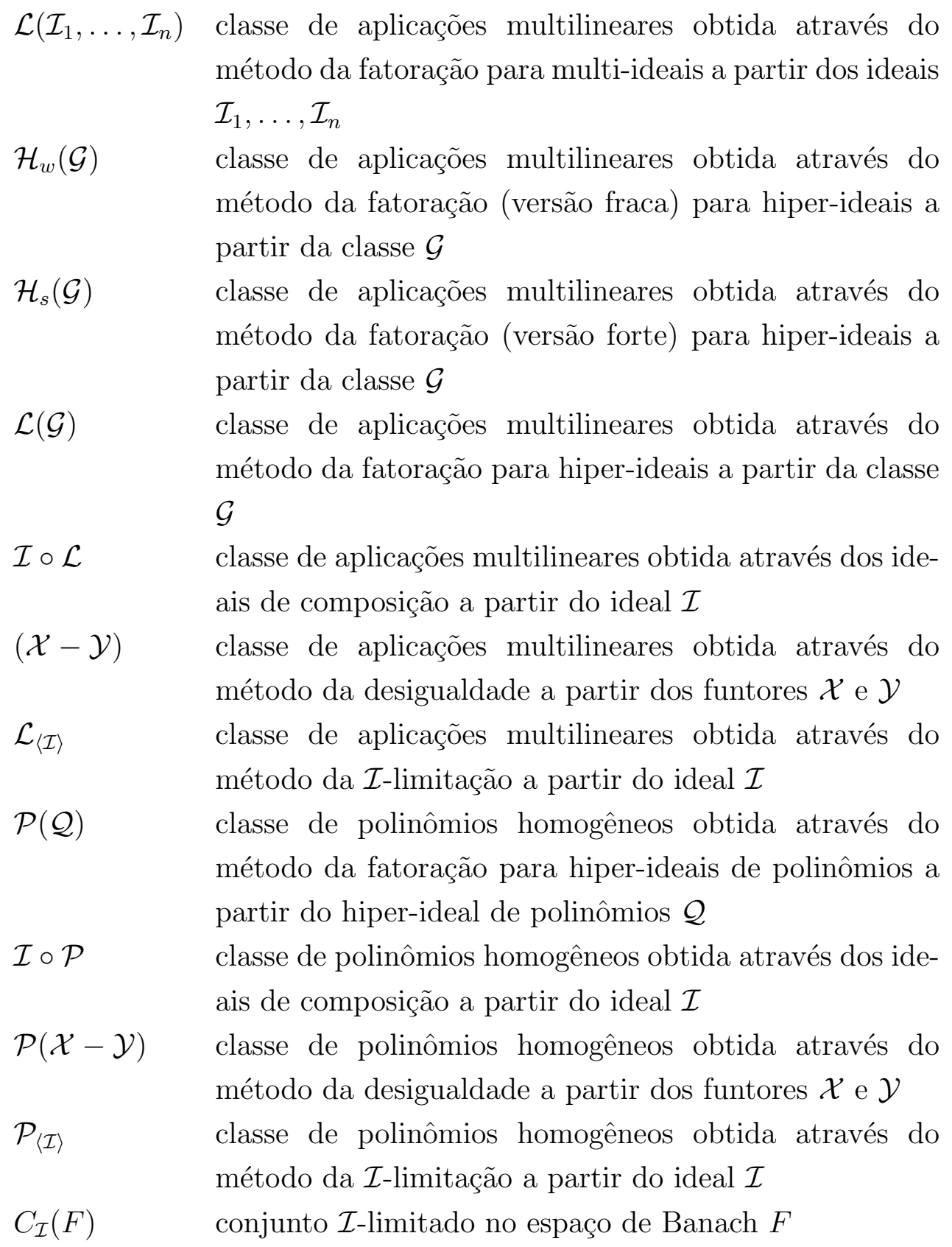





\section{Sumário}

Resumo vii

Abstract $\quad$ ix

Lista de Símbolos $\quad$ xi

Introdução

1 Preliminares $r$

$1.1 p$-norma e espaços de sequências com valores vetoriais . . . . . . . . . . . . 7

1.2 Aplicações multilineares contínuas . . . . . . . . . . . . . . . . . . . . . 9

1.3 Polinômios homogêneos . . . . . . . . . . . . . . . . . . . . . . . 12

1.4 O produto tensorial . . . . . . . . . . . . . . . . 14

1.5 Ideais de aplicações multilineares . . . . . . . . . . . . . . . . . 17

1.6 Ideais de polinômios . . . . . . . . . . . . . . . . . . . . . 21

2 Hiper-ideais de Aplicações Multilineares $\quad 25$

2.1 Definição e propriedades gerais . . . . . . . . . . . . . . 25

2.2 Exemplos . . . . . . . . . . . . . . . . . 36

2.3 Método da fatoração . . . . . . . . . . . . . . . . . . . . . . . . . 48

2.4 Ideais de composição . . . . . . . . . . . . . . . . . . . . . 63

2.5 Método da desigualdade . . . . . . . . . . . . . . . . . . 71

xvii 
2.6 Método da $\mathcal{I}$-limitação . . . . . . . . . . . . . . . . . . . . . . . . . . . 80

3 Hiper-ideais de Polinômios Homogêneos $\quad 89$

3.1 Definição e propriedades básicas . . . . . . . . . . . . . . . . . . . 89

3.2 Exemplos: polinômios de tipo finito, de posto finito e hiper-nucleares . . . 93

3.3 Hiper-ideais de polinômios gerados por hiper-ideais de aplicações multilineares . . . . . . . . . . . . . . . . . . . . . 9 97

3.4 Outros métodos para gerar hiper-ideais de polinômios . . . . . . . . . . . . 102

3.4 .1 Método da fatoração . . . . . . . . . . . . . . . . . . . . . . 102

3.4 .2 Ideais de composição . . . . . . . . . . . . . . . . . 105

3.4 .3 Método da desigualdade . . . . . . . . . . . . . . 107

3.4.4 Método da $\mathcal{I}$-limitação . . . . . . . . . . . . . . . . . . . . 111 


\section{Introdução}

A teoria de ideais de operadores lineares entre espaços de Banach é uma área estabelecida e de muito sucesso dentro da Teoria dos Espaços de Banach. Classes especiais de operadores lineares têm sido consideradas desde os primórdios da Análise Funcional, por exemplo, Hilbert e Riesz já estudavam os operadores que hoje chamamos de compactos. A partir da década de 1960, A. Pietsch empreendeu um trabalho de sistematização das classes de operadores lineares que vinham sendo estudadas individualmente, muitas delas provenientes dos trabalhos de A. Grothendieck, por exemplo, operadores nucleares e absolutamente somantes. Seu trabalho foi coroado com o livro [43], primeiramente publicado em 1978, no qual apresenta o conceito abstrato de ideal de operadores, desenvolve a teoria geral, estuda os principais casos particulares e apresenta muitas aplicações. Desde então ideal de operadores é um tópico muito popular em Análise Funcional e muita pesquisa tem sido feita até os nossos dias. Destacamos também o livro [21] de A. Defant e K. Floret, e o survey [24] de J. Diestel, H. Jarchow e A. Pietsch.

Uma das consequências naturais do sucesso da teoria de ideais de operadores é a extensão da teoria para operadores não-lineares. Um primeiro passo nessa direção foi tomado pelo próprio A. Pietsch em 1983, que no artigo [44] esboça uma teoria de ideais de operadores multilineares, também chamados de multi-ideais. Mais que um artigo de pesquisa, este paper de Pietsch provou ser um programa de pesquisa, pois a partir dele uma quantidade impressionante de trabalhos têm sido feitos na direção por ele apontada; sendo a presente tese uma contribuição a mais nesta fronteira aberta por Pietsch em seu artigo seminal.

A teoria de multi-ideais tem se desenvolvido, principalmente, segundo as seguintes direções:

(i) Estudo das propriedades conhecidas dos ideais de operadores lineares que se estendem ao caso multilinear;

(ii) Criação e desenvolvimento de métodos para gerar multi-ideais, partindo ou não de ideais de operadores lineares; 
(iii) Explorar as propriedades intrinsecamente não-lineares dos multi-ideais conhecidos, bem como dos métodos estabelecidos no item (ii);

(iv) Estender a teoria para outros contextos não-lineares, principalmente para polinômios homogêneos;

$(v)$ Relacionar a teoria de multi-ideais com outros aspectos da análise não-linear e obtenção de aplicações em outros domínios.

Para cada um dos itens acima poderíamos citar um grande número de referências, mas, por brevidade, citamos apenas duas aplicações da teoria de multi-ideais em domínios aparentemente não relacionados: domínio maximal de convergência de séries de Dirichlet vetoriais [22] e teoria da informação quântica [37, 42].

Esta tese pretende ser uma contribuição na direção, principalmente, do item (iii), mas também analisaremos questões relacionadas aos itens $(i)$, (ii) e $(i v)$. Para detalhar a motivação principal desta tese, relembremos a propriedade definidora dos multi-ideais, que diz respeito à estabilidade da classe por composições com operadores lineares. Mais precisamente, se $\mathcal{M}$ é um multi-ideal, então $\mathcal{M}$ é uma subclasse da classe das aplicações multilineares contínuas entre espaços de Banach que satisfaz a seguinte condição: se $G_{1}, \ldots, G_{n}, E_{1}, \ldots, E_{n}, F$ e $H$ são espaços de Banach, $u_{j}: G_{j} \longrightarrow E_{j}, j=1, \ldots, n$, e $t: F \longrightarrow H$ são operadores lineares contínuos, e $A: E_{1} \times \cdots \times E_{n} \longrightarrow F$ é uma aplicação $n$-linear contínua que pertence a $\mathcal{M}$, esquematicamente:

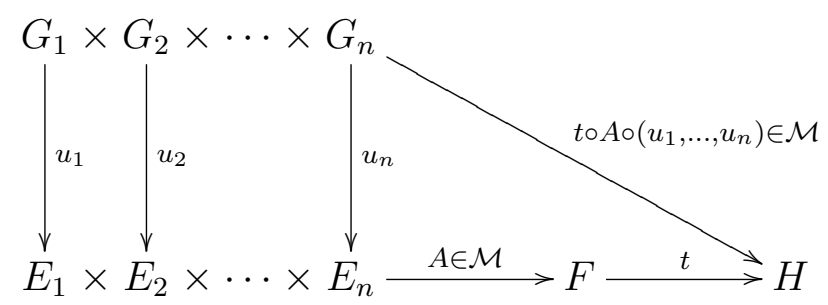

então a composição $t \circ A \circ\left(u_{1}, \ldots, u_{n}\right)$ também pertence a $\mathcal{M}$. A proposta desta tese está baseada na seguinte observação: a propriedade de multi-ideal acima descrita, ao restringir a composição com operadores lineares à esquerda, não explora, em sua totalidade, o caráter multilinear da situação. É claro que à direita só faz sentido compor com operador linear, mas à esquerda faz sentido compor com aplicações multilineares, de graus de multilinearidade arbitrários. Estamos então interessados em classes de aplicações multilineares que são estáveis pela composição com operadores lineares à direita e pela composição com aplicações multilineares à esquerda, classes estas que chamaremos de hiper-ideais. Mais precisamente, estamos interessados em desenvolver a teoria das subclasses $\mathcal{H}$ da classe das aplicações multilineares contínuas entre espaços de Banach que satisfazem a:

Propriedade de hiper-ideal: Se $n$ e $1 \leq m_{1}<\cdots<m_{n}$ são números naturais, 
$G_{1}, \ldots, G_{m_{n}}, E_{1}, \ldots, E_{n}, F$ e $H$ são espaços de Banach, $B_{1} \in \mathcal{L}\left(G_{1}, \ldots, G_{m_{1}} ; E_{1}\right), \ldots, B_{n} \in$ $\mathcal{L}\left(G_{m_{n-1}+1}, \ldots, G_{m_{n}} ; E_{n}\right)$, são aplicações multilineares contínuas, $A: E_{1} \times \cdots \times E_{n} \longrightarrow F$ é uma aplicação multilinear pertencente à classe $\mathcal{H}$, e $t: F \longrightarrow H$ é um operador linear contínuo, esquematicamente:

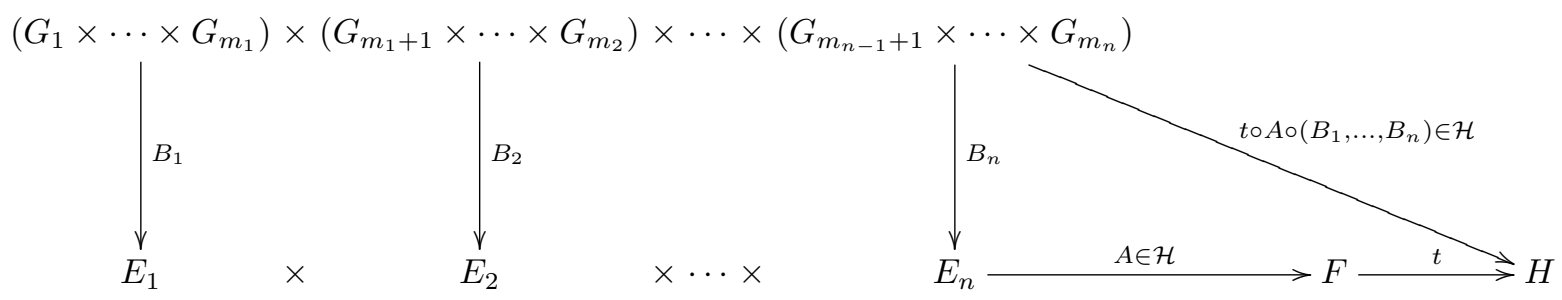

então a composição $t \circ A \circ\left(B_{1}, \ldots, B_{n}\right)$ também pertence à classe $\mathcal{H}$.

Acreditamos que, dessa forma, estamos explorando de forma mais aprofundada a natureza multilinear da situação. A condição de hiper-ideal já foi estudada em alguns poucos casos particulares, por exemplo em $[23,47]$. O objetivo desta tese é introduzir e desenvolver de forma sistemática a teoria de hiper-ideais de aplicações multilineares entre espaços de Banach. Explicaremos a forma na qual isso será feito descrevendo o conteúdo de cada um dos capítulos.

No primeiro capítulo apresentamos os conceitos e resultados básicos e conhecidos que serão utilizados ao longo de todo o trabalho, incluindo as definições precisas de ideais de aplicações multilineares e ideais de polinômios. Incluimos alguns resultados fundamentais e os métodos clássicos de obtenção de multi-ideais a partir de ideais de operadores, além de formas de obter ideais de polinômios através de multi-ideais e de ideais de operadores. Destacamos ainda o conceito de produto tensorial projetivo, que será muito útil ao longo do texto.

No Capítulo 2 começamos a parte original da tese introduzindo a definição de hiperideal de aplicações multilineares e apresentando suas propriedades básicas inspiradas em propriedades de multi-ideais e de ideais de operadores. Em seguida apresentamos vários exemplos e ressaltamos as distinções entre multi-ideais e hiper-ideais. Uma diferença importante que estabelecemos é que, enquanto multi-ideais exigem a presença das aplicações multilineares de tipo finito, hiper-ideais exigem, como consequência da propriedade de hiper-ideal, a presença das aplicações multilineares de posto finito. Assim vários multiideais são descartados da teoria de hiper-ideais.

Ainda neste segundo capítulo nos preocupamos em transpor para hiper-ideais os métodos de obtenção de multi-ideais a partir de ideais de operadores. No primeiro método testado, o da fatoração, a característica mais restritiva dos hiper-ideais se impôs com toda a sua força. As dificuldades impostas pela propriedade de hiper-ideal aparecem no primeiro momento, e buscamos várias alternativas de um método da fatoração satisfatório 
para hiper-ideais completos. Cada uma das alternativas tem seus prós e seus contras, e apresentamos todas elas para deixar bem claros os limites impostos pela propriedade de hiper-ideal. Por outro lado, mostramos que os ideais de composição são totalmente compatíveis com a noção de hiper-ideal. Sem alterar nada do método original para multiideais, esse método gera naturalmente hiper-ideais com todas as propriedades desejadas. Como compensação para os resultados insatisfatórios do método da fatoração, apresentamos dois novos métodos para a geração de hiper-ideais. Os dois se baseiam em técnicas que já vinham sendo aplicadas em casos isolados, o que fazemos é sistematizar o procedimento e mostrar que geram hiper-ideais. O método da desigualdade gera hiper-ideais a partir de uma definição que não requer uma classe inicial e fornece classes conhecidas, por exemplo a classe das aplicações multilineares fortemente somantes estudada em [26], como exemplos de hiper-ideais. Já o método da $\mathcal{I}$-limitação gera um hiper-ideal $\mathcal{L}_{\langle\mathcal{I}\rangle}$ a partir de um ideal de operadores $\mathcal{I}$, e também fornece novos exemplos de hiper-ideais.

Como era de se esperar, a noção de multi-ideal foi rapidamente acompanhada pela noção de ideais de polinômios homogêneos. Não se sabe ao certo quando a noção de ideais de polinômios homogêneos foi formalizada pela primeira vez, mas a relação entre multi-ideais e ideais de polinômios aparece na tese [15] de H.-A.Braunss de 1984. As duas teorias, multi-ideais e ideais de polinômios, têm se desenvolvido de forma paralela, e, de forma muito natural, estudamos no último capítulo desta tese o conceito de hiper-ideais de polinômios homogêneos. A definição é bem natural: relembre que um ideal de polinômios $\mathcal{Q}$ é uma subclasse da classe dos polinômios homogêneos contínuos entre espaços de Banach que satisfaz a seguinte propriedade: se $P: E \longrightarrow F$ é um polinômio $n$-homogêneo que pertence à classe $\mathcal{Q}$ e $u: G \longrightarrow E$ e $t: F \longrightarrow H$ são operadores lineares contínuos, esquematicamente

$$
G \stackrel{u}{\longrightarrow} E \stackrel{P}{\longrightarrow} F \stackrel{t}{\longrightarrow} H
$$

então o polinômio $n$-homogêneo $t \circ P \circ u$ também pertence à classe $\mathcal{Q}$. Mantendo a filosofia de hiper-ideais, estaremos interessados em classes de polinômios $\mathcal{Q}$, os quais chamaremos de hiper-ideais de polinômios, que satisfazem a seguinte condição mais restritiva: se $P: E \longrightarrow F$ é um polinômio $n$-homogêneo que pertence à classe $\mathcal{Q}, Q: G \longrightarrow E$ é um polinômio $m$-homogêneo e $t: F \longrightarrow H$ é um operador linear contínuo, esquematicamente

$$
G \stackrel{Q}{\longrightarrow} E \stackrel{P}{\longrightarrow} F \stackrel{t}{\longrightarrow} H
$$

então o polinômio $n m$-homogêneo $t \circ P \circ Q$ também pertence à classe $\mathcal{Q}$. No terceiro capítulo da tese intoduzimos e desenvolvemos a teoria de hiper-ideais de polinômios, de forma paralela à teoria de multi-ideais. Para realizar esse estudo seguimos o roteiro 
estabelecido no capítulo anterior, passando por todas as propriedades básicas de hiperideais de polinômios e adaptando os métodos para gerar hiper-ideais de polinômios. A especificidade deste capítulo é a seção que ensina como obter hiper-ideais de polinômios a partir de hiper-ideais de aplicações multilineares. Para isso foi necessário introduzir a noção de hiper-ideal simétrico. Procedendo desta forma, para cada método de gerar hiperideais de polinômios podemos tanto adaptar para polinômios o método correspondente para hiper-ideais, como considerar o hiper-ideal de polinômios gerado pelo hiper-ideal correspondente. Para cada método fazemos um estudo comparativo entre essas duas possibilidades de se gerar hiper-ideais de polinômios. 


\section{Capítulo 1}

\section{Preliminares}

Neste capítulo introduzimos os conceitos básicos, e suas respectivas notações, que serão necessários para o desenvolvimento da tese. O objetivo central da tese é introduzir e desenvolver a teoria de uma noção que refina os conceitos de ideais de aplicações multilineares e de ideais de polinômios homogêneos. Para isso compilamos neste capítulo as definições, notações e resultados acerca destes conceitos que necessitaremos no desenvolvimento do trabalho.

Dado um espaço normado $E$, por $E^{\prime}$ denotamos seu dual topológico munido da norma uniforme de funcionais lineares e por $B_{E}$ sua bola unitária fechada. Ao longo do trabalho, todos os espaços vetoriais são sobre o corpo $\mathbb{K}=\mathbb{R}$ ou $\mathbb{C}$.

\section{$1.1 p$-norma e espaços de sequências com valores ve- toriais}

Espaços p-normados, e não apenas espaços normados, desempenham papel relevante na teoria de ideais, mesmo no caso linear. Por isso fazemos aqui uma pequena introdução aos espaços $p$-normados e aos espaços $p$-normados de sequências a valores vetoriais.

Definição 1.1.1. Sejam $X$ um espaço vetorial e $0<p \leq 1$. Uma função $\|\cdot\|: X \longrightarrow$ $[0, \infty)$ é uma $p$-norma em $X$ se satisfaz as seguintes condições:

(i) $\|x\|=0$ se, e somente se, $x=0$;

(ii) $\|\lambda x\|=|\lambda| \cdot\|x\|$ para todo $x \in X$ e $\lambda \in \mathbb{K}$;

(iii) $\|x+y\|^{p} \leq\|x\|^{p}+\|y\|^{p}$ para quaisquer $x, y \in X$. 
Quando munimos $X$ com essa função dizemos que o par $(X,\|\cdot\|)$ é um espaço vetorial p-normado, ou simplesmente um espaço p-normado. Neste caso, a função

$$
(x, y) \in X \times X \longmapsto\|x-y\|^{p} \in \mathbb{R}
$$

é uma métrica em $X$, e consideraremos em $X$ a estrutura topológica de espaço métrico induzida por esta métrica. Se $X$ for completo em relação a esta métrica, dizemos que $(X,\|\cdot\|)$ é um espaço $p$-Banach.

Quando $p=1$ dizemos simplesmente que $X$ é normado e Banach, respectivamente.

Para mais detalhes sobre espaços $p$-normados e espaços $p$-Banach, nos referimos ao survey de Kalton [34].

Dentre os espaços $p$-Banach que usaremos ao longo do trabalho, convém introduzir certos espaços de sequências que desempenharão um papel importante em seções futuras. Aqui estaremos considerando $(E,\|\cdot\|)$ um espaço de Banach e $p>0$.

- Sequências absolutamente somáveis. Por $\ell_{p}(E)$ denotamos o espaço das sequências $\left(x_{j}\right)_{j=1}^{\infty}$ em $E$ tais que $\sum_{j=1}^{\infty}\left\|x_{j}\right\|^{p}<\infty$. A expressão

$$
\left\|\left(x_{j}\right)_{j=1}^{\infty}\right\|_{p}=\left(\sum_{j=1}^{\infty}\left\|x_{j}\right\|^{p}\right)^{\frac{1}{p}}
$$

define uma $p$-norma em $\ell_{p}(E)$ se $0<p<1$ e uma norma se $p \geq 1$. Com essa $p$-norma (norma), $\ell_{p}(E)$ é um espaço $p$-Banach (Banach). Seguindo a praxe, quando $E=\mathbb{K}$ escrevemos simplesmente $\ell_{p}$ no lugar de $\ell_{p}(\mathbb{K})$.

- Sequências fracamente somáveis. Por $\ell_{p}^{w}(E)$ denotamos o espaço das sequências $\left(x_{j}\right)_{j=1}^{\infty}$ em $E$ tais que $\sum_{j=1}^{\infty}\left|\varphi\left(x_{j}\right)\right|^{p}<\infty$ para todo funcional $\varphi \in E^{\prime}$. A expressão

$$
\left\|\left(x_{j}\right)_{j=1}^{\infty}\right\|_{w, p}=\sup _{\varphi \in B_{E^{\prime}}}\left(\sum_{j=1}^{\infty}\left|\varphi\left(x_{j}\right)\right|^{p}\right)^{\frac{1}{p}}
$$

define uma $p$-norma em $\ell_{p}^{w}(E)$ se $0<p<1$ e uma norma se $p \geq 1$. Com essa $p$-norma (norma), $\ell_{p}^{w}(E)$ é um espaço $p$-Banach (Banach). É imediato que $\ell_{p}^{w}(\mathbb{K})=\ell_{p}$.

- Sequências limitadas Por $\ell_{\infty}(E)$ denotamos o espaço das sequências limitadas de E. A expressão

$$
\left\|\left(x_{j}\right)_{j=1}^{\infty}\right\|_{\infty}=\sup _{j \in \mathbb{N}}\left\|x_{j}\right\|
$$


é uma norma em $\ell_{\infty}(E)$, que assim se torna um espaço de Banach. Poderíamos definir $\ell_{\infty}^{w}(E)$ analogamente a $\ell_{p}^{w}(E)$, mas nesse caso obteríamos $\ell_{\infty}^{w}(E)=\ell_{\infty}(E)$. Para todo $0<p \leq \infty$ e todo espaço de Banach $E$, é verdade que

$$
\ell_{p}(E) \subseteq \ell_{p}^{w}(E) \subseteq \ell_{\infty}(E)
$$

Mais ainda, $\ell_{p}(E)=\ell_{p}^{w}(E)$ se, e somente se, $E$ tem dimensão finita.

As demonstrações de todas as afirmações feitas acima podem ser encontradas no Capítulo 2 do livro [25].

\subsection{Aplicações multilineares contínuas}

Apresentamos nesta seção os conceitos e resultados básicos sobre aplicações multilineares contínuas entre espaços de Banach.

Definição 1.2.1. Sejam $X_{1}, \ldots, X_{n}$ e $Y$ espaços vetoriais. Uma aplicação $A: X_{1} \times \ldots \times$ $X_{n} \longrightarrow Y$ é dita multilinear (ou $n$-linear), se é linear em cada uma das suas coordenadas, isto é, se

$$
A\left(x_{1}, \ldots, x_{l}+\lambda y_{l}, \ldots, x_{n}\right)=A\left(x_{1}, \ldots, x_{l}, \ldots, x_{n}\right)+\lambda A\left(x_{1}, \ldots, y_{l}, \ldots, x_{n}\right),
$$

para todos $x_{l}, y_{l} \in X_{l}, \lambda \in \mathbb{K}$ e $l=1, \ldots, n$. O conjunto de tais aplicações é denotado por $L\left(X_{1}, \ldots, X_{n} ; Y\right)$, que obviamente se torna um espaço vetorial com as operações pontuais de funções.

Se $X_{1}, \ldots, X_{n}$ e $Y$ forem espaços normados, denotaremos por $\mathcal{L}\left(X_{1}, \ldots, X_{n} ; Y\right)$ o subespaço vetorial de $L\left(X_{1}, \ldots, X_{n} ; Y\right)$ formado pelas aplicações $n$-lineares contínuas. No caso em que $X_{1}=\cdots=X_{n}=X$, escrevemos $L\left({ }^{n} X ; Y\right)$ e $\mathcal{L}\left({ }^{n} X ; Y\right)$ respectivamente. E no caso em que $Y=\mathbb{K}$ escrevemos simplesmente $L\left(X_{1}, \ldots, X_{n}\right), \mathcal{L}\left(X_{1}, \ldots, X_{n}\right), L\left({ }^{n} X\right)$ e $\mathcal{L}\left({ }^{n} X\right)$.

Muito útil será a seguinte caracterização:

Proposição 1.2.2. (Veja [38, Proposition 1.2]) As seguintes afirmações são equivalentes para uma aplicação n-linear $A \in L\left(X_{1}, \ldots, X_{n} ; Y\right)$ :

(a) $A \in \mathcal{L}\left(X_{1}, \ldots, X_{n} ; Y\right)$;

(b) A é contínua na origem; 
(c) Existe uma constante $C>0$ tal que $\left\|A\left(x_{1}, \ldots, x_{n}\right)\right\| \leq C\left\|x_{1}\right\| \cdots\left\|x_{n}\right\|$ para quaisquer $x_{l} \in X_{l}, l=1, \ldots, n$;

$(d) \sup \left\{\left\|A\left(x_{1}, \ldots, x_{n}\right)\right\| ; x_{l} \in B_{X_{l}}\right.$ para todo $\left.l=1 \ldots, n\right\}<\infty$.

Os itens $(c)$ e $(d)$ sugerem uma forma de definir uma norma em $\mathcal{L}\left(X_{1}, \ldots, X_{n} ; Y\right)$. A proposição a seguir nos informa não apenas que isso é possível, como fornece resultados adicionais.

Proposição 1.2.3. (Veja [38, Proposition 1.3]) Para cada $A \in \mathcal{L}\left(X_{1}, \ldots, X_{n} ; Y\right)$, defina

$$
\|A\|:=\sup \left\{\left\|A\left(x_{1}, \ldots, x_{n}\right)\right\| ; x_{l} \in X_{l} \text { e }\left\|x_{l}\right\| \leq 1 \text { para todo } l=1 \ldots, n\right\} .
$$

Então:

(i) $\|\cdot\|$ é uma norma em $\mathcal{L}\left(X_{1}, \ldots, X_{n} ; Y\right)$;

(ii) $\left\|A\left(x_{1}, \ldots, x_{n}\right)\right\| \leq\|A\| \cdot\left\|x_{1}\right\| \cdots\left\|x_{n}\right\|$ para quaisquer $x_{l} \in X_{l}$, com $l=1, \ldots, n$;

(iii) $\|A\|=\inf \left\{C:\left\|A\left(x_{1}, \ldots, x_{n}\right)\right\| \leq C\left\|x_{1}\right\| \cdots\left\|x_{n}\right\|\right.$ com $\left.x_{1} \in X_{1}, \ldots, x_{n} \in X_{n}\right\}$.

Além disso, se $F$ é um espaço de Banach, então $\left(\mathcal{L}\left(X_{1}, \ldots, X_{n} ; F\right),\|\cdot\|\right)$ também é um espaço de Banach.

A norma da Proposição 1.2.3 será referida como norma uniforme ou norma do sup e, salvo menção em contrário, estará munindo o espaço $\mathcal{L}\left(X_{1}, \ldots, X_{n} ; F\right)$ e seus subespaços.

Estaremos sempre utilizando $E_{1}, \ldots, E_{n}$ e $F$ para indicarmos espaços de Banach. Frequentemente trabalharemos com aplicações multilineares contínuas no lugar de aplicações multilineares em geral, mas isso não significa que os resultados subsequentes não tenham seus análogos no caso geral. Trata-se apenas de uma escolha visando a fluidez do texto e a ultilização destes resultados em capítulos posteriores.

Destacamos a seguir duas classes de aplicações multilineares contínuas que serão importantes para a definição de multi-ideais (Definição 1.5.2) e de hiper-ideais (Definição 2.1.2).

Exemplo 1.2.4. Dizemos que uma aplicação multilinear $A \in \mathcal{L}\left(E_{1}, \ldots, E_{n} ; F\right)$ é de tipo finito se existem $k \in \mathbb{N}$, funcionais lineares $\varphi_{j}^{(l)} \in E_{l}^{\prime}$ e vetores $y_{j} \in F, \operatorname{com} j=1, \ldots, k$ e $l=1, \ldots, n$, tais que

$$
A\left(x_{1}, \ldots, x_{n}\right)=\sum_{j=1}^{k} \varphi_{j}^{(1)}\left(x_{1}\right) \cdots \varphi_{j}^{(n)}\left(x_{n}\right) y_{j}
$$


para todo $\left(x_{1}, \ldots, x_{n}\right) \in E_{1} \times \cdots \times E_{n}$. Esta aplicação $n$-linear $A$ também é denotada por $\sum_{j=1}^{k} \varphi_{j}^{(1)} \otimes \cdots \otimes \varphi_{j}^{(n)} \otimes y_{j}$. O subespaço vetorial de $\mathcal{L}\left(E_{1}, \ldots, E_{n} ; F\right)$ formado pelas aplicações de tipo finito será denotado por $\mathcal{L}_{f}\left(E_{1}, \ldots, E_{n} ; F\right)$.

Exemplo 1.2.5. Dizemos que uma aplicação multilinear $A \in \mathcal{L}\left(E_{1}, \ldots, E_{n} ; F\right)$ é de posto finito se a dimensão do subespaço vetorial de $F$ gerado pela imagem de $A$ tem dimensão finita, ou, equivalentemente (veja, por exemplo, [45, Proposição 4.2.1]), se existem $k \in \mathbb{N}$, formas $n$-lineares $T_{j} \in \mathcal{L}\left(E_{1}, \ldots, E_{n}\right)$ e vetores $y_{j} \in F$, para $j=1, \ldots, k$, tais que

$$
A\left(x_{1}, \ldots, x_{n}\right)=\sum_{j=1}^{k} T_{j}\left(x_{1}, \ldots, x_{n}\right) y_{n},
$$

para todo $\left(x_{1}, \ldots, x_{n}\right) \in E_{1} \times \cdots \times E_{n}$. Esta aplicação $n$-linear $A$ também é denotada por $\sum_{j=1}^{k} T_{j} \otimes y_{j}$. O subespaço vetorial de $\mathcal{L}\left(E_{1}, \ldots, E_{n} ; F\right)$ formado pelas aplicações de posto finito será denotado por $\mathcal{L}_{\mathcal{F}}\left(E_{1}, \ldots, E_{n} ; F\right)$. É claro que

$$
\mathcal{L}_{f}\left(E_{1}, \ldots, E_{n} ; F\right) \subseteq \mathcal{L}_{\mathcal{F}}\left(E_{1}, \ldots, E_{n} ; F\right)
$$

No caso em que pelo menos um dos espaços do domínio tem dimensão infinita, nenhum desses subespaços é fechado em $\mathcal{L}\left(E_{1}, \ldots, E_{n} ; F\right)$.

Proposição 1.2.6. (Veja [38, Proposition 1.4]) Sejam $E_{1}, \ldots, E_{n}$ e F espaços de Banach. Dado $m \in \mathbb{N}$ com $m<n$, existe um isomorfismo isométrico canônico

$$
I: \mathcal{L}\left(E_{1}, \ldots, E_{n} ; F\right) \longrightarrow \mathcal{L}\left(E_{1}, \ldots, E_{m} ; \mathcal{L}\left(E_{m+1}, \ldots, E_{n} ; F\right)\right)
$$

definido por

$$
I(A)\left(x_{1}, \ldots, x_{m}\right)\left(x_{m+1}, \ldots, x_{n}\right)=A\left(x_{1}, \ldots, x_{n}\right) .
$$

Para preparar a próxima seção, na qual estudaremos polinômios homogêneos, apresentamos a definição de aplicação multilinear simétrica e a fórmula de polarização. Por $S_{n}$ denotamos o conjunto de todas as bijeções do conjunto $\{1, \ldots, n\}$ sobre si mesmo (permutações dos primeiros $n$ números naturais).

Definição 1.2.7. Uma aplicação multilinear $A \in L\left({ }^{n} E ; F\right)$ é dita simétrica se

$$
A\left(x_{1}, \ldots, x_{n}\right)=A\left(x_{\sigma(1)}, \ldots, x_{\sigma(n)}\right),
$$

para quaisquer $x_{1}, \ldots, x_{n} \in E$, e toda bijeção $\sigma \in S_{n}$. O subespaço vetorial de $L\left({ }^{n} E ; F\right)$ formado pelas aplicações $n$-lineares simétricas será denotado por $L^{s}\left({ }^{n} E ; F\right)$. 
Por simplicidade, dados $A \in \mathcal{L}\left({ }^{n} E ; F\right)$ e $x \in E$, escreveremos $A(x)^{n}$ ou $A x^{n}$ no lugar de $A(x, \ldots, x)$.

Proposição 1.2.8 (Fórmula de Polarização). (Veja [38, Theorem 1.10]) Seja $A \in L^{s}\left({ }^{n} E ; F\right)$. Para quaisquer $x_{1}, \ldots, x_{n} \in E$, temos

$$
A\left(x_{1}, \ldots, x_{n}\right)=\frac{1}{n ! 2^{n}} \sum_{\varepsilon_{j}= \pm 1} \varepsilon_{1} \cdots \varepsilon_{n} A\left(\varepsilon_{1} x_{1}+\cdots+\varepsilon_{n} x_{n}\right)^{n} .
$$

\subsection{Polinômios homogêneos}

Os polinômios homogêneos são as funções que permitem estudar funções holomorfas em espaços normados complexos. Isso ficará mais claro após a definição.

Definição 1.3.1. Sejam $E$ e $F$ espaços normados. Uma aplicação $P: E \longrightarrow F$ é chamada de polinômio n-homogêneo se existe $A \in L\left({ }^{n} E ; F\right)$ tal que $P(x)=A x^{n}$ para todo $x \in E$. Neste caso dizemos que $P$ é o polinômio $n$-homogêneo gerado pela aplicação $n$-linear $A$ e representamos este fato pela igualdade $P=\widehat{A}$. O espaço vetorial dos polinômios $n$-homogêneos de $E$ em $F$ é denotado por $P\left({ }^{n} E ; F\right)$, e seu subespaço formado pelos polinômios $n$-homogêneos contínuos por $\mathcal{P}\left({ }^{n} E ; F\right)$.

Observe que, dados $P \in \mathcal{P}\left({ }^{n} E ; F\right), x \in E$ e $\lambda \in \mathbb{K}$,

$$
P(\lambda x)=A(\lambda x, \ldots, \lambda x)=\lambda^{n} A x^{n}=\lambda^{n} P(x),
$$

onde $\widehat{A}=P$. Assim, polinômios $n$-homogêneos satisfazem a propriedade fundamental da função $z \in \mathbb{C} \mapsto z^{n} \in \mathbb{C}$, e permitem portanto a consideração de séries de potências em espaços normados complexos, e, consequentemente, o estudo de funções holomorfas. Maiores detalhes podem ser encontrados em [38].

A continuidade de um polinômio homogêneo é caracterizada pela seguinte proposição. Proposição 1.3.2. (Veja [38, Proposition 2.4]) As seguintes afirmações são equivalentes para um polinômio n-homogêneo $P \in P\left({ }^{n} E ; F\right)$ :

(a) $P \in \mathcal{P}\left({ }^{n} E ; F\right)$;

(b) P é contínuo na origem;

(c) Existe uma constante $C>0$ tal que $\|P(x)\| \leq C\|x\|^{n}$ para todo $x \in E$;

(d) $\sup \{\|P(x)\| ; x \in E$ e $\|x\| \leq 1\}<\infty ;$

(e) Existe $A \in \mathcal{L}\left({ }^{n} E ; F\right)$ tal que $P=\widehat{A}$. 
Uma norma natural em $\mathcal{P}\left({ }^{n} E ; F\right)$ é dada a seguir.

Proposição 1.3.3. (Veja [38, Corollary 2.3]) Para cada polinômio n-homogêneo $P \in$ $\mathcal{P}\left({ }^{n} E ; F\right)$, defina

$$
\|P\|:=\sup \{\|P(x)\| ; x \in E \text { e }\|x\| \leq 1\} .
$$

Então:

(i) $\|\cdot\|$ é uma norma em $\mathcal{P}\left({ }^{n} E ; F\right)$;

(ii) $\|P(x)\| \leq\|P\| \cdot\|x\|^{n}$ para todo $x \in E ;$

(iii) $\|P\|=\inf \left\{C:\|P(x)\| \leq C \cdot\|x\|^{n}\right.$ para todo $\left.x \in E\right\}$.

Além disso, se $F$ é um espaço de Banach, então $\left(\mathcal{P}\left({ }^{n} E ; F\right),\|\cdot\|\right)$ também é um espaço de Banach.

Estabeleceremos agora a relação entre polinômios $n$-homogêneos e aplicações $n$-lineares simétricas, mencionada no final da seção anterior.

Proposição 1.3.4. (Veja [38, Theorem 2.2]) A aplicação que associa uma aplicação nlinear $A \in L^{s}\left({ }^{n} E ; F\right)$ ao seu polinômio associado $\widehat{A} \in P\left({ }^{n} E ; F\right)$ é um isomorfismo de espaços vetoriais entre $L^{s}\left({ }^{n} E ; F\right)$ e $P\left({ }^{n} E ; F\right)$. Além disso, esse isomorfismo induz um isomorfismos topológico entre os espaços normados $\mathcal{L}^{s}\left({ }^{n} E ; F\right)$ e $\mathcal{P}\left({ }^{n} E ; F\right)$ no qual vale

$$
\|\widehat{A}\| \leq\|A\| \leq \frac{n^{n}}{n !}\|\widehat{A}\|
$$

para toda $A \in \mathcal{L}^{s}\left({ }^{n} E ; F\right)$.

Observação 1.3.5. (i) Para cada polinômio $n$-homogêneo $P \in P\left({ }^{n} E ; F\right)\left(\mathcal{P}\left({ }^{n} E ; F\right)\right.$, respectivamente), indicaremos por $\check{P} \in L\left({ }^{n} E ; F\right)\left(\mathcal{L}\left({ }^{n} E ; F\right)\right.$, respectivamente) a única aplicação multilinear (contínua) simétrica associada a $P$.

(ii) As constantes fornecidas em (1.1) são as melhores possíveis, fato este que pode ser verificado em [38, Exercises 2.H e 2.I] ou em [1, Exemplo 1.2.3].

(iii) Com esse último resultado podemos adicionar mais um item às equivalências da Proposição 1.3.2:

(f) A aplicação $n$-linear simétrica $\check{P}$ é contínua.

Exemplo 1.3.6. Usando os mesmos princípios por meio dos quais as aplicações multilineares de tipo finito e de posto finito foram definidas, podemos obter suas versões polinomiais. Dizemos que um polinômio $P \in \mathcal{P}\left({ }^{n} E ; F\right)$ é de tipo finito se podemos escrever

$$
P(x)=\sum_{j=1}^{k} \varphi_{j}(x)^{n} y_{j}
$$


para todo $x \in E$, onde $k \in \mathbb{N}, \varphi_{j} \in E^{\prime}$ e $y_{j} \in F$ para $j=1, \ldots, k$. Este polinômio $P$ também é denotado por $\sum_{j=1}^{k} \varphi_{j}^{n} \otimes y_{j}$. O subespaço de $\mathcal{P}\left({ }^{n} E ; F\right)$ formado pelos polinômios de tipo finito é denotado por $\mathcal{P}_{f}\left({ }^{n} E ; F\right)$.

Agora, se pudemos escrever

$$
P(x)=\sum_{j=1}^{k} Q_{j}(x) y_{j}
$$

para todo $x \in E$, onde $Q_{j} \in \mathcal{P}\left({ }^{n} E\right)$ e $y_{j} \in F$ para $j=1, \ldots, k$, então dizemos que $P$ tem posto finito. Este polinômio $P$ também é denotado por $\sum_{j=1}^{k} Q_{j} \otimes y_{j}$. O subespaço de $\mathcal{P}\left({ }^{n} E ; F\right)$ formado pelos polinômios de posto finito é denotado por $\mathcal{P}_{\mathcal{F}}\left({ }^{n} E ; F\right)$.

Dado $P \in \mathcal{P}\left({ }^{n} E ; F\right)$, é fácil verificar que

$$
\begin{aligned}
& P \in \mathcal{P}_{f}\left({ }^{n} E ; F\right) \Longleftrightarrow \check{P} \in \mathcal{L}_{f}\left({ }^{n} E ; F\right) \text { e } \\
& P \in \mathcal{P}_{\mathcal{F}}\left({ }^{n} E ; F\right) \Longleftrightarrow \check{P} \in \mathcal{L}_{\mathcal{F}}\left({ }^{n} E ; F\right) .
\end{aligned}
$$

\section{$1.4 \mathrm{O}$ produto tensorial}

Nesta seção construiremos, para uso posterior, o produto tensorial de espaços vetoriais e faremos uma breve exposição sobre a linearização de aplicações multilineares.

Sejam $X_{1}, \ldots, X_{n}$ espaços vetoriais. Dados $x_{1} \in X_{1}, \ldots, x_{n} \in X_{n}$, considere o funcional linear

$$
x_{1} \otimes \cdots \otimes x_{n}: L\left(X_{1}, \ldots, X_{n}\right) \longrightarrow \mathbb{K}
$$

definido por

$$
x_{1} \otimes \cdots \otimes x_{n}(T)=T\left(x_{1}, \ldots, x_{n}\right) .
$$

O produto tensorial de $X_{1}, \ldots, X_{n}$ é o subespaço do dual algébrico de $L\left(X_{1}, \ldots, X_{n}\right)$ gerado pelos funcionais da forma

$$
x_{1} \otimes \cdots \otimes x_{n}
$$

Denotamos esse espaço por $X_{1} \otimes \cdots \otimes X_{n}$ e chamamos seus elementos de tensores. Já seus geradores, isto é, os vetores da forma $x_{1} \otimes \cdots \otimes x_{n}$, com $x_{1} \in X_{1}, \ldots, x_{n} \in X_{n}$, são chamados de tensores elementares. Dessa forma, um tensor $x \in X_{1} \otimes \cdots \otimes X_{n}$ é uma combinação linear de tensores elementares, ou seja, é da forma

$$
x=\sum_{j=1}^{k} \lambda_{j} x_{j}^{1} \otimes \cdots \otimes x_{j}^{n},
$$


onde $\lambda_{j} \in \mathbb{K}$ e $x_{j}^{l} \in X_{l}$ para $l=1, \ldots, k$. Como é de se esperar, os vetores elementares carregam propriedades das aplicações multilineares, as quais listamos a seguir.

Proposição 1.4.1. (Veja [51, Proposição 1.6]) Sejam $X_{1}, \ldots, X_{n}$ espaços vetoriais. Para todo $i=1, \ldots, n$ e quaisquer elementos $x_{1} \in X_{1}, \ldots, x_{i}, y_{i} \in X_{i}, \ldots x_{n} \in X_{n}$, valem:

(a) $x_{1} \otimes \cdots \otimes\left(x_{i}+y_{i}\right) \otimes \cdots \otimes x_{n}=x_{1} \otimes \cdots \otimes x_{i} \otimes \cdots \otimes x_{n}+x_{1} \otimes \cdots \otimes y_{i} \otimes \cdots \otimes x_{n}$;

(b) $\lambda\left(x_{1} \otimes \cdots \otimes x_{i} \otimes \cdots \otimes x_{n}\right)=x_{1} \otimes \cdots \otimes\left(\lambda x_{i}\right) \otimes \cdots \otimes x_{n}$;

(c) $x_{1} \otimes \cdots \otimes 0 \otimes \cdots \otimes x_{n}=0$.

De acordo com o item $(b)$ da proposição acima, podemos escrever um tensor arbitrário $x \in X_{1} \otimes \cdots \otimes X_{n}$ simplesmente como

$$
x=\sum_{j=1}^{k} x_{j}^{1} \otimes \cdots \otimes x_{j}^{n},
$$

$\operatorname{com} x_{j}^{l} \in X_{l}, l=1, \ldots, k$.

Uma forma muito útil de determinar se um tensor $x \in X_{1} \otimes \cdots \otimes X_{n}$ é nulo (ou, equivalentemente, que dois tensores são iguais) é dada pela:

Proposição 1.4.2. (Veja [49, Proposition 1.2]) Para $x=\sum_{j=1}^{k} x_{j}^{1} \otimes \cdots \otimes x_{j}^{n} \in X_{1} \otimes \cdots \otimes X_{n}$, são equivalentes:

(i) $x=0$;

(ii) $\sum_{j=1}^{k} \varphi_{1}\left(x_{j}^{1}\right) \cdots \varphi_{n}\left(x_{j}^{n}\right)=0$ para quaisquer funcionais lineares $\varphi_{j}$ em $X_{j}, j=1, \ldots, n$.

O principal motivo da introdução do produto tensorial nesta tese está na linearização das aplicações multilineares, que apresentamos a seguir. Dados espaços vetoriais $X_{1}, \ldots, X_{n}$, segue da Proposição 1.4.1 que a aplicação

$$
\sigma_{n}: X_{1} \times \cdots \times X_{n} \longrightarrow X_{1} \otimes \cdots \otimes X_{n}, \sigma_{n}\left(x_{1}, \ldots, x_{n}\right)=x_{1} \otimes \cdots \otimes x_{n},
$$

é $n$-linear.

Teorema 1.4.3. (Veja [49, Proposition 1.4] ou [51, Teorema 1.10]) Para toda aplicação $n$-linear $A \in L\left(X_{1}, \ldots, X_{n} ; Y\right)$ existe um único operador linear $A_{L} \in L\left(X_{1} \otimes \cdots \otimes X_{n} ; Y\right)$ tal que

$$
A=A_{L} \circ \sigma_{n},
$$

isto é, o diagrama a seguir é comutativo: 


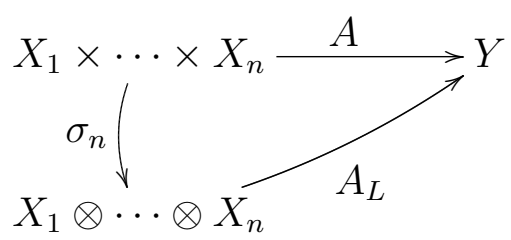

Além disso, a correspondência $A \longleftrightarrow A_{L}$ é um isomorfismo entre os espaços vetoriais $L\left(X_{1}, \ldots, X_{n} ; Y\right)$ e $L\left(X_{1} \otimes \cdots \otimes X_{n} ; Y\right)$.

Observação 1.4.4. (i) O operador linear $A_{L}$ é chamado de linearização da aplicação multilinear $A$.

(ii) Como $A_{L}$ é linear, única e $A_{L} \circ \sigma_{n}=A$, podemos obter sua expressão geral, que é

$$
A_{L}(x)=\sum_{j=1}^{k} A\left(x_{j}^{1}, \ldots, x_{j}^{n}\right),
$$

onde $x=\sum_{j=1}^{k} x_{j}^{1} \otimes \cdots \otimes x_{j}^{n}$. Da unicidade da linearização segue que essa soma independe da representação de $x$.

(iii) Em particular, se tomarmos $A=\sigma_{n}$ obtemos facilmente que $\left(\sigma_{n}\right)_{L}=I d_{X_{1} \otimes \cdots \otimes X_{n}}$ (por $I d_{X}$ denotamos o operador identidade em $X$ ).

É desejável que a linearização de uma aplicação multilinear contínua seja um operador linear contínuo e vice-versa. Para isso precisamos definir uma norma no produto tensorial de espaços normados.

Proposição 1.4.5. (Veja [49, Proposition 2.1] ou [51, Proposição 3.3]) Dados espaços normados $E_{1}, \ldots, E_{n}$, a função $\pi: E_{1} \otimes \cdots \otimes E_{n} \longrightarrow[0, \infty)$ dada por

$$
\pi(x)=\inf \left\{\sum_{j=1}^{k}\left\|x_{j}^{1}\right\| \cdots\left\|x_{j}^{n}\right\|\right\},
$$

onde o infimo é tomado sobre todas as representações $x=\sum_{j=1}^{k} x_{j}^{1} \otimes \cdots \otimes x_{j}^{n}$, é uma norma em $E_{1} \otimes \cdots \otimes E_{n}$. Além disso,

$$
\pi\left(x_{1} \otimes \cdots \otimes x_{n}\right)=\left\|x_{1}\right\| \cdots\left\|x_{n}\right\|
$$

para quaisquer $x_{1} \in E_{1}, \ldots, x_{n} \in E_{n}$. 
A norma definida acima é chamada de norma projetiva e o espaço normado resultante é denotado por $E_{1} \otimes_{\pi} \cdots \otimes_{\pi} E_{n}$. Esse espaço é completo se, e somente se, $E_{1}, \ldots, E_{n}$ são de dimensão finita (veja [49, Exercícios 2.4 e 2.5] e [51, Proposição 3.4]). Como pretendemos trabalhar com espaços de dimensão infinita, consideraremos o completamento $X_{1} \widehat{\otimes}_{\pi} \cdots \widehat{\otimes}_{\pi} X_{n}$ de $X_{1} \otimes_{\pi} \cdots \otimes_{\pi} X_{n}$ segundo a norma projetiva. O espaço completado é chamado de produto tensorial projetivo.

Dados espaços normados $E_{1}, \ldots, E_{n}$, de (1.2) segue que a aplicação $n$-linear canônica $\sigma_{n}: E_{1} \times \cdots \times E_{n} \longrightarrow E_{1} \otimes_{\pi} \cdots \otimes_{\pi} E_{n}$ é contínua e $\left\|\sigma_{n}\right\|=1$.

Agora podemos enunciar o principal resultado dessa seção: a versão contínua do Teorema 1.4.3, isto é, a linearização (contínua) de aplicações multilineares contínuas.

Proposição 1.4.6. (Veja [49, Theorem 2.9] ou [51, Teorema 3.17]) Sejam $E_{1}, \ldots, E_{n} e$ $F$ espaços de Banach. Para cada aplicação n-linear contínua $A \in \mathcal{L}\left(E_{1}, \ldots, E_{n} ; F\right)$ existe um único operador linear contínuo $A_{L} \in \mathcal{L}\left(E_{1} \widehat{\otimes}_{\pi} \cdots \widehat{\otimes}_{\pi} E_{n} ; F\right)$ tal que

$$
A=A_{L} \circ \sigma_{n}
$$

Além disso, a correspondência $A \longleftrightarrow A_{L}$ é um isomorfismo isométrico entre os espaços de Banach $\mathcal{L}\left(E_{1}, \ldots, E_{n} ; F\right)$ e $\mathcal{L}\left(E_{1} \widehat{\otimes}_{\pi} \cdots \widehat{\otimes}_{\pi} E_{n} ; F\right)$.

\subsection{Ideais de aplicações multilineares}

Começamos com a noção de ideais de operadores (lineares), que foi introduzida por A. Pietsch como uma unificação de várias classes especiais de operadores lineares que eram estudadas individualmente. Para a teoria de ideais de operadores nos referimos a $[21,43]$.

Definição 1.5.1. Um ideal de operadores é uma subclasse $\mathcal{I}$ da classe dos operadores lineares contínuos entre espaços de Banach tal que suas componentes

$$
\mathcal{I}(E ; F):=\mathcal{L}(E ; F) \cap \mathcal{I}
$$

onde $E$ e $F$ são espaços de Banach arbitrários, satisfazem as seguintes condições:

(1) $\mathcal{I}(E ; F)$ é um subespaço vetorial de $\mathcal{L}(E ; F)$ que contém os operadores lineares contínuos de posto finito;

(2) A propriedade de ideal: se $u_{1} \in \mathcal{L}(G ; E), u_{2} \in \mathcal{I}(E ; F)$ e $u_{3} \in \mathcal{L}(F ; H)$, então a composição $u_{3} \circ u_{2} \circ u_{1}$ pertence a $\mathcal{I}(G ; H)$. 
Se existem $0<p \leq 1$ e uma função $\|\cdot\|_{\mathcal{I}}: \mathcal{I} \longrightarrow[0, \infty)$ tais que:

(a) A função $\|\cdot\|_{\mathcal{I}}$ restrita à componente $\mathcal{I}(E ; F)$ é uma $p$-norma para quaisquer espaços de Banach $E$ e $F$;

(b) O funcional $I d_{\mathbb{K}}: \mathbb{K} \longrightarrow \mathbb{K}$, dado por $I d_{\mathbb{K}}(\lambda)=\lambda$, é tal que $\left\|I d_{\mathbb{K}}\right\|_{\mathcal{I}}=1$;

(c) Se $u_{1} \in \mathcal{L}(G ; E), u_{2} \in \mathcal{I}(E ; F)$ e $u_{3} \in \mathcal{L}(F ; H)$, então a composição satisfaz

$$
\left\|u_{3} \circ u_{2} \circ u_{1}\right\|_{\mathcal{I}} \leq\left\|u_{3}\right\| \cdot\left\|u_{2}\right\|_{\mathcal{I}} \cdot\left\|u_{1}\right\|
$$

então $\left(\mathcal{I} ;\|\cdot\|_{\mathcal{I}}\right)$ é um ideal p-normado de operadores.

Mais ainda, se todas as componentes $\mathcal{I}(E ; F)$ forem espaços completos relativamente à topologia gerada pela $p$-norma $\|\cdot\|_{\mathcal{I}}$, então dizemos que $\left(\mathcal{I} ;\|\cdot\|_{\mathcal{I}}\right)$ é um ideal p-Banach. No caso $p=1$ escrevemos apenas ideal normado e ideal de Banach.

Muitas classes clássicas de operadores lineares são ideais de operadores, por exemplo: operadores de posto finito, operadores aproximáveis, operadores nucleares, operadores compactos, operadores fracamente compactos, operadores completamente contínuos e operadores absolutamente $p$-somantes.

Em 1983, o próprio Pietsch iniciou em [44] a teoria de ideais de aplicações multilineares:

Definição 1.5.2. Um ideal de aplicações multilineares, ou simplesmente multi-ideal, é uma subclasse $\mathcal{M}$ da classe das aplicações multilineares contínuas entre espaços de Banach tal que suas componentes

$$
\mathcal{M}\left(E_{1}, \ldots, E_{n} ; F\right):=\mathcal{L}\left(E_{1}, \ldots, E_{n} ; F\right) \cap \mathcal{M}
$$

onde $n$ é um inteiro positivo qualquer e $E_{1}, \ldots, E_{n}$ e $F$ são espaços de Banach arbitrários, satisfazem as seguintes condições:

(1) $\mathcal{M}\left(E_{1}, \ldots, E_{n} ; F\right)$ é um subespaço vetorial de $\mathcal{L}\left(E_{1}, \ldots, E_{n} ; F\right)$ que contém as aplicações $n$-lineares de tipo finito;

(2) A propriedade de multi-ideal: se $A \in \mathcal{M}\left(E_{1}, \ldots, E_{n} ; F\right), u_{j} \in \mathcal{L}\left(G_{j} ; E_{j}\right), j=$ $1, \ldots, n$, e $t \in \mathcal{L}(F ; H)$, então a composição to $A \circ\left(u_{1}, \ldots, u_{n}\right)$ pertence a $\mathcal{M}\left(G_{1}, \ldots, G_{n} ; H\right)$.

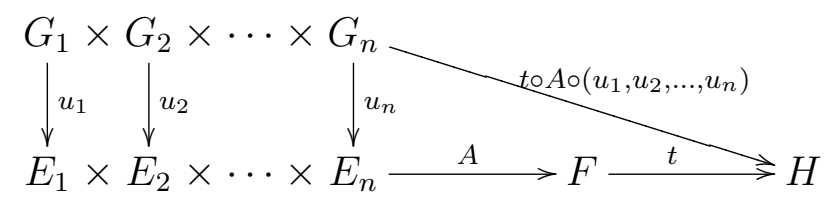


Se existem $0<p \leq 1$ e uma função $\|\cdot\|_{\mathcal{M}}: \mathcal{M} \longrightarrow[0, \infty)$ tais que:

(a) A função $\|\cdot\|_{\mathcal{M}}$ restrita à componente $\mathcal{M}\left(E_{1}, \ldots, E_{n} ; F\right)$ é uma $p$-norma para todo $n \in \mathbb{N}$ e quaisquer espaços de Banach $E_{1}, \ldots, E_{n}, F$

(b) A aplicação $n$-linear $I_{n}: \mathbb{K}^{n} \longrightarrow \mathbb{K}$, dada por $I_{n}\left(\lambda_{1}, \ldots, \lambda_{n}\right)=\lambda_{1} \cdots \lambda_{n}$, é tal que $\left\|I_{n}\right\|_{\mathcal{M}}=1$

(c) Se $A \in \mathcal{M}\left(E_{1}, \ldots, E_{n} ; F\right), u_{j} \in \mathcal{L}\left(G_{j} ; E_{j}\right), j=1, \ldots, n$ e $t \in \mathcal{L}(F ; H)$, então a composta satisfaz

$$
\left\|t \circ A \circ\left(u_{1}, \ldots, u_{n}\right)\right\|_{\mathcal{M}} \leq\|t\| \cdot\|A\|_{\mathcal{M}} \cdot\left\|u_{1}\right\| \cdots\left\|u_{n}\right\|,
$$

então $\left(\mathcal{M} ;\|\cdot\|_{\mathcal{M}}\right)$ é chamado de multi-ideal p-normado.

Mais ainda, se todas as componentes $\mathcal{M}\left(E_{1}, \ldots, E_{n} ; F\right)$ são subespaços completos relativamente à topologia gerada pela $p$-norma $\|\cdot\|_{\mathcal{M}}$, então dizemos que $\left(\mathcal{M} ;\|\cdot\|_{\mathcal{M}}\right)$ é um multi-ideal $p$-Banach. No caso $p=1$ escrevemos simplesmente multi-ideal normado e multi-ideal de Banach.

Muitas classes clássicas de aplicações multilineares são multi-ideais, por exemplo: aplicações multilineares de tipo finito, de posto finito, aproximáveis por aplicações de tipo finito, aproximáveis por aplicações de posto finito, nucleares, compactas, fracamente compactas, fracamente sequencialmente contínuas na origem, $\left(p_{1}, \ldots, p_{n}\right)$-dominadas, etc.

O seguinte critério para verificar que determinada classe é um multi-ideal $p$-Banach, o qual é uma generalização do Critério da Série para ideais de operadores (veja [21, 9.4] ou [43, 6.2.3]), é muito útil:

Teorema 1.5.3. [Critério da Série] (Veja [16, Proposition 2.1.5] ou [30, Criterion]) Seja $0<p \leq 1$. Uma subclasse $\mathcal{M}$ da classe das aplicações multilineares contínuas entre espaços de Banach com uma função $\|\cdot\|_{\mathcal{M}}: \mathcal{M} \longrightarrow[0, \infty)$ é um multi-ideal p-Banach se, e somente se, as seguintes condições estão satisfeitas:

(i) $\mathcal{M}$ satisfaz a condição (2) da Definição 1.5.2;

(ii) $I_{n} \in \mathcal{M}$ para todo $n \in \mathbb{N}$;

(iii) $\|\cdot\|_{\mathcal{M}}$ satifaz as condições (b) e (c) da Definição 1.5.2;

(iv) $S e\left(A_{j}\right)_{j=1}^{\infty} \subseteq \mathcal{M}\left(E_{1}, \ldots, E_{n} ; F\right) e \sum_{j=1}^{\infty}\left\|A_{j}\right\|_{\mathcal{M}}^{p}<\infty$, então

$$
A:=\sum_{j=1}^{\infty} A_{j} \in \mathcal{M}\left(E_{1}, \ldots, E_{n} ; F\right) \text { e }\|A\|_{\mathcal{M}}^{p} \leq \sum_{j=1}^{\infty}\left\|A_{j}\right\|_{\mathcal{M}}^{p} .
$$


A seguir descreveremos dois métodos para gerar multi-ideais a partir de ideais de operadores. Tais métodos servirão de inspiração na busca por resultados similares no próximo capítulo.

Definição 1.5.4 (Método da fatoração). Sejam $\mathcal{I}_{j}$ ideais de operadores, $j=1,2, \ldots, n$. Diz-se que uma aplicação $A \in \mathcal{L}\left(E_{1}, \ldots, E_{n} ; F\right)$ pertence a $\mathcal{L}\left(\mathcal{I}_{1}, \ldots, \mathcal{I}_{n}\right)\left(E_{1}, \ldots, E_{n} ; F\right)$ se existem espaços de Banach $G_{1}, \ldots, G_{n}$, operadores lineares $u_{j} \in \mathcal{I}_{j}\left(E_{j} ; G_{j}\right), j=1,2, \ldots, n$ e uma aplicação $n$-linear contínua $B \in \mathcal{L}\left(G_{1}, \ldots, G_{n} ; F\right)$ tais que

$$
A=B \circ\left(u_{1}, \ldots, u_{n}\right)
$$

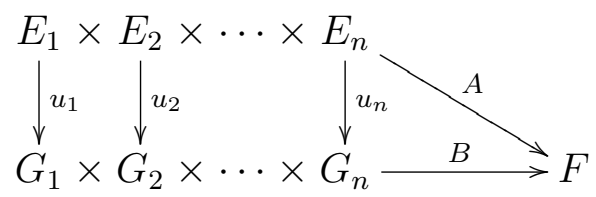

Se cada $\mathcal{I}_{j}$ é ideal $p_{j}$-normado, definimos ainda

$$
\|A\|_{\mathcal{L}\left(\mathcal{I}_{1}, \ldots, \mathcal{I}_{n}\right)}=\inf \left\{\|B\| \cdot\left\|u_{1}\right\|_{\mathcal{I}_{1}} \cdots\left\|u_{n}\right\|_{\mathcal{I}_{n}}\right\}
$$

onde o ínfimo é tomado sobre todas as possíveis fatorações $A=B \circ\left(u_{1}, \ldots, u_{n}\right)$ com $u_{j} \in \mathcal{I}_{j}, j=1, \ldots, n$. Se $\mathcal{I}_{1}=\cdots=\mathcal{I}_{n}=\mathcal{I}$, escreveremos $\mathcal{L}(\mathcal{I})$ no lugar de $\mathcal{L}(\mathcal{I}, \ldots, \mathcal{I})$.

Para maiores informações sobre o método da fatoração veja $[7,16]$.

Definição 1.5.5 (Ideais de composição). Seja $\mathcal{I}$ um ideal de operadores. Diz-se que uma aplicação $A \in \mathcal{L}\left(E_{1}, \ldots, E_{n} ; F\right)$ pertence a $\mathcal{I} \circ \mathcal{L}\left(E_{1}, \ldots, E_{n} ; F\right)$ se existem um espaço de Banach $G$, um operador linear $u \in \mathcal{I}(G ; F)$ e uma aplicação $n$-linear $B \in \mathcal{L}\left(E_{1}, \ldots, E_{n} ; G\right)$ tais que

$$
A=u \circ B
$$

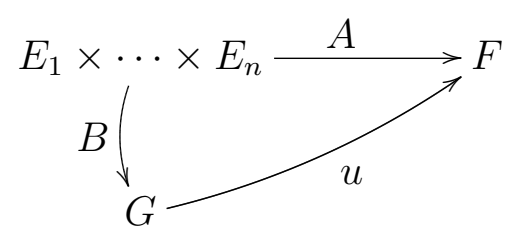

Se $\mathcal{I}$ é ideal $p$-normado, definimos ainda

$$
\|A\|_{\mathcal{I} \circ \mathcal{L}}=\inf \left\{\|u\|_{\mathcal{I}}\|B\|\right\}
$$

onde o ínfimo é tomado sobre todas as possíveis fatorações $A=u \circ B \operatorname{com} u \in \mathcal{I}$. 
Para maiores informações sobre ideais de composição veja [10]. Em particular, a caracterização a seguir é bastante útil:

Proposição 1.5.6. (Veja [10, Proposition 3.2 (a)]) Seja $\mathcal{I}$ um ideal de operadores. As seguintes afirmações são equivalentes para uma aplicação $n$-linear $A \in \mathcal{L}\left(E_{1}, \ldots, E_{n} ; F\right)$ :

(a) $A \in \mathcal{I} \circ \mathcal{L}\left(E_{1}, \ldots, E_{n} ; F\right)$;

(b) $A_{L} \in \mathcal{I}\left(E_{1} \widehat{\otimes}_{\pi} \cdots \widehat{\otimes}_{\pi} E_{n} ; F\right)$.

Proposição 1.5.7. (Veja [16, Proposition 2.3.3] e [10, Proposition 3.3]) Sejam $0<$ $p_{1}, \ldots, p_{n}, r \leq 1, \mathcal{I}_{j}$ ideal de operadores $p_{j}$-normado para $j=1,2, \ldots, n$, e $\mathcal{I}$ ideal de operadores r-normado. Então:

(a) $\mathcal{L}\left(\mathcal{I}_{1}, \ldots, \mathcal{I}_{n}\right)$ é um multi-ideal q-normado para $1 / q=1 / p_{1}+\cdots+1 / p_{n}$.

(b) $\mathcal{I} \circ \mathcal{L}$ é um multi-ideal r-normado.

Se os ideais $\mathcal{I}_{1}, \ldots, \mathcal{I}_{j}$ são completos, então o multi-ideal $\mathcal{L}\left(\mathcal{I}_{1}, \ldots, \mathcal{I}_{n}\right)$ é completo; e se o ideal $\mathcal{I}$ é completo, então o multi-ideal $\mathcal{I} \circ \mathcal{L}$ é completo.

Observação 1.5.8. Existe um terceiro método de gerar multi-ideais a partir de ideais de operadores, chamado de método da linearização (veja, por exemplo, [7, 9, 16]). Como este método não se mostrou útil para os propósitos deste trabalho, optamos por não introduzí-lo.

\subsection{Ideais de polinômios}

A noção de ideais de polinômios homogêneos é uma consequência natural do conceito de ideal de aplicações multilineares. Não se sabe precisamente quando o conceito foi formalmente introduzido, mas a ideia já se encontra na tese [15] de H.-A. Braunss de 1984.

Definição 1.6.1. Um ideal de polinômios homogêneos, ou simplesmente ideal de polinômios, é uma subclasse $\mathcal{Q}$ da classe dos polinômios homogêneos contínuos entre espaços de Banach tal que as suas componentes

$$
\mathcal{Q}\left({ }^{n} E ; F\right):=\mathcal{P}\left({ }^{n} E ; F\right) \cap \mathcal{Q},
$$

onde $n$ é um natural qualquer e $E$ e $F$ são espaços de Banach arbitrários, satisfazem as seguintes condições: 
(1) $\mathcal{Q}\left({ }^{n} E ; F\right)$ é um subespaço vetorial de $\mathcal{P}\left({ }^{n} E ; F\right)$ que contém os polinômios de tipo finito;

(2) A propriedade de ideal: se $P \in \mathcal{Q}\left({ }^{n} E ; F\right), u \in \mathcal{L}(G ; E)$ e $t \in \mathcal{L}(F ; H)$, então a composição $t \circ P \circ u$ pertence a $\mathcal{Q}\left({ }^{n} G ; H\right)$.

Se existem $0<p \leq 1$ e uma função $\|\cdot\|_{\mathcal{Q}}: \mathcal{Q} \longrightarrow[0, \infty)$ tais que:

(a) A função $\|\cdot\|_{\mathcal{Q}}$ restrita à componente $\mathcal{Q}\left({ }^{n} E ; F\right)$ é uma $p$-norma para quaisquer espaços de Banach $E, F$ e todo $n \in \mathbb{N}$;

(b) $\left\|P: \mathbb{K} \longrightarrow \mathbb{K}, P(x)=x^{n}\right\|_{\mathcal{Q}}=1$ para todo $n \in \mathbb{N}$;

(c) Se $u \in \mathcal{L}(G ; E), P \in \mathcal{Q}\left({ }^{n} E ; F\right)$ e $t \in \mathcal{L}(F ; H)$, então

$$
\|t \circ P \circ u\|_{\mathcal{Q}} \leq\|t\| \cdot\|P\|_{\mathcal{Q}} \cdot\|u\|^{n}
$$

então $\left(\mathcal{Q},\|\cdot\|_{\mathcal{Q}}\right)$ é chamado de ideal p-normado de polinômios.

Mais ainda, se todas as componentes $\mathcal{Q}\left({ }^{n} E ; F\right)$ são espaços completos relativamente à p-norma $\|\cdot\|_{\mathcal{Q}}$, dizemos que $\left(\mathcal{Q},\|\cdot\|_{\mathcal{Q}}\right)$ é um ideal p-Banach de polinômios. No caso $p=1$ escrevemos ideal normado de polinômios e ideal de Banach de polinômios.

Teorema 1.6.2 (Critério da Série para ideais de polinômios). (Veja [16, Proposition 2.4.5] ou [30, Criterion]) Uma subclasse $\mathcal{Q}$ da classe dos polinômios homogêneos contínuos entre espaços de Banach com uma função $\|\cdot\|_{\mathcal{Q}}: \mathcal{Q} \longrightarrow[0, \infty)$ é um ideal p-Banach de polinômios se, e somente se, as seguintes condições são verificadas:

(i) $\mathcal{Q}$ satisfaz a condição (2) da Definição 1.6.1;

(ii) O polinômio $P: \mathbb{K} \longrightarrow \mathbb{K}$ dado por $P(x)=x^{n}$ pertence a $\mathcal{Q}$ para todo $n$;

(iii) $\|\cdot\|_{\mathcal{Q}}$ satifaz (b) e (c) da Definição 1.6.1;

(iv) $S e\left(P_{j}\right)_{j=1}^{\infty} \subseteq \mathcal{Q}\left({ }^{n} E ; F\right) e \sum_{j=1}^{\infty}\left\|P_{j}\right\|_{\mathcal{Q}}^{p}<\infty$, então

$$
P:=\sum_{j=1}^{\infty} P_{j} \in \mathcal{Q}\left({ }^{n} E ; F\right) \text { e }\|P\|_{\mathcal{Q}}^{p} \leq \sum_{j=1}^{\infty}\left\|P_{j}\right\|_{\mathcal{Q}}^{p} .
$$

A seguir descrevemos dois métodos para gerar ideais de polinômios a partir de multiideais:

Proposição 1.6.3. (Veja $[7,16,31])$ Seja $\mathcal{M}$ um multi-ideal. Então as classes

$$
\mathcal{P}_{\mathcal{M}}:=\{P \in \mathcal{P}: \check{P} \in \mathcal{M}\} e
$$




$$
\mathcal{P}^{\mathcal{M}}:=\{P \in \mathcal{P}: \text { existe } A \in \mathcal{M} \text { tal que } \widehat{A}=P\}
$$

são ideais de polinômios. Além disso, se $\mathcal{M}$ é p-normado (digamos com p-norma $\|\cdot\|_{\mathcal{M}}$ ), então os ideais de polinômios $\mathcal{P}_{\mathcal{M}}$ e $\mathcal{P}^{\mathcal{M}}$ são p-normados quando munidos, respectivamente, das funções

$$
\begin{aligned}
& \|\cdot\|_{\mathcal{P}_{\mathcal{M}}}: \mathcal{P}_{\mathcal{M}} \longrightarrow[0, \infty),\|P\|_{\mathcal{P}_{\mathcal{M}}}:=\|\check{P}\|_{\mathcal{M}}, \\
& \|\cdot\|_{\mathcal{P}^{\mathcal{M}}}: \mathcal{P}_{\mathcal{M}} \longrightarrow[0, \infty),\|P\|_{\mathcal{P}^{\mathcal{M}}}:=\inf \left\{\|A\|_{\mathcal{M}}: A \in \mathcal{M} \text { e } \widehat{A}=P\right\}
\end{aligned}
$$

Dado um ideal de operadores $\mathcal{I}$, podemos aplicar os dois métodos acima para criar ideais de polinômios a partir do multi-ideal $\mathcal{L}(\mathcal{I})$ gerado pelo método da fatoração e a partir do multi-ideal de composição $\mathcal{I} \circ \mathcal{L}$. Os dois resultados a seguir dizem que, nesses dois casos, os ideais de polinômios resultantes são coincidentes:

Proposição 1.6.4 (Método da fatoração). (Veja [7, Proposition 9]) Sejam $\mathcal{I}$ ideal de operadores e $P \in \mathcal{P}\left({ }^{n} E ; F\right)$. São equivalentes:

(a) Existe $A \in \mathcal{L}(\mathcal{I})\left({ }^{n} E ; F\right)$ tal que $\widehat{A}=P$;

(b) Existem um espaço de Banach $G$, um operador linear $u \in \mathcal{I}(E ; G)$ e um polinômio $Q \in \mathcal{P}\left({ }^{n} G ; F\right)$ tais que $P=Q \circ u$;

(c) $\check{P} \in \mathcal{L}(\mathcal{I})\left({ }^{n} E ; F\right)$.

Denotamos por $\mathcal{P}_{\mathcal{L}(\mathcal{I})}$ o ideal de polinômios formado pelos polinômios que satisfazem as condições equivalentes da proposição anterior. No caso em que $\mathcal{I}$ é um ideal $p$-normado, de acordo com a Proposição $1.6 .3, \mathcal{P}_{\mathcal{L}(\mathcal{I})}$ se torna ideal $p$-normado de polinômios com qualquer uma das seguintes funções:

$$
\begin{gathered}
\|\cdot\|_{\mathcal{P}_{\mathcal{L}(\mathcal{I})}, 1}: \mathcal{P}_{\mathcal{L}(\mathcal{I})} \longrightarrow[0, \infty),\|P\|_{\mathcal{P}_{\mathcal{L}(\mathcal{I})}, 1}=\inf \left\{\|A\|_{\mathcal{L}(\mathcal{I})}: A \in \mathcal{L}(\mathcal{I}) \text { e } \widehat{A}=P\right\} \\
\|\cdot\|_{\mathcal{P}_{\mathcal{L}(\mathcal{I}), 2}:}: \mathcal{P}_{\mathcal{L}(\mathcal{I})} \longrightarrow[0, \infty),\|P\|_{\mathcal{P}_{\mathcal{L}(\mathcal{I})}, 2}=\|\check{P}\|_{\mathcal{L}(\mathcal{I})} .
\end{gathered}
$$

Por simplicidade denotamos $\widehat{\otimes}_{\pi}^{n} E$ no lugar de $E \widehat{\otimes}_{\pi} \cdot(n) \widehat{\otimes}_{\pi} E$.

Proposição 1.6.5 (Ideal de composição). (Veja [10, Proposition 3.2(b)]) Sejam $\mathcal{I}$ ideal de operadores e $P \in \mathcal{P}\left({ }^{n} E ; F\right)$. São equivalentes:

(a) Existem um espaço de Banach $G$, um polinômio $P \in \mathcal{P}\left({ }^{n} E ; G\right)$ e um operador $u \in \mathcal{I}(G ; F)$ tais que $P=u \circ Q$;

(b) $(\check{P})_{L} \in \mathcal{I}\left(\widehat{\otimes}_{\pi}^{n} E ; F\right)$; 
(c) $\check{P} \in \mathcal{I} \circ \mathcal{L}\left({ }^{n} E ; F\right) ;$

(d) Existe $A \in \mathcal{I} \circ \mathcal{L}\left({ }^{n} E ; F\right)$ tal que $\widehat{A}=P$.

Denotamos por $\mathcal{I} \circ \mathcal{P}$ o ideal de polinômios formado pelos polinômios que satisfazem as condições equivalentes da proposição anterior. No caso em que $\mathcal{I}$ é um ideal $p$-normado, de acordo com a Proposição 1.6.3, $\mathcal{I} \circ \mathcal{P}$ se torna ideal $p$-normado de polinômios com qualquer uma das seguintes funções:

$$
\begin{gathered}
\|\cdot\|_{\mathcal{I} \circ \mathcal{P}, 1}: \mathcal{I} \circ \mathcal{P} \longrightarrow[0, \infty),\|P\|_{\mathcal{I} \circ \mathcal{P}, 1}=\inf \left\{\|A\|_{\mathcal{I}^{\circ} \mathcal{L}}: A \in \mathcal{I} \circ \mathcal{L}\left({ }^{n} E ; F\right) \text { e } \widehat{A}=P\right\} \\
\|\cdot\|_{\mathcal{I} \circ \mathcal{P}, 2}: \mathcal{P}_{\mathcal{L}(\mathcal{I})} \longrightarrow[0, \infty),\|P\|_{\mathcal{I}^{\circ} \mathcal{P}, 2}=\|\check{P}\|_{\mathcal{I} \circ \mathcal{L}}
\end{gathered}
$$




\section{Capítulo 2}

\section{Hiper-ideais de Aplicações Multilineares}

\subsection{Definição e propriedades gerais}

A propriedade de multi-ideal (condição (2) da Definição 1.5.2) apresenta um aspecto aparentemente paradoxal: parte-se de uma aplicação multilinear e considera-se composições com operadores lineares, e não com aplicações multilineares. É claro que à direita só faz sentido compor com operadores lineares, mas à esquerda faz todo sentido compor com aplicações multilineares. O motivo pelo qual a definição original de Pietsch considera apenas a composição com operadores lineares é o seguinte: ao iniciar o processo com uma aplicação $n$-linear, deseja-se chegar ao final também com uma aplicação $n$-linear. Para isso é necessário compor apenas com operadores lineares, pois caso contrário, ou seja, compondo com aplicações multilineares, poderia-se chegar ao final com uma aplicação com um grau de multilinearidade maior que o da aplicação original. Este cuidado em começar e terminar mantendo o grau de multilinearidade justifica-se pelo procedimento usual de manter a natureza dos objetos que estão sendo tratados.

Entretanto, ao fazer a composição apenas com operadores lineares, o conceito de multiideal restringe a natureza multilinear do assunto. Se pensarmos que estamos tratando com aplicações multilineares, isto é, com aplicações $n$-lineares com $n$ arbitrário, e não com aplicações $n$-lineares com $n$ fixo, faz todo sentido compor com aplicações multilineares à esquerda e obter a composta com grau de multilinearidade maior que a aplicação original. Faz sentido também estudar classes que são estáveis em relação a esse tipo de composição, e isso já foi objeto de estudo de alguns especialistas, veja, por exemplo, [23, 46, 47]. O ob- 
jetivo central desta tese é explorar, em sua totalidade, a natureza multilinear do assunto, e para isso introduzimos e desenvolvemos a teoria de classes de aplicações multilineares que gozam deste tipo de estabilidade; as quais optamos por chamar de hiper-ideais de aplicações multilineares.

Esta estabilidade em relação à composição com aplicações multilineares à esquerda e operadores lineares à direita é formalizada da seguinte maneira:

Propriedade de hiper-ideal. Uma subclasse $\mathcal{H}$ da classe das aplicações multilineares contínuas entre espaços de Banach tem a propriedade de hiper-ideal se cumpre a seguinte condição: Dados números naturais $n$ e $1 \leq m_{1}<\cdots<m_{n}$, espaços de Banach $G_{1}, \ldots, G_{m_{n}}, E_{1}, \ldots, E_{n}, F$ e $H$, se $A \in \mathcal{H}\left(E_{1}, \ldots, E_{n} ; F\right), B_{1} \in \mathcal{L}\left(G_{1}, \ldots, G_{m_{1}} ; E_{1}\right), \ldots$, $B_{n} \in \mathcal{L}\left(G_{m_{n-1}+1}, \ldots, G_{m_{n}} ; E_{n}\right)$, e $t \in \mathcal{L}(F ; H)$, então a composta $t \circ A \circ\left(B_{1}, \ldots, B_{n}\right)$ pertence a $\mathcal{H}\left(G_{1}, \ldots, G_{m_{n}} ; H\right)$. Esta condição pode ser expressa na forma de diagrama da seguinte maneira:

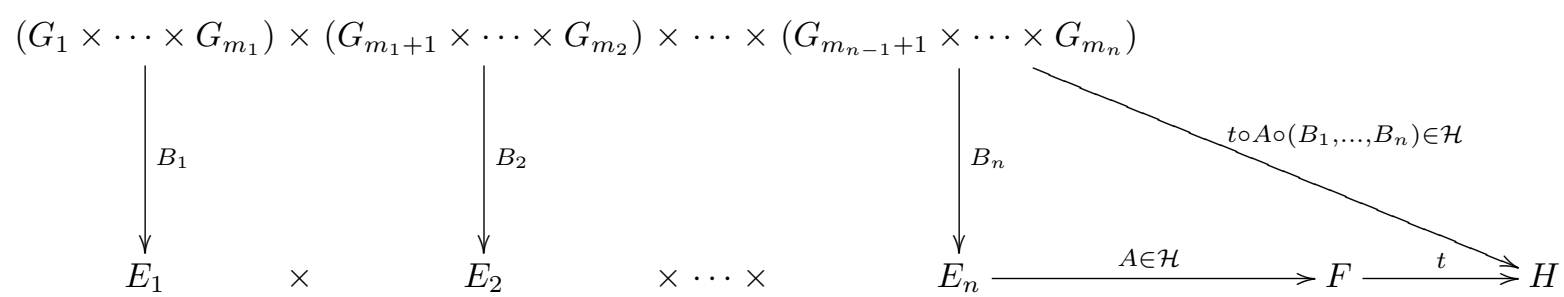

Conforme mencionado acima, ao executarmos este tipo de composição estaremos eventualmente aumentando o grau de multilinearidade da aplicação. Visando maior abrangência de exemplos, se faz necessário introduzir o conceito de $\left(p_{n}\right)_{n=1}^{\infty}$-norma:

Definição 2.1.1. Sejam $\left(p_{n}\right)_{n=1}^{\infty}$ uma sequência em $(0,1]$ e $\mathcal{H}$ uma subclasse da classe das aplicações multilineares contínuas entre espaços de Banach. Dizemos que uma função $\|\cdot\|_{\mathcal{H}}: \mathcal{H} \longrightarrow[0, \infty)$ é uma $\left(p_{n}\right)_{n=1}^{\infty}$-norma em $\mathcal{H}$ se, para cada natural $n$ e espaços de Banach arbitrários $E_{1}, \ldots, E_{n}, F,\|\cdot\|_{\mathcal{H}}$ restrita à componente

$$
\mathcal{H}\left(E_{1}, \ldots, E_{n} ; F\right):=\mathcal{L}\left(E_{1}, \ldots, E_{n} ; F\right) \cap \mathcal{H}
$$

é uma $p_{n}$-norma. No caso em que $p_{n}=1$ para todo $n$, dizemos simplesmente que $\|\cdot\|_{\mathcal{H}}$ é uma norma em $\mathcal{H}$.

Incorporando esses aspectos aos da definição de multi-ideais, obtemos o seguinte conceito:

Definição 2.1.2. Um hiper-ideal de aplicações multilineares, ou simplesmente hiper-ideal, é uma subclasse $\mathcal{H}$, da classe das aplicações multilineares contínuas entre espaços de 
Banach tal que, para quaisquer $n \in \mathbb{N}$ e espaços de Banach $E_{1}, \ldots, E_{n}, F$, as componentes

$$
\mathcal{H}\left(E_{1}, \ldots, E_{n} ; F\right):=\mathcal{L}\left(E_{1}, \ldots, E_{n} ; F\right) \cap \mathcal{H}
$$

satisfazem as seguintes condições:

(1) $\mathcal{H}\left(E_{1}, \ldots, E_{n} ; F\right)$ é um subespaço vetorial de $\mathcal{L}\left(E_{1}, \ldots, E_{n} ; F\right)$ que contém as aplicações $n$-lineares de tipo finito;

(2) Propriedade de hiper-ideal: Dados números naturais $n$ e $1 \leq m_{1}<\cdots<m_{n}$, espaços de Banach $G_{1}, \ldots, G_{m_{n}}, E_{1}, \ldots, E_{n}, F$ e $H$, se $B_{1} \in \mathcal{L}\left(G_{1}, \ldots, G_{m_{1}} ; E_{1}\right), \ldots, B_{n} \in$ $\mathcal{L}\left(G_{m_{n-1}+1}, \ldots, G_{m_{n}} ; E_{n}\right), t \in \mathcal{L}(F ; H)$ e $A \in \mathcal{H}\left(E_{1}, \ldots, E_{n} ; F\right)$, então a composta $t \circ A \circ$ $\left(B_{1}, \ldots, B_{n}\right)$ pertence a $\mathcal{H}\left(G_{1}, \ldots, G_{m_{n}} ; H\right)$.

Se existem uma sequência $\left(p_{n}\right)_{n=1}^{\infty}$ em $(0,1]$ e uma função $\|\cdot\|_{\mathcal{H}}: \mathcal{H} \longrightarrow[0, \infty)$ tais que:

(a) A função $\|\cdot\|_{\mathcal{H}}$ restrita à componente $\mathcal{H}\left(E_{1}, \ldots, E_{n} ; F\right)$ é uma $p_{n}$-norma para quaisquer espaços de Banach $E_{1}, \ldots, E_{n}, F$ e todo $n \in \mathbb{N}$;

(b) A aplicação $n$-linear $I_{n}: \mathbb{K}^{n} \longrightarrow \mathbb{K}$, dada por $I_{n}\left(\lambda_{1}, \ldots, \lambda_{n}\right)=\lambda_{1} \cdots \lambda_{n}$, é tal que $\left\|I_{n}\right\|_{\mathcal{H}}=1$

(c) Se $A \in \mathcal{H}\left(E_{1}, \ldots, E_{n} ; F\right), t \in \mathcal{L}(F ; H), B_{1} \in \mathcal{L}\left(G_{1}, \ldots, G_{m_{1}} ; E_{1}\right), \ldots, B_{n} \in$ $\mathcal{L}\left(G_{m_{n-1}+1}, \ldots, G_{m_{n}} ; E_{n}\right)$, então

$$
\left\|t \circ A \circ\left(B_{1}, \ldots, B_{n}\right)\right\|_{\mathcal{H}} \leq\|t\| \cdot\|A\|_{\mathcal{H}} \cdot\left\|B_{1}\right\| \cdots\left\|B_{n}\right\|,
$$

então $\left(\mathcal{H} ;\|\cdot\|_{\mathcal{H}}\right)$ é um hiper-ideal $\left(p_{n}\right)_{n=1}^{\infty}$-normado. Mais ainda, se todas as componentes $\mathcal{H}\left(E_{1}, \ldots, E_{n} ; F\right)$ são espaços completos relativamente à topologia gerada por $\|\cdot\|_{\mathcal{H}}$, dize$\operatorname{mos}$ que $\left(\mathcal{H} ;\|\cdot\|_{\mathcal{H}}\right)$ é um hiper-ideal $\left(p_{n}\right)_{n=1}^{\infty}$-Banach. Em particular, se $p_{n}=p$ para todo $n \in \mathbb{N}$ escrevemos simplesmente que $\left(\mathcal{H} ;\|\cdot\|_{\mathcal{H}}\right)$ é um hiper-ideal p-normado (p-Banach, respectivamente). Mais ainda, se $p=1$ dizemos que $\left(\mathcal{H} ;\|\cdot\|_{\mathcal{H}}\right)$ é um hiper-ideal normado (Banach, respectivamente).

Um hiper-ideal que é $\left(p_{n}\right)_{n=1}^{\infty}$-normado para alguma sequência $\left(p_{n}\right)_{n=1}^{\infty}$ em $(0,1]$ é chamado de hiper-ideal quasi-normado. Analogamente definimos hiper-ideal quasi-Banach.

Observação 2.1.3. ( $i$ ) Decorre da definição que todo hiper-ideal quasi-normado $(\mathcal{H}$; $\|$ • $\left.\|_{\mathcal{H}}\right)$ é um multi-ideal quasi-normado.

De fato, a condição (1) é a mesma para multi-ideais. Agora, tomando operadores lineares, que são casos particulares de aplicações multilineares, a propriedade de multi-ideal segue da propriedade de hiper-ideal. Analogamente, as condições $(a)$ e $(b)$ da Definição 
2.1.2 são as mesmas para multi-ideais; e a condição (c) segue novamente considerando-se operadores lineares no lugar de aplicações multilineares. Portanto $\left(\mathcal{H} ;\|\cdot\|_{\mathcal{H}}\right)$ é um multiideal $p$-normado.

(ii) Se uma classe $\mathcal{H}$ de aplicações multilineares satisfaz as condições (1) e (2) da definição acima, então $(\mathcal{H} ;\|\cdot\|)$ é um hiper-ideal normado com a norma uniforme $\|\cdot\|$.

(iii) Veremos, no Corolário 2.2.3, que, para hiper-ideais, a condição de conter os operadores de posto finito é equivalente a uma condição aparentemente mais exigente. Isso marcará uma importante diferença entre hiper-ideais e multi-ideais. Entretanto, podemos adiantar agora uma importante propriedade de hiper-ideais: o fato de incluir todas as formas $n$-lineares contínuas entre espaços de Banach.

De fato, sejam $\mathcal{H}$ um hiper-ideal e $T \in \mathcal{L}\left(E_{1}, \ldots, E_{n}\right)$. Pela condição (1) da Definição 2.1.2 temos $I d_{\mathbb{K}} \in \mathcal{H}(\mathbb{K})$. Agora, pela Propriedade de hiper-ideal,

$$
T=I d_{\mathbb{K}} \circ T \in \mathcal{H}\left(E_{1}, \ldots, E_{n}\right) .
$$

A partir de agora e até o final desta seção vamos provar algumas propriedades gerais de hiper-ideais. Isso tem duplo objetivo: (i) deixar prontos alguns fatos que serão necessários mais adiante, (ii) mostrar que a teoria de hiper-ideais satisfaz propriedades análogas, mutatis mutandis, às de multi-ideais e de ideais de operadores.

Tendo em vista o item (ii) da observação anterior podemos considerar a seguinte:

Definição 2.1.4. Seja $\mathcal{H}$ um hiper-ideal. O fecho de $\mathcal{H}$ é definido por

$$
\overline{\mathcal{H}}\left(E_{1}, \ldots, E_{n} ; F\right):=\overline{\mathcal{H}\left(E_{1}, \ldots, E_{n} ; F\right)} \|^{\|\cdot\|},
$$

para todos $n \in \mathbb{N}$ e $E_{1}, \ldots, E_{n}$ e $F$ espaços de Banach arbitrários. Denotamos essa classe por $\overline{\mathcal{H}}$.

Além disso, $\mathcal{H}$ é dito fechado se $\overline{\mathcal{H}}=\mathcal{H}$, ou seja, se $\mathcal{H}\left(E_{1}, \ldots, E_{n} ; F\right)$ é um subespaço fechado de $\mathcal{L}\left(E_{1}, \ldots, E_{n} ; F\right)$ em relação a norma uniforme, para quaisquer espaços de Banach $E_{1}, \ldots, E_{n}$ e $F$.

A respeito de fecho de hiper-ideais e hiper-ideais fechados temos a:

Proposição 2.1.5. Seja $\mathcal{H}$ um hiper-ideal. Então $(\overline{\mathcal{H}},\|\cdot\|)$ é um hiper-ideal de Banach. Mais ainda, $\overline{\mathcal{H}}$ é o menor hiper-ideal fechado que contém $\mathcal{H}$.

Demonstração. (1) Sendo $\mathcal{H}\left(E_{1}, \ldots, E_{n} ; F\right)$ um subespaço do espaço vetorial normado $\mathcal{L}\left(E_{1}, \ldots, E_{n} ; F\right)$, é imediato que $\overline{\mathcal{H}}\left(E_{1}, \ldots, E_{n} ; F\right)$ também é. Além disso, como $\mathcal{H} \subseteq \overline{\mathcal{H}}$ e $\mathcal{H}$ contém as aplicações de tipo finito, $\overline{\mathcal{H}}$ também as contém. 
(2) Dada $A \in \overline{\mathcal{H}}\left(E_{1}, \ldots, E_{n} ; F\right)$, existe uma sequência $\left(A_{j}\right)_{j=1}^{\infty} \subseteq \mathcal{H}\left(E_{1}, \ldots, E_{n} ; F\right)$ que converge para $A$ na norma uniforme. Sejam $1 \leq m_{1}<\cdots<m_{n}, B_{1} \in \mathcal{L}\left(G_{1}, \ldots, G_{m_{1}} ; E_{1}\right)$, $\ldots, B_{n} \in \mathcal{L}\left(G_{m_{n-1}+1}, \ldots, G_{m_{n}} ; E_{n}\right)$ e $t \in \mathcal{L}(F ; H)$. Então

$$
\begin{aligned}
\left\|t \circ A \circ\left(B_{1}, \ldots, B_{n}\right)-t \circ A_{j} \circ\left(B_{1}, \ldots, B_{n}\right)\right\| & =\left\|t \circ\left(A-A_{j}\right) \circ\left(B_{1}, \ldots, B_{n}\right)\right\| \\
& \leq\|t\| \cdot\left\|A-A_{j}\right\| \cdot\left\|B_{1}\right\| \cdots\left\|B_{n}\right\|,
\end{aligned}
$$

e portanto $\left(t \circ A_{j} \circ\left(B_{1}, \ldots, B_{n}\right)\right)_{j=1}^{\infty}$ converge para $t \circ A \circ\left(B_{1}, \ldots, B_{n}\right)$. Como $\mathcal{H}$ é hiperideal, temos $t \circ A_{j} \circ\left(B_{1}, \ldots, B_{n}\right) \in \mathcal{H}\left(G_{1}, \ldots, G_{m_{n}} ; H\right)$. Daí

$$
t \circ A \circ\left(B_{1}, \ldots, B_{n}\right) \in \overline{\mathcal{H}}\left(G_{1}, \ldots, G_{m_{n}} ; H\right)
$$

$\operatorname{Logo}(\mathcal{H},\|\cdot\|)$ é um hiper-ideal normado.

É claro que $\overline{\mathcal{H}}\left(E_{1}, \ldots, E_{n} ; F\right)$ é subespaço fechado do espaço de Banach $\mathcal{L}\left(E_{1}, \ldots, E_{n} ; F\right)$, logo $\overline{\mathcal{H}}\left(E_{1}, \ldots, E_{n} ; F\right)$ também é um espaço de Banach. Portanto $(\mathcal{H},\|\cdot\|)$ é um hiperideal de Banach.

Seja $\mathcal{G}$ hiper-ideal fechado que contém $\mathcal{H}$. Para quaisquer espaços de Banach $E_{1}, \ldots, E_{n}$ e $F$ e $A \in \overline{\mathcal{H}}\left(E_{1}, \ldots, E_{n} ; F\right)$ existe $\left(A_{j}\right)_{j=1}^{\infty} \subseteq \mathcal{H}\left(E_{1}, \ldots, E_{n} ; F\right) \subseteq \mathcal{G}\left(E_{1}, \ldots, E_{n} ; F\right)$ tal que $A_{j} \longrightarrow A$. Como $\mathcal{G}\left(E_{1}, \ldots, E_{n} ; F\right)$ é fechado, $A \in \mathcal{G}\left(E_{1}, \ldots, E_{n} ; F\right)$, provando que $\overline{\mathcal{H}}\left(E_{1}, \ldots, E_{n} ; F\right) \subseteq \mathcal{G}\left(E_{1}, \ldots, E_{n} ; F\right)$.

As proposições seguintes nos dizem que hiper-ideais quasi-normados satisfazem propriedades similares às do caso de multi-ideais quasi-normados.

Proposição 2.1.6. Seja $\left(\mathcal{H},\|\cdot\|_{\mathcal{H}}\right)$ um hiper-ideal $\left(p_{n}\right)_{n=1}^{\infty}$-normado. Então

$$
\|\cdot\| \leq\|\cdot\|_{\mathcal{H}}
$$

Demonstração. Seja $A \in \mathcal{H}\left(E_{1}, \ldots, E_{n} ; F\right)$. Dados $x_{j} \in E_{j}, j=1, \ldots, n$, pelo Teorema de Hahn-Banach existe um funcional $\varphi \in F^{\prime}$ tal que $\|\varphi\|=1 \mathrm{e}$

$$
\varphi\left(A\left(x_{1}, \ldots, x_{n}\right)\right)=\left\|A\left(x_{1}, \ldots, x_{n}\right)\right\| .
$$

Consideremos os operadores lineares

$$
\begin{gathered}
1 \otimes x_{i}: \mathbb{K} \longrightarrow E_{i}, 1 \otimes x_{i}(\lambda)=\lambda x_{i}, i=1, \ldots, n ; \mathrm{e} \\
\varphi: F \longrightarrow \mathbb{K} .
\end{gathered}
$$


De

$$
\begin{aligned}
\varphi \circ A \circ\left(1 \otimes x_{1}, \ldots, 1 \otimes x_{n}\right)\left(\lambda_{1}, \ldots, \lambda_{n}\right) & =\varphi \circ A\left(1 \otimes x_{1}\left(\lambda_{1}\right), \ldots, 1 \otimes x_{n}\left(\lambda_{n}\right)\right) \\
& =\varphi\left(A\left(\lambda_{1} x_{1}, \ldots, \lambda_{n} x_{n}\right)\right) \\
& =\lambda_{1} \cdots \lambda_{n} \varphi\left(A\left(x_{1}, \ldots, x_{n}\right)\right) \\
& =I_{n}\left(\lambda_{1}, \ldots, \lambda_{n}\right) \varphi\left(A\left(x_{1}, \ldots, x_{n}\right)\right),
\end{aligned}
$$

ou seja,

$$
\varphi \circ A \circ\left(1 \otimes x_{1}, \ldots, 1 \otimes x_{n}\right)=\varphi\left(A\left(x_{1}, \ldots, x_{n}\right)\right) \cdot I_{n}
$$

segue que

$$
\begin{aligned}
\left\|A\left(x_{1}, \ldots, x_{n}\right)\right\| & =\varphi\left(A\left(x_{1}, \ldots, x_{n}\right)\right)=\left|\varphi\left(A\left(x_{1}, \ldots, x_{n}\right)\right)\right| \cdot\left\|I_{n}\right\|_{\mathcal{H}} \\
& =\left\|\varphi\left(A\left(x_{1}, \ldots, x_{n}\right)\right) \cdot I_{n}\right\|_{\mathcal{H}}=\left\|\varphi \circ A \circ\left(1 \otimes x_{1}, \ldots, 1 \otimes x_{n}\right)\right\|_{\mathcal{H}} \\
& \leq\|\varphi\| \cdot\|A\|_{\mathcal{H}} \cdot\left\|1 \otimes x_{1}\right\| \cdots\left\|1 \otimes x_{n}\right\|=\|A\|_{\mathcal{H}} \cdot\left\|x_{1}\right\| \cdots\left\|x_{n}\right\| .
\end{aligned}
$$

Como os vetores $x_{i} \in E_{i}, i=1, \ldots, n$ são arbitrários, da desigualdade

$$
\left\|A\left(x_{1}, \ldots, x_{n}\right)\right\| \leq\|A\|_{\mathcal{H}} \cdot\left\|x_{1}\right\| \cdots\left\|x_{n}\right\|
$$

concluímos $\|A\| \leq\|A\|_{\mathcal{H}}$.

Observe que a demonstração acima é a mesma do caso de multi-ideais quasi-normados. Isso ocorre pois, como já vimos na Observação 2.1.3, todo hiper-ideal quasi-normado é um multi-ideal quasi-normado, e já é conhecido que a quasi-norma de um multi-ideal satisfaz a desigualdade desejada. Incluímos a demonstração visando a auto-suficiência do texto. Por outro lado, a proposição abaixo é mais geral que o resultado correspondente para multi-ideais:

Proposição 2.1.7. Sejam $\left(\mathcal{H},\|\cdot\|_{\mathcal{H}}\right)$ um hiper-ideal $\left(p_{n}\right)_{n=1}^{\infty}$-normado, $m_{1}<\cdots<m_{n}$ naturais, $T_{1} \in \mathcal{L}\left(E_{1}, \ldots, E_{m_{1}}\right), \ldots, T_{n} \in \mathcal{L}\left(E_{m_{n-1}+1}, \ldots, E_{m_{n}}\right)$ e $y \in F$. Consideremos a aplicação $m_{n}$-linear dada por

$$
T_{1} \otimes \cdots \otimes T_{n} \otimes y\left(x_{1}, \ldots, x_{m_{n}}\right)=T_{1}\left(x_{1}, \ldots, x_{m_{1}}\right) \cdots T_{n}\left(x_{m_{n-1}+1}, \ldots, x_{m_{n}}\right) \cdot y
$$

Então $T_{1} \otimes \cdots \otimes T_{n} \otimes y \in \mathcal{H}\left(E_{1}, \ldots, E_{m_{n}} ; F\right) e$

$$
\left\|T_{1} \otimes \cdots \otimes T_{n} \otimes y\right\|_{\mathcal{H}}=\left\|T_{1} \otimes \cdots \otimes T_{n} \otimes y\right\|=\left\|T_{1}\right\| \cdots\left\|T_{n}\right\| \cdot\|y\| .
$$


Demonstração. Considerando o operador linear $1 \otimes y: \mathbb{K} \longrightarrow F$ dado por $1 \otimes y(\lambda)=\lambda \cdot y$, temos

$$
\begin{aligned}
(1 \otimes y) \circ I_{m_{n}} \circ & \left(T_{1}, \ldots, T_{n}\right)\left(x_{1}, \ldots, x_{m_{n}}\right) \\
& =(1 \otimes y) \circ I_{m_{n}}\left(T_{1}\left(x_{1}, \ldots, x_{m_{1}}\right), \ldots, T_{n}\left(x_{m_{n-1}+1}, \ldots, x_{m_{n}}\right)\right) \\
& =(1 \otimes y)\left(T_{1}\left(x_{1}, \ldots, x_{m_{1}}\right) \cdots T_{n}\left(x_{m_{n-1}+1}, \ldots, x_{m_{n}}\right)\right) \\
& =T_{1}\left(x_{1}, \ldots, x_{m_{1}}\right) \cdots T_{n}\left(x_{m_{n-1}+1}, \ldots, x_{m_{n}}\right) y \\
& =T_{1} \otimes \cdots \otimes T_{n} \otimes y\left(x_{1}, \ldots, x_{m_{n}}\right) .
\end{aligned}
$$

Como $\mathcal{H}$ é um hiper-ideal e $I_{m_{n}} \in \mathcal{H}\left({ }^{m_{n}} \mathbb{K}\right)$, da propriedade de hiper-ideal segue que

$$
T_{1} \otimes \cdots \otimes T_{n} \otimes y=(1 \otimes y) \circ I_{m_{n}} \circ\left(T_{1}, \ldots, T_{n}\right) \in \mathcal{H}\left(E_{1}, \ldots, E_{m_{n}} ; F\right) .
$$

Além disso

$$
\begin{aligned}
\left\|T_{1} \otimes \cdots \otimes T_{n} \otimes y\right\| \\
=\sup _{\substack{\left\|x_{i}\right\| \leq 1 \leq 1 \\
i=1, \ldots, m_{n}}}\left\|T_{1} \otimes \cdots \otimes T_{n} \otimes y\left(x_{1}, \ldots, x_{m_{n}}\right)\right\| \\
=\sup _{\substack{\left\|x_{i}\right\| \leq 1 \\
i=1, \ldots, m_{n}}}\left|T_{1}\left(x_{1}, \ldots, x_{m_{1}}\right)\right| \cdots\left|T_{n}\left(x_{m_{n-1}+1}, \ldots, x_{m_{n}}\right)\right| \cdot\|y\| \\
=\left(\sup _{\substack{\left\|x_{i}\right\| \leq 1 \\
i=1, \ldots, m_{1}}}\left|T_{1}\left(x_{1}, \ldots, x_{m_{1}}\right)\right|\right) \cdots\left(\sup _{\substack{\left\|x_{i}\right\| \leq 1 \\
i=m_{n-1}+1, \ldots, m_{n}}}\left|T_{n}\left(x_{m_{n-1}+1}, \ldots, x_{m_{n}}\right)\right|\right) \cdot\|y\| \\
=\left\|T_{1}\right\| \cdots\left\|T_{n}\right\| \cdot\|y\| .
\end{aligned}
$$

Disso e da Proposição 2.1.6 obtemos

$$
\begin{aligned}
\left\|T_{1} \otimes \cdots \otimes T_{n} \otimes y\right\| & \leq\left\|T_{1} \otimes \cdots \otimes T_{n} \otimes y\right\|_{\mathcal{H}} \\
& =\left\|(1 \otimes y) \circ I_{m_{n}} \circ\left(T_{1}, \ldots, T_{n}\right)\right\|_{\mathcal{H}} \\
& \leq\|(1 \otimes y)\| \cdot\left\|I_{m_{n}}\right\|_{\mathcal{H}} \cdot\left\|T_{1}\right\| \cdots\left\|T_{n}\right\| \\
& =\left\|T_{1}\right\| \cdots\left\|T_{n}\right\| \cdot\|y\|=\left\|T_{1} \otimes \cdots \otimes T_{n} \otimes y\right\|,
\end{aligned}
$$

donde seguem as igualdades desejadas.

Observação 2.1.8. Notemos uma importante diferença com a teoria de multi-ideais. De acordo com a proposição acima, a quasi-norma de um hiper-ideal preserva a norma uniforme de formas multilineares quaisquer (e também de produtos de formas multilineares), 
enquanto que a quasi-norma de um multi-ideal preserva apenas a norma uniforme das formas multilineares que são produtos de funcionais lineares. Tal aspecto será importante mais adiante, por exemplo na demonstração da Proposição 2.2.2.

Teorema 2.1.9 (Critério da Série). Sejam $\left(p_{n}\right)_{n=1}^{\infty}$ uma sequência em $[0,1]$ e $\mathcal{H}$ uma subclasse da classe das aplicações multilineares contínuas entre espaços de Banach munida de uma função $\|\cdot\|_{\mathcal{H}}: \mathcal{H} \longrightarrow[0,+\infty)$. Então $\left(\mathcal{H},\|\cdot\|_{\mathcal{H}}\right)$ é um hiper-ideal $\left(p_{n}\right)_{n=1}^{\infty}$-Banach se, e somente se, as seguintes condições são satisfeitas:

(i) $I_{n} \in \mathcal{H}\left(\mathbb{K}^{n}\right)$ e $\left\|I_{n}\right\|_{\mathcal{H}}=1$ para todo $n \in \mathbb{N}$;

(ii) $S e\left(A_{j}\right)_{j=1}^{\infty} \subseteq \mathcal{H}\left(E_{1}, \ldots, E_{n} ; F\right) e \sum_{j=1}^{\infty}\left\|A_{j}\right\|_{\mathcal{H}}^{p_{n}}<\infty$, então

$$
A:=\sum_{j=1}^{\infty} A_{j} \in \mathcal{H}\left(E_{1}, \ldots, E_{n} ; F\right) e\|A\|_{\mathcal{H}}^{p_{n}} \leq \sum_{j=1}^{\infty}\left\|A_{j}\right\|_{\mathcal{H}}^{p_{n}}
$$

(iii) Se $1 \leq m_{1}<\cdots<m_{n}, G_{1}, \ldots, G_{m_{n}}, E_{1}, \ldots, E_{n}, F, H$ são espaços de Banach, $B_{1} \in \mathcal{L}\left(G_{1}, \ldots, G_{m_{1}} ; E_{1}\right), \ldots, B_{n} \in \mathcal{L}\left(G_{m_{n-1}+1}, \ldots, G_{m_{n}} ; E_{n}\right), A \in \mathcal{H}\left(E_{1}, \ldots, E_{n} ; F\right) e$ $t \in \mathcal{L}(F ; H)$, então $t \circ A \circ\left(B_{1}, \ldots, B_{n}\right) \in \mathcal{H}\left(G_{1}, \ldots, G_{m_{n}} ; H\right) e$

$$
\left\|t \circ A \circ\left(B_{1}, \ldots, B_{n}\right)\right\|_{\mathcal{H}} \leq\|t\| \cdot\|A\|_{\mathcal{H}} \cdot\left\|B_{1}\right\| \cdots\left\|B_{n}\right\|
$$

Demonstração. Primeiramente mostremos que as condições $(i)$, (ii) e (iii) implicam em $\left(\mathcal{H},\|\cdot\|_{\mathcal{H}}\right)$ ser um hiper-ideal $\left(p_{n}\right)_{n=1}^{\infty}$-Banach. Fixemos $n \in \mathbb{N}$ e $E_{1}, \ldots, E_{n}$ e $F$ espaços de Banach. Se $A, B \in \mathcal{H}\left(E_{1}, \ldots, E_{n} ; F\right)$, então $(i i)$ nos diz que $A+B \in \mathcal{H}\left(E_{1}, \ldots, E_{n} ; F\right)$ e (iii) que $\lambda A \in \mathcal{H}\left(E_{1}, \ldots, E_{n} ; F\right)$ para todo $\lambda \in \mathbb{K}$. Isso prova que $\mathcal{H}\left(E_{1}, \ldots, E_{n} ; F\right)$ é um subespaço vetorial de $\mathcal{L}\left(E_{1}, \ldots, E_{n} ; F\right)$.

Para mostrarmos que $\mathcal{H}\left(E_{1}, \ldots, E_{n} ; F\right)$ contém as aplicações de tipo finito, basta garantirmos que as aplicações do tipo $A=\varphi_{1} \otimes \cdots \otimes \varphi_{n} \otimes y$ pertencem a $\mathcal{H}\left(E_{1}, \ldots, E_{n} ; F\right)$, onde $\varphi_{i} \in E_{i}^{\prime}, i=1, \ldots, n$ e $y \in F$, pois sabemos que $\mathcal{H}\left(E_{1}, \ldots, E_{n} ; F\right)$ é espaço vetorial. Para isso escrevemos

$$
A=(1 \otimes y) \circ I_{n} \circ\left(\varphi_{1}, \ldots, \varphi_{n}\right),
$$

onde $1 \otimes y: \mathbb{K} \longrightarrow F$ é dada por $(1 \otimes y)(\lambda)=\lambda y$. Por $(i), I_{n} \in \mathcal{H}\left(\mathbb{K}^{n}\right)$, e por $(i i i)$

$$
A=(1 \otimes y) \circ I_{n} \circ\left(\varphi_{1}, \ldots, \varphi_{n}\right) \in \mathcal{H}\left(E_{1}, \ldots, E_{n} ; F\right) .
$$

Com isso verificamos a condição (1) da Definição 2.1.2. Note que a condição (2) está incluída em $(i i i)$. 
Agora devemos garantir que a funçao $\|\cdot\|_{\mathcal{H}}$ restrita a $\mathcal{H}\left(E_{1}, \ldots, E_{n} ; F\right)$ é uma $p_{n^{-}}$ norma. De $\left(\right.$ iii) segue que $\|\lambda A\|_{\mathcal{H}} \leq|\lambda| \cdot\|A\|_{\mathcal{H}}$ para todos $A \in \mathcal{H}\left(E_{1}, \ldots, E_{n} ; F\right)$ e $\lambda \in \mathbb{K}$. Para $\lambda=0$ a igualdade é clara, e para $\lambda \neq 0$,

$$
\|A\|_{\mathcal{H}}=\left\|\frac{1}{\lambda}(\lambda A)\right\|_{\mathcal{H}} \leq \frac{1}{|\lambda|} \cdot\|\lambda A\|_{\mathcal{H}} \Longrightarrow|\lambda| \cdot\|A\|_{\mathcal{H}} \leq\|\lambda A\|_{\mathcal{H}} .
$$

Assim $\|\lambda A\|_{\mathcal{H}}=|\lambda| \cdot\|A\|_{\mathcal{H}}$ para $\lambda \in \mathbb{K}$

Do que acabamos de provar segue que, se $A=0$, então

$$
\|0\|_{\mathcal{H}}=\|\lambda 0\|_{\mathcal{H}}=|\lambda| \cdot\|0\|_{\mathcal{H}}
$$

para todo $\lambda \in \mathbb{K}$. Em particular, para $\lambda=0$ obtemos $\|0\|_{\mathcal{H}}=0$. Reciprocamente, se $A \in \mathcal{H}\left(E_{1}, \ldots, E_{n} ; F\right)$ é tal que $\|A\|_{\mathcal{H}}=0$, tem-se

$$
\|j A\|_{\mathcal{H}}=j\|A\|_{\mathcal{H}}=0
$$

para todo $j \in \mathbb{N}$. De $(i i)$ segue que

$$
B:=\sum_{j=1}^{\infty} j A \in \mathcal{H}\left(E_{1}, \ldots, E_{n} ; F\right) .
$$

Se $A\left(x_{1}, \ldots, x_{n}\right) \neq 0$ para algum $\left(x_{1}, \ldots, x_{n}\right) \in E_{1} \times \cdots \times E_{n}$, então $B\left(x_{1}, \ldots, x_{n}\right)$ não estaria nem bem definida. Isso prova que $A=0$.

Por último, a desigualdade $p_{n}$-triangular segue imediatamente de $(i i)$. Isso completa a verificação de $(a)$ da Definição 2.1.2. Notemos ainda que $(i)$ e $(i i i)$ incluem as condições $(b)$ e $(c)$ da definição supracitada, respectivamente. Resta então mostrar que $\mathcal{H}\left(E_{1}, \ldots, E_{n} ; F\right)$ é completo. Para isso seja $\left(A_{j}\right)_{j=1}^{\infty} \subseteq \mathcal{H}\left(E_{1}, \ldots, E_{n} ; F\right)$ uma sequência de Cauchy com respeito a $\|\cdot\|_{\mathcal{H}}$. Existe uma subsequência $\left(A_{j_{k}}\right)_{k=1}^{\infty}$ de $\left(A_{j}\right)_{j=1}^{\infty}$ tal que

$$
\left\|A_{j_{k+1}}-A_{j_{k}}\right\|_{\mathcal{H}}^{p_{m}} \leq 2^{-k} \text { para todo } k \in \mathbb{N} .
$$

Então $\sum_{k=1}^{\infty}\left\|A_{j_{k+1}}-A_{j_{k}}\right\|_{\mathcal{H}}^{p_{n}}<\infty$, e por (ii) segue que

$$
B_{i}:=\sum_{k=i}^{\infty}\left(A_{j_{k+1}}-A_{j_{k}}\right) \in \mathcal{H}\left(E_{1}, \ldots, E_{n} ; F\right)
$$

e

$$
\left\|B_{i}\right\|_{\mathcal{H}}^{p_{n}} \leq \sum_{k=i}^{\infty}\left\|A_{j_{k+1}}-A_{j_{k}}\right\|_{\mathcal{H}}^{p_{n}} \leq 2^{-i+1} \text { para todo } i \in \mathbb{N}
$$


De

$$
B_{1}-B_{i}=\sum_{k=1}^{i-1}\left(A_{j_{k+1}}-A_{j_{k}}\right)=A_{j_{i}}-A_{j_{1}}
$$

obtemos $B_{i}=\left(B_{1}+A_{j_{1}}\right)-A_{j_{i}}$, e daí

$$
\left\|A_{j_{i}}-\left(B_{1}+A_{j_{1}}\right)\right\|_{\mathcal{H}}^{p_{n}}=\left\|B_{i}\right\|_{\mathcal{H}}^{p_{n}} \leq 2^{-i+1}
$$

Isso prova que $\left(A_{j}\right)_{j=1}^{\infty}$ possui uma subsequência convergente, logo $\left(A_{j}\right)_{j=1}^{\infty}$ também é convergente pois é de Cauchy.

Suponhamos agora que $\left(\mathcal{H},\|\cdot\|_{\mathcal{H}}\right)$ é um hiper-ideal $\left(p_{n}\right)_{n=1}^{\infty}$-Banach, e verifiquemos que as condições $(i),($ ii $),($ iii $)$ estão satisfeitas:

(i) Como $\mathcal{H}$ contém as aplicações de tipo finito, em particular $I_{n} \in \mathcal{H}\left(\mathbb{K}^{n}\right)$ para todo $n \in \mathbb{N}$. A condição $(b)$ da Definição 2.1.2 diz que $\left\|I_{n}\right\|_{\mathcal{H}}=1$.

(ii) Seja $\left(A_{j}\right)_{j=1}^{\infty} \subseteq \mathcal{H}\left(E_{1}, \ldots, E_{n} ; F\right)$ tal que $\sum_{j=1}^{\infty}\left\|A_{j}\right\|_{\mathcal{H}}^{p_{n}}<\infty$. Chamando de $\left(B_{j}\right)_{j=1}^{\infty}$ a sequência das somas parciais de $\left(A_{j}\right)_{j=1}^{\infty}$, por hipótese segue que

$$
\left(B_{j}\right)_{j=1}^{\infty} \subseteq \mathcal{H}\left(E_{1}, \ldots, E_{n} ; F\right)
$$

e que esta sequência é de Cauchy. Vejamos que, por completude $\left(B_{j}\right)_{j=1}^{\infty}$ converge para $\sum_{j=1}^{\infty} A_{j} \in \mathcal{H}\left(E_{1}, \ldots, E_{n} ; F\right)$. De fato, chamando de $A$ o limite da sequência $\left(B_{j}\right)_{j=1}^{\infty}$ em relação a $\|\cdot\|_{\mathcal{H}}$, temos $A \in \mathcal{H}\left(E_{1}, \ldots, E_{n} ; F\right)$ pois $\mathcal{H}$ é $\left(p_{n}\right)_{n=1}^{\infty}$-Banach. Agora

$$
\left\|B_{j}-A\right\|^{p_{n}} \leq\left\|B_{j}-A\right\|_{\mathcal{H}}^{p_{n}}
$$

para todo $j$, então $\left(B_{j}\right)_{j=1}^{\infty}$ também converge para $A$ na norma uniforme. Mas, nessa norma, $\left(B_{j}\right)_{j=1}^{\infty}$ converge para $\sum_{j=1}^{\infty} A_{j}$. Assim $A=\sum_{j=1}^{\infty} A_{j}$. Além disso, para todo $s$,

$$
\left\|\sum_{j=1}^{\infty} A_{j}\right\|_{\mathcal{H}}^{p_{n}} \leq\left\|\sum_{j=1}^{s} A_{j}\right\|_{\mathcal{H}}^{p_{n}}+\left\|\sum_{j=s+1}^{\infty} A_{j}\right\|_{\mathcal{H}}^{p_{n}} \leq \sum_{j=1}^{s}\left\|A_{j}\right\|_{\mathcal{H}}^{p_{n}}+\left\|\sum_{j=s+1}^{\infty} A_{j}\right\|_{\mathcal{H}}^{p_{n}} .
$$

Fazendo $s \longrightarrow \infty$ segue que $\|A\|_{\mathcal{H}}^{p_{n}} \leq \sum_{j=1}^{\infty}\left\|A_{j}\right\|_{\mathcal{H}}^{p_{n}}$.

A condição (iii) segue das condições (2) e (c) da Definição 2.1.2.

Assim como no caso de multi-ideais (veja [16, Proposition 2.1.6]), vale o seguinte resultado: 
Proposição 2.1.10. Sejam $\left(\mathcal{G},\|\cdot\|_{\mathcal{G}}\right)$ e $\left(\mathcal{H},\|\cdot\|_{\mathcal{H}}\right)$ hiper-ideais $\left(p_{n}\right)_{n=1}^{\infty}$-Banach tais que $\mathcal{G} \subseteq \mathcal{H}$. Então, para cada $n \in \mathbb{N}$ existe uma constante $C_{n}$, dependendo apenas de $n$, tal que

$$
\|A\|_{\mathcal{H}} \leq C_{n}\|A\|_{\mathcal{G}}
$$

para todos espaços de Banach $E_{1}, \ldots, E_{n}, F$ e $A \in \mathcal{G}\left(E_{1}, \ldots, E_{n} ; F\right)$.

Demonstração. Suponhamos que o resultado não seja verdadeiro. Neste caso existe $m \in \mathbb{N}$ para o qual não é possível encontrar uma tal constante $C_{m}$. Isso significa que, para cada $n \in \mathbb{N}$, existem espaços de Banach $E_{1}^{(n)}, \ldots, E_{m}^{(n)}, G_{n}$ e uma aplicação $A_{n} \in \mathcal{G}\left(E_{1}^{(n)}, \ldots, E_{m}^{(n)} ; G_{n}\right)$ tais que $\left\|A_{n}\right\|_{\mathcal{H}}>2^{n / p_{m}} n\left\|A_{n}\right\|_{\mathcal{G}}$. Vejamos que podemos supor, sem perda de generalidade, que $\left\|A_{n}\right\|_{\mathcal{G}}=\frac{1}{2^{n / p_{m}}}$. De fato, como $A_{n} \neq 0$, se tomarmos $\lambda=\frac{1}{2^{n / p_{m}}\left\|A_{n}\right\|_{\mathcal{G}}}$, temos

$$
\left\|\lambda A_{n}\right\|_{\mathcal{G}}=|\lambda| \cdot\left\|A_{n}\right\|_{\mathcal{G}}=\frac{\left\|A_{n}\right\|_{\mathcal{G}}}{2^{n / p_{m}}\left\|A_{n}\right\|_{\mathcal{G}}}=\frac{1}{2^{n / p_{m}}}
$$

e, além disso,

$$
\left\|\lambda A_{n}\right\|_{\mathcal{H}}=|\lambda| \cdot\left\|A_{n}\right\|_{\mathcal{H}}>|\lambda| \cdot 2^{n / p_{m}} \cdot n \cdot\left\|A_{n}\right\|_{\mathcal{G}}=2^{n / p_{m}} \cdot n \cdot\left\|\lambda A_{n}\right\|_{\mathcal{G}}=n .
$$

Sejam $E_{i}=\left(\bigoplus_{n=1}^{\infty} E_{i}^{(n)}\right)_{1}, i=1, \ldots, m$, e $G=\left(\bigoplus_{n=1}^{\infty} G_{n}\right)_{\infty}$. Consideremos ainda $\pi_{i n}: E_{i} \longrightarrow E_{i}^{(n)}$ e $\iota_{n}: G_{n} \longrightarrow G$ as projeções e inclusões canônicas, respectivamente. Como $\mathcal{G}$ é um hiper-ideal, temos $\iota_{n} \circ A_{n} \circ\left(\pi_{1 n}, \ldots, \pi_{m n}\right) \in \mathcal{G}\left(E_{1}, \ldots, E_{m} ; G\right)$. Agora

$$
\begin{aligned}
\sum_{n=1}^{\infty}\left\|\iota_{n} \circ A_{n} \circ\left(\pi_{1 n}, \ldots, \pi_{m n}\right)\right\|_{\mathcal{G}}^{p_{m}} & \leq \sum_{n=1}^{\infty}\left\|\iota_{n}\right\|^{p_{m}} \cdot\left\|A_{n}\right\|_{\mathcal{G}}^{p_{m}} \cdot\left\|\pi_{1 n}\right\|^{p_{m}} \cdots\left\|\pi_{m n}\right\|^{p_{m}} \\
& =\sum_{n=1}^{\infty} \frac{1}{2^{n}}=1<\infty
\end{aligned}
$$

e $\left(\mathcal{G},\|\cdot\|_{\mathcal{G}}\right)$ é hiper-ideal $\left(p_{n}\right)_{n=1}^{\infty}$-Banach. Então, pelo Critério da Série (Teorema 2.1.9) e pela hipótese, temos

$$
A:=\sum_{n=1}^{\infty} \iota_{n} \circ A_{n} \circ\left(\pi_{1 n}, \ldots, \pi_{m n}\right) \in \mathcal{G}\left(E_{1}, \ldots, E_{m} ; G\right) \subseteq \mathcal{H}\left(E_{1}, \ldots, E_{m} ; G\right) .
$$

Por outro lado, chamando de $\iota_{\text {in }}: E_{i}^{(n)} \longrightarrow E_{i}, i=1, \ldots, m$, e $\pi_{n}: G \longrightarrow G_{n}$ as inclusões e projeções usuais, temos $A_{n}=\pi_{n} \circ A \circ\left(\iota_{1 n}, \ldots, \iota_{m n}\right)$ e

$$
n \leq\left\|A_{n}\right\|_{\mathcal{H}}=\left\|\pi_{n} \circ A \circ\left(\iota_{1 n}, \ldots, \iota_{m n}\right)\right\|_{\mathcal{H}} \leq\left\|\pi_{n}\right\| \cdot\|A\|_{\mathcal{H}} \cdot\left\|\iota_{1 n}\right\| \cdots\left\|\iota_{m n}\right\|,
$$

contradição esta que completa a demonstração. 


\section{$2.2 \quad$ Exemplos}

Nesta seção apresentaremos importantes exemplos de hiper-ideais, por exemplo exibiremos o menor hiper-ideal e o menor hiper-ideal de Banach; e marcaremos a diferença com a teoria de multi-ideais mostrando que alguns multi-ideais clássicos não são hiper-ideais.

No que se segue, a menos de menção explícita em contrário, estaremos considerando $\|\cdot\|$ como sendo a norma uniforme restrita à classe $\mathcal{H}$ em questão.

Exemplo 2.2.1. O objetivo deste exemplo é mostrar que, diferentemente do caso de multi-ideais, a classe $\mathcal{L}_{f}$ das aplicaões multilineares de tipo finito não é um hiper-ideal. Para isso considere a aplicação bilinear contínua

$$
T: \ell_{2} \times \ell_{2} \longrightarrow \mathbb{K}, T(x, y)=\sum_{i=1}^{\infty} x_{i} y_{i} .
$$

Vejamos que $T$ não é de tipo finito. Caso fosse, poderíamos escrever

$$
T(x, y)=\sum_{j=1}^{k} \varphi_{j}^{(1)}(x) \varphi_{j}^{(2)}(y)
$$

para todos $x, y \in \ell_{2}$, onde $\varphi_{j}^{(1)}, \varphi_{j}^{(2)} \in \ell_{2}^{\prime}$ para $j=1, \ldots, k$. Da dualidade $\ell_{2}^{\prime}=\ell_{2}$, para cada $j=1, \ldots, k$, existem $\left(a_{i}^{(j)}\right)_{i=1}^{\infty},\left(b_{i}^{(j)}\right)_{i=1}^{\infty} \in \ell_{2}$ tais que

$$
\varphi_{j}^{(1)}(x)=\sum_{i=1}^{\infty} a_{i}^{(j)} x_{i} \text { e } \varphi_{j}^{(2)}(y)=\sum_{i=1}^{\infty} b_{i}^{(j)} y_{i}
$$

para todos $x=\left(x_{i}\right)_{i=1}^{\infty}$ e $y=\left(y_{i}\right)_{i=1}^{\infty}$ em $\ell_{2}$. Dessa forma,

$$
T(x, y)=\sum_{j=1}^{k}\left[\left(\sum_{i=1}^{\infty} a_{i}^{(j)} x_{i}\right) \cdot\left(\sum_{i=1}^{\infty} b_{i}^{(j)} y_{i}\right)\right]
$$

para todos $x, y \in \ell_{2}$. Mas, pela definição de $T$, para cada $i$ tomando o $i$-ésimo vetor unitário canônico $e_{i}$, tem-se

$$
1=T\left(e_{i}, e_{i}\right)=\sum_{j=1}^{k} a_{i}^{(j)} b_{i}^{(j)} .
$$

Note que o último termo da igualdade acima tende a zero quando $i \longrightarrow \infty$, pois trata-se de uma soma finita de produtos de termos gerais de séries absolutamente convergentes. Essa contradição mostra que $T$ não é de tipo finito.

Como, segundo a Observação 2.1.3(iii), um hiper-ideal deve conter todas as formas $n$-lineares contínuas, concluímos que $\mathcal{L}_{f}$ não é um hiper-ideal. 
Na teoria de multi-ideais, $\mathcal{L}_{f}$ tem o papel destacado de ser o menor multi-ideal. Como $\mathcal{L}_{f}$ não é hiper-ideal, precisamos de um hiper-ideal que desempenhe este mesmo papel na teoria de hiper-ideais. Em particular, este hiper-ideal deve coincidir, no caso linear, com os operadores lineares de tipo/posto finito. É natural então pensar na classe das aplicações multilineares de posto finito:

Teorema 2.2.2. A classe $\mathcal{L}_{\mathcal{F}}$ das aplicações multilineares de posto finito, munida da norma uniforme, forma o menor hiper-ideal normado. Isso significa que se $\left(\mathcal{H},\|\cdot\|_{\mathcal{H}}\right)$ é um hiper-ideal normado, então $\mathcal{L}_{\mathcal{F}} \subseteq \mathcal{H}$.

Demonstração. Como a condição (1) da Definição 2.1.2 é a mesma de multi-ideais e já sabemos que $\mathcal{L}_{\mathcal{F}}$ é multi-ideal, não há o que fazer em relação a esta condição.

Verifiquemos a condição (2). Para isso sejam $1 \leq m_{1}<\cdots<m_{n}$ números naturais, $A \in \mathcal{L}_{\mathcal{F}}\left(E_{1}, \ldots, E_{n} ; F\right), B_{1} \in \mathcal{L}\left(G_{1}, \ldots, G_{m_{1}} ; E_{1}\right), \ldots, B_{n} \in \mathcal{L}\left(G_{m_{n-1}+1}, \ldots, G_{m_{n}} ; E_{n}\right) \mathrm{e}$ $t \in \mathcal{L}(F ; H)$. Sendo $A$ de posto finito, podemos escrever $A=\sum_{j=1}^{k} T_{j} \otimes y_{j}$, onde cada $T_{j} \in \mathcal{L}\left(E_{1}, \ldots, E_{n}\right)$ e cada $y_{j} \in F$. Então

$$
\begin{aligned}
t \circ A \circ\left(B_{1}, \ldots, B_{n}\right) & \left(x_{1}, \ldots, x_{m_{n}}\right) \\
& =t\left(A\left(B_{1}\left(x_{1}, \ldots, x_{m_{1}}\right), \ldots, B_{n}\left(x_{m_{n-1}+1}, \ldots, x_{m_{n}}\right)\right)\right) \\
& =t\left(\sum_{j=1}^{k} T_{j}\left(B_{1}\left(x_{1}, \ldots, x_{m_{1}}\right), \ldots, B_{n}\left(x_{m_{n-1}+1}, \ldots, x_{m_{n}}\right)\right) y_{j}\right) \\
& =\sum_{j=1}^{k} T_{j}\left(B_{1}\left(x_{1}, \ldots, x_{m_{1}}\right), \ldots, B_{n}\left(x_{m_{n-1}+1}, \ldots, x_{m_{n}}\right)\right) t\left(y_{j}\right) \\
& =\sum_{j=1}^{k} S_{j} \otimes z_{j}\left(x_{1}, \ldots, x_{m_{n}}\right)
\end{aligned}
$$

para todos $x_{i} \in G_{i}$, onde $S_{j}:=T_{j} \circ\left(B_{1}, \ldots, B_{n}\right) \in \mathcal{L}\left(G_{1}, \ldots, G_{m_{n}}\right)$ e $z_{j}=t\left(y_{j}\right) \in H$. Logo $t \circ A \circ\left(B_{1}, \ldots, B_{n}\right) \in \mathcal{L}_{\mathcal{F}}\left(G_{1}, \ldots, G_{m_{n}} ; H\right)$, provando que $\mathcal{L}_{\mathcal{F}}$ é hiper-ideal.

Mostremos agora que $\mathcal{L}_{\mathcal{F}}$ é o menor hiper-ideal. Para isso sejam $\mathcal{H}$ um hiper-ideal e $A \in \mathcal{L}_{\mathcal{F}}\left(E_{1}, \ldots, E_{n} ; F\right)$. Devemos provar que $A \in \mathcal{H}\left(E_{1}, \ldots, E_{n} ; F\right)$. Podemos supor, sem perda de generalidade, que

$$
A=T \otimes y
$$

onde $T \in \mathcal{L}\left(E_{1}, \ldots, E_{n}\right)$ e $y \in F$, visto que $A$ pode ser escrito na forma $\sum_{j=1}^{k} T_{j} \otimes y_{j}$, onde $T_{j} \in \mathcal{L}\left(E_{1}, \ldots, E_{n}\right)$ e $y_{j} \in F$ e $\mathcal{H}\left(E_{1}, \ldots, E_{n} ; F\right)$ é um subespaço vetorial de 
$\mathcal{L}\left(E_{1}, \ldots, E_{n} ; F\right)$. Consideremos o operador linear

$$
1 \otimes y: \mathbb{K} \longrightarrow F, 1 \otimes y(\lambda)=\lambda y
$$

Temos $1 \otimes y \in \mathcal{L}_{f}(\mathbb{K} ; F) \subseteq \mathcal{H}(\mathbb{K} ; F)$. E da propriedade de hiper-ideal segue que

$$
A=T \otimes y=(1 \otimes y) \circ T \in \mathcal{H}\left(E_{1}, \ldots, E_{n} ; F\right) .
$$

Conforme anunciado na Observação 2.1.3(iii), em um hiper-ideal, conter as aplicações multilineares de tipo finito é equivalente a uma condição aparentemente mais exigente, a saber, conter as aplicações multilineares de posto finito. De fato, da proposição anterior segue o:

Corolário 2.2.3. Seja $\mathcal{H}$ uma subclasse da classe das aplicações multilineares contínuas entre espaços de Banach que satisfaz a propriedade de hiper-ideal. Para todo $n \in \mathbb{N} e$ quaisquer espaços de Banach $E_{1}, \ldots, E_{n}, F$, são equivalentes:

(1) $\mathcal{H}\left(E_{1}, \ldots, E_{n} ; F\right)$ é um subespaço vetorial de $\mathcal{L}\left(E_{1}, \ldots, E_{n} ; F\right)$ que contém as aplicações n-lineares de tipo finito;

$\left(1^{\prime}\right) \mathcal{H}\left(E_{1}, \ldots, E_{n} ; F\right)$ é um subespaço vetorial de $\mathcal{L}\left(E_{1}, \ldots, E_{n} ; F\right)$ que contém as aplicações n-lineares de posto finito.

Como a classe das aplicações de posto finito é um hiper-ideal, pela Proposição 2.1.5 sabemos que a classe $\overline{\mathcal{L}_{\mathcal{F}}}$ das aplicações aproximáveis por aplicações de posto finito é um hiper-ideal de Banach com a norma uniforme. Já sabemos também que a classe $\mathcal{L}_{f}$ das aplicações de tipo finito não é um hiper-ideal. A questão é saber se a classe $\overline{\mathcal{L}_{f}}$ das aplicações aproximáveis por aplicações de tipo finito é um hiper-ideal ou não.

Exemplo 2.2.4. Vejamos que a classe $\overline{\mathcal{L}_{f}}$ das aplicações aproximáveis por aplicações de tipo finito não é um hiper-ideal.

Para isso seja $T: \ell_{2} \times \ell_{2} \longrightarrow \mathbb{K}$ a aplicação bilinear contínua do Exemplo 2.2.1, a qual já sabemos não ser de tipo finito. Afirmamos que $T$ não é aproximável por aplicações de tipo finito, isto é, existe $\varepsilon>0$ tal que $\|T-S\|>\varepsilon$ para todo $S \in \mathcal{L}_{f}\left({ }^{2} \ell_{2}\right)$.

De fato, consideremos $0<\varepsilon<1$ e suponhamos que exista $S \in \mathcal{L}_{f}\left({ }^{2} \ell_{2}\right)$ de forma que $\|T-S\|<\varepsilon$. Digamos

$$
S(x, y)=\sum_{j=1}^{k}\left[\left(\sum_{i=1}^{\infty} a_{i}^{(j)} x_{i}\right) \cdot\left(\sum_{i=1}^{\infty} b_{i}^{(j)} y_{i}\right)\right]
$$


onde $\left(a_{i}^{(j)}\right)_{i=1}^{\infty},\left(b_{i}^{(j)}\right)_{i=1}^{\infty} \in \ell_{2}, j=1, \ldots, k$. Daí,

$$
\left|1-\sum_{j=1}^{k} a_{i}^{(j)} b_{i}^{(j)}\right|=\left|T\left(e_{i}, e_{i}\right)-S\left(e_{i}, e_{i}\right)\right| \leq\|T-S\|<\varepsilon .
$$

Fazendo $i \longrightarrow \infty$, obtemos $1 \leq \varepsilon$, um absurdo. Segue da Observação 2.1.3(iii) que a classe $\overline{\mathcal{L}_{f}}$ das aplicações aproximáveis por aplicações de tipo finito não é hiper-ideal.

Definição 2.2.5. ([27, Definition 1.26]) Uma aplicação $n$-linear $A \in \mathcal{L}\left(E_{1}, \ldots, E_{n} ; F\right)$ é dita nuclear se podemos encontrar, para cada $l=1, \ldots, n$, uma sequência limitada $\left(\varphi_{j}^{(l)}\right)_{j=1}^{\infty}$ de funcionais lineares em $E_{l}^{\prime}$, uma sequência $\left(\lambda_{j}\right)_{j=1}^{\infty} \in \ell_{1}$ e $\left(y_{j}\right)_{j=1}^{\infty}$ uma sequência limitada em $F$ tais que

$$
A\left(x_{1}, \ldots, x_{n}\right)=\sum_{j=1}^{\infty} \lambda_{j} \varphi_{j}^{(1)}\left(x_{1}\right) \cdots \varphi_{j}^{(n)}\left(x_{n}\right) \cdot y_{j}
$$

para todos $x_{1} \in E_{1}, \ldots, x_{n} \in E_{n}$. Nesse caso chamamos a expressão acima de representação nuclear de $A$ e escrevemos $A \in \mathcal{L}_{\mathcal{N}}\left(E_{1}, \ldots, E_{n} ; F\right)$. Definimos ainda a função $\|\cdot\|_{\mathcal{L}_{\mathcal{N}}}: \mathcal{L}_{\mathcal{N}} \longrightarrow[0, \infty)$ por

$$
\|A\|_{\mathcal{L}_{\mathcal{N}}}=\inf \left\{\sum_{j=1}^{\infty}\left|\lambda_{j}\right| \cdot\left\|\varphi_{j}^{(1)}\right\| \cdots\left\|\varphi_{j}^{(n)}\right\| \cdot\left\|y_{j}\right\|\right\},
$$

onde o ínfimo é tomado sobre todas as representações nucleares de $A$.

Exemplo 2.2.6. Vejamos que a classe das aplicações multilineares nucleares não forma um hiper-ideal. Primeiramente, notemos que a classe das aplicações nucleares está contida na classe das aplicações multilineares aproximáveis por aplicações de tipo finito. De fato, se $A \in \mathcal{L}_{\mathcal{N}}\left(E_{1}, \ldots, E_{n} ; F\right)$ então podemos considerar sequências $\left(\varphi_{j}^{(1)}\right)_{j=1}^{\infty}$ em $E_{l}^{\prime}$, $l=1, \ldots, n,\left(\lambda_{j}\right)_{j=1}^{\infty} \in \ell_{1}$ e $\left(y_{j}\right)_{j=1}^{\infty}$ em $F$ de modo a obtermos uma representação nuclear de $A$. Daí podemos definir $\left(A_{k}\right)_{k=1}^{\infty}$ em $\mathcal{L}_{f}\left(E_{1}, \ldots, E_{n} ; F\right)$ onde cada $A_{k}$ é dada por

$$
A_{k}\left(x_{1}, \ldots, x_{n}\right)=\sum_{j=1}^{k} \lambda_{j} \varphi_{j}^{(1)}\left(x_{1}\right) \cdots \varphi_{j}^{(n)}\left(x_{n}\right) \cdot y_{j}
$$

para todos $x_{1} \in E_{1}, \ldots, x_{n} \in E_{n}$. Com isso, a série $\sum_{j=1}^{\infty}\left|\lambda_{j}\right| \cdot\left\|\varphi_{1}^{(j)}\right\| \cdots\left\|\varphi_{n}^{(j)}\right\| \cdot\left\|y_{j}\right\|$ é convergente, e portanto, dado $\varepsilon>0$ podemos encontrar $k_{0}$ tal que

$$
\sum_{j=k_{0}}^{\infty}\left|\lambda_{j}\right| \cdot\left\|\varphi_{j}^{(1)}\right\| \cdots\left\|\varphi_{j}^{(n)}\right\| \cdot\left\|y_{j}\right\|<\varepsilon .
$$


Assim

$$
\left\|A_{k}-A\right\| \leq \sum_{j=k_{0}}^{\infty}\left|\lambda_{j}\right| \cdot\left\|\varphi_{j}^{(1)}\right\| \cdots\left\|\varphi_{j}^{(n)}\right\| \cdot\left\|y_{j}\right\|<\varepsilon
$$

para $k \geq k_{0}$. Portanto $A$ é aproximável por aplicações de tipo finito.

A demonstração de que $\mathcal{L}_{\mathcal{N}}$ é um multi-ideal pode ser encontrada em [35, Proposition 2.2]. Em particular, $\|\cdot\| \leq\|\cdot\|_{\mathcal{L}_{\mathcal{N}}}$.

Podemos agora concluir que a classe das aplicações nucleares não é um hiper-ideal. Para isso basta observar que a classe das aplicações multilineares aproximáveis por aplicações de tipo finito não contém as aplicações de posto finito. Então a classe das aplicações nucleares também não pode contê-la, ou seja, não satisfaz a condição $\left(1^{\prime}\right)$.

É conhecido que a classe das aplicações multilineares nucleares, munida da norma nuclear, forma o menor multi-ideal de Banach. Do exemplo acima sabemos que esta classe não é um hiper-ideal. Devemos então buscar uma classe que desempenhe este mesmo papel na teoria de multi-ideais, ou seja, uma classe que seja o menor hiper-ideal de Banach. De preferência, esta classe deve coincidir, no caso linear, com os operadores lineares nucleares. A classe das aplicações multilineares de posto finito não serve por não ser completa. Veremos na sequência que a seguinte classe, baseada em uma classe introduzida em [46], satisfaz os requisitos desejados.

Definição 2.2.7. Sejam $s \in(0, \infty)$ e $r \in[1, \infty]$ tais que $1 \leq 1 / s+1 / r$. Uma aplicação $n$-linear contínua $A \in \mathcal{L}\left(E_{1}, \ldots, E_{n} ; F\right)$ é dita hiper- $(s ; r)$-nuclear se existem sequências $\left(\lambda_{j}\right)_{j=1}^{\infty} \in \ell_{s},\left(T_{j}\right)_{j=1}^{\infty} \in \ell_{r}^{w}\left(\mathcal{L}\left(E_{1}, \ldots, E_{n}\right)\right)$ e $\left(y_{j}\right)_{j=1}^{\infty} \in \ell_{\infty}(F)$ tais que

$$
A\left(x_{1}, \ldots, x_{n}\right)=\sum_{j=1}^{\infty} \lambda_{j} T_{j} \otimes y_{j}\left(x_{1}, \ldots, x_{n}\right)=\sum_{j=1}^{\infty} \lambda_{j} T_{j}\left(x_{1}, \ldots, x_{n}\right) y_{j},
$$

para todo $\left(x_{1}, \ldots, x_{n}\right) \in E_{1} \times \cdots \times E_{n}$. Nesse caso escrevemos $A \in \mathcal{L}_{\mathcal{H N}}(s, r)$

Definimos ainda a correspondência $\|\cdot\|_{\mathcal{L}_{\mathcal{H N}}(s, r)}: \mathcal{L}_{\mathcal{H N}(s, r)} \longrightarrow[0, \infty)$ por

$$
\|A\|_{\mathcal{L}_{\mathcal{H N}(s, r)}}=\inf \left\{\left\|\left(\lambda_{j}\right)_{j=1}^{\infty}\right\|_{s} \cdot\left\|\left(T_{j}\right)_{j=1}^{\infty}\right\|_{w, r} \cdot\left\|\left(y_{j}\right)_{j=1}^{\infty}\right\|_{\infty}\right\}
$$

onde o ínfimo é tomado sobre todas as representações de $A$ na forma descrita em (2.1).

Observação 2.2.8. ( $i$ ) Notemos que a série em (2.1) de fato é convergente. Vejamos que, dadas sequências $\left(\lambda_{j}\right)_{j=1}^{\infty} \in \ell_{s},\left(T_{j}\right)_{j=1}^{\infty} \in \ell_{r}^{w}\left(\mathcal{L}\left(E_{1}, \ldots, E_{n}\right)\right),\left(y_{j}\right)_{j=1}^{\infty} \in \ell_{\infty}(F)$ e $\left(x_{1}, \ldots, x_{n}\right) \in E_{1} \times \cdots \times E_{n}$, a série em (2.1) é absolutamente convergente, logo convergente. Para isso considere o operador linear

$$
\psi_{\left(x_{1}, \ldots, x_{n}\right)}: \mathcal{L}\left(E_{1}, \ldots, E_{n}\right) \longrightarrow \mathbb{K}, \psi_{\left(x_{1}, \ldots, x_{n}\right)}(T)=T\left(x_{1}, \ldots, x_{n}\right) .
$$


De

$$
\left|\psi_{\left(x_{1}, \ldots, x_{n}\right)}(T)\right|=\left|T\left(x_{1}, \ldots, x_{n}\right)\right| \leq\|T\| \cdot\left\|x_{1}\right\| \cdots\left\|x_{n}\right\|,
$$

temos $\psi_{\left(x_{1}, \ldots, x_{n}\right)} \in \mathcal{L}\left(E_{1}, \ldots, E_{n}\right)^{\prime}$ e $\left\|\psi_{\left(x_{1}, \ldots, x_{n}\right)}\right\| \leq\left\|x_{1}\right\| \cdots\left\|x_{n}\right\|$. Dessa forma

$$
\frac{\psi_{\left(x_{1}, \ldots, x_{n}\right)}}{\left\|x_{1}\right\| \cdots\left\|x_{n}\right\|} \in B_{\mathcal{L}\left(E_{1}, \ldots, E_{n}\right)^{\prime}}
$$

sempre que $x_{i} \neq 0, i=1, \ldots, n$. Nesse caso, da desigualdade de Hölder segue que

$$
\begin{aligned}
\sum_{j=1}^{\infty}\left\|\lambda_{j} T_{j}\left(x_{1}, \ldots, x_{n}\right) y_{j}\right\| & \leq\left\|\left(y_{j}\right)_{j=1}^{\infty}\right\|_{\infty} \cdot\left(\sum_{j=1}^{\infty}\left|\lambda_{j}\right|^{s}\right)^{1 / s} \cdot\left(\sum_{j=1}^{\infty}\left|T_{j}\left(x_{1}, \ldots, x_{n}\right)\right|^{r}\right)^{1 / r} \\
& =\left\|\left(y_{j}\right)_{j=1}^{\infty}\right\|_{\infty} \cdot\left\|\left(\lambda_{j}\right)_{j=1}^{\infty}\right\|_{s} \cdot\left(\sum_{j=1}^{\infty}\left|\psi_{\left(x_{1}, \ldots, x_{n}\right)}\left(T_{j}\right)\right|^{r}\right)^{1 / r} \\
& \leq\left\|\left(y_{j}\right)_{j=1}^{\infty}\right\|_{\infty} \cdot\left\|\left(\lambda_{j}\right)_{j=1}^{\infty}\right\|_{s} \cdot\left\|\left(T_{j}\right)_{j=1}^{\infty}\right\|_{r, w} \cdot\left\|x_{1}\right\| \cdots\left\|x_{n}\right\|<\infty .
\end{aligned}
$$

(ii) No caso em que $s=1$ e $r=\infty$, temos $\left(\lambda_{j}\right)_{j=1}^{\infty} \in \ell_{1}$ e que a sequência $\left(T_{j}\right)_{j=1}^{\infty}$ é limitada (visto que $\ell_{\infty}^{w}(E)=\ell_{\infty}(E)$ para todo espaço de Banach $E$ ). Desse modo as aplicações hiper- $(1, \infty)$-nucleares são uma generalização das aplicações nucleares vistas no Exemplo 2.2.6 (conforme já mencionado, essa classe mais geral foi baseada em classes introduzidas em [46]). As aplicações hiper- $(1, \infty)$-nucleares serão chamadas simplesmente de aplicações hiper-nucleares e escrevemos $\mathcal{L}_{\mathcal{H N}}$ ao invés de $\mathcal{L}_{\mathcal{H} \mathcal{N}_{(1, \infty)}}$. É claro que, no caso linear, esta classe coincide com os operadores lineares nucleares.

Após alguns exemplos de classes que não são hiper-ideais, é hora de dar mais exemplos de hiper-ideais:

Teorema 2.2.9. A classe $\left(\mathcal{L}_{\mathcal{H N}_{(s, r)}},\|\cdot\|_{\mathcal{L}_{\mathcal{H N}}(s, r)}\right)$ das aplicações multilineares hiper- $(s, r)$ nucleares é um hiper-ideal p-Banach, onde $\frac{1}{p}=\frac{1}{s}+\frac{1}{r}$.

Demonstração. Verificaremos que $\left(\mathcal{L}_{\mathcal{H N}_{(s, r)}},\|\cdot\|_{\mathcal{L}_{\mathcal{H N}}(s, r)}\right)$ é um hiper-ideal p-Banach mostrando que as condições do Critério da Série (Teorema 2.1.9) estão satisfeitas:

(i) É claro que $\left.I_{n} \in \mathcal{L}_{\mathcal{H N}_{(s, r)}}{ }^{n} \mathbb{K}\right)$. Verifiquemos que $\left\|I_{n}\right\|_{\mathcal{L}_{\mathcal{H N}}(s, r)}=1$. Como $I_{n}$ pode ser vista como representação de si mesma, da definição de $\|\cdot\|_{\mathcal{L}_{\mathcal{H N}}(s, r)}$ segue que

$$
\left\|I_{n}\right\|_{\mathcal{L}_{\mathcal{H N}}(s, r)} \leq\left\|I_{n}\right\|=1
$$

Suponhamos que não valha a igualdade. Neste caso existe uma representação $\sum_{j=1}^{\infty} \lambda_{j} \otimes T_{j}$ de $I_{n} \operatorname{com}\left\|\left(\lambda_{j}\right)_{j=1}^{\infty}\right\|_{s} \cdot\left\|\left(T_{j}\right)_{j=1}^{\infty}\right\|_{w, r}<1$. Mas $1=\left|I_{n}(1, \ldots, 1)\right| \leq \sum_{j=1}^{\infty}\left|\lambda_{j}\right| \cdot\left\|T_{j}\right\| \cdot\|1\|^{n} \leq\left\|\left(\lambda_{j}\right)_{j=1}^{\infty}\right\|_{s} \cdot\left\|\left(T_{j}\right)_{j=1}^{\infty}\right\|_{r}=\left\|\left(\lambda_{j}\right)_{j=1}^{\infty}\right\|_{s} \cdot\left\|\left(T_{j}\right)_{j=1}^{\infty}\right\|_{w, r}<1$, 
contradição esta que prova a igualdade desejada. Notemos que a igualdade $\left\|\left(T_{j}\right)_{j=1}^{\infty}\right\|_{r}=$ $\left\|\left(T_{j}\right)_{j=1}^{\infty}\right\|_{w, r}$ vale pois o espaço $\mathcal{L}\left({ }^{n} \mathbb{K}\right)$ é de dimensão finita, portanto $\ell_{r}^{w}\left(\mathcal{L}\left({ }^{n} \mathbb{K}\right)\right)=\ell_{r}\left(\mathcal{L}\left({ }^{n} \mathbb{K}\right)\right)$, com igualdade de normas.

(ii) Consideremos $\left(A_{j}\right)_{j=1}^{\infty} \subseteq \mathcal{L}_{\mathcal{H} \mathcal{N}_{(s, r)}}\left(E_{1}, \ldots, E_{n} ; F\right)$ tal que

$$
\sum_{j=1}^{\infty}\left\|A_{j}\right\|_{\mathcal{L}_{\mathcal{H N}(s, r)}^{p}}^{p}<\infty
$$

Então dado $\varepsilon>0$, para cada $j \in \mathbb{N}$ existem $\left(\lambda_{j k}\right)_{k=1}^{\infty} \in \ell_{s},\left(T_{j k}\right)_{k=1}^{\infty} \in \ell_{r}^{w}\left(\mathcal{L}\left(E_{1}, \ldots, E_{n}\right)\right)$ e $\left(y_{j k}\right)_{k=1}^{\infty} \in \ell_{\infty}(F)$ tais que

$$
A_{j}=\sum_{k=1}^{\infty} \lambda_{j k} T_{j k} \otimes y_{j k} \mathrm{e}\left\|\left(\lambda_{j k}\right)_{k=1}^{\infty}\right\|_{s} \cdot\left\|\left(T_{j k}\right)_{k=1}^{\infty}\right\|_{w, r} \cdot\left\|\left(y_{j k}\right)_{k=1}^{\infty}\right\|_{\infty}<(1+\varepsilon)\left\|A_{j}\right\|_{\mathcal{L}_{\mathcal{H N}(s, r)}} .
$$

Sem perda de generalidade podemos supor, para cada $j$, que $\left\|\left(y_{j k}\right)_{k=1}^{\infty}\right\|_{\infty}=1 \mathrm{e}$

$$
\left\|\left(\lambda_{j k}\right)_{k=1}^{\infty}\right\|_{s}<\left((1+\varepsilon)\left\|A_{j}\right\|_{\mathcal{L}_{\mathcal{H N}}(s, r)}\right)^{p / s},\left\|\left(T_{j k}\right)_{k=1}^{\infty}\right\|_{w, r}<\left((1+\varepsilon)\left\|A_{j}\right\|_{\mathcal{L}_{\mathcal{H N}}(s, r)}\right)^{p / r}
$$

pois $\frac{1}{p}=\frac{1}{s}+\frac{1}{r}$. Com isso,

$$
\sum_{j, k=1}^{\infty}\left|\lambda_{j k}\right|^{s}=\sum_{j=1}^{\infty} \sum_{k=1}^{\infty}\left|\lambda_{j k}\right|^{s}=\sum_{j=1}^{\infty}\left\|\left(\lambda_{j k}\right)_{k=1}^{\infty}\right\|_{s}^{s}<(1+\varepsilon)^{p} \cdot \sum_{j=1}^{\infty}\left\|A_{j}\right\|_{\mathcal{L}_{\mathcal{H N}}(s, r)}^{p}<\infty
$$

onde a primeira igualdade é válida por se tratar de uma série de números positivos. Portanto $\left(\lambda_{j k}\right)_{j, k=1}^{\infty} \in \ell_{s}$.

Para todo funcional linear não nulo $\varphi \in\left(\mathcal{L}\left(E_{1}, \ldots, E_{n}\right)\right)^{\prime}$ vale que

$$
\begin{aligned}
\sum_{j, k=1}^{\infty}\left|\varphi\left(T_{j k}\right)\right|^{r} & =\sum_{j=1}^{\infty} \sum_{k=1}^{\infty}\left|\varphi\left(T_{j k}\right)\right|^{r}=\|\varphi\| \cdot\left(\sum_{j=1}^{\infty} \sum_{k=1}^{\infty}\left|\frac{\varphi}{\|\varphi\|}\left(T_{j k}\right)\right|^{r}\right) \\
& \leq\|\varphi\| \cdot\left(\sum_{j=1}^{\infty}\left\|\left(T_{j k}\right)_{k=1}^{\infty}\right\|_{w, r}^{r}\right)<(1+\varepsilon)^{p} \cdot \sum_{j=1}^{\infty}\left\|A_{j}\right\|_{\mathcal{L}_{\mathcal{H N}}(s, r)}<\infty
\end{aligned}
$$

Portanto $\left(T_{j k}\right)_{j, k=1}^{\infty} \in \ell_{r}^{w}\left(\mathcal{L}\left(E_{1}, \ldots, E_{n}\right)\right)$.

Como $\left\|\left(y_{j k}\right)_{k=1}^{\infty}\right\|_{\infty}=1$ para todo $j$, é claro que $\left(y_{j k}\right)_{j, k=1}^{\infty} \in \ell_{\infty}(F)$ e $\left\|\left(y_{j k}\right)_{j, k=1}^{\infty}\right\|_{\infty}=1$.

Vimos na Observação 2.2.8(i) que, nessas condições, para todos $x_{1} \in E_{1}, \ldots, x_{n} \in E_{n}$, a série

$$
\sum_{j, k=1}^{\infty} \lambda_{j k} T_{j k} \otimes y_{j k}\left(x_{1}, \ldots, x_{n}\right)
$$


converge absolutamente no espaço de Banach F. Então converge incondicionalmente, e portanto

$$
\begin{aligned}
\sum_{j, k=1}^{\infty} \lambda_{j k} T_{j k} \otimes y_{j k}\left(x_{1}, \ldots, x_{n}\right) & =\sum_{j=1}^{\infty} \sum_{k=1}^{\infty} \lambda_{j k} T_{j k} \otimes y_{j k}\left(x_{1}, \ldots, x_{n}\right) \\
& =\sum_{j=1}^{\infty} A_{j}\left(x_{1}, \ldots, x_{n}\right)=A\left(x_{1}, \ldots, x_{n}\right) .
\end{aligned}
$$

Logo (2.4) é uma representação para $A$, o que prova que $A \in \mathcal{L}_{\mathcal{H} \mathcal{N}_{(s, r)}}\left(E_{1}, \ldots, E_{n} ; F\right)$. Além disso, por (2.2) e (2.3) temos

$$
\begin{aligned}
\|A\|_{\mathcal{L}_{\mathcal{H N}}(s, r)} & \leq\left\|\left(\lambda_{j k}\right)_{j, k=1}^{\infty}\right\|_{s}^{p} \cdot\left\|\left(T_{j k}\right)_{j, k=1}^{\infty}\right\|_{w, r}^{p} \cdot\left\|\left(y_{j k}\right)_{j, k=1}^{\infty}\right\|_{\infty}^{p} \\
& \leq\left((1+\varepsilon)^{p} \cdot \sum_{j=1}^{\infty}\left\|A_{j}\right\|_{\mathcal{L}_{\mathcal{H N}}(s, r)}^{p}\right)^{p / s} \cdot\left((1+\varepsilon)^{p} \cdot \sum_{j=1}^{\infty}\left\|A_{j}\right\|_{\mathcal{L}_{\mathcal{H \mathcal { N }}}(s, r)}^{p}\right)^{p / r} \\
& =(1+\varepsilon)^{p} \cdot \sum_{j=1}^{\infty}\left\|A_{j}\right\|_{\mathcal{L}_{\mathcal{H \mathcal { N }}}(s, r)}^{p} .
\end{aligned}
$$

Fazendo $\varepsilon \longrightarrow 0$ a desigualdade desejada segue.

(iii) Sejam $1 \leq m_{1}<\cdots<m_{n}, A \in \mathcal{L}_{\mathcal{H N}_{(s, r)}}\left(E_{1}, \ldots, E_{n} ; F\right), B_{1} \in \mathcal{L}\left(G_{1}, \ldots, G_{m_{1}} ; E_{1}\right)$, $\ldots, B_{n} \in \mathcal{L}\left(G_{m_{n-1}+1}, \ldots, G_{m_{n}} ; E_{n}\right)$, e $t \in \mathcal{L}(F ; H)$. Então podemos escrever $A=$ $\sum_{j=1}^{\infty} \lambda_{j} T_{j} \otimes y_{j}, \operatorname{com}\left(\lambda_{j}\right)_{j=1}^{\infty} \in \ell_{s},\left(T_{j}\right)_{j=1}^{\infty} \in \ell_{r}^{w}\left(\mathcal{L}\left(E_{1}, \ldots, E_{n}\right)\right)$ e $\left(y_{j}\right)_{j=1}^{\infty} \in \ell_{\infty}(F)$. Disso segue que

$$
\begin{aligned}
t \circ A \circ( & \left.B_{1}, \ldots, B_{n}\right)\left(x_{1}, \ldots, x_{m_{n}}\right) \\
& =t\left(\sum_{j=1}^{\infty} \lambda_{j} T_{j} \otimes y_{j}\left(B_{1}\left(x_{1}, \ldots, x_{m_{1}}\right), \ldots, B_{n}\left(x_{m_{n-1}+1}, \ldots, x_{m_{n}}\right)\right)\right) \\
& =t\left(\sum_{j=1}^{\infty} \lambda_{j} T_{j}\left(B_{1}\left(x_{1}, \ldots, x_{m_{1}}\right), \ldots, B_{n}\left(x_{m_{n-1}+1}, \ldots, x_{m_{n}}\right)\right) y_{j}\right) \\
& =\sum_{j=1}^{\infty} \lambda_{j} T_{j}\left(B_{1}\left(x_{1}, \ldots, x_{m_{1}}\right), \ldots, B_{n}\left(x_{m_{n-1}+1}, \ldots, x_{m_{n}}\right)\right) t\left(y_{j}\right) \\
& =\sum_{j=1}^{\infty} \lambda_{j} S_{j} \otimes z_{j}\left(x_{1}, \ldots, x_{m_{n}}\right)
\end{aligned}
$$

para todos $x_{1} \in E_{1}, \ldots, x_{n} \in E_{n}$, onde $S_{j}:=T_{j} \circ\left(B_{1}, \ldots, B_{n}\right)$ e $z_{j}=t\left(y_{j}\right)$ para todo $j \in \mathbb{N}$. Como a sequência $\left(y_{j}\right)_{j=1}^{\infty}$ é limitada e o operador linear $t$ é contínuo, $\left(z_{j}\right)_{j=1}^{\infty} \in \ell_{\infty}(H)$ e

$$
\left\|\left(z_{j}\right)_{j=1}^{\infty}\right\|_{\infty} \leq\|t\| \cdot\left\|\left(y_{j}\right)_{j=1}^{\infty}\right\|_{\infty} .
$$


Devemos então provar que $\left(S_{j}\right)_{j=1}^{\infty} \in \ell_{r}^{w}\left(\mathcal{L}\left(G_{1}, \ldots, G_{m_{n}}\right)\right.$. Para isso seja $\psi \in \mathcal{L}\left(G_{1}, \ldots, G_{m_{n}}\right)^{\prime}$. Consideremos o operador linear $L_{\left(B_{1}, \ldots, B_{n}\right)}: \mathcal{L}\left(E_{1}, \ldots, E_{n}\right) \longrightarrow \mathcal{L}\left(G_{1}, \ldots, G_{m_{n}}\right)$ dado por

$$
L_{\left(B_{1}, \ldots, B_{n}\right)}(T)=T \circ\left(B_{1}, \ldots, B_{n}\right)
$$

Claramente $L_{\left(B_{1}, \ldots, B_{n}\right)}$ é contínuo (devido à continuidade das aplicações multilineares $B_{1}, \ldots, B_{n}$, e $\left\|L_{\left(B_{1}, \ldots, B_{n}\right)}\right\| \leq\left\|B_{1}\right\| \cdots\left\|B_{n}\right\|$. Além disso, podemos escrever

$$
\begin{aligned}
\sum_{j=1}^{\infty}\left|\psi\left(S_{j}\right)\right|^{r} & =\sum_{j=1}^{\infty}\left|\psi\left(T_{j} \circ\left(B_{1}, \ldots, B_{n}\right)\right)\right|^{r}=\sum_{j=1}^{\infty}\left|\psi\left(L_{\left(B_{1}, \ldots, B_{n}\right)}\left(T_{j}\right)\right)\right|^{r} \\
& =\sum_{j=1}^{\infty}\left|\psi \circ L_{\left(B_{1}, \ldots, B_{n}\right)}\left(T_{j}\right)\right|^{r} \leq\left\|\psi \circ L_{\left(B_{1}, \ldots, B_{n}\right)}\right\|^{r} \cdot\left\|\left(T_{j}\right)_{j=1}^{\infty}\right\|_{w, r}^{r}<\infty
\end{aligned}
$$

pois $\psi \circ L_{\left(B_{1}, \ldots, B_{n}\right)} \in \mathcal{L}\left(E_{1}, \ldots, E_{n}\right)^{\prime}$ e $\left(T_{j}\right)_{j=1}^{\infty} \in \ell_{r}^{w}\left(\mathcal{L}\left(E_{1}, \ldots, E_{n}\right)\right)$. Sendo $\psi \in \mathcal{L}\left(G_{1}, \ldots, G_{m_{n}}\right)^{\prime}$ arbitrário, concluímos que $\left(S_{j}\right)_{j=1}^{\infty} \in \ell_{r}^{w}\left(\mathcal{L}\left(G_{1}, \ldots, G_{m_{n}}\right)\right)$. Então

$$
\sum_{j=1}^{\infty} \lambda_{j} S_{j} \otimes z_{j}\left(x_{1}, \ldots, x_{m_{n}}\right)
$$

é uma representação de $t \circ A \circ\left(B_{1}, \ldots, B_{n}\right)$ na forma $(2.1)$, e portanto $t \circ A \circ\left(B_{1}, \ldots, B_{n}\right)$ é hiper- $(s, r)$-nuclear. Além disso,

$$
\left\|\left(S_{j}\right)_{j=1}^{\infty}\right\|_{w, r} \leq\left\|L_{\left(B_{1}, \ldots, B_{n}\right)}\right\| \cdot\left\|\left(T_{j}\right)_{j=1}^{\infty}\right\|_{w, r} \leq\left\|B_{1}\right\| \cdots\left\|B_{n}\right\| \cdot\left\|\left(T_{j}\right)_{j=1}^{\infty}\right\|_{w, r} .
$$

Finalmente,

$$
\begin{aligned}
\left\|t \circ A \circ\left(B_{1}, \ldots, B_{n}\right)\right\|_{\mathcal{L}_{\mathcal{H N}}(s, r)} & \leq\left\|\left(\lambda_{j}\right)_{j=1}^{\infty}\right\|_{s} \cdot\left\|\left(S_{j}\right)_{j=1}^{\infty}\right\|_{w, r} \cdot\left\|\left(z_{j}\right)_{j=1}^{\infty}\right\|_{\infty} \\
& \leq\|t\| \cdot\left(\left\|\left(\lambda_{j}\right)_{j=1}^{\infty}\right\|_{s} \cdot\left\|\left(T_{j}\right)_{j=1}^{\infty}\right\|_{w, r} \cdot\left\|\left(y_{j}\right)_{j=1}^{\infty}\right\|_{\infty}\right) \cdot\left\|B_{1}\right\| \cdots\left\|B_{n}\right\| .
\end{aligned}
$$

Tomando o ínfimo sobre todas as representações hiper- $(s, r)$-nucleares de $A$ obtemos

$$
\left\|t \circ A \circ\left(B_{1}, \ldots, B_{n}\right)\right\|_{\mathcal{L}_{\mathcal{H N}}^{(s, r)}} \leq\|t\| \cdot\|A\|_{\mathcal{L}_{\mathcal{H N}}(s, r)} \cdot\left\|B_{1}\right\| \cdots\left\|B_{n}\right\|
$$

completando a demonstração de que $\left(\mathcal{L}_{\mathcal{H N}_{(s, r)},}\|\cdot\|_{\mathcal{L}_{\mathcal{H N}}(s, r)}\right)$ é um hiper-ideal $p$-Banach.

Como caso particular do teorema acima concluímos que a classe $\mathcal{L}_{\mathcal{H N}}$ das aplicações multilineares hiper-nucleares (cf. Observação 2.2.8(ii)) é um hiper-ideal de Banach. Temos ainda $o$

Corolário 2.2.10. $\mathcal{L}_{\mathcal{N}} \varsubsetneqq \mathcal{L}_{\mathcal{H N}}$. 
Demonstração. A inclusão segue das definições. As classes são diferentes pois $\mathcal{L}_{\mathcal{H N}}$ é hiper-ideal (Teorema 2.2.9) e $\mathcal{L}_{\mathcal{N}}$ não é (Exemplo 2.2.6).

Para provar que $\mathcal{L}_{\mathcal{H N}}$ é o menor hiper-ideal de Banach necessitamos da seguinte caracterização da norma hiper-nuclear:

Lema 2.2.11. Para toda aplicação $A \in \mathcal{L}_{\mathcal{H N}}\left(E_{1}, \ldots, E_{n} ; F\right)$, vale que

$$
\|A\|_{\mathcal{L}_{\mathcal{H N}}}=\inf \left\{\sum_{j=1}^{\infty}\left|\lambda_{j}\right| \cdot\left\|T_{j}\right\| \cdot\left\|y_{j}\right\|\right\}
$$

onde o infimo é tomado sobre todas as representações de A na forma (2.1).

Demonstração. De fato, fixemos uma representação $\sum_{j=1}^{\infty} \lambda_{j} T_{j} \otimes y_{j}$ de $A$ na forma (2.1). Temos

$$
\begin{aligned}
\sum_{j=1}^{\infty}\left|\lambda_{j}\right| \cdot\left\|T_{j}\right\| \cdot\left\|y_{j}\right\| & \leq\left\|\left(T_{j}\right)_{j=1}^{\infty}\right\|_{\infty} \cdot\left\|\left(y_{j}\right)_{j=1}^{\infty}\right\|_{\infty} \cdot\left(\sum_{j=1}^{\infty}\left|\lambda_{j}\right|\right) \\
& =\left\|\left(\lambda_{j}\right)_{j=1}^{\infty}\right\|_{1} \cdot\left\|\left(T_{j}\right)_{j=1}^{\infty}\right\|_{\infty} \cdot\left\|\left(y_{j}\right)_{j=1}^{\infty}\right\|_{\infty} .
\end{aligned}
$$

Tomando o ínfimo sobre todas as representações de $A$, temos

$$
\inf \left\{\sum_{j=1}^{\infty}\left|\lambda_{j}\right| \cdot\left\|T_{j}\right\| \cdot\left\|y_{j}\right\|\right\} \leq\|A\|_{\mathcal{L}_{\mathcal{H N}}} .
$$

Por outro lado, podemos supor sem perda de generalidade que $T_{j} \neq 0$ e $y_{j} \neq 0$ para todo $j \in \mathbb{N}$. Consideremos as sequências $\left(\mu_{j}\right)_{j=1}^{\infty} \subseteq \mathbb{K},\left(S_{j}\right)_{j=1}^{\infty} \subseteq \mathcal{L}\left(E_{1}, \ldots, E_{n}\right)$ e $\left(z_{j}\right)_{j=1}^{\infty} \subseteq F$ dadas por:

$$
\mu_{j}=\lambda_{j}\left\|T_{j}\right\| \cdot\left\|y_{j}\right\|, S_{j}=\frac{T_{j}}{\left\|T_{j}\right\|} \text { e } z_{j}=\frac{y_{j}}{\left\|y_{j}\right\|}
$$

Notemos que $\sum_{j=1}^{\infty} \mu_{j} S_{j} \otimes z_{j}$ é uma representação de $A$, pois:

- $\left\|\left(\mu_{j}\right)_{j=1}^{\infty}\right\|_{1}=\sum_{j=1}^{\infty}\left|\mu_{j}\right|=\sum_{j=1}^{\infty}\left|\lambda_{j}\right| \cdot\left\|T_{j}\right\| \cdot\left\|y_{j}\right\| \leq\left\|\left(\lambda_{j}\right)_{j=1}^{\infty}\right\|_{1} \cdot\left\|\left(T_{j}\right)_{j=1}^{\infty}\right\|_{\infty} \cdot\left\|\left(y_{j}\right)_{j=1}^{\infty}\right\|_{\infty}<\infty$;

- $\left\|\left(S_{j}\right)_{j=1}^{\infty}\right\|_{\infty}=\left\|\left(z_{j}\right)_{j=1}^{\infty}\right\|_{\infty}=1$;

- $\sum_{j=1}^{\infty} \mu_{j} S_{j} \otimes z_{j}=\sum_{j=1}^{\infty} \lambda_{j} T_{j} \otimes y_{j}=A$.

Temos então

$$
\left\|\left(\mu_{j}\right)_{j=1}^{\infty}\right\|_{1} \cdot\left\|\left(S_{j}\right)_{j=1}^{\infty}\right\|_{\infty} \cdot\left\|\left(z_{j}\right)_{j=1}^{\infty}\right\|_{\infty}=\sum_{j=1}^{\infty}\left|\lambda_{j}\right| \cdot\left\|T_{j}\right\| \cdot\left\|y_{j}\right\|,
$$


e portanto

$$
\left\{\sum_{j=1}^{\infty}\left|\lambda_{j}\right| \cdot\left\|T_{j}\right\| \cdot\left\|y_{j}\right\|\right\} \subseteq\left\{\left\|\left(\mu_{j}\right)_{j=1}^{\infty}\right\|_{1} \cdot\left\|\left(S_{j}\right)_{j=1}^{\infty}\right\|_{\infty} \cdot\left\|\left(z_{j}\right)_{j=1}^{\infty}\right\|_{\infty}\right\} .
$$

Por fim,

$$
\|A\|_{\mathcal{L}_{\mathcal{H N}}}=\inf \left\{\left\|\left(\mu_{j}\right)_{j=1}^{\infty}\right\|_{1} \cdot\left\|\left(S_{j}\right)_{j=1}^{\infty}\right\|_{\infty} \cdot\left\|\left(z_{j}\right)_{j=1}^{\infty}\right\|_{\infty}\right\} \leq \inf \left\{\sum_{j=1}^{\infty}\left|\lambda_{j}\right| \cdot\left\|T_{j}\right\| \cdot\left\|y_{j}\right\|,\right\}
$$

donde segue a igualdade.

Chegamos, finalmente, ao resultado procurado:

Teorema 2.2.12. A classe $\left(\mathcal{L}_{\mathcal{H N}},\|\cdot\|_{\mathcal{L}_{\mathcal{H N}}}\right)$ das aplicações multilineares hiper-nucleares é o menor hiper-ideal de Banach, no sentido de que $\left(\mathcal{L}_{\mathcal{H N}},\|\cdot\|_{\mathcal{L}_{\mathcal{H N}}}\right)$ é um hiper-ideal de Banach e se $\left(\mathcal{H},\|\cdot\|_{\mathcal{H}}\right)$ é um hiper-ideal de Banach arbitrário, então $\mathcal{L}_{\mathcal{H N}} \subseteq \mathcal{H}$ e $\|\cdot\|_{\mathcal{H}} \leq\|\cdot\|_{\mathcal{L}_{\mathcal{H N}}}$.

Demonstração. Já sabemos do Teorema 2.2 .9 que $\left(\mathcal{L}_{\mathcal{H N}},\|\cdot\|_{\mathcal{L}_{\mathcal{H N}}}\right)$ é um hiper-ideal de Banach. Seja $\left(\mathcal{H},\|\cdot\|_{\mathcal{H}}\right)$ um hiper-ideal de Banach. Dada $A \in \mathcal{L}_{\mathcal{H N}}\left(E_{1}, \ldots, E_{n} ; F\right)$, tome uma representação

$$
\sum_{j=1}^{\infty} \lambda_{j} T_{j} \otimes y_{j}
$$

de $A$ na forma (2.1). Pelo Lema 2.2.11,

$$
\sum_{j=1}^{\infty}\left|\lambda_{j}\right| \cdot\left\|T_{j}\right\| \cdot\left\|y_{j}\right\|<\infty
$$

Então, dado $\varepsilon>0$ existe $k_{0} \in \mathbb{N}$ tal que, para $k \geq k_{0}$,

$$
\sum_{j=k}^{\infty}\left|\lambda_{j}\right| \cdot\left\|T_{j}\right\| \cdot\left\|y_{j}\right\|<\varepsilon
$$

Por outro lado, consideremos $B_{k}:=\sum_{j=1}^{k} \lambda_{j} T_{j} \otimes y_{j}$. Como $\mathcal{H}$ é um hiper-ideal e cada $B_{k}$ tem posto finito, pela Proposição 2.2.2 temos $B_{k} \in \mathcal{H}\left(E_{1}, \ldots, E_{n} ; F\right)$. Além disso, para todos $k>i \geq k_{0}$,

$$
\left\|B_{k}-B_{i}\right\|_{\mathcal{H}}=\left\|\sum_{j=i+1}^{k} \lambda_{j} T_{j} \otimes y_{j}\right\|_{\mathcal{H}} \leq \sum_{j=i+1}^{k}\left|\lambda_{j}\right| \cdot\left\|T_{j} \otimes y_{j}\right\|_{\mathcal{H}}=\sum_{j=i+1}^{k}\left|\lambda_{j}\right| \cdot\left\|T_{j}\right\| \cdot\left\|y_{j}\right\|<\varepsilon,
$$


ou seja, $\left(B_{k}\right)_{k=1}^{\infty}$ é uma sequência de Cauchy. Sendo $\left(\mathcal{H},\|\cdot\|_{\mathcal{H}}\right)$ um hiper-ideal de Banach, $\left(B_{k}\right)_{k=1}^{\infty}$ converge para alguma aplicação $n$-linear $B \in \mathcal{H}\left(E_{1}, \ldots, E_{n} ; F\right)$ na norma $\|\cdot\|_{\mathcal{H}}$. Da desigualdade $\|\cdot\| \leq\|\cdot\|_{\mathcal{H}}$ (cf. Proposição 2.1.6), sabemos que a convergencia na norma $\|\cdot\|_{\mathcal{H}}$ implica na convergência na norma uniforme; e na norma uniforme $\left(B_{k}\right)_{k=1}^{\infty}$ converge para $A$, então $A=B$. Portanto $A \in \mathcal{H}\left(E_{1}, \ldots, E_{n} ; F\right)$ e $\mathcal{L}_{\mathcal{H N}} \subseteq \mathcal{H}$ como queríamos.

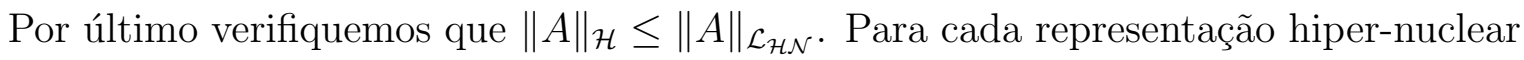
$\sum_{j=1}^{\infty} \lambda_{j} T_{j} \otimes y_{j}$ de $A$, temos

$$
\|A\|_{\mathcal{H}} \leq \sum_{j=1}^{\infty}\left\|\lambda_{j} T_{j} \otimes y_{j}\right\|_{\mathcal{H}}=\sum_{j=1}^{\infty}\left\|\lambda_{j} T_{j} \otimes y_{j}\right\|,
$$

onde a última igualdade segue da Proposição 2.1.7. Tomando o ínfimo sobre todas as representações hiper-nucleares de $A$ segue do Lema 2.2 .11 que $\|A\|_{\mathcal{H}} \leq\|A\|_{\mathcal{L}_{\mathcal{H N}}}$.

Corolário 2.2.13. Valem as seguintes inclusões:

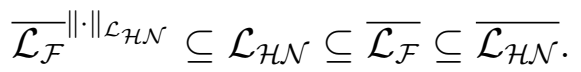

Demonstração. De fato, por ser un hiper-ideal de Banach sabemos que cada componente de $\left(\mathcal{L}_{\mathcal{H N}},\|\cdot\|_{\mathcal{L}_{\mathcal{H N}}}\right)$ é um espaço de Banach que contém as aplicações de posto finito. Então, de $\mathcal{L}_{\mathcal{F}} \subseteq \mathcal{L}_{\mathcal{H N}}$, obtemos $\overline{\overline{\mathcal{L}}_{\mathcal{F}}}\|\cdot\|_{\mathcal{L}_{\mathcal{H N}}} \subseteq \overline{\mathcal{L}_{\mathcal{H N}}}\|\cdot\|_{\mathcal{L}_{\mathcal{H N}}}=\mathcal{L}_{\mathcal{H N}}$ e $\overline{\mathcal{L}_{\mathcal{F}}} \subseteq \overline{\mathcal{L}_{\mathcal{H N}}}$

Agora, dada $A \in \mathcal{L}_{\mathcal{H N}}\left(E_{1}, \ldots, E_{n} ; F\right)$ com representação hiper-nuclear $\sum_{j=1}^{\infty} \lambda_{j} T_{j} \otimes y_{j}$, para cada $k$ considere

$$
B_{k}:=\sum_{j=1}^{k} \lambda_{j} T_{j} \otimes y_{j} \in \mathcal{L}_{\mathcal{F}}\left(E_{1}, \ldots, E_{n} ; F\right) .
$$

Então dado $\varepsilon>0$, para $k$ suficientemente grande tem-se

$$
\left\|A-B_{k}\right\| \leq \sum_{j=k+1}^{\infty}\left|\lambda_{j}\right| \cdot\left\|T_{j}\right\| \cdot\left\|y_{j}\right\|<\varepsilon,
$$

ou seja, $\left(B_{k}\right)_{k=1}^{\infty}$ converge para $A$ na norma uniforme, o que implica $A \in \overline{\mathcal{L}_{\mathcal{F}}}\left(E_{1}, \ldots, E_{n} ; F\right)$.

Observação 2.2.14. Na linha do que foi feito nesta seção, verificaremos mais adiante (cf. Exemplo 2.4.3) que um outro multi-ideal muito estudado, a saber a classe das aplicações multilineares fracamente sequencialmente contínuas, não forma um hiper-ideal. Também neste caso apresentaremos um novo hiper-ideal que, assim como as aplicações fracamente sequencialmente contínuas, coincide no caso linear com os operadores completamente contínuos (cf. Proposição 2.4.7). 


\subsection{Método da fatoração}

Buscamos a partir de agora maneiras de construir hiper-ideais a partir de subclasses da classe das aplicações multilineares contínuas entre espaços de Banach. O primeiro passo é, naturalmente, tentar generalizar os métodos descritos na Seção 1.5 para multi-ideais. Nesta seção tentamos generalizar o método da fatoração. As dificuldades encontradas nesta tentativa devem ser entendidas como manifestações de como é restritiva a condição de hiper-ideal. Explorando essas dificuldades, e buscando alternativas, estamos testando os limites do conceito de hiper-ideal.

Definição 2.3.1. Seja $n \in \mathbb{N}$. Uma partição do conjunto $\{1, \ldots, n\}$ é uma coleção de subconjuntos dois a dois disjuntos da seguinte forma:

$$
\left\{j_{1}^{(1)}, \ldots, j_{k_{1}}^{(1)}\right\} \cup\left\{j_{1}^{(2)}, \ldots, j_{k_{2}}^{(2)}\right\} \cup \cdots \cup\left\{j_{1}^{(m)}, \ldots, j_{k_{m}}^{(m)}\right\}=\{1, \ldots, n\},
$$

onde $m, k_{1}, \ldots, k_{m} \in \mathbb{N}, n=k_{1}+\cdots+k_{m}, j_{1}^{(1)}=1, j_{k_{m}}^{(m)}=n, j_{1}^{(i)}<\cdots<j_{k_{i}}^{(i)} \mathrm{e}$ $j_{k_{i}}^{(i)}<j_{k_{i+1}}^{(i+1)}, i=1, \ldots, m-1$. Nesse caso escrevemos simplesmente que $\left\{j_{1}^{(1)}, \ldots, j_{k_{1}}^{(1)}\right\} \cup$ $\cdots \cup\left\{j_{1}^{(m)}, \ldots, j_{k_{m}}^{(m)}\right\}$ é uma partição de $\{1, \ldots, n\}$. Dizemos ainda que uma partição como esta tem comprimento $m$.

Por exemplo, $\{1,3\} \cup\{2,4\}$ é um partição de $\{1,2,3,4\}$ de comprimento 2 .

As duas técnicas a seguir são tentativas a priori legítimas de método da fatoração para hiper-ideais:

Definição 2.3.2 (Métodos da fatoração fraco e forte). Seja $\mathcal{G}$ uma subclasse da classe das aplicações multilineares contínuas entre espaços de Banach. Denotamos por $\mathcal{G}^{n}$ a união de todas as componentes $\mathcal{G}\left(E_{1}, \ldots, E_{n} ; F\right):=\mathcal{G} \cap \mathcal{L}\left(E_{1}, \ldots, E_{n} ; F\right)$, onde $E_{1}, \ldots, E_{n}$ e $F$ são espaços de Banach. Suponhamos que $\mathcal{G}^{1}$ seja um ideal de operadores.

(i) Dizemos que uma aplicação $n$-linear $A \in \mathcal{L}\left(E_{1}, \ldots, E_{n} ; F\right)$ pertence a $\mathcal{H}_{w}(\mathcal{G})$, e neste caso escrevemos $A \in \mathcal{H}_{w}(\mathcal{G})\left(E_{1}, \ldots, E_{n} ; F\right)$, se existem uma partição $\Pi=\left\{j_{1}^{(1)}, \ldots, j_{k_{1}}^{(1)}\right\} \cup$ $\cdots \cup\left\{j_{1}^{(m)}, \ldots, j_{k_{m}}^{(m)}\right\}$ de $\{1, \ldots, n\}$, espaços de Banach $G_{1}, \ldots, G_{m}$ e aplicações $B_{i} \in$ $\mathcal{G}\left(E_{j_{1}^{(i)}}, \ldots, E_{j_{k_{i}}(i)} ; G_{i}\right), i=1, \ldots, m$ e $C \in \mathcal{L}\left(G_{1}, \ldots, G_{m} ; F\right)$ tais que

$$
A=C \circ\left(B_{1}, \ldots, B_{m}\right)
$$

Neste caso dizemos que $C \circ\left(B_{1}, \ldots, B_{m}\right)$ é uma fatoração de $A$ por $\mathcal{G}$ em relação à partição $\Pi$.

(ii) No caso em que $A$ se fatora por $\mathcal{G}$ em relação a qualquer partição de $\{1, \ldots, n\}$, 
dizemos que $A$ pertence a $\mathcal{H}_{s}(\mathcal{G})$ e escrevemos $A \in \mathcal{H}_{s}(\mathcal{G})\left(E_{1}, \ldots, E_{n} ; F\right)$.

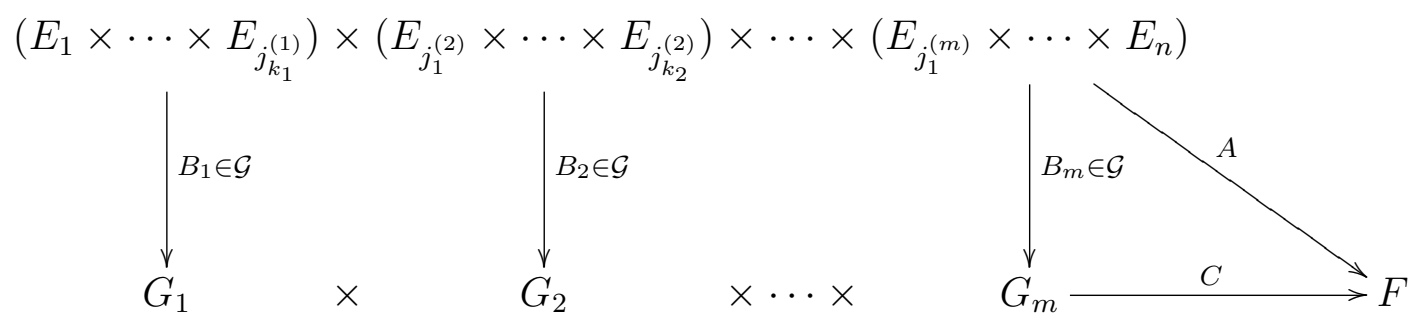

Mimetizando a quasi-norma do método da fatoração para multi-ideais, definimos a função $\|\cdot\|_{\mathcal{H}_{w}(\mathcal{G})}: \mathcal{H}_{w}(\mathcal{G}) \longrightarrow[0, \infty)$ por

$$
\|A\|_{\mathcal{H}_{w}(\mathcal{G})}=\inf \left\{\|C\| \cdot\left\|B_{1}\right\|_{\mathcal{G}} \cdots\left\|B_{m}\right\|_{\mathcal{G}}\right\}
$$

onde o ínfimo é tomado sobre todas as fatorações $C \circ\left(B_{1}, \ldots, B_{m}\right)$ de $A$ por $\mathcal{G}$ em relação a todas partições por meio das quais $A$ se fatora.

Observação 2.3.3. Quando escrevemos

$$
A=C \circ\left(B_{1}, \ldots, B_{m}\right)
$$

com $A$ se fatorando em relação a uma partição $\left\{j_{1}^{(1)}, \ldots, j_{k_{1}}^{(1)}\right\} \cup \cdots \cup\left\{j_{1}^{(m)}, \ldots, j_{k_{m}}^{(m)}\right\}$ de $\{1, \ldots, n\}$, naturalmente estamos efetuando as permutações necessárias para que a igualdade faça sentido. Por exemplo, se tivermos uma aplicação $A: E_{1} \times E_{2} \times E_{3} \times E_{4} \longrightarrow$ $F$ que se fatora por $\mathcal{G}$ em relação à partição $\{1,3\} \cup\{2,4\}$, ao escrevermos

$$
A=C \circ\left(B_{1}, B_{2}\right)
$$

onde $B_{1} \in \mathcal{G}\left(E_{1}, E_{3} ; G_{1}\right), B_{2} \in \mathcal{G}\left(E_{2}, E_{4} ; G_{2}\right)$ e $C \in \mathcal{L}\left(G_{1}, G_{2} ; F\right)$, está subentendido que queremos dizer

$$
A\left(x_{1}, x_{2}, x_{3}, x_{4}\right)=C \circ\left(B_{1}, B_{2}\right)\left(x_{1}, x_{2}, x_{3}, x_{4}\right)=C\left(B_{1}\left(x_{1}, x_{3}\right), B_{2}\left(x_{2}, x_{4}\right)\right) .
$$

O método da fatoração fraco padece de uma dificuldade que salta aos olhos: se uma aplicação $A$ se fatora em relação a uma partição $\Pi_{1}$ e uma aplicação $B$ se fatora em relação a um partição $\Pi_{2} \neq \Pi_{1}$, não há como garantir que $A+B$ se fatora em relação a alguma partição. Ou seja, em geral não há como garantir que as componentes sejam espaços vetoriais. Vejamos que, uma vez superado este problema, as coisas funcionam bem: 
Proposição 2.3.4. Seja $\left(\mathcal{G},\|\cdot\|_{\mathcal{G}}\right)$ hiper-ideal p-normado. Então:

(i) $\mathcal{H}_{w}(\mathcal{G})$ é hiper-ideal se, e somente se, toda componente $\mathcal{H}_{w}(\mathcal{G})\left(E_{1}, \ldots, E_{n} ; F\right)$ é subespaço vetorial de $\mathcal{L}\left(E_{1}, \ldots, E_{n} ; F\right)$.

(ii) Se $\mathcal{H}_{w}(\mathcal{G})$ é hiper-ideal, então $\left(\mathcal{H}_{w}(\mathcal{G}),\|\cdot\|_{\mathcal{H}_{w}(\mathcal{G})}\right)$ é $(p / n)_{n=1}^{\infty}$-normado se, e somente se, $\|\cdot\|_{\mathcal{H}_{w}(\mathcal{G})}$ satisfaz a desigualdade $p / n$-triangular em toda componente $\mathcal{H}_{w}(\mathcal{G})\left(E_{1}, \ldots, E_{n} ; F\right)$.

Demonstração. (i) Uma implicação é óbvia. Para a outra provemos que $\mathcal{H}_{w}(\mathcal{G})$ é um hiper-ideal. Consideremos $M=\varphi_{1} \otimes \cdots \otimes \varphi_{n} \otimes b \in \mathcal{L}\left(E_{1}, \ldots, E_{n} ; F\right)$, onde $\varphi_{i} \in E_{i}^{\prime}$, $i=1, \ldots, n$ e $b \in F$. Sabemos que $\mathcal{G}$ contém as aplicações de tipo finito por ser hiperideal, $\log 0$

$$
M=I_{n} \circ\left(\varphi_{1}, \ldots, \varphi_{n}\right)
$$

é uma fatoração para $M$ por $\mathcal{G}$ em relação à partição $\{1\} \cup \cdots \cup\{n\}$, e portanto $M \in$ $\mathcal{H}_{w}(\mathcal{G})\left(E_{1}, \ldots, E_{n} ; F\right)$.

Mostremos que $\mathcal{H}_{w}(\mathcal{G})$ satisfaz a propriedade de hiper-ideal. Para isso sejam naturais $1 \leq s_{1}<\cdots<s_{n}, A \in \mathcal{H}_{w}(\mathcal{G})\left(E_{1}, \ldots, E_{n} ; F\right), t \in \mathcal{L}(F ; H)$ e $D_{1} \in \mathcal{L}\left(H_{1}, \ldots, H_{s_{1}} ; E_{1}\right)$, $\ldots, D_{n} \in \mathcal{L}\left(H_{s_{n-1}+1}, \ldots, H_{s_{n}} ; E_{n}\right)$. Então existe uma partição de $\{1, \ldots, n\}$, digamos $\left\{j_{1}^{(1)}, \ldots, j_{k_{1}}^{(1)}\right\} \cup \cdots \cup\left\{j_{1}^{(m)}, \ldots, j_{k_{m}}^{(m)}\right\}$, em relação à qual $A$ se fatora por $\mathcal{G}$, digamos

$$
A=C \circ\left(B_{1}, \ldots, B_{m}\right),
$$

onde $B_{i} \in \mathcal{G}\left(E_{j_{1}^{(i)}}, \ldots, E_{j_{k}^{(i)}} ; G_{i}\right), i=1, \ldots, m$ e $C \in \mathcal{L}\left(G_{1}, \ldots, G_{m} ; F\right)$. Disso segue que

$$
\begin{aligned}
t \circ A \circ\left(D_{1}, \ldots, D_{n}\right) & =t \circ\left(C \circ\left(B_{1}, \ldots, B_{m}\right)\right) \circ\left(D_{1}, \ldots, D_{n}\right) \\
& =(t \circ C) \circ\left(B_{1} \circ\left(D_{j_{1}^{(1)}}, \ldots, D_{j_{k_{1}}^{(1)}}\right), \ldots, B_{m} \circ\left(D_{j_{1}^{(m)}}, \ldots, D_{j_{k m}^{(m)}}\right)\right) .
\end{aligned}
$$

Como $\mathcal{G}$ é hiper-ideal, temos

$$
B_{i}\left(D_{j_{1}^{(i)}}, \ldots, D_{j_{k_{i}}^{(i)}}\right) \in \mathcal{G}\left(H_{s_{1}^{(i)}-1}+1, \ldots, H_{s_{k_{i}}} ; G_{i}\right),
$$

para todo $i=1, \ldots, m$, e $t \circ C \in \mathcal{L}\left(G_{1}, \ldots, G_{m} ; H\right)$. Portanto

$$
t \circ A \circ\left(D_{1}, \ldots, D_{n}\right) \in \mathcal{H}_{w}(\mathcal{G})\left(H_{1}, \ldots, H_{m_{n}} ; H\right) .
$$

(ii) Novamente apenas uma implicação demanda demonstração. Suponhamos que a função $\|\cdot\|_{\mathcal{H}_{w}(\mathcal{G})}$ satisfaça a desigualdade $p / n$-triangular em toda componente de $\mathcal{H}_{w}(\mathcal{G})$. Vamos mostrar que $\|\cdot\|_{\mathcal{H}_{w}(\mathcal{G})}$ satisfaz as outras propriedades de norma.

(1) Suponha $A \in \mathcal{H}_{w}(\mathcal{G})$ e $\|A\|_{\mathcal{H}_{w}(\mathcal{G})}=0$. Para toda fatoração $A=C \circ\left(B_{1}, \ldots, B_{m}\right)$ por $\mathcal{G}$ vale que

$$
\|A\| \leq\|C\| \cdot\left\|B_{1}\right\| \cdots\left\|B_{m}\right\| \leq\|C\| \cdot\left\|B_{1}\right\|_{\mathcal{G}} \cdots\left\|B_{m}\right\|_{\mathcal{G}}
$$


então $\|A\| \leq\|A\|_{\mathcal{H}_{w}(\mathcal{G})}$. Disso segue que $A=0$.

(2) Sejam $A \in \mathcal{H}_{w}(\mathcal{G})$ e $\lambda \in \mathbb{K}$. Se $\lambda \neq 0$,

$$
\begin{aligned}
\|\lambda A\|_{\mathcal{H}_{w}(\mathcal{G})} & =\inf \left\{\|\lambda C\| \cdot\left\|B_{1}\right\|_{\mathcal{G}} \cdots\left\|B_{m}\right\|_{\mathcal{G}}\right\} \\
& =|\lambda| \cdot \inf \left\{\|C\| \cdot\left\|B_{1}\right\|_{\mathcal{G}} \cdots\left\|B_{m}\right\|_{\mathcal{G}}\right\}=|\lambda| \cdot\|A\|_{\mathcal{H}_{w}(\mathcal{G})},
\end{aligned}
$$

onde usamos na primeira igualdade que $C \circ\left(B_{1}, \ldots, B_{m}\right)$ é fatoração de $A$ se, e somente se, $(\lambda C) \circ\left(B_{1}, \ldots, B_{m}\right)$ é fatoração de $\lambda A$. Para $\lambda=0$ a igualdade é clara. Com isso a condição $(a)$ da Definição 2.1.2 está satisfeita.

Verifiquemos (b) da Definição 2.1.2. Da desigualdade $\|\cdot\| \leq\|\cdot\|_{\mathcal{H}_{w}(\mathcal{G})}$ provada acima já sabemos que $\left\|I_{n}\right\|_{\mathcal{H}_{w}(\mathcal{G})} \geq 1$. Por outro lado, para cada partição $\left\{j_{1}^{(1)}, \ldots, j_{k_{1}}^{(1)}\right\} \cup \cdots \cup$ $\left\{j_{1}^{(m)}, \ldots, j_{k_{m}}^{(m)}\right\}$ de $\{1, \ldots, n\}$,

$$
I_{m} \circ\left(I_{k_{1}}, \ldots, I_{k_{m}}\right)
$$

é uma fatoração de $I_{n}$ por $\mathcal{G}$ e

$$
\left\|I_{m}\right\| \cdot\left\|I_{k_{1}}\right\|_{\mathcal{G}} \cdots\left\|I_{k_{m}}\right\|_{\mathcal{G}}=1
$$

Disso segue que $\left\|I_{n}\right\|_{\mathcal{H}_{w}(\mathcal{G})} \leq 1$, e portanto vale a igualdade.

(c) Sejam naturais $1 \leq s_{1}<\cdots<s_{n}, A \in \mathcal{H}_{w}(\mathcal{G})\left(E_{1}, \ldots E_{n} ; F\right), t \in \mathcal{L}(F, G)$ e $D_{1} \in \mathcal{L}\left(H_{1}, \ldots H_{s_{1}} ; E_{1}\right), \ldots, D_{n} \in \mathcal{L}\left(H_{s_{n-1}+1}, \ldots, H_{s_{n}} ; E_{n}\right)$. Seja $A=C \circ\left(B_{1}, \ldots, B_{m}\right)$ uma fatoração de $A$ por $\mathcal{G}$ em relação a uma partição $\left\{j_{1}^{(1)}, \ldots, j_{k_{1}}^{(1)}\right\} \cup \cdots \cup\left\{j_{1}^{(m)}, \ldots, j_{k_{m}}^{(m)}\right\}$ de $\{1, \ldots, n\}$. Como vimos na demonstração do item $(i)$ dessa proposição, temos

$$
t \circ A \circ\left(D_{1}, \ldots, D_{n}\right)=(t \circ C)\left(B_{1}\left(D_{j_{1}^{(1)}}, \ldots, D_{j_{k_{1}}^{(1)}}\right), \ldots, B_{m}\left(D_{j_{1}^{(m)}}, \ldots, D_{j_{k_{m}}^{(m)}}\right)\right),
$$

donde segue que

$$
\begin{aligned}
\left\|t \circ A \circ\left(D_{1}, \ldots, D_{n}\right)\right\|_{\mathcal{H}_{w}(\mathcal{G})} \\
\quad \leq\|t \circ C\| \cdot\left\|B_{1}\left(D_{j_{1}^{(1)}}, \ldots, D_{j_{k_{1}}^{(1)}}\right)\right\|_{\mathcal{G}} \cdots\left\|B_{m}\left(D_{j_{1}^{(m)}}, \ldots, D_{j_{k_{m}}^{(m)}}\right)\right\|_{\mathcal{G}} \\
\quad \leq\|t\| \cdot\left(\|C\| \cdot\left\|B_{1}\right\|_{\mathcal{G}} \cdots\left\|B_{m}\right\|_{\mathcal{G}}\right) \cdot\left\|D_{1}\right\| \cdots\left\|D_{n}\right\| .
\end{aligned}
$$

Como a desigualdade vale para qualquer fatoração de $A$ por $\mathcal{G}$, segue que

$$
\left\|t \circ A \circ\left(D_{1}, \ldots, D_{n}\right)\right\|_{\mathcal{H}_{w}(\mathcal{G})} \leq\|t\| \cdot\|A\|_{\mathcal{H}_{w}(\mathcal{G})}\left\|D_{1}\right\| \cdots\left\|D_{n}\right\| .
$$


Está claro que o método da fatoração fraco apresenta resultados apenas parciais, pois: (i) não podemos garantir que as componentes de $\mathcal{H}_{w}(\mathcal{G})$ sejam espaços vetoriais mesmo que $\mathcal{G}$ seja hiper-ideal; (ii) mesmo supondo que as componentes são espaços vetoriais, não sabemos se a quasi-norma $\|\cdot\|_{\mathcal{H}_{w}(\mathcal{G})}$ é completa.

Analisemos agora a classe $\mathcal{H}_{s}(\mathcal{G})$ gerada pelo método da fatoração forte. Como o próximo resultado deixa claro, este método parece ser mais promissor:

Lema 2.3.5. Se $\mathcal{G}$ é um hiper-ideal, então as componentes de $\mathcal{H}_{s}(\mathcal{G})$ são subespaços vetoriais que contêm as aplicações multilineares de posto finito.

Demonstração. Sejam $A_{1}, A_{2} \in \mathcal{H}_{s}(\mathcal{G})\left(E_{1}, \ldots, E_{n} ; F\right)$. Fixada uma partição de $\{1, \ldots, n\}$, a saber $\left\{j_{1}^{(1)}, \ldots, j_{k_{1}}^{(1)}\right\} \cup \cdots \cup\left\{j_{1}^{(m)}, \ldots, j_{k_{m}}^{(m)}\right\}$, existem espaços de Banach $G_{11}, \ldots, G_{1 m}$, $G_{21}, \ldots, G_{2 m}$ e aplicações $B_{1 l} \in \mathcal{G}\left(E_{j_{1}^{(l)}}, \ldots, E_{j_{k_{i}}^{(l)}} ; G_{1 l}\right), B_{2 l} \in \mathcal{G}\left(E_{j_{1}^{(l)}}, \ldots, E_{j_{k_{i}}} ; G_{2 l}\right), l=$ $i, \ldots, m, C_{1} \in \mathcal{L}\left(G_{11}, \ldots, G_{1 m} ; F\right)$ e $C_{2} \in \mathcal{L}\left(G_{21}, \ldots, G_{2 m} ; F\right)$ tais que

$$
A_{1}=C_{1} \circ\left(B_{11}, \ldots, B_{1 m}\right) \text { e } A_{2}=C_{2} \circ\left(B_{21}, \ldots, B_{2 m}\right) .
$$

Para cada $l=1, \ldots, m$, defina $G_{l}:=G_{1 l} \times G_{2 l}$ munido de qualquer uma das normas usuais do produto cartesiano. Consideremos então as inclusões $\iota_{1 l}: G_{1 l} \longrightarrow G_{l}, \iota_{2 l}: G_{2 l} \longrightarrow G_{l}$, e as projeções $\pi_{1 l}: G_{l} \longrightarrow G_{1 l}, \pi_{2 l}: G_{l} \longrightarrow G_{2 l}$, para $l=1, \ldots, m$. Como $\mathcal{G}$ é hiper-ideal temos

$$
B_{l}:=\iota_{1 l} B_{1 l}+\iota_{2 l} B_{2 l} \in \mathcal{G}\left(E_{j_{1}^{(l)}}, \ldots, E_{j_{k_{l}}(l)} ; G_{l}\right) .
$$

Por outro lado, definimos $C: G_{1} \times \cdots \times G_{m} \longrightarrow F$ por

$$
C=C_{1} \circ\left(\pi_{11}, \ldots, \pi_{1 m}\right)+C_{2} \circ\left(\pi_{21}, \ldots, \pi_{2 m}\right) .
$$

Então $C \in \mathcal{L}\left(G_{1}, \ldots, G_{m} ; F\right)$ e

$$
\begin{aligned}
& C \circ\left(B_{1}, \ldots, B_{m}\right) \\
&=\left(C_{1} \circ\left(\pi_{11}, \ldots, \pi_{1 m}\right)+C_{2} \circ\left(\pi_{21}, \ldots, \pi_{2 m}\right)\right) \circ\left(\iota_{11} B_{11}+\iota_{21} B_{21}, \ldots, \iota_{1 m} B_{1 m}+\iota_{2 m} B_{2 m}\right) \\
&= C_{1} \circ\left(\pi_{11} \circ\left(\iota_{11} B_{11}+\iota_{21} B_{21}\right), \ldots, \pi_{1 m} \circ\left(\iota_{1 m} B_{1 m}+\iota_{2 m} B_{2 m}\right)\right) \\
&+C_{2} \circ\left(\pi_{21} \circ\left(\iota_{11} B_{11}+\iota_{21} B_{21}\right), \ldots, \pi_{2 m} \circ\left(\iota_{1 m} B_{1 m}+\iota_{2 m} B_{2 m}\right)\right) \\
&= C_{1} \circ\left(B_{11}, \ldots, B_{1 m}\right)+C_{2} \circ\left(B_{21}, \ldots, B_{2 m}\right)=A_{1}+A_{2} .
\end{aligned}
$$

Como a partição é arbitrária segue que $A+A^{\prime} \in \mathcal{H}_{s}(\mathcal{G})\left(E_{1}, \ldots, E_{n} ; F\right)$.

Dado $\lambda \in \mathbb{K}$, podemos escrever

$$
\lambda A=\lambda\left(C \circ\left(B_{1}, \ldots, B_{m}\right)\right)=(\lambda C) \circ\left(B_{1}, \ldots, B_{m}\right),
$$


o que prova que $\lambda A \in \mathcal{H}_{s}(\mathcal{G})\left(E_{1}, \ldots, E_{n} ; F\right)$.

Consideremos $D=\varphi_{1} \otimes \cdots \otimes \varphi_{n} \otimes b \in \mathcal{L}\left(E_{1}, \ldots, E_{n} ; F\right)$ uma aplicação $n$-linear de tipo finito elementar. Para cada partição $\left\{j_{1}^{(1)}, \ldots, j_{k_{1}}^{(1)}\right\} \cup \cdots \cup\left\{j_{1}^{(m)}, \ldots, j_{k_{m}}^{(m)}\right\}$ de $\{1, \ldots, n\}$, podemos fatorar $D$ na forma

$$
D=B_{m} \circ\left(T_{1}, \ldots, T_{m}\right),
$$

onde, para cada $l=1, \ldots, m, T_{l}: E_{j_{1}^{(l)}} \times \cdots \times E_{j_{k}^{(l)}} \longrightarrow \mathbb{K}$ é dada por

$$
T_{l}\left(x_{j_{1}^{(l)}}, \ldots, x_{j_{k}^{(l)}}\right)=\varphi_{j_{1}^{(l)}}\left(x_{j_{1}^{(l)}}\right) \cdots \varphi_{j_{k}^{(l)}}\left(x_{j_{k}^{(l)}}\right)
$$

e

$$
B_{m}: \mathbb{K}^{m} \longrightarrow F, B_{m}\left(\lambda_{1}, \ldots, \lambda_{m}\right)=\lambda_{1} \cdots \lambda_{m} b .
$$

É claro que cada $T_{l} \in \mathcal{G}\left(E_{j_{1}^{(l)}}, \ldots, E_{j_{k_{l}}^{(l)}}\right)$ por ser aplicação multilinear de tipo finito, e portanto $D \in \mathcal{H}_{s}(\mathcal{G})\left(E_{1}, \ldots, E_{n} ; F\right)$.

Dados operadores lineares $u_{i}: E_{i} \longrightarrow F_{i}, i=1, \ldots, n$, definindo

$$
u_{1} \times \cdots \times u_{n}: E_{1} \times \cdots \times E_{n} \longrightarrow F_{1} \widehat{\otimes}_{\pi} \cdots \widehat{\otimes}_{\pi} F_{n}
$$

por

$$
u_{1} \times \cdots \times u_{n}\left(x_{1}, \ldots, x_{n}\right)=u_{1}\left(x_{1}\right) \otimes \cdots \otimes u_{n}\left(x_{n}\right),
$$

obtemos um operador $n$-linear contínuo e $\left\|u_{1} \times \cdots \times u_{n}\right\|=\left\|u_{1}\right\| \cdots\left\|u_{n}\right\|$.

Consideremos as seguinte condição sobre uma classe $\mathcal{G}$ de aplicações multilineares:

(c) Se $u_{i} \in \mathcal{G}\left(E_{i} ; F_{i}\right), i=1, \ldots, n$, então $u_{1} \times \cdots \times u_{n} \in \mathcal{G}\left(E_{1}, \ldots, E_{n} ; F_{1} \widehat{\otimes}_{\pi} \cdots \widehat{\otimes}_{\pi} F_{n}\right)$.

O resultado abaixo diz que, na presença da condição $(\mathfrak{c})$, o método da fatoração forte para hiper-ideais se reduz ao método da fatoração para multi-ideais.

Lema 2.3.6. Se a classe de aplicações multilineares $\mathcal{G}$ satisfaz a condição (c), então $\mathcal{H}_{s}(\mathcal{G})=\mathcal{L}\left(\mathcal{G}^{1}\right)$, onde $\mathcal{G}^{1}$ é a componente linear de $\mathcal{G}$; em particular $\mathcal{H}_{s}(\mathcal{G})$ é um multiideal.

Demonstração. Considerando a partição trivial $\{1, \ldots, n\}=\{1\} \cup \cdots \cup\{n\}$, é imediato que $\mathcal{H}_{s}(\mathcal{G}) \subseteq \mathcal{L}\left(\mathcal{G}^{1}\right)$

Reciprocamente, dada $A \in \mathcal{L}\left(\mathcal{G}^{1}\right)\left(E_{1}, \ldots, E_{n} ; F\right)$ existem $u_{1} \in \mathcal{G}\left(E_{1} ; F_{1}\right), \ldots, u_{n} \in$ $\mathcal{G}\left(E_{n} ; F_{n}\right)$ e $B \in \mathcal{L}\left(G_{1}, \ldots, G_{n} ; F\right)$ tais que

$$
A=B \circ\left(u_{1}, \ldots, u_{n}\right) .
$$


Seja $\left\{j_{1}^{(1)}, \ldots, j_{k_{1}}^{(1)}\right\} \cup \cdots \cup\left\{j_{1}^{(m)}, \ldots, j_{k_{m}}^{(m)}\right\}$ uma partição de $\{1, \ldots, n\}$. Consideremos, para cada $l=1, \ldots, m, U_{l}: E_{j_{1}^{(l)}} \times \cdots \times E_{j_{k_{l}}^{(l)}} \longrightarrow G_{j_{1}^{(l)}} \widehat{\otimes}_{\pi} \cdots \widehat{\otimes}_{\pi} G_{j_{k_{l}}^{(l)}}$, dada por

$$
U_{l}\left(x_{j_{1}^{(l)}}, \ldots, x_{j_{k_{l}}^{(l)}}\right)=u_{j_{1}^{(l)}}\left(x_{j_{1}^{(l)}}\right) \otimes \cdots \otimes u_{j_{k_{l}}^{(l)}}\left(x_{j_{k_{l}}(l)}\right)
$$

isto é, $U_{l}=u_{j_{1}^{(l)}} \times \cdots \times u_{j_{k_{l}}^{(l)}}$. Como $\mathcal{G}$ satisfaz $(\mathfrak{c})$, decorre que

$$
U_{l} \in \mathcal{G}\left(E_{j_{1}^{(l)}}, \ldots, E_{j_{k_{l}}^{(l)}} ; G_{j_{1}^{(l)}} \widehat{\otimes}_{\pi} \cdots \widehat{\otimes}_{\pi} G_{j_{k_{l}}^{(l)}}\right), l=1, \ldots, m .
$$

Consideremos a linearização $B_{L} \in \mathcal{L}\left(G_{1} \widehat{\otimes}_{\pi} \cdots \widehat{\otimes}_{\pi} G_{n} ; F\right)$ de $B$. Da associatividade da norma projetiva, a aplicação natural

$$
I:\left(G_{j_{1}^{(1)}} \widehat{\otimes}_{\pi} \cdots \widehat{\otimes}_{\pi} G_{j_{k_{1}}^{(1)}}\right) \widehat{\otimes}_{\pi} \cdots \widehat{\otimes}_{\pi}\left(G_{j_{1}^{(m)}} \widehat{\otimes}_{\pi} \cdots \widehat{\otimes}_{\pi} G_{j_{k_{m}}}\right) \longrightarrow G_{1} \widehat{\otimes}_{\pi} \cdots \widehat{\otimes}_{\pi} G_{n}
$$

é um isomorfismo isométrico. Então

$$
B_{L} \circ I \in \mathcal{L}\left(\left(G_{j_{1}^{(1)}} \widehat{\otimes}_{\pi} \cdots \widehat{\otimes}_{\pi} G_{j_{k_{1}}^{(1)}}\right) \widehat{\otimes}_{\pi} \cdots \widehat{\otimes}_{\pi}\left(G_{j_{1}^{(m)}} \widehat{\otimes}_{\pi} \cdots \widehat{\otimes}_{\pi} G_{j_{k_{m}}^{(m)}}\right) ; F\right) .
$$

Pela propriedade universal do produto tensorial projetivo (Teorema 1.4.3), existe $C \in$ $\mathcal{L}\left(G_{j_{1}^{(1)}} \widehat{\otimes}_{\pi} \cdots \widehat{\otimes}_{\pi} G_{j_{k_{1}}^{(1)}}, \ldots, G_{j_{1}^{(m)}} \widehat{\otimes}_{\pi} \cdots \widehat{\otimes}_{\pi} G_{j_{k_{m}}^{(m)}} ; F\right)$ tal que $C_{L}=B_{L} \circ I$. Então,

$$
\begin{aligned}
C\left(y_{j_{1}^{(1)}} \otimes \cdots \otimes y_{j_{1}}^{(1)}\right. & \left.\ldots, y_{j_{1}^{(m)}} \otimes \cdots \otimes y_{j_{k_{m}}^{(m)}}\right) \\
& =C_{L}\left(\left(y_{j_{1}^{(1)}} \otimes \cdots \otimes y_{j_{k_{1}}^{(1)}}\right) \otimes \cdots \otimes\left(y_{j_{1}^{(m)}} \otimes \cdots \otimes y_{j_{k_{m}}^{(m)}}\right)\right) \\
& =B_{L} \circ I\left(\left(y_{j_{1}^{(1)}} \otimes \cdots \otimes y_{j_{k_{1}}^{(1)}}\right) \otimes \cdots \otimes\left(y_{j_{1}^{(m)}} \otimes \cdots \otimes y_{j_{k_{m}}^{(m)}}\right)\right) \\
& =B_{L}\left(y_{1} \otimes \cdots \otimes y_{n}\right)=B\left(y_{1}, \ldots, y_{n}\right)
\end{aligned}
$$

para todos $y_{1}, \ldots, y_{n}$. Assim,

$$
\begin{aligned}
C \circ\left(U_{1}, \ldots, U_{m}\right)\left(x_{1}, \ldots, x_{n}\right) \\
=C\left(U_{1}\left(x_{j_{1}^{(1)}}, \ldots, x_{j_{k_{1}}}\right), \ldots, U_{m}\left(x_{j_{1}^{(m)}}, \ldots, x_{j_{k_{m}}^{(m)}}\right)\right) \\
=C\left(u_{j_{1}^{(1)}}\left(x_{j_{1}^{(1)}}\right) \otimes \cdots \otimes u_{j_{k_{1}}^{(1)}}\left(x_{j_{k_{1}}^{(1)}}\right), \ldots, u_{j_{1}^{(m)}}\left(x_{j_{1}^{(m)}}\right) \otimes \cdots \otimes u_{j_{k_{m}}^{(m)}}\left(x_{j_{k_{m}}(m)}\right)\right) \\
=B\left(u_{1}\left(x_{1}\right), \ldots, u_{n}\left(x_{n}\right)\right)=A\left(x_{1}, \ldots, x_{n}\right)
\end{aligned}
$$

para todos $x_{1}, \ldots, x_{n}$, onde a penúltima igualdade segue de (2.6). Portanto $A \in \mathcal{H}_{s}(\mathcal{G})$. 
Com isso concluímos que, mesmo se $\mathcal{G}$ for hiper-ideal, em geral $\mathcal{H}_{s}(\mathcal{G})$ é apenas um multi-ideal, que pode não ser um hiper-ideal. De fato, consideremos $\mathcal{G}=\mathcal{L}_{\mathcal{F}}$, o hiper-ideal das aplicações de posto finito. Sabemos que $\mathcal{L}(\mathcal{F})=\mathcal{L}_{f}$, onde $\mathcal{F}$ é o ideal dos operadores de tipo finito (veja, por exemplo, [16, Proposition 2.3.5]). Das propriedades descritas na Proposição 1.4 .1 segue imediatamente que $\mathcal{L}_{\mathcal{F}}$ satisfaz a condição $(\mathfrak{c})$, então

$$
\mathcal{H}_{s}\left(\mathcal{L}_{\mathcal{F}}\right)=\mathcal{L}\left(\mathcal{L}_{\mathcal{F}}^{1}\right)=\mathcal{L}(\mathcal{F})=\mathcal{L}_{f}
$$

onde usamos o Lema 2.3.6 na segunda igualdade. Mas, como vimos no Exemplo 2.2.1, $\mathcal{L}_{f}$ não é hiper-ideal.

Fornecemos a seguir uma contribuição desta tese para a teoria de multi-ideais:

Teorema 2.3.7. (a) Se $\mathcal{G}$ é um multi-ideal, então $\mathcal{H}_{s}(\mathcal{G})$ é um multi-ideal.

(b) Suponha que a seguinte condição esteja satisfeita: para toda aplicação n-linear $A$ pertencente a $\mathcal{H}_{s}(\mathcal{G})$ e para toda partição $\Pi$ de $\{1, \ldots, n\}$, tem-se

$$
\|A\|_{\mathcal{H}_{w}(\mathcal{G})}=\inf \left\{\|C\| \cdot\left\|B_{1}\right\|_{\mathcal{G}} \cdots\left\|B_{m}\right\|_{\mathcal{G}}\right\}
$$

onde o ínfimo é tomado sobre todas as fatorações $C \circ\left(B_{1}, \ldots, B_{m}\right)$ de $A$ por $\mathcal{G}$ em relação a П. Neste caso, se $\left(\mathcal{G},\|\cdot\|_{\mathcal{G}}\right)$ é um multi-ideal p-Banach, então $\left(\mathcal{H}_{s}(\mathcal{G}),\|\cdot\|_{\mathcal{H}_{w}(\mathcal{G})}\right)$ é um multi-ideal $(p / n)_{n=1}^{\infty}$-Banach.

Demonstração. (a) Do Lema 2.3.5 sabemos que as componentes de $\mathcal{H}_{s}(\mathcal{G})$ são subespaços vetoriais que contêm as aplicações multilineares de tipo finito.

Verifiquemos a propriedade de multi-ideal. Sejam $A \in \mathcal{H}_{s}(\mathcal{G})\left(E_{1}, \ldots, E_{n} ; F\right), u_{i} \in$ $\mathcal{L}\left(H_{l} ; E_{l}\right), l=1, \ldots, n$ e $t \in \mathcal{L}(F ; H)$. Seja $\left\{j_{1}^{(1)}, \ldots, j_{k_{1}}^{(1)}\right\} \cup \cdots \cup\left\{j_{1}^{(m)}, \ldots, j_{k_{m}}^{(m)}\right\}$ uma partição de $\{1, \ldots, n\}$. Como $A \in \mathcal{H}_{s}(\mathcal{G})\left(E_{1}, \ldots, E_{n} ; F\right)$, existem espaços de Banach $G_{1}, \ldots, G_{m}$ e aplicações multilineares

$$
B_{i} \in \mathcal{G}\left(E_{j_{1}^{(i)}}, \ldots, E_{j_{k_{i}}^{(i)}} ; G_{i}\right), i=1, \ldots, m, \text { e } C \in \mathcal{L}\left(G_{1}, \ldots, G_{m} ; F\right)
$$

tais que $A=C \circ\left(B_{1}, \ldots, B_{m}\right)$. Então,

$$
t \circ A \circ\left(u_{1}, \ldots, u_{n}\right)=(t \circ C) \circ\left(B_{1} \circ\left(u_{1}, \ldots, u_{j_{k_{1}}^{(1)}}\right), \ldots, B_{m} \circ\left(u_{j_{1}^{(m)}}, \ldots, u_{j_{k_{m}}^{(m)}}\right)\right) .
$$

Como $\mathcal{G}$ é multi-ideal, temos

$$
B_{l} \circ\left(u_{j_{1}^{(l)}}, \ldots, u_{j_{k_{l}}(l)}\right) \in \mathcal{G}\left(H_{j_{1}^{(l)}}, \ldots, H_{j_{k_{l}}} ; G_{l}\right), l=1, \ldots, m .
$$


Então (2.7) é uma fatoração para $t \circ A \circ\left(u_{1}, \ldots, u_{n}\right)$ em relação à partição fixada. Como se trata de uma partição arbitrária, concluímos que

$$
t \circ A \circ\left(u_{1}, \ldots, u_{n}\right) \in \mathcal{H}_{s}(\mathcal{G})\left(H_{1}, \ldots, H_{n} ; H\right),
$$

o que nos permite concluir que $\mathcal{H}_{s}(\mathcal{G})$ é um multi-ideal.

(b) Mostremos agora que, dentro das hipóteses especificadas, $\left(\mathcal{H}_{s}(\mathcal{G}),\|\cdot\|_{\mathcal{H}_{w}(\mathcal{G})}\right)$ é um multi-ideal $(p / n)_{n=1}^{\infty}$-Banach. Observamos que, exceto pela desigualdade $(p / n)_{n=1}^{\infty}$ triangular, todas as outras propriedades de $(p / n)_{n=1}^{\infty}$-norma foram verificadas na demonstração do item (ii) da Proposição 2.3.4, e nada é afetado por estarmos com um multi-ideal ao invés de um hiper-ideal. Pelo item (a) já sabemos que $I_{n} \in \mathcal{H}_{s}(\mathcal{G})$. A demonstração de que $\left\|I_{n}\right\|_{\mathcal{H}_{w}(\mathcal{G})}=1$ é idêntica à que foi feita na demonstração da Proposição 2.3.4, e também neste caso nada muda por termos um multi-ideal no lugar de um hiper-ideal. Então basta provar a condição (c) da Definição 1.5.2 e o item (iv) do Teorema 1.5.3.

Começamos pelo item $(i v)$ do Teorema 1.5.3. Para isso seja $\left(A_{j}\right)_{j=1}^{\infty}$ uma sequência em $\mathcal{H}_{s}(\mathcal{G})\left(E_{1}, \ldots, E_{n} ; F\right)$ tal que $\sum_{j=1}^{\infty}\left\|A_{j}\right\|_{\mathcal{H}_{w}(\mathcal{G})}^{p / n}<\infty$. Devemos verificar que $A:=\sum_{j=1}^{\infty} A_{j} \in$ $\mathcal{H}_{s}(\mathcal{G})\left(E_{1}, \ldots, E_{n} ; F\right)$ e $\|A\|_{\mathcal{H}_{w}(\mathcal{G})}^{p / n} \leq \sum_{j \in 1}^{\infty}\left\|A_{j}\right\|_{\mathcal{H}_{w}(\mathcal{G})}^{p / n}$. Vejamos primeiramente que tal aplicação $A$ está bem definida. Como $\frac{p}{n} \leq p \leq 1$, temos

$$
\sum_{j=1}^{\infty}\left\|A_{j}\right\| \leq\left(\sum_{j=1}^{\infty}\left\|A_{j}\right\|^{p / n}\right)^{n / p} \leq\left(\sum_{j=1}^{\infty}\left\|A_{j}\right\|_{\mathcal{H}_{w}(\mathcal{G})}^{p / n}\right)^{n / p}<\infty .
$$

Portanto $A:=\sum_{j=1}^{\infty} A_{j} \in \mathcal{L}\left(E_{1}, \ldots, E_{n} ; F\right)$.

Fixemos agora $\Pi=\left\{r_{1}^{(1)}, \ldots, r_{k_{1}}^{(1)}\right\} \cup \cdots \cup\left\{r_{1}^{(m)}, \ldots, r_{k_{m}}^{(m)}\right\}$ uma partição de $\{1, \ldots, n\}$. Dado $\varepsilon>0$, por hipótese, para cada $j$,

$$
\left\|A_{j}\right\|_{\mathcal{H}_{w}(\mathcal{G})}=\inf \left\{\left\|C^{j}\right\| \cdot\left\|B_{1}^{j}\right\|_{\mathcal{G}} \cdots\left\|B_{m}^{j}\right\|_{\mathcal{G}}\right\}
$$

onde o ínfimo é tomado sobre todas as fatorações $C^{j} \circ\left(B_{1}^{j}, \ldots, B_{m}^{j}\right)$ de $A_{j}$ em relação a П. Então existem espaços de Banach $G_{1}, \ldots, G_{m}$, aplicações $B_{i}^{j} \in \mathcal{G}\left(E_{r_{1}^{(i)}}, \ldots, E_{r_{k_{i}}^{(i)}} ; G_{i}^{j}\right)$, $i=1, \ldots, m$, e $C^{j} \in \mathcal{L}\left(G_{1}^{j}, \ldots, G_{m}^{j} ; F\right)$ tais que $A_{j}=C^{j} \circ\left(B_{1}^{j}, \ldots, B_{m}^{j}\right)$ e

$$
\left\|C^{j}\right\| \cdot\left\|B_{1}^{j}\right\|_{\mathcal{G}} \cdots\left\|B_{m}^{j}\right\|_{\mathcal{G}}<(1+\varepsilon)\left\|A_{j}\right\|_{\mathcal{H}_{w}(\mathcal{G})}=: t_{j}
$$

Podemos supor, sem perda de generalidade, que $\left\|C^{j}\right\| \leq 1$ e $\left\|B_{i}^{j}\right\|_{\mathcal{G}} \leq t_{j}^{1 / m}, i=1, \ldots, m$. De fato, sendo $A_{j} \neq 0$, temos $C^{j}, B_{1}^{j}, \ldots, B_{m}^{j} \neq 0$. Então basta escrevermos

$$
A_{j}=\left(\frac{\left\|B_{1}^{j}\right\|_{\mathcal{G}} \cdots\left\|B_{m}^{j}\right\|_{\mathcal{G}}}{t_{j}} \cdot C^{j}\right) \circ\left(\frac{t_{j}^{1 / m}}{\left\|B_{1}^{j}\right\|_{\mathcal{G}}} \cdot B_{1}^{j}, \ldots, \frac{t_{j}^{1 / m}}{\left\|B_{m}^{j}\right\|_{\mathcal{G}}} \cdot B_{m}^{j}\right) .
$$


Definimos $G_{i}:=\left(\bigoplus_{j \in \mathbb{N}} G_{i}^{j}\right)_{1}, i=1, \ldots, m$. Sejam $\iota_{i j}: G_{i}^{j} \longrightarrow G_{i}$ e $\pi_{i j}: G_{i} \longrightarrow G_{i}^{j}$ as inclusões e projeções canônicas, respectivamente. Para $z_{i}=\left(z_{i}^{j}\right)_{j=1}^{\infty} \in G_{i}$, temos:

$$
\begin{aligned}
\sum_{j=1}^{\infty}\left\|C^{j} \circ\left(\pi_{1 j}, \ldots, \pi_{m j}\right)\left(z_{1}, \ldots, z_{m}\right)\right\| & =\sum_{j=1}^{\infty}\left\|C^{j}\left(z_{1}^{j}, \ldots, z_{m}^{j}\right)\right\| \\
& \leq \sum_{j=1}^{\infty}\left\|C^{j}\right\| \cdot\left\|z_{1}^{j}\right\| \cdots\left\|z_{m}^{j}\right\| \\
& \leq\left(\sum_{j=1}^{\infty}\left\|z_{1}^{j}\right\|\right) \ldots\left(\sum_{j=1}^{\infty}\left\|z_{m}^{j}\right\|\right) \\
& =\left\|z_{1}\right\|_{1} \cdots\left\|z_{m}\right\|_{1} .
\end{aligned}
$$

Então $C:=\sum_{j=1}^{\infty} C^{j} \circ\left(\pi_{1 j}, \ldots, \pi_{m j}\right) \in \mathcal{L}\left(G_{1}, \ldots, G_{m} ; F\right)$ e $\|C\| \leq 1$. Por outro lado, para cada $i=1, \ldots, m,\left(\iota_{i j} \circ B_{i}^{j}\right)_{j=1}^{\infty}$ é uma sequência em $\mathcal{G}$ tal que

$$
\sum_{j=1}^{\infty}\left\|\iota_{i j} \circ B_{i}^{j}\right\|_{\mathcal{G}}^{p} \leq \sum_{j=1}^{\infty}\left\|B_{i}^{j}\right\|_{\mathcal{G}}^{p} \leq \sum_{j=1}^{\infty} t_{j}^{p / m}=(1+\varepsilon)^{p / m}\left(\sum_{j=1}^{\infty}\left\|A_{j}\right\|_{\mathcal{H}_{w}(\mathcal{G})}^{p / m}\right)<\infty
$$

Como $\left(\mathcal{G},\|\cdot\|_{\mathcal{G}}\right)$ é um multi-ideal $p$-Banach, pelo Critério da Série para multi-ideais (Teorema 1.5.3) segue que

$$
B_{i}:=\sum_{j=1}^{\infty} \iota_{i j} \circ B_{i}^{j} \in \mathcal{G}\left(E_{r_{1}^{(i)}}, \ldots, E_{r_{k_{i}}^{(i)}}, G_{i}\right) \text { e }\left\|B_{i}\right\|_{\mathcal{G}}^{p} \leq \sum_{j=1}^{\infty}\left\|\iota_{i j} \circ B_{i}^{j}\right\|_{\mathcal{G}}^{p} \leq \sum_{j=1}^{\infty} t_{j}^{p / m} .
$$

Como $\|\cdot\| \leq\|\cdot\|_{\mathcal{G}}$, a convergência $B_{i}=\sum_{j=1}^{\infty} \iota_{i j} \circ B_{i}^{j}$ também se dá na norma uniforme. Então obtemos

$$
\begin{aligned}
C \circ\left(B_{1}, \ldots, B_{m}\right) & =\sum_{j=1}^{\infty} C^{j} \circ\left(\pi_{1 j} \circ B_{1}, \ldots, \pi_{m j} \circ B_{m}\right) \\
& =\sum_{j=1}^{\infty} C^{j} \circ\left(\pi_{1 j} \circ\left(\sum_{k=1}^{\infty} \iota_{1 k} \circ B_{1}^{k}\right), \ldots, \pi_{m j} \circ\left(\sum_{k=1}^{\infty} \iota_{m k} \circ B_{m}^{k}\right)\right) \\
& =\sum_{j=1}^{\infty} C^{j} \circ\left(\sum_{k=1}^{\infty} \pi_{1 j} \circ \iota_{1 k} \circ B_{1}^{k}, \ldots, \sum_{k=1}^{\infty} \pi_{m j} \circ \iota_{m k} \circ B_{m}^{k}\right) \\
& =\sum_{j=1}^{\infty} C^{j} \circ\left(B_{1}^{j}, \ldots, B_{m}^{j}\right)=\sum_{j=1}^{\infty} A_{j}=A .
\end{aligned}
$$


Como a partição $\Pi$ de $\{1, \ldots, n\}$ foi escolhida sem qualquer restrição, concluímos que $A \in \mathcal{H}_{s}(\mathcal{G})\left(E_{1}, \ldots, E_{n} ; F\right)$. Por hipótese,

$$
\|A\|_{\mathcal{H}_{w}(\mathcal{G})}=\inf \left\{\left\|C^{\prime}\right\| \cdot\left\|B_{1}^{\prime}\right\|_{\mathcal{G}} \cdots\left\|B_{m}^{\prime}\right\|_{\mathcal{G}}\right\}
$$

onde o ínfimo é tomado sobre todas as fatorações $C^{\prime} \circ\left(B_{1}^{\prime}, \ldots, B_{m}^{\prime}\right)$ de $A$ em relação a $\Pi$. Disso e de $\frac{p}{n} \leq \frac{p}{m}$, segue que

$$
\begin{aligned}
\|A\|_{\mathcal{H}_{w}(\mathcal{G})}^{p / n} & =\inf \left\{\left\|C^{\prime}\right\| \cdot\left\|B_{1}^{\prime}\right\|_{\mathcal{G}} \cdots\left\|B_{m}^{\prime}\right\|_{\mathcal{G}}\right\}^{p / n} \leq\|C\|^{p / n} \cdot\left\|B_{1}\right\|_{\mathcal{G}}^{p / n} \cdots\left\|B_{m}\right\|_{\mathcal{G}}^{p / n} \\
& \leq\left(\sum_{j=1}^{\infty} t_{j}^{p / m}\right)^{1 / n} \cdots\left(\sum_{j=1}^{\infty} t_{j}^{p / m}\right)^{1 / n}=\left(\sum_{j=1}^{\infty} t_{j}^{p / m}\right)^{m / n}=\left(\left\|\left(t_{j}\right)_{j=1}^{\infty}\right\|_{p / m}\right)^{\frac{p}{m} \cdot \frac{m}{n}} \\
& =\left(\left\|\left(t_{j}\right)_{j=1}^{\infty}\right\|_{p / m}\right)^{p / n} \leq\left(\left\|\left(t_{j}\right)_{j=1}^{\infty}\right\|_{p / n}\right)^{p / n}=\sum_{j=1}^{\infty} t_{j}^{p / n} \\
& =(1+\varepsilon)^{p / n}\left(\sum_{j=1}^{\infty}\left\|A_{j}\right\|_{\mathcal{H}_{w}(\mathcal{G})}^{p / n}\right)
\end{aligned}
$$

onde o ínfimo após a primeira igualdade é tomado sobre todas as fatorações de $A$ em relação a $\Pi$. Fazendo $\varepsilon \longrightarrow 0$, o resultado segue.

Resta apenas provar a condição (c) da Definição 1.5.2. De acordo com a Proposição 2.3.4, resta apenas a desigualdade de multi-ideal. Por meio de (2.7), da definição de $\|\cdot\|_{\mathcal{H}_{w}(\mathcal{G})}$ e por propriedades da $p$-norma $\|\cdot\|_{\mathcal{G}}$, tem-se

$$
\begin{aligned}
\| t \circ A \circ\left(u_{1}, \ldots,\right. & \left.u_{n}\right) \|_{\mathcal{H}_{w}(\mathcal{G})} \\
& \leq\|t \circ C\| \cdot\left\|B_{1} \circ\left(u_{1}, \ldots, u_{j_{k_{1}}^{(1)}}\right)\right\|_{\mathcal{G}} \cdots\left\|B_{m} \circ\left(u_{j_{1}^{(m)}}, \ldots, u_{j_{k_{m}}^{(m)}}\right)\right\|_{\mathcal{G}} \\
& \leq\|t\| \cdot\left(\|C\| \cdot\left\|B_{1}\right\|_{\mathcal{G}} \cdots\left\|B_{m}\right\|_{\mathcal{G}}\right) \cdot\left\|u_{1}\right\| \cdots\left\|u_{n}\right\| .
\end{aligned}
$$

Pela arbitrariedade da fatoração e da partição, segue que

$$
\left\|t \circ A \circ\left(u_{1}, \ldots, u_{n}\right)\right\|_{\mathcal{H}_{w}(\mathcal{G})} \leq\|t\| \cdot\|A\|_{\mathcal{H}_{w}(\mathcal{G})} \cdot\left\|u_{1}\right\| \cdots\left\|u_{n}\right\| .
$$

Cientes de que os métodos da fatoração fraco e fatoração forte apresentam resultados aquém dos desejados, devemos buscar refinamentos desses métodos que levem, eventualmente, a resultados mais satisfatórios. Uma estratégia é tentar obter hiper-ideais impondo uma condição sobre o comprimento da partição em relação à qual as aplicações se fatoram, visando garantir que as componentes da classe sejam espaços vetoriais, e ao mesmo 
tempo tornar possível a adaptação da demonstração da completude via Critério da Série (Teorema 2.1.9). Duas possibilidades se apresentam nesta direção:

(i) Impor a condição de que o mínimo entre os comprimentos das partições em relação às quais duas aplicações multilineares distintas de $\mathcal{H}_{w}(\mathcal{G})$ se fatoram seja o comprimento de uma partição em relação à qual as duas se fatoram. Dessa forma estaria garantida a condição de que as componentes de $\mathcal{H}_{w}(\mathcal{G})$ sejam espaços vetoriais. Entretanto, não é difícil verificar que a classe gerada dessa forma coincide, novamente, com o multi-ideal $\mathcal{L}\left(\mathcal{G}^{1}\right)$, que, como vimos, não é um hiper-ideal em geral.

(ii) Tomar o caminho inverso e exigir que o máximo entre os comprimentos das partições em relação às quais duas aplicações multilineares distintas de $\mathcal{H}_{w}(\mathcal{G})$ se fatoram seja o comprimento de uma partição em relação à qual as duas se fatoram. Assim também resolveríamos o problema da preservação da soma. Mas, nesse caso, a classe gerada coincidiria com $\mathcal{G}$ se esta for um hiper-ideal; e com isso não estaríamos criando um novo hiper-ideal a partir de um hiper-ideal dado.

Diante dos problemas apresentados pelos métodos da fatoração fraco e fatoração forte, e da insuficiência das estratégias esboçadas acima, concluímos que, para a geração de hiper-ideais, o método da fatoração precisa ter sua filosofia alterada. Acreditamos que tudo o que fizemos até agora nesta seção justifica esta opção. Apresentamos a seguir uma classe, gerada por uma adaptação da filosofia do método da fatoração, que apresenta resultados bem mais satisfatórios para a geração de hiper-ideais:

Definição 2.3.8. Seja $\mathcal{G}$ uma subclasse da classe das aplicações multilineares contínuas entre espaços de Banach. Dados $n \in \mathbb{N}$ e espaços de Banach $E_{1}, \ldots, E_{n}, F$, definimos $\mathcal{L}(\mathcal{G})\left(E_{1}, \ldots, E_{n} ; F\right)$ como o espaço gerado pelas aplicações multilineares que podem ser escritas na forma

$$
A=C \circ\left(B_{1}, \ldots, B_{m}\right),
$$

onde $\left\{j_{1}^{(1)}, \ldots, j_{k_{1}}^{(1)}\right\} \cup \cdots \cup\left\{j_{1}^{(m)}, \ldots, j_{k_{m}}^{(m)}\right\}$ é uma partição de $\{1, \ldots, n\}$, para $l=1, \ldots, m$, $B_{l} \in \mathcal{G}\left(E_{j_{1}^{(l)}}, \ldots, E_{j_{k_{l}}(l)} ; G_{l}\right)$, e $C \in \mathcal{L}\left(G_{1}, \ldots, G_{m} ; F\right)$.

Se a cada componente $\mathcal{G}\left(E_{1}, \ldots, E_{n} ; F\right)$ de $\mathcal{G}$ está associada uma função $\|\cdot\|_{\mathcal{G}}: \mathcal{G}\left(E_{1}, \ldots, E_{n} ; F\right) \longrightarrow[0, \infty)$, definimos ainda $\|\cdot\|_{\mathcal{L}(\mathcal{G})}: \mathcal{L}(\mathcal{G}) \longrightarrow[0, \infty)$ por

$$
\|A\|_{\mathcal{L}(\mathcal{G})}=\inf \left\{\sum_{i=1}^{s}\left\|C_{i}\right\| \cdot\left\|B_{j_{1}^{(i)}}\right\|_{\mathcal{G}} \cdots\left\|B_{j_{k_{i}}}\right\|_{\mathcal{G}}\right\},
$$

onde o ínfimo é tomado sobre todas as representações de $A$ na forma $A=\sum_{i=1}^{s} C_{i}$ 。 $\left(B_{j_{1}^{(i)}}, \ldots, B_{j_{k_{i}}^{(i)}}\right)$, onde $B_{j_{1}^{(i)}}, \ldots, B_{j_{k_{i}}^{(i)}}$ pertencem a $\mathcal{G}$ para todo $i$. 
Teorema 2.3.9. Se $\left(\mathcal{G},\|\cdot\|_{\mathcal{G}}\right)$ é um hiper-ideal p-normado, então $\left(\mathcal{L}(\mathcal{G}),\|\cdot\|_{\mathcal{L}(\mathcal{G})}\right)$ é um hiper-ideal normado que contém $\mathcal{G}$.

Demonstração. (1) Pela definição de $\mathcal{L}(\mathcal{G})$, cada componente $\mathcal{L}(\mathcal{G})\left(E_{1}, \ldots, E_{n} ; F\right)$ é um subespaço vetorial de $\mathcal{L}\left(E_{1}, \ldots, E_{n} ; F\right)$ que contém $\mathcal{G}\left(E_{1}, \ldots, E_{n} ; F\right)$. Como $\mathcal{G}$ contém as aplicações multilineares de tipo finito, por ser um hiper-ideal, então $\mathcal{L}(\mathcal{G})$ também as contém.

(2) Se $1 \leq m_{1}<\cdots<m_{n}, A \in \mathcal{L}(\mathcal{G})\left(E_{1}, \ldots, E_{n} ; F\right), D_{1} \in \mathcal{L}\left(G_{1}, \ldots, G_{m_{1}} ; E_{1}\right), \ldots, D_{n} \in$ $\mathcal{L}\left(G_{m_{n-1}+1}, \ldots, G_{m_{n}} ; E_{n}\right)$ e $t \in \mathcal{L}(F ; H)$, então podemos escrever $A=\sum_{i=1}^{s} C_{i} \circ\left(B_{j_{1}^{(i)}}, \ldots, B_{j_{k_{i}}^{(i)}}\right)$, onde $B_{j_{1}^{(i)}}, \ldots, B_{j_{k_{i}}^{(i)}}$ são elementos de $\mathcal{G}$ para todo $i=1, \ldots, s$. Daí,

$$
\begin{aligned}
t \circ A \circ\left(D_{1}, \ldots, D_{n}\right) & =t \circ\left(\sum_{i=1}^{s} C_{i} \circ\left(B_{j_{1}^{(i)}}, \ldots, B_{j_{k_{i}}^{(i)}}\right) \circ\left(D_{1}, \ldots, D_{n}\right)\right) \\
& =\sum_{i=1}^{s} t \circ C_{i} \circ\left(B_{j_{1}^{(i)}} \circ\left(D_{1}, \ldots, D_{j_{1}^{(i)}}\right), \ldots, B_{j_{k_{i}}^{(i)}} \circ\left(D_{j_{k_{i-1}}^{(i-1)}+1}, \ldots, D_{n}\right)\right) .
\end{aligned}
$$

Como $\mathcal{G}$ é hiper-ideal,

$$
B_{j_{1}^{(i)}} \circ\left(D_{1}, \ldots, D_{j_{1}^{(i)}}\right), \ldots, B_{j_{k_{i}}^{(i)}} \circ\left(D_{j_{k_{i-1}}^{(i-1)}+1}, \ldots, D_{n}\right)
$$

são elementos de $\mathcal{G}$ para todo $i=1, \ldots, s$. Portanto

$$
t \circ A \circ\left(D_{1}, \ldots, D_{n}\right) \in \mathcal{L}(\mathcal{G})\left(G_{1}, \ldots, G_{m_{n}} ; H\right)
$$

Mostremos que $\|\cdot\|_{\mathcal{L}(\mathcal{G})}$ é uma norma. Dada $A=\sum_{i=1}^{s} C_{i} \circ\left(B_{j_{1}^{(i)}}, \ldots, B_{j_{k_{i}}^{(i)}}\right.$, temos

$$
\|A\| \leq \sum_{i=1}^{s}\left\|C_{i}\right\| \cdot\left\|B_{j_{1}^{(i)}}\right\| \cdots\left\|B_{j_{k_{i}}^{(i)}}\right\| \leq \sum_{i=1}^{s}\left\|C_{i}\right\| \cdot\left\|B_{j_{1}^{(i)}}\right\|_{\mathcal{G}} \cdots\left\|B_{j_{k_{i}}^{(i)}}\right\|_{\mathcal{G}}
$$

Como esta majoração vale para toda representação de $A$, segue que $\|A\| \leq\|A\|_{\mathcal{L}(\mathcal{G})}$. Dessa forma, se $\|A\|_{\mathcal{L}(\mathcal{G})}=0$, então $A=0$. A recíproca é imediata.

Notemos que, para $\lambda \neq 0$, temos que $\sum_{i=1}^{s} C_{i} \circ\left(B_{j_{1}^{(i)}}, \ldots, B_{j_{k_{i}}^{(i)}}\right)$ é representação de $A$ se, 
e somente se, $\sum_{i=1}^{s} \lambda C_{i} \circ\left(B_{j_{1}^{(i)}}, \ldots, B_{j_{k_{i}}^{(i)}}\right)$ é representação de $\lambda A$. Daí,

$$
\begin{aligned}
\|\lambda A\|_{\mathcal{L}(\mathcal{G})} & =\inf \left\{\sum_{i=1}^{s}\left\|\lambda C_{i}\right\| \cdot\left\|B_{j_{1}^{(i)}}\right\|_{\mathcal{G}} \cdots\left\|B_{j_{k_{i}}^{(i)}}\right\|_{\mathcal{G}}\right\} \\
& =\inf \left\{\sum_{i=1}^{s}|\lambda| \cdot\left\|C_{i}\right\| \cdot\left\|B_{j_{1}^{(i)}}\right\|_{\mathcal{G}} \cdots\left\|B_{j_{k_{i}}^{(i)}}\right\|_{\mathcal{G}}\right\} \\
& =|\lambda| \cdot \inf \left\{\sum_{i=1}^{s}\left\|C_{i}\right\| \cdot\left\|B_{j_{1}^{(i)}}\right\|_{\mathcal{G}} \cdots\left\|B_{j_{k_{i}}^{(i)}}\right\|_{\mathcal{G}}\right\} \\
& =|\lambda| \cdot\|A\|_{\mathcal{L}(\mathcal{G})},
\end{aligned}
$$

onde o ínfimo é tomado sobre todas as representações de $A$. Para $\lambda=0$ a igualdade é imediata.

Sejam $A, A^{\prime} \in \mathcal{L}(\mathcal{G})\left(E_{1}, \ldots, E_{n} ; F\right)$. Então, para quaisquer representações $\sum_{i=1}^{s_{1}} C_{i} \circ$ $\left(B_{j_{1}^{(i)}}, \ldots, B_{j_{k_{i}}^{(i)}}\right)$ de $A$ e $\sum_{i=1}^{s_{2}} C_{i}^{\prime} \circ\left(B_{j_{1}^{(i)}}^{\prime}, \ldots, B_{j_{k_{i}}^{(i)}}^{\prime}\right)$ de $A^{\prime}$, a soma

$$
\sum_{i=1}^{s_{1}} C_{i} \circ\left(B_{j_{1}^{(i)}}, \ldots, B_{j_{k_{i}}^{(i)}}\right)+\sum_{i=1}^{s_{2}} C_{i}^{\prime} \circ\left(B_{j_{1}^{(i)}}^{\prime}, \ldots, B_{j_{k_{i}}^{(i)}}^{\prime}\right)
$$

é uma representação para $A+A^{\prime}$. Então

$$
\left\|A+A^{\prime}\right\|_{\mathcal{L}(\mathcal{G})} \leq \sum_{i=1}^{s_{1}}\left\|C_{i}\right\| \cdot\left\|B_{j_{1}^{(i)}}\right\|_{\mathcal{G}} \cdots\left\|B_{j_{k_{i}}^{(i)}}\right\|_{\mathcal{G}}+\sum_{i=1}^{s_{2}}\left\|C_{i}^{\prime}\right\| \cdot\left\|B_{j_{1}^{(i)}}^{\prime}\right\|_{\mathcal{G}} \cdots\left\|B_{j_{k_{i}}^{(i)}}^{\prime}\right\|_{\mathcal{G}}
$$

Como as representações de $A$ e $A^{\prime}$ são arbitrárias, tem-se

$$
\left\|A+A^{\prime}\right\|_{\mathcal{L}(\mathcal{G})} \leq\|A\|_{\mathcal{L}(\mathcal{G})}+\left\|A^{\prime}\right\|_{\mathcal{L}(\mathcal{G})}
$$

provando que $\|\cdot\|_{\mathcal{L}(\mathcal{G})}$ é uma norma.

Verifiquemos que $\left\|I_{n}\right\|_{\mathcal{L}(\mathcal{G})}=1$. Já sabemos que

$$
1=\left\|I_{n}\right\| \leq\left\|I_{n}\right\|_{\mathcal{L}(\mathcal{G})}
$$

Por outro lado, $I_{n}$ pode ser vista como representação de si mesma, uma vez que pertence ao hiper-ideal $\mathcal{G}$, então

$$
\left\|I_{n}\right\|_{\mathcal{L}(\mathcal{G})} \leq\left\|I_{n}\right\|_{\mathcal{G}}=1
$$

donde segue a igualdade.

Resta mostrar a desigualdade de hiper-ideal. Seguindo as conclusões de (2), sabemos 
que dada uma representação $\sum_{i=1}^{s} C_{i} \circ\left(B_{j_{1}^{(i)}}, \ldots, B_{j_{k_{i}}^{(i)}}\right)$ de $A$ em $\mathcal{L}(\mathcal{G})\left(E_{1}, \ldots, E_{n} ; F\right)$,

$$
\sum_{i=1}^{s}\left(t \circ C_{i}\right) \circ\left(B_{j_{1}^{(i)}}\left(D_{1}, \ldots, D_{j_{1}^{(i)}}\right), \ldots, B_{j_{k_{i}}^{(i)}}\left(D_{j_{k_{i-1}}^{(i-1)}+1}, \ldots, D_{n}\right)\right)
$$

é uma representação de $t \circ A \circ\left(D_{1}, \ldots, D_{n}\right)$. Disso segue que

$$
\begin{aligned}
\left\|t \circ A \circ\left(D_{1}, \ldots, D_{n}\right)\right\|_{\mathcal{L}(\mathcal{G})} & \\
& \left.\leq \sum_{i=1}^{s}\left\|t \circ C_{i}\right\| \cdot\left\|B_{j_{1}^{(i)}}\left(D_{1}, \ldots, D_{j_{1}^{(i)}}\right)\right\|_{\mathcal{G}} \cdots \| B_{j_{k_{i}}}\left(D_{j_{k_{i-1}}^{(i-1)}+1}, \ldots, D_{n}\right)\right) \|_{\mathcal{G}} \\
& \leq\|t\| \cdot\left(\sum_{i=1}^{s}\left\|C_{i}\right\| \cdot\left\|B_{j_{1}^{(i)}}\right\|_{\mathcal{G}} \cdots\left\|B_{j_{k_{i}}^{(i)}}\right\|_{\mathcal{G}}\right) \cdot\left\|D_{1}\right\| \cdots\left\|D_{n}\right\|,
\end{aligned}
$$

onde usamos na última desigualdade que $\|\cdot\|_{\mathcal{G}}$ satisfaz a desigualdade de hiper-ideal. Como a representação de $A$ é arbitrária,

$$
\left\|t \circ A \circ\left(D_{1}, \ldots, D_{n}\right)\right\|_{\mathcal{L}(\mathcal{G})} \leq\|t\| \cdot\|A\|_{\mathcal{L}(\mathcal{G})} \cdot\left\|D_{1}\right\| \cdots\left\|D_{n}\right\|
$$

Dessa forma, apesar de não termos a completude, esta última classe $\mathcal{L}(\mathcal{G})$ apresenta resultados bem melhores, pois nenhuma condição é imposta para que suas componentes sejam espaços vetoriais.

No caso de multi-ideais, o método da fatoração gera classes que, em geral, são menores que classes geradas por outros métodos (veja, por exemplo, [16, Proposition 2.3.4] ou [7, Proposition 11(b)]). Abaixo damos um critério para decidir quando a classe $\mathcal{L}(\mathcal{G})$ está contida em um determinado multi-ideal, e portanto quando está contida em um determinado hiper-ideal.

Proposição 2.3.10. Sejam $\mathcal{G}$ e $\mathcal{H}$ classes de aplicações multilineares contínuas entre espaços de Banach. Considere as seguintes condições:

(a) $\mathcal{L}(\mathcal{G}) \subseteq \mathcal{H}$.

(b) Se $A_{i} \in \mathcal{G}\left(E_{n_{i-1}+1}, \ldots, E_{n_{i}} ; F_{i}\right)$, com $0=n_{0}<n_{1} \cdots<n_{k}$ e $i=1, \ldots, k$, então definido $A_{1} \times \cdots \times A_{k}: E_{1} \times \cdots \times E_{n_{k}} \longrightarrow F_{1} \widehat{\otimes}_{\pi} \cdots \widehat{\otimes}_{\pi} F_{k}$ por

$$
\left(A_{1} \times \cdots \times A_{k}\right)\left(x_{1}, \ldots, x_{n_{k}}\right)=A_{1}\left(x_{1}, \ldots, x_{n_{1}}\right) \otimes \cdots \otimes A_{k}\left(x_{n_{k-1}+1}, \ldots, x_{n_{k}}\right),
$$

tem-se $A_{1} \times \cdots \times A_{k} \in \mathcal{H}\left(E_{1}, \ldots, E_{n_{k}} ; F_{1} \widehat{\otimes}_{\pi} \cdots \widehat{\otimes}_{\pi} F_{k}\right)$.

Então $(a) \Longrightarrow(b)$ e, se $\mathcal{H}$ é multi-ideal, então $(a)$ e (b) são equivalentes. 
Demonstração. $(a) \Longrightarrow(b)$ Nas condições de $(b)$, como $A_{i}$ pertente à classe $\mathcal{G}$ para todo $i=1, \ldots, k$, segue

$$
A_{1} \times \cdots \times A_{k}=\sigma_{k} \circ\left(A_{1}, \ldots, A_{k}\right) \in \mathcal{L}(\mathcal{G}) \subseteq \mathcal{H}
$$

onde $\sigma_{k}$ é a aplicação multilinear canônica (veja Teorema 1.4.3).

Suponhamos que $\mathcal{H}$ seja um multi-ideal e provemos que, neste caso, $(b) \Longrightarrow(a)$ : Seja $A$ uma aplicação multilinear pertencente à classe $\mathcal{L}(\mathcal{G})$ com representação $A=\sum_{i=1}^{s} C_{i}$ ○ $\left(B_{j_{1}^{(i)}}, \ldots, B_{j_{k_{i}}^{(i)}}\right)$. Podemos escrever

$$
C_{i} \circ\left(B_{j_{1}^{(i)}}, \ldots, B_{j_{k_{i}}^{(i)}}\right)=\left(C_{i}\right)_{L} \circ\left(B_{j_{1}^{(i)}} \times \cdots \times B_{j_{k_{i}}^{(i)}}\right),
$$

para $i=1, \ldots, s$. Como $B_{j_{1}^{(i)}}, \ldots, B_{j_{k_{i}}^{(i)}}$ são elementos de $\mathcal{G}$, por $(b)$ segue que $B_{j_{1}^{(i)}} \times \cdots \times$ $B_{j_{k_{i}}^{(i)}}$ pertence à classe $\mathcal{H}$. Sendo $\mathcal{H}$ um multi-ideal, concluímos que

$$
A=\sum_{i=1}^{s}\left(C_{i}\right)_{L} \circ\left(B_{j_{1}^{(i)}} \times \cdots \times B_{j_{k_{i}}^{(i)}}\right)
$$

pertence à classe $\mathcal{H}$, como queríamos.

\subsection{Ideais de composição}

Veremos nesta seção que, ao contrário do método da fatoração, os multi-ideais de composição (Definição 1.5.5) são perfeitamente adaptados ao conceito de hiper-ideais. Isso nos permitirá concluir que mais algumas classes clássicas de aplicações multilineares são exemplos de hiper-ideais. Assim como na definição de hiper-ideal fechado (Definição 2.1.4), um ideal de operadores $\mathcal{I}$ cujas componentes são fechadas em relação à norma uniforme é chamado de ideal fechado, ou seja, $(\mathcal{I},\|\cdot\|)$ é um ideal de Banach.

Teorema 2.4.1. Se $\mathcal{I}$ é um ideal de operadores, então $\mathcal{I} \circ \mathcal{L}$ é um hiper-ideal. Se $\left(\mathcal{I},\|\cdot\|_{\mathcal{I}}\right)$ é ideal de operadores $p$-normado (p-Banach, respectivamente), então $\left(\mathcal{I} \circ \mathcal{L},\|\cdot\|_{\mathcal{I} \circ \mathcal{L})}\right.$ é hiper-ideal p-normado (p-Banach, respectivamente). Em particular, se $\mathcal{I}$ é fechado, então $\mathcal{I} \circ \mathcal{L}$ é um hiper-ideal fechado.

Demonstração. (1) Como já sabemos que $\mathcal{I} \circ \mathcal{L}$ é um multi-ideal, então suas componentes são espaços vetoriais que contêm as aplicações multilineares de tipo finito.

(2) Consideremos $B_{1} \in \mathcal{L}\left(G_{1}, \ldots, G_{m_{1}} ; E_{1}\right), \ldots, B_{n} \in \mathcal{L}\left(G_{m_{n-1}+1}, \ldots, G_{m_{n}} ; E_{n}\right)$, onde $m_{1}<\cdots<m_{n}, A \in \mathcal{I} \circ \mathcal{L}\left(E_{1}, \ldots, E_{n} ; F\right)$ e $t \in \mathcal{L}(F ; H)$. Podemos escrever $A=v \circ C$, 
onde $C \in \mathcal{L}\left(E_{1}, \ldots, E_{n} ; F_{1}\right)$ e $v \in \mathcal{I}\left(F_{1} ; F\right)$. Definindo $u:=t \circ v \in \mathcal{I}\left(F_{1} ; H\right)$ e $D:=$ $C \circ\left(B_{1}, \ldots, B_{n}\right) \in \mathcal{L}\left(G_{1}, \ldots, G_{m_{n}} ; F_{1}\right)$, segue que

$$
t \circ A \circ\left(B_{1}, \ldots, B_{n}\right)=t \circ(v \circ C) \circ\left(B_{1}, \ldots, B_{n}\right)=(t \circ v) \circ\left(C \circ\left(B_{1}, \ldots, B_{n}\right)\right)=u \circ D,
$$

o que prova que $t \circ A \circ\left(B_{1}, \ldots, B_{n}\right) \in \mathcal{I} \circ \mathcal{L}\left(G_{1}, \ldots, G_{m_{n}} ; H\right)$.

Como já sabemos que $\left(\mathcal{I} \circ \mathcal{L},\|\cdot\|_{\mathcal{I} \circ \mathcal{L}}\right)$ é um multi-ideal $p$-normado ( $p$-Banach, respectivamente) no caso em que $\left(\mathcal{I},\|\cdot\|_{\mathcal{I}}\right)$ é ideal de operadores $p$-normado ( $p$-Banach, respectivamente), resta verificar apenas a condição $(c)$ da Definição 2.1.2. Com efeito, segundo a notação do parágrafo anterior, temos

$$
\begin{aligned}
\left\|t \circ A \circ\left(B_{1}, \ldots, B_{n}\right)\right\|_{\mathcal{I} \circ \mathcal{L}} & \leq\|t \circ v\|_{\mathcal{I}} \cdot\left\|C \circ\left(B_{1}, \ldots, B_{n}\right)\right\| \\
& \leq\|t\| \cdot\|v\|_{\mathcal{I}} \cdot\|C\| \cdot\left\|B_{1}\right\| \cdots\left\|B_{n}\right\| .
\end{aligned}
$$

Como a representação $A=v \circ C$ é arbitrária, segue que

$$
\left\|t \circ A \circ\left(B_{1}, \ldots, B_{n}\right)\right\|_{\mathcal{I} \circ \mathcal{L}} \leq\|t\| \cdot\|A\|_{\mathcal{I} \circ \mathcal{L}} \cdot\left\|B_{1}\right\| \cdots\left\|B_{n}\right\| .
$$

Exemplo 2.4.2. Dizemos que uma aplicação multilinear $A \in \mathcal{L}\left(E_{1}, \ldots, E_{n} ; F\right)$ é compacta (fracamente compacta, respectivamente), se $A\left(B_{E_{1}}, \ldots, B_{E_{n}}\right)$ é um subconjunto relativamente compacto (relativamente fracamente compacto, respectivamente) de $F$. Chamamos de $\mathcal{K}$ e $\mathcal{W}$ os ideais dos operadores compactos e fracamente compactos, respectivamente, e de $\mathcal{L}_{\mathcal{K}}$ e $\mathcal{L}_{\mathcal{W}}$ as classes das aplicações multilineares compactas e fracamente compactas, respectivamente. Pełczyński [40] provou que

$$
\mathcal{L}_{\mathcal{K}}=\mathcal{K} \circ \mathcal{L} \text { e } \mathcal{L}_{\mathcal{W}}=\mathcal{W} \circ \mathcal{L}
$$

Sabendo que essas classes são fechadas na norma uniforme, segue do Teorema 2.4.1 que as classes das aplicações multilineares compactas e fracamente compactas são hiper-ideais fechados.

Estudaremos em seguida então outra classe bem estudada (veja, por exemplo, [2, 14, 20]).

Exemplo 2.4.3. Dizemos que uma aplicação multilinear $A \in \mathcal{L}\left(E_{1}, \ldots, E_{n} ; F\right)$ é fracamente sequencialmente contínua se para quaisquer sequências $\left(x_{j}^{l}\right)_{j=1}^{\infty} \subseteq E_{l}, l=1, \ldots, n$, que convergem fracamente, digamos para $x_{l} \in E_{l}$, respectivamente, tivermos

$$
A\left(x_{j}^{1}, \ldots, x_{j}^{n}\right) \longrightarrow A\left(x_{1}, \ldots, x_{n}\right),
$$


onde a convergência é na norma de $F$. A classe das aplicações multilineares fracamente sequencialmente contínua é denotada por $\mathcal{L}_{w s c}$. No caso linear, a literatura consagrou o termo operador linear completamente contínuo, e usaremos o símbolo $\mathcal{C C}$ para denotar o ideal formado por esses operadores. Ou seja, $\mathcal{C C}=\mathcal{L}_{w s c}^{1}$. Usando o fato que operadores lineares são contínuos em relação às topologias fracas no domínio e no contra-domínio [12, Proposição 6.2.9], é imediato que $\mathcal{L}_{w s c}$ é um multi-ideal. Por outro lado, vejamos que $\mathcal{L}_{w s c}$ não é um hiper-ideal.

De fato, consideremos a aplicação

$$
A: \ell_{2} \times \ell_{2} \longrightarrow \ell_{1}, A\left(\left(x_{j}\right)_{j=1}^{\infty},\left(y_{j}\right)_{j=1}^{\infty}\right)=\left(x_{j} y_{j}\right)_{j=1}^{\infty}
$$

Claramente $A$ é uma aplicação bilinear e da desigualdade de Hölder segue que $A$ é contínua. Mais ainda, $\|A\| \leq 1$. Notemos que $A \notin \mathcal{L}_{w s c}\left({ }^{2} \ell_{2} ; \ell_{1}\right)$. Para isso seja $\left(e_{i}\right)_{i=1}^{\infty}$ a sequência dos vetores unitários canônicos em $\ell_{2}$. É sabido que $\left(e_{i}\right)_{i=1}^{\infty}$ converge fracamente para zero em $\ell_{2}$. Agora, pela definição de $A, A\left(e_{i}, e_{i}\right)=e_{i}$ para todo $i$, e se $A \in \mathcal{L}_{w s c}\left({ }^{2} \ell_{2} ; \ell_{1}\right)$, então $\left(A\left(e_{i}, e_{i}\right)\right)_{i=1}^{\infty}$ deveria convergir para $A(0,0)=0$ em norma. Mas isso não ocorre, visto que $\left\|e_{i}\right\|_{1}=1$ para todo $i \in \mathbb{N}$. Portanto $A \notin \mathcal{L}_{w s c}\left({ }^{2} \ell_{2} ; \ell_{1}\right)$.

Por outro lado, sabemos pelo Teorema de Schur [12, Teorema 6.2.12] que $I d_{\ell_{1}} \in$ $\mathcal{C C}\left(\ell_{1} ; \ell_{1}\right)$. Então, se $\mathcal{L}_{w s c}$ fosse hiper-ideal teríamos ter $A=\operatorname{Id}_{\ell_{1}} \circ A \in \mathcal{L}_{w s c}\left({ }^{2} \ell_{2} ; \ell_{1}\right)$, o que não ocorre.

Introduzimos a seguir uma classe que adapta o multi-ideal $\mathcal{L}_{w s c}$ para a teoria de hiperideais.

Definição 2.4.4. Sejam $E_{1}, \ldots, E_{n}$ espaços de Banach. Dizemos que uma sequência $\left(\left(x_{j}^{1}, \ldots, x_{j}^{n}\right)\right)_{j=1}^{\infty}$ em $E_{1} \times \cdots \times E_{n}$ converge multilinearmente para $\left(x_{1}, \ldots, x_{n}\right) \in E_{1} \times$ $\cdots \times E_{n}$ se a sequência $\left(T\left(x_{j}^{1}, \ldots, x_{j}^{n}\right)\right)_{j=1}^{\infty}$ converge para $T\left(x_{1}, \ldots, x_{n}\right)$ para toda forma $n$-linear $T \in \mathcal{L}\left(E_{1}, \ldots, E_{n}\right)$. Dizemos ainda que a sequência $\left(\left(x_{j}^{1}, \ldots, x_{j}^{n}\right)\right)_{j=1}^{\infty}$ é multilinearmente convergente se existe $\left(x_{1}, \ldots, x_{n}\right) \in E_{1} \times \cdots \times E_{n}$ de modo que $\left(\left(x_{j}^{1}, \ldots, x_{j}^{n}\right)\right)_{j=1}^{\infty}$ converge multilinearmente para $\left(x_{1}, \ldots, x_{n}\right)$.

Chamamos uma aplicação $A \in \mathcal{L}\left(E_{1}, \ldots, E_{n} ; F\right)$ de multilinearmente sequencialmente contínua se, para toda sequência $\left(\left(x_{j}^{1}, \ldots, x_{j}^{n}\right)\right)_{j=1}^{\infty}$ em $E_{1} \times \cdots \times E_{n}$ que converge multilinearmente, digamos para $\left(x_{1}, \ldots, x_{n}\right)$, tivermos

$$
A\left(x_{j}^{1}, \ldots, x_{j}^{n}\right) \longrightarrow A\left(x_{1}, \ldots, x_{n}\right)
$$

na norma de $F$. Neste caso escrevemos $A \in \mathcal{L}_{m s c}\left(E_{1}, \ldots, E_{n} ; F\right)$. 
É claro que esta classe também generaliza, para o caso multilinear, os operadores completamente contínuos, isto é, $\mathcal{C C}=\mathcal{L}_{m s c}^{1}$.

A convergência multilinear não apresenta todas as características de uma convergência usual; vejamos, por exemplo, que a convergência multilinear não implica que as sequências de cada uma das coordenadas são limitadas e que não há unicidade do limite multilinear:

Exemplo 2.4.5. Dado um espaço de Banach $E$, tome $0 \neq x \in E$. Para toda forma bilinear $T \in \mathcal{L}\left({ }^{2} E\right)$,

$$
T\left(j x, \frac{x}{j^{2}}\right)=\frac{1}{j} T(x, x) \stackrel{j}{\longrightarrow} 0=T(0, y)=T(y, 0)
$$

para todo $y \in E$. Assim, $\left(j x, \frac{x}{j^{2}}\right)_{j=1}^{\infty}$ converge bilinearmente para $(0, y)$ e para $(y, 0)$, qualquer que seja $y \in E$. Mais ainda, a sequência $(j x)_{j=1}^{\infty}$ não é limitada em $E$.

Para o nossos propósitos, a seguinte limitação será suficiente:

Lema 2.4.6. Seja $\left(\left(x_{j}^{1}, \ldots, x_{j}^{n}\right)\right)_{j=1}^{\infty} \subseteq E_{1} \times \cdots \times E_{n}$ uma sequência multilinearmente convergente para $\left(x_{1}, \ldots, x_{n}\right)$. Então existe $K>0$ tal que $\left\|x_{j}^{1}\right\| \cdots\left\|x_{j}^{n}\right\| \leq K$ para todo $j \in \mathbb{N}$, e portanto $\left\|x_{1}\right\| \cdots\left\|x_{n}\right\| \leq K$.

Demonstração. Seja $\varphi \in\left(E_{1} \widehat{\otimes} \cdots \widehat{\otimes} E_{n}\right)^{\prime}$. Pela Proposição 1.4 .6 sabemos que existe $B \in$ $\mathcal{L}\left(E_{1}, \ldots, E_{n}\right)$ tal que $B_{L}=\varphi$. Da convergência multilinear de $\left(\left(x_{j}^{1}, \ldots, x_{j}^{n}\right)\right)_{j=1}^{\infty}$ temos

$$
\begin{aligned}
\varphi\left(x_{j}^{1} \otimes \cdots \otimes x_{j}^{n}\right) & =B_{L}\left(x_{j}^{1} \otimes \cdots \otimes x_{j}^{n}\right) \\
& =B\left(x_{j}^{1}, \ldots, x_{j}^{n}\right) \longrightarrow B\left(x_{1}, \ldots, x_{n}\right) \\
& =B_{L}\left(x_{1} \otimes \cdots \otimes x_{n}\right)=\varphi\left(x_{1} \otimes \cdots \otimes x_{n}\right) .
\end{aligned}
$$

Como $\varphi$ é um funcional linear qualquer em $E_{1} \widehat{\otimes} \cdots \widehat{\otimes} E_{n}$, concluímos que a sequência $\left(x_{j}^{1} \otimes \cdots \otimes x_{j}^{n}\right)_{j=1}^{\infty}$ converge fracamente para $x_{1} \otimes \cdots \otimes x_{n}$. Sabemos que toda sequência fracamente convergente é limitada (ver [12, Proposição 6.2.5]), então $\left(x_{j}^{1} \otimes \cdots \otimes x_{j}^{n}\right)_{j=1}^{\infty}$ é limitada em $E_{1} \widehat{\otimes} \cdots \widehat{\otimes} E_{n}$, ou seja, existe $K>0$ tal que

$$
\left\|x_{j}^{1}\right\| \cdots\left\|x_{j}^{n}\right\|=\pi\left(x_{j}^{1} \otimes \cdots \otimes x_{j}^{n}\right) \leq K
$$

para todo $j \in \mathbb{N}$. Também por [12, Proposição 6.2.5] e pela limitação acima segue que

$$
\left\|x_{1}\right\| \cdots\left\|x_{n}\right\|=\pi\left(x_{1} \otimes \cdots \otimes x_{n}\right) \leq \liminf _{j}\left\|x_{j}^{1}\right\| \cdots\left\|x_{j}^{n}\right\| \leq K .
$$


Teorema 2.4.7. A classe $\mathcal{L}_{m s c}$ das aplicações multilinearmente sequencialmente contínuas é um hiper-ideal fechado.

Demonstração. (1) Sejam $A, B \in \mathcal{L}_{m s c}\left(E_{1}, \ldots, E_{n} ; F\right)$ e $\left(\left(x_{j}^{1}, \ldots, x_{j}^{n}\right)\right)_{j=1}^{\infty} \subseteq E_{1} \times \cdots \times E_{n}$ convergindo multilinearmente para $\left(x_{1}, \ldots, x_{n}\right)$. Então $\left(A\left(x_{j}^{1}, \ldots, x_{j}^{n}\right)\right)_{j=1}^{\infty}$ converge em norma para $A\left(x_{1}, \ldots, x_{n}\right)$ e $\left(B\left(x_{j}^{1}, \ldots, x_{j}^{n}\right)\right)_{j=1}^{\infty}$ converge em norma para $B\left(x_{1}, \ldots, x_{n}\right)$. Segue que

$$
\left((A+\lambda B)\left(x_{j}^{1}, \ldots, x_{j}^{n}\right)\right)_{j=1}^{\infty}=\left(A\left(x_{j}^{1}, \ldots, x_{j}^{n}\right)+\lambda B\left(x_{j}^{1}, \ldots, x_{j}^{n}\right)\right)_{j=1}^{\infty}
$$

converge em norma para

$$
A\left(x_{1}, \ldots, x_{n}\right)+\lambda B\left(x_{1}, \ldots, x_{n}\right)=(A+\lambda B)\left(x_{1}, \ldots, x_{n}\right) .
$$

Portanto $A+\lambda B \in \mathcal{L}_{m s c}\left(E_{1}, \ldots, E_{n} ; F\right)$.

Agora, sejam $T \in \mathcal{L}\left(E_{1}, \ldots, E_{n}\right)$ e $y \in F$. Se $\left(\left(x_{j}^{1}, \ldots, x_{j}^{n}\right)\right)_{j=1}^{\infty} \subseteq E_{1} \times \cdots \times E_{n}$ converge multilinearmente para $\left(x_{1}, \ldots, x_{n}\right)$, em particular é verdade que

$$
T\left(x_{j}^{1}, \ldots, x_{j}^{n}\right) \longrightarrow T\left(x_{1}, \ldots, x_{n}\right)
$$

em $\mathbb{K}$. Disso segue trivialmente que

$$
(T \otimes y)\left(x_{j}^{1}, \ldots, x_{j}^{n}\right)=T\left(x_{j}^{1}, \ldots, x_{j}^{n}\right) y \longrightarrow T\left(x_{1}, \ldots, x_{n}\right) y=(T \otimes y)\left(x_{1}, \ldots, x_{n}\right)
$$

na norma de $F$. Então $T \otimes y \in \mathcal{L}_{m s c}\left(E_{1}, \ldots, E_{n} ; F\right)$. Disso concluímos que as aplicações de posto finito, que são da forma $\sum_{i} T_{i} \otimes y_{i}$, são multilinearmente sequencialmente contínuas. $\operatorname{Logo} \mathcal{L}_{m s c}\left(E_{1}, \ldots, E_{n} ; F\right)$ é um espaço vetorial que contém as aplicações de posto finito.

(2) Consideremos $B_{1} \in \mathcal{L}\left(G_{1}, \ldots, G_{m_{1}} ; E_{1}\right), \ldots, B_{n} \in \mathcal{L}\left(G_{m_{n-1}+1}, \ldots, G_{m_{n}} ; E_{n}\right)$, onde $1 \leq m_{1}<\cdots<m_{n}, A \in \mathcal{L}_{m s c}\left(E_{1}, \ldots, E_{m} ; F\right)$ e $t \in \mathcal{L}(F ; H)$. Seja ainda $\left(\left(x_{j}^{1}, \ldots, x_{j}^{m_{n}}\right)\right)_{j=1}^{\infty}$ em $G_{1} \times \cdots \times G_{m_{n}}$ uma sequência multilinearmente convergente para $\left(x_{1}, \ldots, x_{m_{n}}\right)$. Vejamos que isso implica que

$$
\left(\left(B_{1}\left(x_{j}^{1}, \ldots, x_{j}^{m_{1}}\right), \ldots, B_{n}\left(x_{j}^{m_{n-1}+1}, \ldots, x_{j}^{m_{n}}\right)\right)\right)_{j=1}^{\infty} \subseteq E_{1} \times \cdots \times E_{n}
$$

converge multilinearmente para

$$
\left(B_{1}\left(x_{1}, \ldots, x_{m_{1}}\right), \ldots, B_{n}\left(x_{m_{n-1}+1}, \ldots, x_{m_{n}}\right)\right) .
$$

De fato, para toda forma $n$-linear $T \in \mathcal{L}\left(E_{1}, \ldots, E_{n}\right)$,

$$
T \circ\left(B_{1}, \ldots, B_{n}\right) \in \mathcal{L}\left(G_{1}, \ldots, G_{m_{n}}\right) .
$$


Como $\left(\left(x_{j}^{1}, \ldots, x_{j}^{m_{n}}\right)\right)_{j=1}^{\infty} \subseteq G_{1} \times \cdots \times G_{m_{n}}$ converge multilinearmente para $\left(x_{1}, \ldots, x_{m_{n}}\right)$, decorre que

$\left(T \circ\left(B_{1}, \ldots, B_{n}\right)\left(x_{j}^{1}, \ldots, x_{j}^{m_{n}}\right)\right)_{j=1}^{\infty}=\left(T \circ\left(B_{1}\left(x_{j}^{1}, \ldots, x_{j}^{m_{1}}\right), \ldots, B_{n}\left(x_{j}^{m_{n-1}+1}, \ldots, x_{j}^{m_{n}}\right)\right)\right)_{j=1}^{\infty}$

converge em norma para

$$
T \circ\left(B_{1}, \ldots, B_{n}\right)\left(x_{1}, \ldots, x_{m_{n}}\right)=T\left(B_{1}\left(x_{1}, \ldots, x_{m_{1}}\right), \ldots, B_{n}\left(x_{m_{n-1}+1}, \ldots, x_{m_{n}}\right)\right) .
$$

Como isso ocorre para toda forma $n$-linear $T$, concluímos que a sequência

$$
\left(\left(B_{1}\left(x_{j}^{1}, \ldots, x_{j}^{m_{1}}\right), \ldots, B_{n}\left(x_{j}^{m_{n-1}+1}, \ldots, x_{j}^{m_{n}}\right)\right)\right)_{j=1}^{\infty}
$$

converge multilinearmente para $\left(B_{1}\left(x_{1}, \ldots, x_{m_{1}}\right), \ldots, B_{n}\left(x_{m_{n-1}+1}, \ldots, x_{m_{n}}\right)\right)$. Combinando isso com o fato de que $A \in \mathcal{L}_{m s c}\left(E_{1}, \ldots, E_{n} ; F\right)$, implica que a sequência

$$
\left(A\left(B_{1}\left(x_{j}^{1}, \ldots, x_{j}^{m_{1}}\right), \ldots, B_{n}\left(x_{j}^{m_{n-1}+1}, \ldots, x_{j}^{m_{n}}\right)\right)\right)_{j=1}^{\infty}
$$

converge em norma para

$$
A\left(B_{1}\left(x_{1}, \ldots, x_{m_{1}}\right), \ldots, B_{n}\left(x_{m_{n-1}+1}, \ldots, x_{m_{n}}\right)\right) .
$$

A continuidade de $t$ implica que $\left(t \circ A\left(B_{1}\left(x_{j}^{1}, \ldots, x_{j}^{m_{1}}\right), \ldots, B_{n}\left(x_{j}^{m_{n-1}+1}, \ldots, x_{j}^{m_{n}}\right)\right)\right)_{j=1}^{\infty}$ converge em norma para

$$
t \circ A\left(B_{1}\left(x_{1}, \ldots, x_{m_{1}}\right), \ldots, B_{n}\left(x_{m_{n-1}+1}, \ldots, x_{m_{n}}\right)\right)
$$

Portanto a composição $t \circ A \circ\left(B_{1}, \ldots, B_{n}\right)$ pertence a $\mathcal{L}_{m s c}\left(G_{1}, \ldots, G_{m_{n}} ; H\right)$.

Falta apenas mostrar que $\mathcal{L}_{m s c}\left(E_{1}, \ldots, E_{n} ; F\right)$ é fechado em $\mathcal{L}\left(E_{1}, \ldots, E_{n} ; F\right)$ para quaisquer $n \in \mathbb{N}$ e $E_{1}, \ldots, E_{n}, F$ espaços de Banach. Para isso seja $\left(A_{j}\right)_{j=1}^{\infty}$ uma sequência em $\mathcal{L}_{m s c}\left(E_{1}, \ldots, E_{n} ; F\right)$ convergente na norma uniforme. Então existe $A \in \mathcal{L}\left(E_{1}, \ldots, E_{n} ; F\right)$ de modo que $\left(A_{j}\right)_{j=1}^{\infty}$ converge para $A$ na norma uniforme. Devemos mostrar que $A \in$ $\mathcal{L}_{m s c}\left(E_{1}, \ldots, E_{n} ; F\right)$. Consideremos então uma sequência $\left(\left(x_{k}^{1}, \ldots, x_{k}^{n}\right)\right)_{k=1}^{\infty}$ multilinearmente convergente para, digamos, $\left(x_{1}, \ldots, x_{n}\right) \in E_{1} \times \cdots \times E_{n}$. Do lema anterior podemos encontrar $K>0$ tal que $\left\|x_{k}^{1}\right\| \cdots\left\|x_{k}^{n}\right\| \leq K$ para todo $k \in \mathbb{N}$ e $\left\|x_{1}\right\| \cdots\left\|x_{n}\right\| \leq K$. Dado $\varepsilon>0$, existe $j_{0} \in \mathbb{N}$ tal que

$$
\left\|A_{j}-A\right\|<\frac{\varepsilon}{3 K}
$$

para todo $j \geq j_{0}$. Fixemos $j \geq j_{0}$. Como $A_{j} \in \mathcal{L}_{m s c}\left(E_{1}, \ldots, E_{n} ; F\right)$, existe $k_{0} \in \mathbb{N}$ tal que

$$
\left\|A_{j}\left(x_{k}^{1}, \ldots, x_{k}^{n}\right)-A_{j}\left(x_{1}, \ldots, x_{n}\right)\right\|<\frac{\varepsilon}{3},
$$


para todo $k \geq k_{0}$. Daí concluímos que

$$
\begin{aligned}
\| A\left(x_{k}^{1}, \ldots, x_{k}^{n}\right)- & A\left(x_{1}, \ldots, x_{n}\right) \| \\
\leq & \left\|A\left(x_{k}^{1}, \ldots, x_{k}^{n}\right)-A_{j}\left(x_{k}^{1}, \ldots, x_{k}^{n}\right)\right\|+\left\|A_{j}\left(x_{k}^{1}, \ldots, x_{k}^{n}\right)-A_{j}\left(x_{1}, \ldots, x_{n}\right)\right\| \\
& +\left\|A_{j}\left(x_{1}, \ldots, x_{n}\right)-A\left(x_{1}, \ldots, x_{n}\right)\right\| \\
\leq & \left\|A-A_{j}\right\| \cdot\left\|x_{k}^{1}\right\| \cdots\left\|x_{k}^{n}\right\|+\left\|A_{j}\left(x_{k}^{1}, \ldots, x_{k}^{n}\right)-A_{j}\left(x_{1}, \ldots, x_{n}\right)\right\| \\
& +\left\|A_{j}-A\right\| \cdot\left\|x_{1}\right\| \cdots\left\|x_{n}\right\| \\
< & \frac{\varepsilon}{3 K} \cdot\left\|x_{k}^{1}\right\| \cdots\left\|x_{k}^{n}\right\|+\frac{\varepsilon}{3}+\frac{\varepsilon}{3 K} \cdot\left\|x_{1}\right\| \cdots\left\|x_{n}\right\| \leq \varepsilon,
\end{aligned}
$$

para todo $k \geq k_{0}$. Portanto $\left(A\left(x_{k}^{1}, \ldots, x_{k}^{n}\right)\right)_{k=1}^{\infty}$ converge para $A\left(x_{1}, \ldots, x_{n}\right)$, ou seja $A \in \mathcal{L}_{m s c}\left(E_{1}, \ldots, E_{n} ; F\right)$. Está completa a demonstração de que $\left(\mathcal{L}_{m s c},\|\cdot\|\right)$ é um hiperideal fechado.

Em seguida consideremos o ideal fechado $\mathcal{C C}$ dos operadores completamente contínuos. Sabemos pelo Teorema 2.4.1 que $\mathcal{C C} \circ \mathcal{L}$ também é um hiper-ideal fechado. Nos perguntamos como $\mathcal{C C} \circ \mathcal{L}$ se relaciona com a recém introduzida classe $\mathcal{L}_{m s c}$. A princípio temos o seguinte:

Proposição 2.4.8. $\mathcal{C C} \circ \mathcal{L} \subseteq \mathcal{L}_{m s c}$.

Demonstração. Seja $A \in \mathcal{C} \mathcal{C} \circ \mathcal{L}\left(E_{1}, \ldots, E_{n} ; F\right)$. Para mostrar que $A \in \mathcal{L}_{m s c}\left(E_{1}, \ldots, E_{n} ; F\right)$, consideremos uma sequência $\left(\left(x_{j}^{1}, \ldots, x_{j}^{n}\right)\right)_{j=1}^{\infty}$ em $E_{1} \times \cdots \times E_{n}$ convergindo multilinearmente para $\left(x_{1}, \ldots, x_{n}\right)$. Então $\left(T\left(x_{j}^{1}, \ldots, x_{j}^{n}\right)\right)_{j=1}^{\infty}$ converge para $T\left(x_{1}, \ldots, x_{n}\right)$ em norma para toda forma $n$-linear $T \in \mathcal{L}\left(E_{1}, \ldots, E_{n}\right)$.

Agora, como $A \in \mathcal{C C} \circ \mathcal{L}\left(E_{1}, \ldots, E_{n} ; F\right)$, existem $G$ espaço de Banach, $u \in \mathcal{C C}(G ; F)$ e $B \in \mathcal{L}\left(E_{1}, \ldots, E_{n} ; G\right)$ tais que $A=u \circ B$. Consideremos $\left(B\left(x_{j}^{1}, \ldots, x_{j}^{n}\right)\right)_{j=1}^{\infty} \subseteq F$. Notemos que essa sequência converge fracamente para $B\left(x_{1}, \ldots, x_{n}\right)$, pois para todo $\varphi \in F^{\prime}$, $\varphi \circ B \in \mathcal{L}\left(E_{1}, \ldots, E_{n}\right)$ e $\left(\left(x_{j}^{1}, \ldots, x_{j}^{n}\right)\right)_{j=1}^{\infty}$ converge multilinearmente para $\left(x_{1}, \ldots, x_{n}\right)$, ou seja,

$$
\varphi \circ B\left(x_{j}^{1}, \ldots, x_{j}^{n}\right) \longrightarrow \varphi \circ B\left(x_{1}, \ldots, x_{n}\right),
$$

para todo $\varphi \in F^{\prime}$. O fato de $u$ ser completamente contínuo nos permite concluir que

$$
A\left(x_{j}^{1}, \ldots, x_{j}^{n}\right)=u \circ B\left(x_{j}^{1}, \ldots, x_{j}^{n}\right) \longrightarrow u \circ B\left(x_{1}, \ldots, x_{n}\right)=A\left(x_{1}, \ldots, x_{n}\right)
$$

em norma. Portanto $A \in \mathcal{L}_{m s c}\left(E_{1}, \ldots, E_{n} ; F\right)$.

Ainda sobre a classe $\mathcal{C C} \circ \mathcal{L}$ temos o seguinte fato, cuja demonstração foi adaptada de [13, Proposition 3.3]. 
Proposição 2.4.9. O espaço $E_{1} \widehat{\otimes}_{\pi} \cdots \widehat{\otimes}_{\pi} E_{n}$ possui a propriedade de Schur, ou seja,

$$
I d_{E_{1} \widehat{\otimes}_{\pi} \cdots \widehat{\otimes}_{\pi} E_{n}} \in \mathcal{C C}\left(E_{1} \widehat{\otimes}_{\pi} \cdots \widehat{\otimes}_{\pi} E_{n} ; E_{1} \widehat{\otimes}_{\pi} \cdots \widehat{\otimes}_{\pi} E_{n}\right),
$$

se, e somente se, $\mathcal{L}\left(E_{1}, \ldots, E_{n} ; F\right)=\mathcal{C C} \circ \mathcal{L}\left(E_{1}, \ldots, E_{n} ; F\right)$ para todo espaço de Banach F.

Demonstração. Seja $A \in \mathcal{L}\left(E_{1}, \ldots, E_{n} ; F\right)$. Pela Proposição 1.4 .6 existe um único operador $A_{L} \in \mathcal{L}\left(E_{1} \widehat{\otimes}_{\pi} \cdots \widehat{\otimes}_{\pi} E_{n} ; F\right)$ tal que $A=A_{L} \circ \delta_{n}$. Como $E_{1} \widehat{\otimes}_{\pi} \cdots \widehat{\otimes}_{\pi} E_{n}$ possui a propriedade de Schur,

$$
A_{L}=A_{L} \circ I d_{E_{1} \widehat{\otimes}_{\pi} \cdots \widehat{\otimes}_{\pi} E_{n}} \in \mathcal{C C}\left(E_{1} \widehat{\otimes}_{\pi} \cdots \widehat{\otimes}_{\pi} E_{n} ; F\right)
$$

visto que $\mathcal{C C}$ é um ideal de operadores. Então

$$
A=A_{L} \circ \delta_{n} \in \mathcal{C C} \circ \mathcal{L}\left(E_{1}, \ldots, E_{n} ; F\right) .
$$

Reciprocamente, como $\delta_{n} \in \mathcal{L}\left(E_{1}, \ldots, E_{n} ; E_{1} \widehat{\otimes}_{\pi} \cdots \widehat{\otimes}_{\pi} E_{n}\right)$ e, por hipótese,

$$
\mathcal{L}\left(E_{1}, \ldots, E_{n} ; F\right)=\mathcal{C C} \circ \mathcal{L}\left(E_{1}, \ldots, E_{n} ; F\right)
$$

para todo espaço de Banach $F$, temos pela Proposição 1.5.6, que

$$
I d_{E_{1} \widehat{\otimes}_{\pi} \cdots \widehat{\otimes}_{\pi} E_{n}}=\left(\delta_{n}\right)_{L} \in \mathcal{C C}\left(E_{1} \widehat{\otimes}_{\pi} \cdots \widehat{\otimes}_{\pi} E_{n} ; E_{1} \widehat{\otimes}_{\pi} \cdots \widehat{\otimes}_{\pi} E_{n}\right) .
$$

Portanto $E_{1} \widehat{\otimes}_{\pi} \cdots \widehat{\otimes}_{\pi} E_{n}$ tem a propriedade de Schur.

O próximo resultado relaciona as classes $\mathcal{L}_{m s c}, \mathcal{C C} \circ \mathcal{L}$ e $\mathcal{L}$ usando o fato de os espaços de saída possuirem a propriedade de Schur.

Proposição 2.4.10. Se $E_{1} \widehat{\otimes}_{\pi} \cdots \widehat{\otimes}_{\pi} E_{n}$ tem a propriedade de Schur, então

$$
\mathcal{L}_{m s c}\left(E_{1}, \ldots, E_{n} ; F\right)=\mathcal{C C} \circ \mathcal{L}\left(E_{1}, \ldots, E_{n} ; F\right)=\mathcal{L}\left(E_{1}, \ldots, E_{n} ; F\right),
$$

para todo espaço de Banach F.

Demonstração. De fato, pela Proposição 2.4.8 sabemos que

$$
\mathcal{C C} \circ \mathcal{L}\left(E_{1}, \ldots, E_{n} ; F\right) \subseteq \mathcal{L}_{m s c}\left(E_{1}, \ldots, E_{n} ; F\right)
$$

para qualquer espaço de Banach $F$. Agora, como $E_{1} \widehat{\otimes}_{\pi} \cdots \widehat{\otimes}_{\pi} E_{n}$ tem a propriedade de Schur, pela Proposição 2.4.9 temos

$$
\mathcal{C C} \circ \mathcal{L}\left(E_{1}, \ldots, E_{n} ; F\right)=\mathcal{L}\left(E_{1}, \ldots, E_{n} ; F\right)
$$


para todo espaço de Banach F. Portanto

$$
\mathcal{L}_{m s c}\left(E_{1}, \ldots, E_{n} ; F\right)=\mathcal{L}\left(E_{1}, \ldots, E_{n} ; F\right)
$$

Observação 2.4.11. A questão da preservação da propriedade de Schur pela formação do produto tensorial projetivo, isto é, se $E_{1} \widehat{\otimes}_{\pi} \cdots \widehat{\otimes}_{\pi} E_{n}$ tem a propriedade de Schur sempre que $E_{1}, \ldots, E_{n}$ têm a propriedade de Schur, está aberta há bastante tempo (veja [13]). Se resolvida positivamente esta questão, as hipóteses das Proposições 2.4.8, 2.4.9 e 2.4.10 poderão ser enfraquecidas.

\subsection{Método da desigualdade}

Introduzimos nesta seção um método para gerar hiper-ideais que, em particular, recupera como hiper-ideal um multi-ideal muito estudado (aplicações multilineares fortemente somantes).

Definição 2.5.1. Seja $0<p \leq 1$. Por $\mathcal{B} \mathcal{A N}$ denotamos a classe de todos os espaços de Banach sobre $\mathbb{K}$ e por $p-\mathcal{B} \mathcal{A N}$ a classe dos espaços $p$-Banach sobre $\mathbb{K}$. Consideremos uma correspondência

$$
\mathcal{X}: \mathcal{B A N} \longrightarrow p-\mathcal{B A N}
$$

que associa cada espaço de Banach $\left(E,\|\cdot\|_{E}\right)$ a um espaço $p$-Banach $\left(\mathcal{X}(E),\|\cdot\|_{\mathcal{X}(E)}\right)$, satisfazendo as seguintes condições:

(i) $\mathcal{X}(E)$ é um subespaço vetorial de $E^{\mathbb{N}}$ com as operações algébricas usuais;

(ii) Para cada $x \in E$ e cada $j \in \mathbb{N}$, tem-se $(0, \ldots, 0, \stackrel{j}{\underset{\downarrow}{x}}, 0, \ldots) \in \mathcal{X}(E)$ com $\|(0, \ldots, 0, x, 0, \ldots)\|_{\mathcal{X}(E)}=\|x\|_{E}$.

(iii) Para todo $u \in \mathcal{L}(E ; F)$ e toda sequência finita $\left(x_{j}\right)_{j=1}^{k}:=\left(x_{1}, \ldots, x_{k}, 0,0, \ldots\right)$ em $E$ tem-se

$$
\left\|\left(u\left(x_{j}\right)\right)_{j=1}^{k}\right\|_{\mathcal{X}(F)} \leq\|u\| \cdot\left\|\left(x_{j}\right)_{j=1}^{k}\right\|_{\mathcal{X}(E)} .
$$

Neste caso a correspondência $\mathcal{X}$ é chamada de $p$-funtor de sequências. No caso $p=1$ escrevemos simplesmente funtor de sequências.

Exemplo 2.5.2. Para $0<p \leq 1$, a correspondência

$$
(E,\|\cdot\|) \longmapsto\left(\ell_{p}(E),\|\cdot\|_{\ell_{p}(E)}\right)
$$


é um $p$-funtor de sequências, enquanto que as correspondências

$$
(E,\|\cdot\|) \longmapsto\left(c_{0}(E),\|\cdot\|_{\infty}\right) \text { e }(E,\|\cdot\|) \longmapsto\left(\ell_{\infty}(E),\|\cdot\|_{\infty}\right)
$$

são exemplos de funtores de sequências.

Definição 2.5.3 (Método da Desigualdade). Sejam $0<p, q \leq 1, \mathcal{X}$ um $p$-funtor de sequências e $\mathcal{Y}$ um $q$-funtor de sequências. Dizemos que uma aplicação $n$-linear $A \in$ $\mathcal{L}\left(E_{1}, \ldots, E_{n} ; F\right)$ é $(\mathcal{X}-\mathcal{Y})$-somante se existe uma constante $C>0$ tal que

$$
\left\|\left(A\left(x_{j}^{1}, \ldots, x_{j}^{n}\right)\right)_{j=1}^{k}\right\|_{\mathcal{Y}(F)} \leq C \cdot \sup _{T \in B_{\mathcal{L}\left(E_{1}, \ldots, E_{n}\right)}}\left\|\left(T\left(x_{j}^{1}, \ldots, x_{j}^{n}\right)\right)_{j=1}^{k}\right\|_{\mathcal{X}(\mathbb{K})},
$$

para todo $k \in \mathbb{N}$ e quaisquer sequências finitas $\left(x_{j}^{1}\right)_{j=1}^{k} \subseteq E_{1}, \ldots,\left(x_{j}^{n}\right)_{j=1}^{k} \subseteq E_{n}$. Nesse caso escrevemos $A \in(\mathcal{X}-\mathcal{Y})\left(E_{1}, \ldots, E_{n} ; F\right)$.

Além disso definimos $\|\cdot\|_{(\mathcal{X}-\mathcal{Y})}:(\mathcal{X}-\mathcal{Y})\left(E_{1}, \ldots, E_{n} ; F\right) \longrightarrow[0, \infty)$ por

$$
\|A\|_{(\mathcal{X}-\mathcal{Y})}=\inf \{C>0: C \operatorname{satisfaz}(2.8)\}
$$

Observação 2.5.4. Para dar sentido à definição acima, provemos que

$$
\sup _{T \in B_{\mathcal{L}\left(E_{1}, \ldots, E_{n}\right)}}\left\|\left(T\left(x_{j}^{1}, \ldots, x_{j}^{n}\right)\right)_{j=1}^{k}\right\|_{\mathcal{X}(\mathbb{K})}<\infty .
$$

Para isso fixemos $k \in \mathbb{N}$ e sequências finitas $\left(x_{j}^{1}\right)_{j=1}^{k} \subseteq E_{1}, \ldots,\left(x_{j}^{n}\right)_{j=1}^{k} \subseteq E_{n}$. Para toda forma $n$-linear $T \in B_{\mathcal{L}\left(E_{1}, \ldots, E_{n}\right)}$, temos

$$
\begin{aligned}
&\left\|\left(T\left(x_{j}^{1}, \ldots, x_{j}^{n}\right)\right)_{j=1}^{k}\right\|_{\mathcal{X}(\mathbb{K})}^{p}=\|\left(T\left(x_{1}^{1}, \ldots, x_{1}^{n}\right), 0, \ldots, 0, \ldots\right)+\left(0, T\left(x_{2}^{1}, \ldots, x_{2}^{n}\right), 0, \ldots, 0, \ldots\right) \\
&+\cdots+\left(0,0, \ldots, 0, T\left(x_{k}^{1}, \ldots, x_{k}^{n}\right), 0, \ldots\right) \|_{\mathcal{X}(\mathbb{K})}^{p} \\
& \leq\left\|\left(T\left(x_{1}^{1}, \ldots, x_{1}^{n}\right), 0, \ldots, 0, \ldots\right)\right\|_{\mathcal{X}(\mathbb{K})}^{p}+\left\|\left(0, T\left(x_{2}^{1}, \ldots, x_{2}^{n}\right), 0, \ldots, 0, \ldots\right)\right\|_{\mathcal{X}(\mathbb{K})}^{p} \\
&+\cdots+\left\|\left(0,0, \ldots, 0, T\left(x_{k}^{1}, \ldots, x_{k}^{n}\right), 0, \ldots\right)\right\|_{\mathcal{X}(\mathbb{K})}^{p} \\
&=\left|T\left(x_{1}^{1}, \ldots, x_{1}^{n}\right)\right|^{p}+\left|T\left(x_{2}^{1}, \ldots, x_{2}^{n}\right)\right|^{p}+\cdots+\left|T\left(x_{k}^{1}, \ldots, x_{k}^{n}\right)\right|^{p} \\
& \leq\left\|x_{1}^{1}\right\|^{p} \cdots\left\|x_{1}^{n}\right\|^{p}+\left\|x_{2}^{1}\right\|^{p} \cdots\left\|x_{2}^{n}\right\|^{p}+\cdots+\left\|x_{k}^{1}\right\|^{p} \cdots\left\|x_{k}^{n}\right\|^{p} .
\end{aligned}
$$

Como este último número não depende de $T$, tomando o supremo sobre $T \in B_{\mathcal{L}\left(E_{1}, \ldots, E_{n}\right)}$ obtemos o desejado.

Lema 2.5.5. Sejam $0<p \leq 1, \mathcal{X}$ um $p$-funtor de sequências e $n \in \mathbb{N}$. Se $\left(x_{j}^{1}\right)_{j=1}^{\infty}, \ldots,\left(x_{j}^{n}\right)_{j=1}^{\infty}$ são sequências em $E$ tais que as séries $\sum_{j=1}^{\infty} x_{j}^{1}, \ldots, \sum_{j=1}^{\infty} x_{j}^{n}$ são convergentes em E, então a série $\sum_{j=1}^{\infty}\left(x_{j}^{1}, \ldots, x_{j}^{n}, 0,0, \ldots\right)$ é convergente em $\mathcal{X}(E)$ e vale 


$$
\sum_{j=1}^{\infty}\left(x_{j}^{1}, \ldots, x_{j}^{n}, 0,0, \ldots\right)=\left(\sum_{j=1}^{\infty} x_{j}^{1}, \ldots, \sum_{j=1}^{\infty} x_{j}^{n}, 0,0, \ldots\right)
$$

Demonstração. Como $\mathcal{X}(E)$ contém as sequências finitas e $\sum_{j=1}^{\infty} x_{j}^{1}, \ldots, \sum_{j=1}^{\infty} x_{j}^{n}$ são convergentes, então

$$
\left(\sum_{j=1}^{\infty} x_{j}^{1}, \ldots, \sum_{j=1}^{\infty} x_{j}^{n}, 0,0, \ldots\right) \in \mathcal{X}(E) \text { e }\left(x_{j}^{1}, \ldots, x_{j}^{n}, 0,0, \ldots\right) \in \mathcal{X}(E),
$$

para todo $j \in \mathbb{N}$. Além disso, como trata-se de um número finito de sequências, dado $\varepsilon>0$ existe $k_{0} \in \mathbb{N}$ tal que $\left\|\sum_{j=k+1}^{\infty} x_{j}^{i}\right\|<\frac{\varepsilon}{\sqrt[p]{n}}$ para todos $k \geq k_{0}$ e $i=1, \ldots, n$. Assim

$$
\begin{aligned}
& \left\|\sum_{j=1}^{k}\left(x_{j}^{1}, \ldots, x_{j}^{n}, 0,0, \ldots\right)-\left(\sum_{j=1}^{\infty} x_{j}^{1}, \ldots, \sum_{j=1}^{\infty} x_{j}^{n}, 0,0, \ldots\right)\right\|_{\mathcal{X}(E)}^{p} \\
& =\left\|\left(\sum_{j=1}^{k} x_{j}^{1}, \ldots, \sum_{j=1}^{k} x_{j}^{n}, 0,0, \ldots\right)-\left(\sum_{j=1}^{\infty} x_{j}^{1}, \ldots, \sum_{j=1}^{\infty} x_{j}^{n}, 0,0, \ldots\right)\right\|_{\mathcal{X}(E)}^{p} \\
& =\left\|\left(\sum_{j=k+1}^{\infty} x_{j}^{1}, \ldots, \sum_{j=k+1}^{\infty} x_{j}^{n}, 0,0, \ldots\right)\right\|_{\mathcal{X}(E)}^{p} \\
& \leq\left\|\left(\sum_{j=k+1}^{\infty} x_{j}^{1}, 0, \ldots, 0, \ldots\right)\right\|_{\mathcal{X}(E)}^{p}+\cdots+\left\|\left(0, \ldots, 0, \sum_{j=k+1}^{\infty} x_{j}^{n}, 0, \ldots\right)\right\|_{\mathcal{X}(E)}^{p} \\
& =\left\|\sum_{j=k+1}^{\infty} x_{j}^{1}\right\|^{p}+\cdots+\left\|\sum_{j=k+1}^{\infty} x_{j}^{n}\right\|^{p}<\varepsilon^{p}
\end{aligned}
$$

para todo $k \geq k_{0}$. Logo $\sum_{j=1}^{\infty}\left(x_{j}^{1}, \ldots, x_{j}^{n}, 0, \ldots\right)$ converge para $\left(\sum_{j=1}^{\infty} x_{j}^{1}, \ldots, \sum_{j=1}^{\infty} x_{j}^{n}, 0,0, \ldots\right)$, o que prova o resultado.

Definição 2.5.6. Sejam $0<p, q \leq 1$. Dizemos que um $p$-funtor de sequências $\mathcal{X}$ é escalarmente dominado pelo $q$-funtor de sequências $\mathcal{Y}$ se, para toda sequência finita $\left(\lambda_{j}\right)_{j=1}^{k} \subseteq \mathbb{K}$, $k \in \mathbb{N}$, tivermos

$$
\left\|\left(\lambda_{j}\right)_{j=1}^{k}\right\|_{\mathcal{X}(\mathbb{K})} \leq\left\|\left(\lambda_{j}\right)_{j=1}^{k}\right\|_{\mathcal{Y}(\mathbb{K})} .
$$

Teorema 2.5.7. Sejam $0<p, q \leq 1$ e $\mathcal{Y}$ um q-funtor de sequências escalarmente dominado pelo p-funtor de sequências $\mathcal{X}$. Então $\left((\mathcal{X}-\mathcal{Y}),\|\cdot\|_{(\mathcal{X}-\mathcal{Y})}\right)$ é um hiper-ideal q-Banach. 
Demonstração. Provaremos usando o Critério da Série (Teorema 2.1.9).

(i) Seja $n \in \mathbb{N}$. Como $I_{n} \in B_{\mathcal{L}(n \mathbb{K})}$, para quaisquer sequências $\left(\lambda_{j}^{1}\right)_{j=1}^{k}, \ldots,\left(\lambda_{j}^{n}\right)_{j=1}^{k}$ de escalares e $k \in \mathbb{N}$, temos

$$
\begin{aligned}
\left\|\left(I_{n}\left(\lambda_{j}^{1}, \ldots, \lambda_{j}^{n}\right)\right)_{j=1}^{k}\right\|_{\mathcal{Y}(\mathbb{K})} & \leq \sup _{T \in B_{\mathcal{L}\left(n_{\mathbb{K}}\right)}}\left\|\left(T\left(\lambda_{j}^{1}, \ldots, \lambda_{j}^{n}\right)\right)_{j=1}^{k}\right\|_{\mathcal{Y}(\mathbb{K})} \\
& \leq \sup _{T \in B_{\mathcal{L}\left(n_{\mathbb{K}}\right)}}\left\|\left(T\left(\lambda_{j}^{1}, \ldots, \lambda_{j}^{n}\right)\right)_{j=1}^{k}\right\|_{\mathcal{X}(\mathbb{K})} .
\end{aligned}
$$

Então $\left\|I_{n}\right\|_{(\mathcal{X}-\mathcal{Y})} \leq 1$. Por outro lado, suponha que $C>0$ seja tal que

$$
\left\|\left(I_{n}\left(\lambda_{j}^{1}, \ldots, \lambda_{j}^{n}\right)\right)_{j=1}^{k}\right\|_{\mathcal{Y}(\mathbb{K})} \leq C \cdot \sup _{T \in B_{\mathcal{L}\left(n_{\mathbb{K}}\right)}}\left\|\left(T\left(\lambda_{j}^{1}, \ldots, \lambda_{j}^{n}\right)\right)_{j=1}^{k}\right\|_{\mathcal{X}(\mathbb{K})}
$$

para quaisquer $\left(\lambda_{j}^{1}\right)_{j=1}^{k}, \ldots,\left(\lambda_{j}^{n}\right)_{j=1}^{k} \subseteq \mathbb{K}$ e $k \in \mathbb{N}$. Em particular, para $k=1$ e $\lambda_{1}^{1}=\cdots=$ $\lambda_{1}^{n}=1$, temos

$$
\begin{aligned}
1 & =\|(1,0, \ldots, 0, \ldots)\|_{\mathcal{Y}(\mathbb{K})}=\left\|\left(I_{n}\left(\lambda_{j}^{1}, \ldots, \lambda_{j}^{n}\right)\right)_{j=1}^{1}\right\|_{\mathcal{Y}(\mathbb{K})} \\
& \leq C \cdot \sup _{T \in B_{\mathcal{L}\left(n_{\mathbb{K}}\right)}}\left\|\left(T\left(\lambda_{j}^{1}, \ldots, \lambda_{j}^{n}\right)\right)_{j=1}^{1}\right\|_{\mathcal{X}(\mathbb{K})}=C \cdot \sup _{T \in B_{\mathcal{L}\left(n_{\mathbb{K}}\right)}}\left|T\left(\lambda_{1}^{1}, \ldots, \lambda_{1}^{n}\right)\right| \leq C .
\end{aligned}
$$

Então $C \geq 1$, o que implica que $\left\|I_{n}\right\|_{(\mathcal{X}-\mathcal{Y})} \geq 1$.

(ii) Seja $\left(A_{i}\right)_{i=1}^{\infty} \subseteq(\mathcal{X}-\mathcal{Y})\left(E_{1}, \ldots, E_{n} ; F\right)$ tal que $\sum_{i=1}^{\infty}\left\|A_{i}\right\|_{(\mathcal{X}-\mathcal{Y})}^{q}<\infty$. Queremos mostrar que $A:=\sum_{i=1}^{\infty} A_{i} \in(\mathcal{X}-\mathcal{Y})\left(E_{1}, \ldots, E_{n} ; F\right)$ e $\|A\|_{(\mathcal{X}-\mathcal{Y})}^{q} \leq \sum_{i=1}^{\infty}\left\|A_{i}\right\|_{(\mathcal{X}-\mathcal{Y})}^{q}$. Note que, para toda $B \in(\mathcal{X}-\mathcal{Y})\left(E_{1}, \ldots, E_{n} ; F\right)$, temos

$$
\begin{aligned}
\left\|B\left(x_{1}, \ldots, x_{n}\right)\right\|_{F} & =\left\|\left(B\left(x_{1}, \ldots, x_{n}\right), 0, \ldots, 0, \ldots\right)\right\|_{\mathcal{Y}(F)} \\
& \leq C \cdot \sup _{T \in B_{\mathcal{L}\left(E_{1}, \ldots, E_{n}\right)}}\left\|\left(T\left(x_{1}, \ldots, x_{n}\right), 0, \ldots, 0, \ldots\right)\right\|_{\mathcal{X}(\mathbb{K})} \\
& =C \cdot \sup _{T \in B_{\mathcal{L}\left(E_{1}, \ldots, E_{n}\right)}}\left|T\left(x_{1}, \ldots, x_{n}\right)\right| \\
& \leq C \cdot\left\|x_{1}\right\| \cdots\left\|x_{n}\right\| .
\end{aligned}
$$

Então $\|B\| \leq C$ e portanto $\|B\| \leq\|B\|_{(\mathcal{X}-\mathcal{Y})}$. Com isso faz sentido definir $A:=\sum_{i=1}^{\infty} A_{i}$, visto que $\sum_{i=1}^{\infty}\left\|A_{i}\right\|^{q} \leq \sum_{i=1}^{\infty}\left\|A_{i}\right\|_{(\mathcal{X}-\mathcal{Y})}^{q}<\infty$ e $0<q \leq 1$. Além disso, 


$$
\begin{aligned}
&\left\|\left(A\left(x_{j}^{1}, \ldots, x_{j}^{n}\right)\right)_{j=1}^{k}\right\|_{\mathcal{Y}(F)}^{q}=\left\|\left(\sum_{i=1}^{\infty} A_{i}\left(x_{j}^{1}, \ldots, x_{j}^{n}\right)\right)_{j=1}^{k}\right\|_{\mathcal{Y}(F)}^{q} \\
&=\left\|\sum_{i=1}^{\infty}\left(A_{i}\left(x_{j}^{1}, \ldots, x_{j}^{n}\right)\right)_{j=1}^{k}\right\|_{\mathcal{Y}(F)}^{q} \leq \sum_{i=1}^{\infty}\left\|\left(A_{i}\left(x_{j}^{1}, \ldots, x_{j}^{n}\right)\right)_{j=1}^{k}\right\|_{\mathcal{Y}(F)}^{q} \\
& \leq \sum_{i=1}^{\infty}\left\|A_{i}\right\|_{\mathcal{X}-\mathcal{Y}}^{q} \cdot\left(\sup _{\left.T \in B_{\mathcal{L}\left(E_{1}, \ldots, E_{n}\right)}\left\|\left(T\left(x_{j}^{1}, \ldots, x_{j}^{n}\right)\right)_{j=1}^{k}\right\|_{\mathcal{X}(\mathbb{K})}\right)^{q}}\right. \\
&=\left(\sum_{i=1}^{\infty}\left\|A_{i}\right\|_{\mathcal{X}-\mathcal{Y}}^{q}\right) \cdot\left(\sup _{T \in B_{\mathcal{L}\left(E_{1}, \ldots, E_{n}\right)}}\left\|\left(T\left(x_{j}^{1}, \ldots, x_{j}^{n}\right)\right)_{j=1}^{k}\right\|_{\mathcal{X}(\mathbb{K})}\right)^{q},
\end{aligned}
$$

onde a segunda igualdade decorre do Lema 2.5.5 e a segunda desigualdade do fato que $A_{i} \in(\mathcal{X}-\mathcal{Y})\left(E_{1}, \ldots, E_{n} ; F\right)$ para todo $i \in \mathbb{N}$. Disso segue que

$\left\|\left(A\left(x_{j}^{1}, \ldots, x_{j}^{n}\right)\right)_{j=1}^{k}\right\|_{\mathcal{Y}(F)} \leq\left(\sum_{i=1}^{\infty}\left\|A_{i}\right\|_{\mathcal{X}-\mathcal{Y}}^{q}\right)^{1 / q} \cdot\left(\sup _{T \in B_{\mathcal{L}\left(E_{1}, \ldots, E_{n}\right)}}\left\|\left(T\left(x_{j}^{1}, \ldots, x_{j}^{n}\right)\right)_{j=1}^{k}\right\|_{\mathcal{X}(\mathbb{K})}\right)$,

para quaisquer sequências finitas $\left(x_{j}^{1}\right)_{j=1}^{k} \subseteq E_{1}, \ldots,\left(x_{j}^{n}\right)_{j=1}^{k} \subseteq E_{n}$ e $k \in \mathbb{N}$. Concluímos que $A \in(\mathcal{X}-\mathcal{Y})\left(E_{1}, \ldots, E_{n} ; F\right)$ e $\|A\|_{(\mathcal{X}-\mathcal{Y})}^{q} \leq \sum_{i=1}^{\infty}\left\|A_{i}\right\|_{(\mathcal{X}-\mathcal{Y})}^{q}$.

(iii) Sejam $1 \leq m_{1}<\cdots<m_{n}$ números naturais, $A \in(\mathcal{X}-\mathcal{Y})\left(E_{1}, \ldots, E_{n} ; F\right)$, $B_{1} \in \mathcal{L}\left(G_{1}, \ldots, G_{m_{1}} ; E_{1}\right), \ldots, B_{n} \in \mathcal{L}\left(G_{m_{n-1}+1}, \ldots, G_{m_{n}} ; E_{n}\right)$ e $t \in \mathcal{L}(F ; H)$. É claro que podemos supor $B_{l} \neq 0$ para $l=1, \ldots, n$, e então

$$
\begin{aligned}
& \left\|\left(t \circ A \circ\left(B_{1}, \ldots, B_{n}\right)\left(x_{j}^{1}, \ldots, x_{j}^{n}\right)\right)_{j=1}^{k}\right\|_{\mathcal{Y}(H)} \\
& =\left\|\left(t\left(A\left(B_{1}\left(x_{j}^{1}, \ldots, x_{j}^{m_{1}}\right), \ldots, B_{n}\left(x_{j}^{m_{n-1}+1}, \ldots, x_{j}^{m_{n}}\right)\right)\right)\right)_{j=1}^{k}\right\|_{\mathcal{Y}(H)} \\
& \leq\|t\| \cdot\left\|\left(A\left(B_{1}\left(x_{j}^{1}, \ldots, x_{j}^{m_{1}}\right), \ldots, B_{n}\left(x_{j}^{m_{n-1}+1}, \ldots, x_{j}^{m_{n}}\right)\right)\right)_{j=1}^{k}\right\|_{\mathcal{Y}(F)} \\
& \leq\|t\| \cdot\|A\|_{\mathcal{X}-\mathcal{Y}} \cdot \sup _{T \in B_{\mathcal{L}\left(E_{1}, \ldots, E_{n}\right)}}\left\|\left(T\left(B_{1}\left(x_{j}^{1}, \ldots, x_{j}^{m_{1}}\right), \ldots, B_{n}\left(x_{j}^{m_{n-1}+1}, \ldots, x_{j}^{m_{n}}\right)\right)\right)_{j=1}^{k}\right\|_{\mathcal{X}(\mathbb{K})} \\
& =\|t\| \cdot\|A\|_{\mathcal{X}-\mathcal{Y}} \cdot\left\|B_{1}\right\| \cdots\left\|B_{n}\right\| \cdot \\
& \sup _{T \in B_{\mathcal{L}\left(E_{1}, \ldots, E_{n}\right)}}\left\|\left(T\left(\frac{B_{1}}{\left\|B_{1}\right\|}\left(x_{j}^{1}, \ldots, x_{j}^{m_{1}}\right), \ldots, \frac{B_{n}}{\left\|B_{n}\right\|}\left(x_{j}^{m_{n-1}+1}, \ldots, x_{j}^{m_{n}}\right)\right)\right)_{j=1}^{k}\right\|_{\mathcal{X}(\mathbb{K})} \\
& \leq\|t\| \cdot\|A\|_{\mathcal{X}-\mathcal{Y}} \cdot\left\|B_{1}\right\| \cdots\left\|B_{n}\right\|_{S \in B_{\mathcal{L}\left(G_{1}, \ldots, G_{m_{n}}\right)}}\left\|\left(S\left(x_{j}^{1}, \ldots, x_{j}^{m_{n}}\right)\right)_{j=1}^{k}\right\|_{\mathcal{X}(\mathbb{K})} .
\end{aligned}
$$

Note que usamos a condição (iii) da Definição 2.5.1 na primeira desigualdade, e que para todo $T \in B_{\mathcal{L}\left(E_{1}, \ldots, E_{n}\right)}$ temos

$$
T \circ\left(\frac{B_{1}}{\left\|B_{1}\right\|}, \ldots, \frac{B_{n}}{\left\|B_{n}\right\|}\right) \in B_{\mathcal{L}\left(G_{1}, \ldots, G_{m_{n}}\right)},
$$


na última desigualdade. Assim $t \circ A \circ\left(B_{1}, \ldots, B_{n}\right) \in(\mathcal{X}-\mathcal{Y})\left(G_{1}, \ldots, G_{m_{n}} ; H\right)$ e

$$
\left\|t \circ A \circ\left(B_{1}, \ldots, B_{n}\right)\right\|_{(\mathcal{X}-\mathcal{Y})} \leq\|t\| \cdot\|A\|_{\mathcal{X}-\mathcal{Y}} \cdot\left\|B_{1}\right\| \cdots\left\|B_{n}\right\| .
$$

Portanto $\left((\mathcal{X}-\mathcal{Y}),\|\cdot\|_{(\mathcal{X}-\mathcal{Y})}\right)$ é um hiper-ideal $q$-Banach.

Apresentamos a seguir exemplos de hiper-ideais obtidos através do método que acabamos de introduzir. Começamos descrevendo o contexto histórico do primeiro exemplo, passando por algumas definições e resultados que motivaram sua definição. Relembremos então um importante ideal de operadores lineares.

Definição 2.5.8. Seja $1 \leq p<\infty$. Um operador $u: E \longrightarrow F$ é chamado absolutamente $p$-somante se existe $C>0$ tal que

$$
\left(\sum_{i=1}^{k}\left\|u\left(x_{i}\right)\right\|^{p}\right)^{1 / p} \leq C \cdot \sup _{\varphi \in B_{E^{\prime}}}\left\{\left(\sum_{i=1}^{k}\left|\varphi\left(x_{i}\right)\right|^{p}\right)^{1 / p}\right\}
$$

para qualquer família finita de vetores $\left(x_{i}\right)_{i=1}^{k}$ em $E, k \in \mathbb{N}$. Define-se ainda

$$
\|u\|_{\pi_{p}}=\inf \{C: C \text { satisfaz }(2.9)\} .
$$

O espaço dos operadores $p$-somantes de $E$ em $F$ é denotado por $\Pi_{p}(E ; F)$. O par $\left(\Pi_{p},\|\cdot\|_{\pi_{p}}\right)$ é um dos ideais de operadores de Banach mais estudados, veja, por exemplo, [25].

Na busca por uma classe de aplicações multilineares que seja uma boa generalização multilinear dos operadores absolutamente somantes, o próprio Pietsch propôs o seguinte conceito:

Definição 2.5.9. (Pietsch [44]) Sejam $1 \leq p_{1}, \ldots, p_{n}<\infty$ e $1 / s=1 / p_{1}+\cdots+1 / p_{n}$. Uma aplicação $n$-linear $A: E_{1} \times \cdots \times E_{n} \longrightarrow F$ é dita $\left(p_{1}, \ldots, p_{n}\right)$-dominada se existe $C>0$ tal que

$$
\begin{aligned}
\left(\sum_{i=1}^{k}\left\|A\left(x_{i}^{1}, \ldots, x_{i}^{n}\right)\right\|^{s}\right)^{1 / s} \leq & C\left(\sup _{\varphi_{1} \in B_{E_{1}^{\prime}}}\left\{\left(\sum_{i=1}^{k}\left|\varphi_{1}\left(x_{i}^{1}\right)\right|^{p_{1}}\right)^{1 / p_{1}}\right\}\right) \cdots \\
& \ldots\left(\sup _{\varphi_{n} \in B_{E_{n}^{\prime}}}\left\{\left(\sum_{i=1}^{k}\left|\varphi_{n}\left(x_{i}^{n}\right)\right|^{p_{n}}\right)^{1 / p_{n}}\right\}\right),
\end{aligned}
$$

para quaisquer famílias finitas de vetores $\left(x_{i}^{l}\right)_{i=1}^{k} \subseteq E_{l}, l=1, \ldots, n, k \in \mathbb{N}$. Define-se ainda

$$
\|A\|_{d ; p_{1}, \ldots, p_{n}}=\inf \{C: C \text { satisfaz }(2.10)\} .
$$


Se $p_{1}=\cdots=p_{n}=p$ dizemos simplesmente que a aplicação é $p$-dominada e escrevemos $\|A\|_{d, p}$.

O famoso resultado a seguir relaciona o multi-ideal das aplicações $\left(p_{1}, \ldots, p_{n}\right)$-dominadas com o método da fatoração para obtenção de multi-ideais.

Proposição 2.5.10. Seja $A \in \mathcal{L}\left(E_{1}, \ldots, E_{n} ; F\right)$. São equivalentes:

1) A é $\left(p_{1}, \ldots, p_{n}\right)$-dominada;

2) Existem espaços de Banach $G_{1}, \ldots, G_{n}$, operadores lineares $u_{j} \in \Pi_{p}\left(E_{j} ; G_{j}\right), j=$ $1, \ldots, n$, e $B \in \mathcal{L}\left(G_{1}, \ldots, G_{n} ; F\right)$ tais que $A=B \circ\left(u_{1}, \ldots, u_{n}\right)$.

Além disso,

$$
\|A\|_{d ; p_{1}, \ldots, p_{n}}=\inf \left\{\|B\| \cdot\left\|u_{1}\right\|_{\pi_{p_{1}}} \cdots\left\|u_{n}\right\|_{\pi_{p_{n}}}\right\}
$$

onde o ínfimo é tomado sobre todas as fatorações na forma $A=B \circ\left(u_{1}, \ldots, u_{n}\right)$.

Esse resultado foi enunciado por Pietsch [44, Theorem 14] sem demonstração. Uma primeira demonstração, incompleta, apareceu no trabalho de Geiss [32]. Uma nova demonstração apareceu no trabalho [41] de Pérez-García (Corolario 3.23). Entretanto esta demonstração possui um equívoco. Este equívoco foi corrigido por Botelho, Pellegrino e Rueda em [11, Section 4].

O fato das aplicações dominadas formarem um multi-ideal, e portanto serem estáveis pela composição com operadores lineares à esquerda, levou D. Popa a questionar se seriam também estáveis por composições com aplicações multilineares à esquerda. O resultado é o seguinte:

Proposição 2.5.11. (D. Popa [47, Proposition 4.2(b)]) Dados $1 \leq m_{1}<\cdots<m_{n}$ números naturais e $1 \leq p_{1}, \ldots, p_{n}<\infty$, são equivalentes:

(i) Sejam $B_{1}:\left(c_{0}\right)^{m_{1}} \longrightarrow c_{0}, \ldots, B_{k}:\left(c_{0}\right)^{m_{n}-m_{n-1}} \longrightarrow c_{0}$ aplicações multilineares contínuas e $A:\left(c_{0}\right)^{n} \longrightarrow c_{0}$ uma aplicação multilinear $\left(p_{1}, \ldots, p_{n}\right)$-dominada. Então a composição $A \circ\left(B_{1}, \ldots, B_{n}\right)$ é $(\underbrace{p_{1}, \ldots, p_{1}}_{m_{1} \text { vezes }}, \ldots, \underbrace{p_{n}, \ldots, p_{n}}_{m_{n}-m_{n-1} \text { vezes }})$-dominada;

(ii) $m_{1}=1, \ldots, m_{n}=n$.

Em relação ao que nos interessa, este resultado diz que o multi-ideal das aplicações $\left(p_{1}, \ldots, p_{n}\right)$-dominadas não forma um hiper-ideal, uma vez que é estável apenas pela composição à esquerda por operadores lineares. Tendo em vista a Proposição 2.5.10, que diz que as aplicações $\left(p_{1}, \ldots, p_{n}\right)$-dominadas são um exemplo de uma classe obtida pelo 
método da fatoração para multi-ideais, isso reforça a pouca utilidade do método da fatoração no contexto de hiper-ideias.

Uma vez excluída a classe das aplicações dominadas da teoria de hiper-ideais, torna-se necessário identificar uma boa generalização multilinear do ideal dos operadores absolutamente somantes que seja um hiper-ideal. Encontramos esta classe no trabalho [26] de V. Dimant:

Definição 2.5.12. Sejam $0<p<\infty$ e $A \in \mathcal{L}\left(E_{1}, \ldots, E_{n} ; F\right)$. Dizemos que $A$ é fortemente $p$-somante se existe $C>0$ tal que

$$
\left(\sum_{i=1}^{k}\left\|A\left(x_{i}^{1}, \ldots, x_{i}^{n}\right)\right\|^{p}\right)^{1 / p} \leq C \cdot \sup _{T \in B_{\mathcal{L}\left(E_{1}, \ldots, E_{n}\right)}}\left\{\left(\sum_{i=1}^{k}\left|T\left(x_{i}^{1}, \ldots, x_{i}^{n}\right)\right|^{p}\right)^{1 / p}\right\}
$$

para qualquer família finita de vetores $\left(x_{i}^{l}\right)_{i=1}^{k} \subseteq E_{l}, l=1, \ldots, n, k \in \mathbb{N}$. Neste caso escrevemos $A \in \mathcal{L}_{s s}^{p}\left(E_{1}, \ldots, E_{n} ; F\right)$. Define-se ainda $\|\cdot\|_{s s, p}: \mathcal{L}_{s s}^{p} \longrightarrow[0, \infty)$ por

$$
\|A\|_{s s, p}=\inf \{C>0: C \text { satisfaz }(2.11)\} .
$$

É claro que o caso linear da definição acima recupera o ideal dos operadores absolutamente $p$-somantes. Provaremos que a classe das aplicações fortemente somantes é um hiper-ideal mostrando que é um caso particular do método introduzido nesta seção:

Proposição 2.5.13. Seja $0<p<\infty$. Então:

(a) $\left(\mathcal{L}_{s s}^{p},\|\cdot\|_{s s, p}\right)$ é um hiper-ideal $p$-Banach para todo $0<p<1$.

(b) $\left(\mathcal{L}_{s s}^{p},\|\cdot\|_{s s, p}\right)$ é hiper-ideal de Banach para todo $p \geq 1$.

Demonstração. Denotamos por $\ell_{p}(\cdot)$ o $p$-funtor de sequências $E \in \mathcal{B} \mathcal{A N} \mapsto \ell_{p}(E)$. Tomando $\mathcal{X}(E)=\mathcal{Y}(E)=\ell_{p}(E)$ para todo espaço de Banach $E$ na Definição 2.5.3, a classe das aplicações fortemente $p$-somantes $\mathcal{L}_{s s}^{p}$ definida acima coincide com a classe das aplicações $\left(\ell_{p}(\cdot)-\ell_{p}(\cdot)\right)$-somantes, inclusive com igualdade de normas (ou $p$-normas). $\mathrm{O}$ resultado segue do Teorema 2.5.7.

Apresentaremos a seguir mais um exemplo de hiper-ideal obtido por meio do método da desigualdade. Para isso precisamos relembrar a seguinte definição.

Definição 2.5.14. Seja $\left(x_{j}\right)_{j=1}^{\infty}$ uma sequência no espaço de Banach E. Consideremos as funções de Rademacher $\left(r_{j}\right)_{j=1}^{\infty}$, isto é,

$$
r_{j}:[0,1] \longrightarrow \mathbb{R}, r_{j}(t)=\operatorname{sign}\left[\operatorname{sen}\left(2^{j} \pi t\right)\right] .
$$


Dizemos que uma sequência $\left(x_{j}\right)_{j=1}^{\infty}$ em E é quase incondicionalmente somável se a série $\sum_{j=1}^{\infty} r_{j}(t) x_{j}$ for convergente em $E$ para quase todo $t \in[0,1]$ em relação à medida de Lebesgue em $[0,1]$. O espaço vetorial formado por tais sequências será denotado por $\operatorname{Rad}(E)$. Munimos esse espaço de sequências com a norma $\|\cdot\|_{\operatorname{Rad}(E)}: \operatorname{Rad}(E) \longrightarrow[0, \infty)$,

$$
\left\|\left(x_{j}\right)_{j=1}^{\infty}\right\|_{\operatorname{Rad}(E)}=\left(\int_{0}^{1}\left\|\sum_{j=1}^{\infty} r_{j}(t) x_{j}\right\|^{2} d t\right)^{1 / 2} .
$$

Por [25, Theorem 12.3] sabemos que esta norma está bem definida.

Observação 2.5.15. Da desigualdade de Kahane [25, 11.1] sabemos que, para todo $0<p<\infty$, se tivéssemos definido

$$
\left\|\left(x_{j}\right)_{j=1}^{\infty}\right\|_{\operatorname{Rad}(E)}=\left(\int_{0}^{1}\left\|\sum_{j=1}^{\infty} r_{j}(t) x_{j}\right\|^{p} d t\right)^{1 / p}
$$

obteríamos uma norma equivalente à norma definida anteriormente.

Exemplo 2.5.16. A correspondência $(E,\|\cdot\|) \mapsto\left(\operatorname{Rad}(E),\|\cdot\|_{\operatorname{Rad}(E)}\right)$ é um funtor de sequências. Verifiquemos as condições exigidas na definição:

(i) Decorre diretamente da definição de $\operatorname{Rad}(E)$.

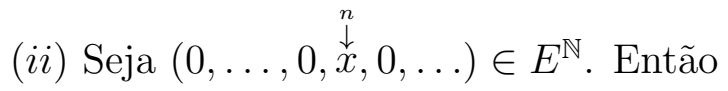

$$
\int_{0}^{1}\left\|\sum_{j=1}^{\infty} r_{j}(t) x_{j}\right\|^{2} d t=\int_{0}^{1}\left\|r_{n}(t) x\right\|^{2} d t=\|x\|^{2} .
$$

Dessa forma $(0, \ldots, 0, x, 0, \ldots) \in \operatorname{Rad}(E)$ e $\|(0, \ldots, 0, x, 0, \ldots)\|_{\operatorname{Rad}(E)}=\|x\|$.

(iii) Para toda sequência finita $\left(x_{j}\right)_{j=1}^{k} E$ e todo operador $u \in \mathcal{L}(E ; F)$, temos

$$
\begin{aligned}
\left\|\left(u\left(x_{j}\right)\right)_{j=1}^{k}\right\|_{\operatorname{Rad}(F)} & =\left(\int_{0}^{1}\left\|\sum_{j=1}^{k} r_{j}(t) u\left(x_{j}\right)\right\|^{2} d t\right)^{1 / 2}=\left(\int_{0}^{1}\left\|u\left(\sum_{j=1}^{k} r_{j}(t) x_{j}\right)\right\|^{2} d t\right)^{1 / 2} \\
& \leq\|u\| \cdot\left(\int_{0}^{1}\left\|\sum_{j=1}^{k} r_{j}(t) x_{j}\right\|^{2}\right)^{1 / 2}=\|u\| \cdot\left\|\left(x_{j}\right)_{j=1}^{k}\right\|_{\operatorname{Rad}(E) \cdot}
\end{aligned}
$$

A seguir definimos a classe em questão. Diferentemente das aplicações fortemente p-somantes, segundo nos consta essa classe é nova e ainda não foi estudada. 
Definição 2.5.17. Seja $p \geq 1$. Dizemos que uma aplicação $n$-linear $A \in \mathcal{L}\left(E_{1}, \ldots, E_{n} ; F\right)$ é fortemente quase p-somante se existe uma constante $C>0$ tal que, para todos $k \in \mathbb{N}$ e $\left(x_{j}^{1}\right)_{j=1}^{k} \subseteq E_{1}, \ldots,\left(x_{j}^{n}\right)_{j=1}^{k} \subseteq E_{n}$,

$$
\left(\int_{0}^{1}\left\|\sum_{j=1}^{k} r_{j}(t) A\left(x_{j}^{1}, \ldots, x_{j}^{n}\right)\right\|^{2} d t\right)^{1 / 2} \leq C \cdot \sup _{T \in B_{\mathcal{L}\left(E_{1}, \ldots, E_{n}\right)}}\left(\sum_{j=1}^{k}\left|\left(T\left(x_{j}^{1}, \ldots, x_{j}^{n}\right)\right)_{j=1}^{k}\right|^{p}\right)^{1 / p} .
$$

Nesse caso escrevemos $A \in \mathcal{L}_{f q s, p}\left(E_{1}, \ldots, E_{n} ; F\right)$. Definimos ainda $\|\cdot\|_{\mathcal{L}_{f q s, p}}: \mathcal{L}_{f q s, p} \longrightarrow$ $[0, \infty)$ por

$$
\|A\|_{\mathcal{L}_{f q s, p}}=\inf \{C>0: C \text { satisfaz }(2.12)\} .
$$

Proposição 2.5.18. Se $0<p \leq 2$, então $\left(\mathcal{L}_{f q s, p},\|\cdot\|_{\mathcal{L}_{f q s, p}}\right)$ é um hiper-ideal de Banach.

Demonstração. De fato, tomando $\mathcal{X}(E)=\ell_{p}(E)$ e $\mathcal{Y}(E)=\operatorname{Rad}(E)$ para todo espaço de Banach $E, \mathcal{X}$ é um $p$-funtor de sequências e $\mathcal{Y}$ é um funtor de sequências. De acordo com a Definição $2.5 .3, \mathcal{L}_{f q s, p}$ é precisamente a classe das aplicações $\left(\ell_{p}(\cdot)-\operatorname{Rad}(\cdot)\right)$-somantes. Vejamos que $\operatorname{Rad}(\cdot)$ é escalarmente dominado com respeito a $\ell_{p}(\cdot)$ : de fato, para todo $k \in \mathbb{N}$ e todos escalares $\lambda_{1}, \ldots, \lambda_{k}$,

$$
\begin{aligned}
\left\|\left(\lambda_{j}\right)_{j=1}^{k}\right\|_{\operatorname{Rad}(\mathbb{K})} & =\left(\int_{0}^{1}\left|\sum_{j=1}^{k} r_{j}(t) \lambda_{j}\right|^{2} d t\right)^{1 / 2}=\left(\sum_{j=1}^{k}\left|\lambda_{j}\right|^{2}\right)^{1 / 2} \\
& \leq\left(\sum_{j=1}^{k}\left|\lambda_{j}\right|^{p}\right)^{1 / p}=\left\|\left(\lambda_{j}\right)_{j=1}^{k}\right\|_{p} .
\end{aligned}
$$

Portanto, pelo Teorema 2.5.7 segue que $\left(\mathcal{L}_{f q s, p},\|\cdot\|_{\mathcal{L}_{f q s, p}}\right)$ é um hiper-ideal de Banach.

Observação 2.5.19. Em [50] é apresentado um método de gerar multi-ideais por meio de desigualdades, relacionado com o método para gerar hiper-ideais estudado nesta seção. Entretanto, no teorema lá enunciado [50, Theorem 3] está faltando uma hipótese, a saber, a estabilidade das sequências envolvidas por operadores lineares, ou seja, falta a condição (iii) da Definição 2.5.1.

\subsection{Método da $\mathcal{I}$-limitação}

Introduziremos nesta seção um último método para geração de hiper-ideais, que tem em comum com os métodos para geração de multi-ideais o fato de ter um ideal de operadores como ponto de partida. 
Dado um ideal de operadores $\mathcal{I}$, a noção de conjunto $\mathcal{I}$-limitado foi introduzida por Stephani em [54], e tem sido desenvolvida em vários trabalhos mais recentes, por exemplo $[4,5,33]$.

Definição 2.6.1. Seja $\mathcal{I}$ ideal de operadores. Dizemos que um subconjunto $K$ de um espaço de Banach $F$ é $\mathcal{I}$-limitado se existem um espaço de Banach $H$ e um operador $u \in \mathcal{I}(H ; F)$ tais que $K \subseteq u\left(B_{H}\right)$. A coleção dos subconjuntos $\mathcal{I}$-limitados de $F$ será denotada por $C_{\mathcal{I}}(F)$.

Em [4], Aron e Rueda utilizaram o conceito de conjunto $\mathcal{I}$-limitado para definir um ideal de polinômios homogêneos. A definição a seguir é uma adaptação da definição de Aron e Rueda para o caso de aplicações multilineares:

Definição 2.6.2. Seja $\mathcal{I}$ ideal de operadores. Dizemos que uma aplicação multilinear $A \in \mathcal{L}\left(E_{1}, \ldots, E_{n} ; F\right)$ é $\mathcal{I}$-limitada se

$$
A\left(B_{E_{1}} \times \cdots \times B_{E_{n}}\right) \in C_{\mathcal{I}}(F)
$$

ou seja, se existem um espaço de Banach $H$ e um operador $u \in \mathcal{I}(H ; F)$ tais que

$$
A\left(B_{E_{1}} \times \cdots \times B_{E_{n}}\right) \subseteq u\left(B_{H}\right) .
$$

Nesse caso escrevemos $A \in \mathcal{L}_{\langle\mathcal{I}\rangle}\left(E_{1}, \ldots, E_{n} ; F\right)$. Se $\|\cdot\|_{\mathcal{I}}$ for uma $p$-norma do ideal $\mathcal{I}$, definimos ainda $\|\cdot\|_{\mathcal{L}_{\langle\mathcal{I}\rangle}}: \mathcal{L}_{\langle\mathcal{I}\rangle} \longrightarrow[0, \infty)$ por

$$
\|A\|_{\mathcal{L}_{\langle\mathcal{I}\rangle}}=\inf \left\{\|u\|_{\mathcal{I}}: u \text { satisfaz }(2.13)\right\} .
$$

Observação 2.6.3. De acordo com a notação de [4], seria mais natural usar o símbolo $\mathcal{L}_{\mathcal{I}}$ no lugar de $\mathcal{L}_{\langle\mathcal{I}\rangle}$. Preferimos não fazer isso para não haver ambiguidade com a notação usada para outras classes, por exemplo, $\mathcal{L}_{\mathcal{F}}, \mathcal{L}_{\mathcal{N}}$, etc.

Teorema 2.6.4. Sejam $0<p \leq 1 e\left(\mathcal{I},\|\cdot\|_{\mathcal{I}}\right)$ um ideal de operadores p-normado ( $p$ Banach, respectivamente). Então $\left(\mathcal{L}_{\langle\mathcal{I}\rangle},\|\cdot\|_{\mathcal{L}_{\langle\mathcal{I}\rangle}}\right)$ é um hiper-ideal p-normado (p-Banach, respectivamente).

Demonstração. Primeiramente suponhamos que $\left(\mathcal{I},\|\cdot\|_{\mathcal{I}}\right)$ seja um ideal de operadores $p$-Banach e mostremos que $\left(\mathcal{L}_{\langle\mathcal{I}\rangle},\|\cdot\|_{\mathcal{L}_{\langle\mathcal{I}\rangle}}\right)$ é um hiper-ideal $p$-Banach usando o Critério da Série (Teorema 2.1.9).

(i) Devemos provar que $I_{n} \in \mathcal{L}_{\langle\mathcal{I}\rangle}\left(\mathbb{K}^{n}\right)$ e $\left\|I_{n}\right\|_{\mathcal{L}_{\langle\mathcal{I}\rangle}}=1$. De fato,

$$
I_{n}\left(B_{\mathbb{K}} \times \cdots \times B_{\mathbb{K}}\right) \subseteq B_{\mathbb{K}}=I d_{\mathbb{K}}\left(B_{\mathbb{K}}\right),
$$


e como $\mathcal{I}$ é ideal de operadores, $I d_{\mathbb{K}} \in \mathcal{I}(\mathbb{K} ; \mathbb{K})$. Então $I_{n}\left(B_{\mathbb{K}} \times \cdots \times B_{\mathbb{K}}\right) \in C_{\mathcal{I}}(\mathbb{K})$, ou seja, $I_{n} \in \mathcal{L}_{\langle\mathcal{I}\rangle}\left(\mathbb{K}^{n}\right)$. Além disso $\left\|I d_{\mathbb{K}}\right\|_{\mathcal{I}}=1$. Suponhamos que exista $u \in \mathcal{I}(H ; \mathbb{K})$ tal que

$$
I_{n}\left(B_{\mathbb{K}} \times \cdots \times B_{\mathbb{K}}\right) \subseteq u\left(B_{H}\right)
$$

Neste caso existe $z \in B_{H}$ tal que $u(z)=1$, logo

$$
\|u\|_{\mathcal{I}} \geq\|u\|=\sup \left\{|u(z)|: z \in B_{H}\right\} \geq 1
$$

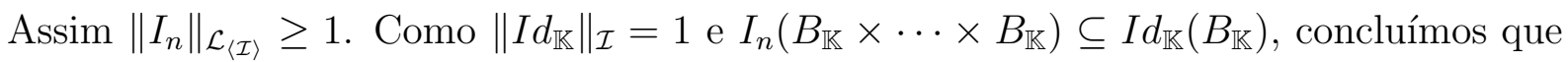
$\left\|I_{n}\right\|_{\mathcal{L}_{\langle\mathcal{I}\rangle}}=1$.

(ii) Seja $\left(A_{j}\right)_{j=1}^{\infty} \subseteq \mathcal{L}_{\langle\mathcal{I}\rangle}\left(E_{1}, \ldots, E_{n} ; F\right)$ tal que $\sum_{j=1}^{\infty}\left\|A_{j}\right\|_{\mathcal{L}_{\langle\mathcal{I}\rangle}}^{p}<\infty$. Seja $\varepsilon>0$. Para cada $j \in \mathbb{N}$, o conjunto $A_{j}\left(B_{E_{1}} \times \cdots \times B_{E_{n}}\right)$ é $\mathcal{I}$-limitado, portanto existem um espaço de Banach $H_{j}$ e um operador $u_{j} \in \mathcal{I}\left(H_{j} ; F\right)$ tais que

$$
A_{j}\left(B_{E_{1}} \times \cdots \times B_{E_{n}}\right) \subseteq u_{j}\left(B_{H_{j}}\right)
$$

e $\left\|u_{j}\right\|_{\mathcal{I}}<(1+\varepsilon)\left\|A_{j}\right\|_{\mathcal{L}_{\langle\mathcal{I}\rangle}}$. Por outro lado,

$$
\left\|A_{j}\right\| \leq\left\|u_{j}\right\| \leq\left\|u_{j}\right\|_{\mathcal{I}}<(1+\varepsilon)\left\|A_{j}\right\|_{\mathcal{L}_{\langle\mathcal{I}\rangle}} .
$$

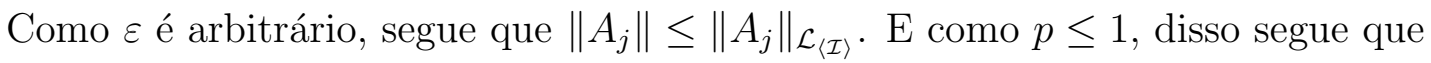

$$
\sum_{j=1}^{\infty}\left\|A_{j}\right\| \leq\left(\sum_{j=1}^{\infty}\left\|A_{j}\right\|^{p}\right)^{1 / p} \leq\left(\sum_{j=1}^{\infty}\left\|A_{j}\right\|_{\mathcal{L}_{\langle\mathcal{I}\rangle}}^{p}\right)^{1 / p}<\infty
$$

e então podemos considerar $A:=\sum_{j=1}^{\infty} A_{j} \in \mathcal{L}\left(E_{1}, \ldots, E_{n} ; F\right)$. Seja

$$
H:=\left(\bigoplus_{j=1}^{\infty} H_{j},\|\cdot\|_{\infty}\right)
$$

o espaço de Banach das sequências limitadas $\left(x_{j}\right)_{j=1}^{\infty}$, com $x_{j} \in H_{j}$ para todo $j$, munido da norma do sup. Definimos

$$
u: H \longrightarrow F, u\left(\left(x_{j}\right)_{j=1}^{\infty}\right)=\sum_{j=1}^{\infty} u_{j}\left(x_{j}\right)
$$

De

$$
\sum_{j=1}^{\infty}\left\|u_{j}\left(x_{j}\right)\right\| \leq \sum_{j=1}^{\infty}\left\|u_{j}\right\| \cdot\left\|x_{j}\right\| \leq\left(\sum_{j=1}^{\infty}\left\|u_{j}\right\|\right) \cdot\left\|\left(x_{j}\right)_{j=1}^{\infty}\right\|_{\infty}
$$


concluímos que $u$ está bem definido, é linear e contínuo. Agora, sendo $\pi_{j}: H \longrightarrow H_{j}$ a projeção canônica, consideremos

$$
v_{j}: H \longrightarrow F, v_{j}=u_{j} \circ \pi_{j}
$$

Então $v_{j} \in \mathcal{I}(H ; F)$ pela propriedade de ideal. Além disso,

$$
\sum_{j=1}^{\infty}\left\|v_{j}\right\|_{\mathcal{I}}^{p}=\sum_{j=1}^{\infty}\left\|u_{j} \circ \pi_{j}\right\|_{\mathcal{I}}^{p} \leq \sum_{j=1}^{\infty}\left\|u_{j}\right\|_{\mathcal{I}}^{p}<(1+\varepsilon)^{p} \cdot \sum_{j=1}^{\infty}\left\|A_{j}\right\|_{\mathcal{L}_{\langle\mathcal{I}\rangle}}^{p}<\infty .
$$

Por hipótese, $\mathcal{I}$ é ideal $p$-Banach e

$$
\sum_{j=1}^{\infty} v_{j}\left(\left(x_{k}\right)_{k=1}^{\infty}\right)=\sum_{n=1}^{\infty} u_{n}\left(x_{n}\right)=u\left(\left(x_{k}\right)_{k=1}^{\infty}\right)
$$

para toda sequência $\left(x_{k}\right)_{k=1}^{\infty} \in H$. Pelo Critério da Série para ideais de operadores segue que

$$
u:=\sum_{j=1}^{\infty} v_{j} \in \mathcal{I}(H ; F) \text { e }\|u\|_{\mathcal{I}}^{p} \leq(1+\varepsilon)^{p} \cdot \sum_{j=1}^{\infty}\left\|A_{j}\right\|_{\mathcal{L}_{\langle\mathcal{I}\rangle}}^{p} .
$$

Seja $y \in A\left(B_{E_{1}} \times \cdots \times B_{E_{n}}\right)$, digamos

$$
y=A\left(x_{1}, \ldots, x_{n}\right)=\sum_{j=1}^{\infty} A_{j}\left(x_{1}, \ldots, x_{n}\right)
$$

$\operatorname{com} x_{l} \in B_{E_{l}}, l=1, \ldots, n$. Para cada $j$, de

$$
A_{j}\left(B_{E_{1}} \times \cdots \times B_{E_{n}}\right) \subseteq u_{j}\left(B_{H_{j}}\right)
$$

segue que existe $z_{j} \in B_{H_{j}}$ tal que $A_{j}\left(x_{1}, \ldots, x_{n}\right)=u_{j}\left(z_{j}\right)$. E disso segue que

$$
y=\sum_{j=1}^{\infty} A_{j}\left(x_{1}, \ldots, x_{n}\right)=\sum_{j=1}^{\infty} u_{j}\left(z_{j}\right)=\sum_{j=1}^{\infty} u_{j} \circ \pi_{j}\left(\left(z_{k}\right)_{k=1}^{\infty}\right)=u\left(\left(z_{k}\right)_{k=1}^{\infty}\right),
$$

onde $\left(z_{k}\right)_{k=1}^{\infty} \in B_{H}$ pois $\left\|z_{j}\right\| \leq 1$ para todo $j$. Daí $y \in u\left(B_{H}\right)$. Assim,

$$
A\left(B_{E_{1}} \times \cdots \times B_{E_{n}}\right) \subseteq u\left(B_{H}\right),
$$

o que significa que $A\left(B_{E_{1}} \times \cdots \times B_{E_{n}}\right) \in C_{\mathcal{I}}(F)$. Portanto $A \in \mathcal{L}_{\langle\mathcal{I}\rangle}\left(E_{1}, \ldots, E_{n} ; F\right)$ e

$$
\|A\|_{\mathcal{L}_{\langle\mathcal{I}\rangle}}^{p} \leq\|u\|_{\mathcal{I}}^{p} \leq(1+\varepsilon)^{p} \cdot \sum_{j=1}^{\infty}\left\|A_{j}\right\|_{\mathcal{L}_{\langle\mathcal{I}\rangle}}^{p} .
$$


Fazendo $\varepsilon \longrightarrow 0$ segue que $\|A\|_{\mathcal{L}_{\langle\mathcal{I}\rangle}}^{p} \leq \sum_{j=1}^{\infty}\left\|A_{j}\right\|_{\mathcal{L}_{\langle\mathcal{I}\rangle}}^{p}$.

(iii) Sejam $t \in \mathcal{L}(F ; G), A \in \mathcal{L}_{\langle\mathcal{I}\rangle}\left(E_{1}, \ldots, E_{n} ; F\right)$ e $D_{1} \in \mathcal{L}\left(G_{1}, \ldots, G_{m_{1}} ; E_{1}\right), \ldots, D_{n} \in$ $\mathcal{L}\left(G_{m_{n-1}+1}, \ldots, G_{m_{n}} ; E_{n}\right)$, com $1 \leq m_{1}<\ldots<m_{n}$. Pela definição de $\mathcal{L}_{\langle\mathcal{I}\rangle}$ temos $A\left(B_{E_{1}} \times\right.$ $\left.\cdots \times B_{E_{n}}\right) \in C_{\mathcal{I}}(F)$, e portanto existe um operador $u \in \mathcal{I}(H ; F)$ tal que

$$
A\left(B_{E_{1}} \times \cdots \times B_{E_{n}}\right) \subseteq u\left(B_{H}\right) .
$$

Sem perda de generalidade podemos supor $D_{l} \neq 0$ para todo $l=1, \ldots, n$, e neste caso temos

$$
\frac{D_{l}}{\left\|D_{l}\right\|}\left(B_{G_{m_{l-1}+1}} \times \cdots \times B_{G_{m_{l}}}\right) \subseteq B_{E_{l}}
$$

Então

$$
A \circ\left(\frac{D_{1}}{\left\|D_{1}\right\|}, \ldots, \frac{D_{n}}{\left\|D_{n}\right\|}\right)\left(B_{G_{1}} \times \cdots \times B_{G_{m_{n}}}\right) \subseteq u\left(B_{H}\right)
$$

Daí obtemos

$$
t \circ A \circ\left(D_{1}, \ldots, D_{n}\right)\left(B_{G_{1}} \times \cdots \times B_{G_{m_{n}}}\right) \subseteq\left(\left\|D_{1}\right\| \cdots\left\|D_{n}\right\| t \circ u\right)\left(B_{H}\right) .
$$

Pela propriedade de ideal temos $v=\left\|D_{1}\right\| \cdots\left\|D_{n}\right\| t \circ u \in \mathcal{I}(H ; G)$, e assim

$$
t \circ A \circ\left(D_{1}, \ldots, D_{n}\right)\left(B_{G_{1}} \times \cdots \times B_{G_{m_{n}}}\right) \in C_{\mathcal{I}}(G)
$$

o que prova que

$$
t \circ A \circ\left(D_{1}, \ldots, D_{n}\right) \in \mathcal{L}_{\langle\mathcal{I}\rangle}\left(G_{1}, \ldots, G_{m_{n}} ; G\right)
$$

Além disso,

$$
\left\|t \circ A \circ\left(D_{1}, \ldots, D_{n}\right)\right\|_{\mathcal{L}_{\langle\mathcal{I}\rangle}} \leq\|v\|_{\mathcal{I}} \leq\|t\| \cdot\|u\|_{\mathcal{I}} \cdot\left\|D_{1}\right\| \cdots\left\|D_{n}\right\|
$$

Tomando o ínfimo sobre todos os operadores $u$ satisfazendo (2.14), obtemos

$$
\left\|t \circ A \circ\left(D_{1}, \ldots, D_{n}\right)\right\|_{\mathcal{L}_{\langle\mathcal{I}\rangle}} \leq\|t\| \cdot\|A\|_{\mathcal{L}_{\langle\mathcal{I}\rangle}} \cdot\left\|D_{1}\right\| \cdots\left\|D_{n}\right\|
$$

Portanto $\left(\mathcal{L}_{\langle\mathcal{I}\rangle},\|\cdot\|_{\mathcal{L}_{\langle\mathcal{I}\rangle}}\right)$ é um hiper-ideal $p$-Banach.

Para completar a demonstração, basta supor que $\left(\mathcal{I},\|\cdot\|_{\mathcal{I}}\right)$ seja um ideal $p$-normado e provar que $\left(\mathcal{L}_{\langle\mathcal{I}\rangle},\|\cdot\|_{\mathcal{L}_{\langle\mathcal{I}\rangle}}\right)$ é um hiper-ideal $p$-normado. Para isso basta provar que cada componente $\mathcal{L}_{\langle\mathcal{I}\rangle}\left(E_{1}, \ldots, E_{n} ; F\right)$ é um espaço vetorial que contém as aplicações de posto finito no qual $\|\cdot\|_{\mathcal{L}_{\langle\mathcal{I}\rangle}}$ é uma $p$-norma; pois as outras condições já foram verificadas independentemente acima.

Para isso sejam $A, B \in \mathcal{L}_{\langle\mathcal{I}\rangle}\left(E_{1}, \ldots, E_{n} ; F\right)$. Por definição, $A\left(B_{E_{1}} \times \cdots \times B_{E_{n}}\right)$ e 
$B\left(B_{E_{1}} \times \cdots \times B_{E_{n}}\right)$ são subconjuntos $\mathcal{I}$-limitados de $F$, e portanto existem espaços de Banach $H_{1}, H_{2}$ e operadores lineares $u \in \mathcal{I}\left(H_{1} ; F\right), v \in \mathcal{I}\left(H_{2} ; F\right)$ tais que

$$
A\left(B_{E_{1}} \times \cdots \times B_{E_{n}}\right) \subseteq u\left(B_{H_{1}}\right) \text { e } B\left(B_{E_{1}} \times \cdots \times B_{E_{n}}\right) \subseteq v\left(B_{H_{2}}\right) .
$$

Definimos $H:=\left(H_{1} \times H_{2},\|\cdot\|_{\infty}\right)$ e $w: H \longrightarrow F$ por

$$
w=u \circ \pi_{1}+v \circ \pi_{2},
$$

onde $\pi_{i}: H \longrightarrow H_{i}, i=1,2$, são as projeções canônicas. Da propriedade de ideal segue que $w \in \mathcal{I}(H ; F)$. Além disso

$$
\begin{aligned}
(A+B)\left(B_{E_{1}} \times \cdots \times B_{E_{n}}\right) & =A\left(B_{E_{1}} \times \cdots \times B_{E_{n}}\right)+B\left(B_{E_{1}} \times \cdots \times B_{E_{n}}\right) \\
& \subseteq u\left(B_{H_{1}}\right)+v\left(B_{H_{2}}\right)=u \circ \pi_{1}\left(B_{H}\right)+v \circ \pi_{2}\left(B_{H}\right)=w\left(B_{H}\right) .
\end{aligned}
$$

Isso prova que $(A+B)\left(B_{E_{1}} \times \cdots \times B_{E_{n}}\right)$ é um subconjunto $\mathcal{I}$-limitado de $F$, isto é, $A+B \in \mathcal{L}_{\langle\mathcal{I}\rangle}\left(E_{1}, \ldots, E_{n} ; F\right)$. Temos ainda

$$
\|A+B\|_{\mathcal{L}_{\langle\mathcal{I}\rangle}}^{p} \leq\|w\|_{\mathcal{I}}^{p} \leq\|u\|_{\mathcal{I}}^{p}+\|v\|_{\mathcal{I}}^{p}
$$

Tomando o ínfimo sobre $u$ e $v$ em $\mathcal{I}$ satisfazendo (2.15) obtemos

$$
\|A+B\|_{\mathcal{L}_{\langle\mathcal{I}\rangle}}^{p} \leq\|T\|_{\mathcal{L}_{\langle\mathcal{I}\rangle}}^{p}+\|S\|_{\mathcal{L}_{\langle\mathcal{I}\rangle}}^{p}
$$

Para todo $\lambda \in \mathbb{K}$,

$$
(\lambda A)\left(B_{E_{1}} \times \cdots \times B_{E_{n}}\right) \subseteq(\lambda u)\left(B_{H_{1}}\right),
$$

e $\lambda u \in \mathcal{I}\left(H_{1} ; F\right)$. Então $(\lambda A)\left(B_{E_{1}} \times \cdots \times B_{E_{n}}\right)$ é um subconjunto $\mathcal{I}$-limitado de $F$, ou seja, $(\lambda A) \in \mathcal{L}_{\langle\mathcal{I}\rangle}\left(E_{1}, \ldots, E_{n} ; F\right)$ e

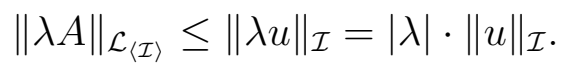

Tomando o ínfimo sobre todos os operadores $u$ satisfazendo (2.15) segue que $\|\lambda A\|_{\mathcal{L}_{\langle\mathcal{I}\rangle}} \leq$ $|\lambda| \cdot\|A\|_{\mathcal{L}_{\langle\mathcal{I}\rangle}}$. É claro que basta considerar o caso em que $\lambda \neq 0$, e neste caso temos

$$
|\lambda| \cdot\|A\|_{\mathcal{L}_{\langle\mathcal{I}\rangle}}=|\lambda| \cdot\left\|\frac{1}{\lambda} \cdot \lambda A\right\|_{\mathcal{L}_{\langle\mathcal{I}\rangle}} \leq|\lambda| \cdot \frac{1}{|\lambda|}\|\lambda A\|_{\mathcal{L}_{\langle\mathcal{I}\rangle}}=\|\lambda A\|_{\mathcal{L}_{\langle\mathcal{I}\rangle}} .
$$

Portanto $\|\lambda A\|_{\mathcal{L}_{\langle\mathcal{I}\rangle}}=|\lambda| \cdot\|A\|_{\mathcal{L}_{\langle\mathcal{I}\rangle}}$.

Provemos agora que $\mathcal{L}_{\langle\mathcal{I}\rangle}$ contém as aplicações multilineares de posto finito. Dados $T \in \mathcal{L}\left(E_{1}, \ldots, E_{n}\right)$ e $y \in F$,

$$
T \otimes y\left(B_{E_{1}} \times \cdots \times B_{E_{n}}\right) \subseteq(\|T\| I d \otimes y)\left(B_{\mathbb{K}}\right),
$$


com $\|T\| I d_{\mathbb{K}} \otimes y \in \mathcal{I}(\mathbb{K} ; F)$, uma vez que se trata de um operador linear de posto finito. Então

$$
T \otimes y\left(B_{E_{1}} \times \cdots \times B_{E_{n}}\right) \in C_{\mathcal{I}}(F)
$$

e assim $T \otimes y \in \mathcal{L}_{\langle\mathcal{I}\rangle}\left(E_{1}, \ldots, E_{n} ; F\right)$. Como as aplicações de tipo finito são somas finitas de aplicações da forma $T \otimes y$ e $\mathcal{L}_{\langle\mathcal{I}\rangle}\left(E_{1}, \ldots, E_{n} ; F\right)$ é um espaço vetorial, decorre que $\mathcal{L}_{\langle\mathcal{I}\rangle}\left(E_{1}, \ldots, E_{n} ; F\right)$ contém as aplicações de posto finito.

Para o axioma de norma que ainda falta, note que se $\|A\|_{\mathcal{L}_{\langle\mathcal{I}\rangle}}=0$, então dado $\varepsilon>0$ existe $u \in \mathcal{I}(H ; F)$ tal que

$$
A\left(B_{E_{1}} \times \cdots \times B_{E_{n}}\right) \subseteq u\left(B_{H}\right)
$$

e $\|u\|_{\mathcal{I}}<\varepsilon$. Dessa inclusão segue que

$$
\|A\| \leq\|u\| \leq\|u\|_{\mathcal{I}}<\varepsilon
$$

Dessa forma $\|A\|<\varepsilon$ para todo $\varepsilon>0$, ou seja, $A=0$. Com isso concluímos que $\|\cdot\|_{\mathcal{L}_{\langle\mathcal{I}\rangle}}$ é uma $p$-norma.

Para ilustrar este método consideramos a classe de aplicações multilineares $p$-compactas, que são definidas a seguir como uma adaptação imediata do caso polinomial estudado em Aron e Rueda [3].

Definição 2.6.5. Sejam $p, q \geq 1$ tais que $\frac{1}{p}+\frac{1}{q}=1$. Uma aplicação $n$-linear $A \in$ $\mathcal{L}\left(E_{1}, \ldots, E_{n} ; F\right)$ é dita $p$-compacta se existe uma sequência $\left(x_{j}\right)_{j=1}^{\infty} \in \ell_{p}(F)$ tal que

$$
A\left(B_{E_{1}} \times \cdots \times B_{E_{n}}\right) \subseteq\left\{\sum_{j=1}^{\infty} \lambda_{j} x_{j}:\left(\lambda_{j}\right)_{j=1}^{\infty} \in B_{\ell_{q}}\right\}
$$

Neste caso escrevemos $A \in \mathcal{L}_{\mathcal{K}_{p}}\left(E_{1}, \ldots, E_{n} ; F\right)$. Em particular, no caso $n=1$ denotamos o ideal dos operadores lineares $p$-compactos por $\mathcal{K}_{p}(E ; F)$. Definimos ainda $\|\cdot\|_{\mathcal{L}_{\mathcal{K}_{p}}}: \mathcal{L}_{\mathcal{K}_{p}} \longrightarrow$ $[0, \infty)$ por

$$
\|A\|_{\mathcal{L}_{\mathcal{K}_{p}}}=\inf \left\{\left\|\left(x_{j}\right)_{j=1}^{\infty}\right\|_{p}:\left(x_{j}\right)_{j=1}^{\infty} \operatorname{satisfaz}(2.16)\right\}
$$

Proposição 2.6.6. Para todo $p \geq 1, \mathcal{L}_{\mathcal{K}_{p}}=\mathcal{L}_{\left\langle\mathcal{K}_{p}\right\rangle}$ e $\|\cdot\|_{\mathcal{L}_{\mathcal{K}_{p}}}=\|\cdot\|_{\mathcal{L}_{\left\langle\mathcal{K}_{p}\right\rangle}}$. Em particular a classe das aplicações multilineares p-compactas é um hiper-ideal de Banach.

Demonstração. Dada $A \in \mathcal{L}_{\left\langle\mathcal{K}_{p}\right\rangle}\left(E_{1}, \ldots, E_{n} ; F\right)$, existem um espaço de Banach $H$ e um operador $p$-compacto $u \in \mathcal{K}_{p}(H ; F)$ tal que $A\left(B_{E_{1}} \times \cdots \times B_{E_{n}}\right) \subseteq u\left(B_{H}\right)$. Por outro lado, 
pela definição de operador $p$-compacto (caso $n=1$ da definição anterior), existe uma sequência $\left(x_{j}\right)_{j=1}^{\infty} \in \ell_{p}(F)$ tal que

$$
u\left(B_{H}\right) \subseteq\left\{\sum_{j=1}^{\infty} \lambda_{j} x_{j}:\left(\lambda_{j}\right)_{j=1}^{\infty} \in B_{\ell_{q}}\right\} .
$$

Com isso, obtemos uma sequência $\left(x_{j}\right)_{j=1}^{\infty} \in \ell_{p}(F)$ tal que

$$
A\left(B_{E_{1}} \times \cdots \times B_{E_{n}}\right) \subseteq\left\{\sum_{j=1}^{\infty} \lambda_{j} x_{j}:\left(\lambda_{j}\right)_{j=1}^{\infty} \in B_{\ell_{q}}\right\} .
$$

Portanto $A \in \mathcal{L}_{\mathcal{K}_{p}}\left({ }^{n} E_{1}, \ldots, E_{n} ; F\right)$, o que prova a inclusão $\mathcal{L}_{\left\langle\mathcal{K}_{p}\right\rangle} \subseteq \mathcal{L}_{\mathcal{K}_{p}}$, e $\|A\|_{\mathcal{L}_{\mathcal{K}_{p}}} \leq$ $\left\|\left(x_{j}\right)_{j=1}^{\infty}\right\|_{p}$. Tomando o ínfimo sobre todas as sequências $\left(x_{j}\right)_{j=1}^{\infty} \in \ell_{p}(F)$ satisfazendo (2.17) obtemos $\|A\|_{\mathcal{L}_{\mathcal{K}_{p}}} \leq\|u\|_{\mathcal{K}_{p}}$. Disso segue que,

$$
\|A\|_{\mathcal{L}_{\mathcal{K}_{p}}} \leq \inf \left\{\|u\|_{\mathcal{K}_{p}}: u \operatorname{satisfaz}(2.13)\right\}=\|A\|_{\mathcal{L}_{\left\langle\mathcal{K}_{p}\right\rangle}},
$$

o que prova uma das desigualdades desejadas.

Por outro lado, dada $A \in \mathcal{L}_{\mathcal{K}_{p}}\left(E_{1}, \ldots, E_{n} ; F\right)$, existe uma sequência $\left(x_{j}\right)_{j=1}^{\infty} \in \ell_{p}(F)$ tal que $A\left(B_{E_{1}} \times \cdots \times B_{E_{n}}\right) \subseteq\left\{\sum_{j=1}^{\infty} \lambda_{j} x_{j}:\left(\lambda_{j}\right)_{j=1}^{\infty} \in B_{\ell_{q}}\right\}$. Defina $u: \ell_{q} \longrightarrow F$ por

$$
u\left(\left(\lambda_{j}\right)_{j=1}^{\infty}\right)=\sum_{j=1}^{\infty} \lambda_{j} x_{j}
$$

O operador $u$ está bem definido devido à desigualdade de Hölder, é claramente linear e, por definição, é $p$-compacto. De

$$
A\left(B_{E_{1}} \times \cdots \times B_{E_{n}}\right) \subseteq\left\{\sum_{j=1}^{\infty} \lambda_{j} x_{j}:\left(\lambda_{j}\right)_{j=1}^{\infty} \in B_{\ell_{q}}\right\}=u\left(B_{\ell_{q}}\right)
$$

segue que $A \in \mathcal{L}_{\left\langle\mathcal{K}_{p}\right\rangle}\left(E_{1}, \ldots, E_{n} ; F\right)$, o que prova a inclusão $\mathcal{L}_{\mathcal{K}_{p}} \subseteq \mathcal{L}_{\left\langle\mathcal{K}_{p}\right\rangle}$, e

$$
\|A\|_{\mathcal{L}_{\left\langle\mathcal{K}_{p}\right\rangle}} \leq\|u\|_{\mathcal{K}_{p}} \leq\left\|\left(x_{j}\right)_{j=1}^{\infty}\right\|_{p}
$$

Tomando o ínfimo sobre todas as sequências $\left(x_{j}\right)_{j=1}^{\infty}$ que satisfazem as condições da definição de $\mathcal{L}_{\mathcal{K}_{p}}$ concluímos que $\|A\|_{\mathcal{L}_{\left\langle\mathcal{K}_{p}\right\rangle}} \leq\|A\|_{\mathcal{L}_{\mathcal{K}_{p}}}$.

Observação 2.6.7. Devido ao Lema de Grothendieck (veja, por exemplo, [36, Lemma 3.4.31] ou [52, Teorema 2.1.4], no caso particular em que $p=\infty$ e $\left(\lambda_{j}\right)_{j=1}^{\infty} \in B_{\ell_{1}}$ recuperamos uma vez mais o caso das aplicações multilineares (e também operadores lineares) compactas, ou seja $\mathcal{L}_{\mathcal{K}}=\mathcal{L}_{\langle\mathcal{K}\rangle}$. Com as devidas adaptações também é possível mostrar que $\mathcal{L}_{\mathcal{W}}=\mathcal{L}_{\langle\mathcal{W}\rangle}$, o que nos fornece uma outra demonstração de que a classe das aplicações multilineares fracamente compactas é um hiper-ideal fechado. 


\section{Capítulo 3}

\section{Hiper-ideais de Polinômios Homogêneos}

Assim como a teoria de multi-ideais caminha pari passu com a teoria de ideais de polinômios homogêneos, exploraremos neste capítulo a contrapartida polinomial do conceito de hiper-ideais de aplicações multilineares. Além de seu interesse formal, o estudo de ideais de polinômios homogêneos provou sua utilidade quando combinado com outras estruturas, por exemplo tipos de holomorfia (veja, por exemplo, [6, 8, 28, 29]) ou sequências coerentes (veja, por exemplo, [17, 18, 19, 39]).

Como é de se esperar, muitas vezes os resultados sobre hiper-ideais de polinômios têm demonstrações análogas às dos resultados correspondentes para hiper-ideais. Para evitar repetições desnecessárias, nesses casos omitimos os detalhes das demonstrações e fazemos menção à demonstração análoga no caso de hiper-ideais.

\subsection{Definição e propriedades básicas}

Apresentamos nesta seção a definição de hiper-ideais ( $p$-normados, $p$-Banach) de polinômios homogêneos e enunciamos suas primeiras propriedades. Deve ser destacado o fato de que uma diferença importante com ideais de polinômios aparece já na definição.

Definição 3.1.1. Um hiper-ideal de polinômios homogêneos, ou simplesmente hiper-ideal de polinômios, é uma subclasse $\mathcal{Q}$ da classe dos polinômios homogêneos contínuos entre espaços de Banach tal que, para todo $n \in \mathbb{N}$ e quaisquer espaços de Banach $E$ e $F$, a 
componente

$$
\mathcal{Q}\left({ }^{n} E ; F\right):=\mathcal{P}\left({ }^{n} E ; F\right) \cap \mathcal{Q}
$$

satisfaz as seguintes condições:

(1) $\mathcal{Q}\left({ }^{n} E ; F\right)$ é um subespaço vetorial de $\mathcal{P}\left({ }^{n} E ; F\right)$ que contém os polinômios de tipo finito;

(2) A propriedade de hiper-ideal: se $P \in \mathcal{Q}\left({ }^{n} E ; F\right), Q \in \mathcal{P}\left({ }^{m} G ; E\right)$ e $t \in \mathcal{L}(F ; H)$, então $t \circ P \circ Q \in \mathcal{Q}\left({ }^{m n} G ; H\right)$.

Se existe uma função $\|\cdot\|_{\mathcal{Q}}: \mathcal{Q} \longrightarrow[0, \infty)$ tal que:

(a) A função $\|\cdot\|_{\mathcal{Q}}$ restrita a $\mathcal{Q}\left({ }^{n} E ; F\right)$ é uma $p$-norma para quaisquer espaços de Banach $E$ e $F$ e todo $n \in \mathbb{N}$;

(b) $\left\|P: \mathbb{K} \longrightarrow \mathbb{K}, P(\lambda)=\lambda^{n}\right\|_{\mathcal{Q}}=1$ para todo $n \in \mathbb{N}$;

(c) Se $Q \in \mathcal{P}\left({ }^{m} G ; E\right), P \in \mathcal{Q}\left({ }^{n} E ; F\right)$ e $t \in \mathcal{L}(F ; H)$, então

$$
\|t \circ P \circ Q\|_{\mathcal{Q}} \leq C_{m, n} \cdot\|t\| \cdot\|P\|_{\mathcal{Q}} \cdot\|Q\|^{n} \text {, onde } C_{m, n}=\left(\frac{m^{m}}{m !}\right)^{n} ;
$$

então $\left(\mathcal{Q},\|\cdot\|_{\mathcal{Q}}\right)$ é um hiper-ideal p-normado de polinômios. Mais ainda, se todas as componentes $\mathcal{Q}\left({ }^{n} E ; F\right)$ são espaços completos relativamente a $\|\cdot\|_{\mathcal{Q}}$, então dizemos que $\left(\mathcal{Q},\|\cdot\|_{\mathcal{Q}}\right)$ é um hiper-ideal $p$-Banach de polinômios. No caso $p=1$ escrevemos simplesmente hiper-ideal normado de polinômios e hiper-ideal de Banach de polinômios. No caso em que é completo com a norma uniforme escrevemos hiper-ideal fechado de polinômios.

Observação 3.1.2. $(i)$ Decorre da definição acima que todo hiper-ideal $p$-normado ( $p$ Banach) de polinômios $\left(\mathcal{Q},\|\cdot\|_{\mathcal{Q}}\right)$ é um ideal $p$-normado ( $p$-Banach) de polinômios.

De fato, basta tomar $Q \in \mathcal{P}\left({ }^{1} G ; E\right)=\mathcal{L}(G ; E)$ e observar que $C_{1, n}=1$ para todo $n \in \mathbb{N}$. A justificativa é analoga à da Observação 2.1.3(i).

(ii) Se $\mathcal{Q}$ satisfaz (1) e (2), então $(\mathcal{Q},\|\cdot\|)$ é um hiper-ideal normado de polinômios, onde $\|\cdot\|$ é a norma uniforme (vide Proposição 1.3.2).

(iii) A constante $C_{m, n}$ da condição (c) marca uma diferença importante com o conceito de ideais de polinômios. Como visto no item $(i)$ desta observação, ela não impede que hiperideais $p$-normados de polinômios sejam casos particulares de ideais $p$-normados de polinômios. Entretanto, esta constante se impõe, ao fazermos a composição à esquerda com polinômios de grau de homogeneidade maior ou igual a 2, como condição necessária para a compatibilização das teorias de hiper-ideais de polinômios homogêneos e de aplicações multilineares. O momento exato dessa imposição pode ser claramente identificado na demonstração do item $(i i)(c)$ da Proposição 3.3.2. É claro que esta constante está intimamente associada à constante de polarização, ou seja, à relação da norma de um polinômio com a norma da sua aplicação multilinear associada. 
De acordo com o item $(i i)$ da observação acima e a Definição 2.1.4, consideremos a: Definição 3.1.3. Seja $\mathcal{Q}$ um hiper-ideal de polinômios. Definimos o fecho $\overline{\mathcal{Q}}$ de $\mathcal{Q}$ por

$$
\overline{\mathcal{Q}}\left({ }^{n} E ; F\right):=\overline{\mathcal{Q}\left({ }^{n} E ; F\right)}{ }^{\|\cdot\|},
$$

para todos $n \in \mathbb{N}$ e espaços de Banach $E$ e $F$.

Assim, $\mathcal{Q}$ é fechado se, e somente se, $\overline{\mathcal{Q}}=\mathcal{Q}$, ou seja, se $\mathcal{Q}\left({ }^{n} E ; F\right)$ é um subespaço fechado de $\mathcal{P}\left({ }^{n} E ; F\right)$ em relação a norma uniforme para todo $n \in \mathbb{N}$ e quaisquer espaços de Banach $E$ e $F$.

Inspirados pela Proposição 2.1.5, temos o seguinte resultado, cuja demonstração segue o argumento da demonstração da mesma.

Proposição 3.1.4. Se $\mathcal{Q}$ é um hiper-ideal de polinômios, então $(\overline{\mathcal{Q}},\|\cdot\|)$ é um hiper-ideal fechado de polinômios. Além disso, se $\mathcal{Q}^{\prime}$ é um hiper-ideal fechado de polinômios que contém $\mathcal{Q}$, então $\overline{\mathcal{Q}} \subseteq \mathcal{Q}^{\prime}$, ou seja, $\overline{\mathcal{Q}}$ é o menor hiper-ideal fechado de polinômios que contém $\mathcal{Q}$.

Listamos a seguir as propriedades gerais de hiper-ideais de polinômios.

Proposição 3.1.5. Seja $\left(\mathcal{Q},\|\cdot\|_{\mathcal{Q}}\right)$ um hiper-ideal p-normado de polinômios. Então

$$
\|\cdot\| \leq\|\cdot\|_{\mathcal{Q}}
$$

Demonstração. Segue de argumento análogo ao usado na demonstração da Proposição 2.1.6.

Proposição 3.1.6. Sejam $\left(\mathcal{Q},\|\cdot\|_{\mathcal{Q}}\right)$ um hiper-ideal p-normado de polinômios, $Q \in$ $\mathcal{P}\left({ }^{n} E\right), y \in F$ e $m \in \mathbb{N}$. Consideremos o polinômio nm-homogêneo $Q^{m} \otimes y: E \longrightarrow F$ dado por $Q^{m} \otimes y(x)=Q(x)^{m} \cdot y$. Então $Q^{m} \otimes y \in \mathcal{Q}\left({ }^{n m} E ; F\right) e$

$$
\left\|Q^{m} \otimes y\right\|=\|Q\|^{m} \cdot\|y\| \leq\left\|Q^{m} \otimes y\right\|_{\mathcal{Q}} \leq C_{m, n} \cdot\|Q\|^{m} \cdot\|y\| .
$$

Em particular, se $Q$ é linear então vale a igualdade.

Demonstração. Provaremos em breve (veja Proposição 3.2.2) que todo hiper-ideal de polinômios contém os polinômios homogêneos de posto finito, portanto $Q^{m} \otimes y \in \mathcal{Q}\left({ }^{n m} E ; F\right)$. Pela definição da norma uniforme sabemos que

$$
\left\|Q^{m} \otimes y(x)\right\|=|Q(x)|^{m} \cdot\|y\|
$$


para todo $x \in E$, donde decorre imediatamente que

$$
\left\|Q^{m} \otimes y\right\|=\|Q\|^{m} \cdot\|y\|
$$

Por outro lado, escrevendo

$$
Q^{m} \otimes y=(1 \otimes y) \circ \widehat{I_{m}} \circ Q
$$

segue que

$$
\left\|Q^{m} \otimes y\right\| \leq C_{m, n} \cdot\|1 \otimes y\| \cdot\left\|\widehat{I_{m}}\right\|_{\mathcal{Q}} \cdot\|Q\|^{m}=C_{m, n} \cdot\|1 \otimes y\| \cdot\|Q\|^{m}
$$

No caso em que $Q$ é linear temos $C_{m, n}=1$, e a igualdade segue.

Teorema 3.1.7 (Critério da Série). Sejam $0<p \leq 1$ e $\left(\mathcal{Q},\|\cdot\|_{\mathcal{Q}}\right)$ subclasse da classe dos polinômios homogêneos entre espaços de Banach munida de uma correspondência $\|\cdot\|_{\mathcal{Q}}: \mathcal{Q} \longrightarrow[0, \infty)$. Então $\left(\mathcal{Q},\|\cdot\|_{\mathcal{Q}}\right)$ é um hiper-ideal p-Banach de polinômios se, $e$ somente se, as seguintes condições estão satisfeitas:

(i) $\widehat{I_{n}} \in \mathcal{Q}\left({ }^{n} \mathbb{K}\right)$ e $\left\|\widehat{I_{n}}\right\|_{\mathcal{Q}}=1$ para todo $n \in \mathbb{N} ;$

(ii) $S e\left(P_{j}\right)_{j=1}^{\infty} \subseteq \mathcal{Q}\left({ }^{n} E ; F\right)$ é tal que $\sum_{j=1}^{\infty}\left\|P_{j}\right\|_{\mathcal{Q}}^{p}<\infty$, então $P:=\sum_{j=1}^{\infty} P_{j} \in \mathcal{Q}\left({ }^{n} E ; F\right) e$ $\|P\|_{\mathcal{Q}}^{p} \leq \sum_{j=1}^{\infty}\left\|P_{j}\right\|_{\mathcal{Q}}^{p}$

(iii) Se $P \in \mathcal{Q}\left({ }^{n} E ; F\right), Q \in \mathcal{P}\left({ }^{m} G ; F\right)$ e $t \in \mathcal{L}(F ; H)$, então $t \circ P \circ Q \in \mathcal{Q}\left({ }^{m n} G ; H\right) e$

$$
\|t \circ P \circ Q\|_{\mathcal{Q}} \leq C_{m, n} \cdot\|t\| \cdot\|P\|_{\mathcal{Q}} \cdot\|Q\|^{n}
$$

Demonstração. Análoga à do Teorema 2.1.9.

Proposição 3.1.8. Sejam $0<p, q \leq 1,\left(\mathcal{Q},\|\cdot\|_{\mathcal{Q}}\right)$ um hiper-ideal p-Banach de polinômios $e\left(\mathcal{R},\|\cdot\|_{\mathcal{R}}\right)$ um hiper-ideal $q$-Banach de polinômios. Se $\mathcal{Q} \subseteq \mathcal{R}$, então para cada $n \in \mathbb{N}$ existe uma constante $C_{n}>0$ tal que

$$
\|P\|_{\mathcal{R}} \leq C_{n}\|P\|_{\mathcal{Q}}
$$

para todos espaços de Banach $E, F, n \in \mathbb{N}$ e $P \in \mathcal{Q}\left({ }^{n} E ; F\right)$.

Demonstração. Análoga à da Proposição 2.1.10. 


\subsection{Exemplos: polinômios de tipo finito, de posto finito e hiper-nucleares}

Primeiramente notemos que a classe dos polinômios de tipo finito não é um hiper-ideal de polinômios.

Exemplo 3.2.1. Seja $P: \ell_{2} \longrightarrow \mathbb{K}$ dado por $P(x)=\sum_{j=1}^{\infty} x_{j}^{2}$, onde $x=\left(x_{j}\right)_{j=1}^{\infty} \in \ell_{2}$. Então $P \in \mathcal{P}\left({ }^{2} \ell_{2}\right)$, mas $P$ não é de tipo finito. Isso pode ser verificado usando o mesmo argumento do Exemplo 2.2.1 (notemos ainda que $P=\widehat{T}$, onde $T$ é a aplicação bilinear definida naquele exemplo). Então $\mathcal{P}_{f}$ não pode ser hiper-ideal de polinômios, pois caso contrário teríamos $P=I d_{\mathbb{K}} \circ P \in \mathcal{P}_{f}\left({ }^{2} \ell_{2}\right)$, o que não ocorre.

Como antes, excluídos os polinômios de tipo finito, os polinômios de posto finito formam o menor hiper-ideal de polinômios:

Teorema 3.2.2. A classe $\mathcal{P}_{\mathcal{F}}$ dos polinômios homogêneos de posto finito é o menor hiperideal de polinômios. Desse modo, se $\left(\mathcal{Q},\|\cdot\|_{\mathcal{Q}}\right)$ é um hiper-ideal p-normado de polinômios, então $\mathcal{P}_{\mathcal{F}} \subseteq \mathcal{Q}$ e $\|\cdot\| \leq\|\cdot\|_{\mathcal{Q}}$.

Demonstração. Para provar que $\mathcal{P}_{\mathcal{F}}$ é um hiper-ideal de polinômios, usando o mesmo raciocínio do Teorema 2.2.2, basta provar que

$$
t \circ P \circ Q \in \mathcal{P}_{\mathcal{F}}\left({ }^{m n} G ; H\right),
$$

sempre que $P \in \mathcal{P}_{\mathcal{F}}\left({ }^{n} E ; F\right), Q \in \mathcal{P}\left({ }^{m} G ; E\right)$ e $t \in \mathcal{L}(F ; H)$. É claro que $t \circ P \circ Q$ é um polinômio $m n$-homogêneo de $G$ em $H$. Podemos escrever

$$
P=\sum_{j=1}^{k} P_{j} \otimes y_{j}
$$

onde $k \in \mathbb{N}, P_{j} \in \mathcal{P}\left({ }^{n} E\right)$ e $y_{j} \in F$ para todo $j=1, \ldots, k$. Então

$$
t \circ P \circ Q(x)=t\left(\sum_{j=1}^{k} P_{j} \otimes y_{j}(Q(x))\right)=\sum_{j=1}^{k} P_{j}(Q(x)) \cdot t\left(y_{j}\right)
$$

para todo $x \in G$. Disso segue que a imagem de $t \circ P \circ Q$ está contida no subespaço vetorial de $H$ gerado pelos vetores $t\left(y_{1}\right), \ldots, t\left(y_{k}\right)$; e portanto tem posto fnito, isto é, $t \circ P \circ Q \in \mathcal{P}_{\mathcal{F}}\left({ }^{m n} G ; H\right)$. 
Dado um hiper-ideal de polinômios $\mathcal{Q}$, queremos provar que $\mathcal{P}_{\mathcal{F}} \subseteq \mathcal{Q}$. Seja $Q \otimes y \in$ $\mathcal{P}_{\mathcal{F}}\left({ }^{n} E ; F\right)$, onde $Q \in \mathcal{P}\left({ }^{n} E\right)$ e $y \in F$. Podemos escrever

$$
Q \otimes y=(1 \otimes y) \circ Q
$$

onde $1 \otimes y: \mathbb{K} \longrightarrow F$ é dado por $1 \otimes y(\lambda)=\lambda y$. Como $1 \otimes y \in \mathcal{Q}(\mathbb{K} ; F)$ e $\mathcal{Q}$ é hiperideal de polinômios, da identidade destacada acima concluímos que $Q \otimes y \in \mathcal{Q}\left({ }^{n} E ; F\right)$. Como todo polinômio homogêneo de posto finito se escreve como uma soma finita de polinômios deste tipo, e como $\mathcal{Q}\left({ }^{n} E ; F\right)$ é subespaço vetorial de $\mathcal{P}\left({ }^{n} E ; F\right)$, segue que $\mathcal{P}_{\mathcal{F}}\left({ }^{n} E ; F\right) \subseteq \mathcal{Q}\left({ }^{n} E ; F\right)$. A desigualdade das normas segue da Proposição 3.1.5.

Com isso temos uma equivalência muito útil:

Corolário 3.2.3. Seja $\mathcal{Q}$ uma classe de polinômios homogêneos entre espaços de Banach que satisfaz a propriedade de hiper-ideal de polinômios. Para cada E, F espaços de Banach, são equivalentes:

(1) $\mathcal{Q}\left({ }^{n} E ; F\right)$ é um subespaço vetorial de $\mathcal{P}\left({ }^{n} E ; F\right)$ que contém os polinômios de tipo finito;

$\left(1^{\prime}\right) \mathcal{Q}\left({ }^{n} E ; F\right)$ é um subespaço vetorial de $\mathcal{P}\left({ }^{n} E ; F\right)$ que contém os polinômios de posto finito.

Exemplo 3.2.4. Como a classe dos polinômios de posto finito é um hiper-ideal, pela Proposição 3.1.4 decorre que a classe $\overline{\mathcal{P}_{\mathcal{F}}}$ dos polinômios aproximáveis na norma uniforme por polinômios de posto finito é um hiper-ideal fechado de polinômios.

Exemplo 3.2.5. Usando o polinômio $P$ do Exemplo 3.2.1, é possível mostrar, pelo mesmo argumento do Exemplo 2.2.4, que $P$ não é aproximável na norma uniforme por polinômios de tipo finito. Pelo Teorema 3.2.2 segue que a classe dos polinômios aproximáveis na norma uniforme por polinômios tipo finito não é um hiper-ideal de polinômios.

Para o próximo exemplo necessitamos introduzir uma classe de polinômios homogêneos bastante conhecida:

Definição 3.2.6. Um polinômio $n$-homogêneo $P \in \mathcal{P}\left({ }^{n} E ; F\right)$ é dito nuclear se podemos encontrar uma sequência limitada $\left(\varphi_{j}\right)_{j=1}^{\infty}$ de funcionais lineares em $E^{\prime}$, uma sequência $\left(\lambda_{j}\right)_{j=1}^{\infty} \in \ell_{1}$ e $\left(y_{j}\right)_{j=1}^{\infty}$ uma sequência limitada em $F$ tais que

$$
P(x)=\sum_{j=1}^{\infty} \lambda_{j} \cdot\left(\varphi_{j}(x)\right)^{n} \cdot y_{j}
$$


para qualquer $x \in E$. Nesse caso chamamos a expressão acima de representação nuclear de $P$ e escrevemos $P \in \mathcal{P}_{\mathcal{N}}\left({ }^{n} E ; F\right)$. Definimos ainda a função $\|\cdot\|_{\mathcal{P}_{\mathcal{N}}}: \mathcal{P}_{\mathcal{N}} \longrightarrow[0, \infty)$ por

$$
\|P\|_{\mathcal{P}_{\mathcal{N}}}=\inf \left\{\sum_{j=1}^{\infty}\left|\lambda_{j}\right| \cdot\left\|\varphi_{j}\right\|^{n} \cdot\left\|y_{j}\right\|\right\},
$$

onde o ínfimo é tomado sobre todas as representações nucleares de $P$.

Exemplo 3.2.7. Vejamos que a classe dos polinômios nucleares não forma um hiperideal de polinômios. Primeiramente notemos que, de forma análoga ao caso multilinear, é fácil verificar que a classe dos polinômios nucleares está contida na classe dos polinômios aproximáveis por polinômios de tipo finito. A demonstração de que $\mathcal{P}_{\mathcal{N}}$ é um ideal de polinômios é análoga à encontrada em [35, Proposition 2.2]. Em particular, $\|\cdot\| \leq\|\cdot\|_{\mathcal{P}_{\mathcal{N}}}$. Podemos agora concluir que a classe dos polinômios nucleares não é um hiper-ideal de polinômios. Para isso basta observar que a classe dos polinômios homogêneos aproximáveis por polinômios de tipo finito não contém os polinômios de posto finito. Então a classe dos polinômios nucleares também não pode contê-la, ou seja, não satisfaz a condição $\left(1^{\prime}\right)$ (condição necessária para a conclusão, de acordo com o Corolário 3.2.3)

Tendo em vista o exemplo acima, se faz necessário definir uma classe que desempenhe o papel de menor hiper-ideal de Banach de polinômios.

Definição 3.2.8. Sejam $s \in(0, \infty)$ e $r \in[1, \infty]$ tais que $1 \leq 1 / s+1 / r$. Um polinômio $n$-homogêneo $P \in \mathcal{P}\left({ }^{n} E ; F\right)$ é dito hiper- $(s ; r)$-nuclear se for possível encontrar escalares $\left(\lambda_{j}\right)_{j=1}^{\infty} \in \ell_{s}$, polinômios $\left(P_{j}\right)_{j=1}^{\infty} \in \ell_{r}^{w}\left(\mathcal{P}\left({ }^{n} E\right)\right)$ e vetores $\left(y_{j}\right)_{j=1}^{\infty} \in \ell_{\infty}(F)$ tais que

$$
P(x)=\sum_{j=1}^{\infty} \lambda_{j} P_{j} \otimes y_{j}(x)=\sum_{j=1}^{\infty} \lambda_{j} P_{j}(x) y_{j}
$$

para todo $x \in E$. Nesse caso escrevemos $P \in \mathcal{P}_{\mathcal{H N}_{(s, r)}}\left({ }^{n} E ; F\right)$ e dizemos que a expressão destacada acima é uma representação hiper- $(s ; r)$-nuclear de $P$. Definimos ainda a correspondência $\|\cdot\|_{\mathcal{P}_{\mathcal{H N}}(s, r)} \longrightarrow[0, \infty]$ por

$$
\|P\|_{\mathcal{P}_{\mathcal{H}}(s, r)}=\inf \left\{\left\|\left(\lambda_{j}\right)_{j=1}^{\infty}\right\|_{s} \cdot\left\|\left(P_{j}\right)_{j=1}^{\infty}\right\|_{r, w} \cdot\left\|\left(y_{j}\right)_{j=1}^{\infty}\right\|_{\infty}\right\},
$$

onde o ínfimo é tomado sobre todas as representações hiper- $(s ; r)$-nucleares de $P$.

Observação 3.2.9. Quando $s=1$ e $r=\infty$, obtemos a versão polinomial das aplicações multilineares hiper-nucleares. Nesse caso escrevemos simplesmente $\mathcal{P}_{\mathcal{H N}}$ e chamamos seus elementos de polinômios hiper-nucleares. 
Diversas constatações feitas no capítulo anterior, referentes a aplicações multilineares hiper- $(r, s)$-nucleares, podem ser reescritas, com as devidas adaptações, para polinômios. Várias delas, no presente texto, serviram apenas para auxiliar nas demonstrações dos resultados conclusivos sobre aplicações hiper- $(s, r)$-nucleares, por isso vamos omitir esses resultados preparatórios no caso de polinômios e enunciar apenas os resultados conclusivos.

Teorema 3.2.10. Dados $s \in(0, \infty)$ e $r \in[1, \infty]$, a classe $\mathcal{P}_{\mathcal{H N}_{(s, r)}}$ dos polinômios homogêneos hiper-(s;r)-nucleares, munida da função $\|\cdot\|_{\mathcal{P}_{\mathcal{H N}_{(s, r)}}}$, é um hiper-ideal p-Banach de polinômios, onde $1 / p=1 / s+1 / r$.

Demonstração. O resultado segue utilizando o Critério da Série para hiper-ideais de polinômios (Teorema 3.1.7) e seguindo os mesmos passos da demonstração do Teorema 2.2.9, com as devidas adaptações.

Teorema 3.2.11. A classe $\mathcal{P}_{\mathcal{H N}}$ dos polinômios hiper-nucleares, munida da função $\| \cdot$ $\|_{\mathcal{P}_{\mathcal{H N}}}$, forma o menor hiper-ideal de Banach de polinômios. Isso significa que $\left(\mathcal{P}_{\mathcal{H N}}, \| \cdot\right.$ $\left.\|_{\mathcal{P}_{\mathcal{H N}}}\right)$ é um hiper-ideal de Banach de polinômios e se $\left(\mathcal{Q},\|\cdot\|_{\mathcal{Q}}\right)$ também o é, então $\mathcal{P}_{\mathcal{H N}} \subseteq \mathcal{Q}$ e $\|P\|_{\mathcal{Q}} \leq C_{1, n}\|P\|_{\mathcal{P}_{\mathcal{H N}}}$ para todos $n \in \mathbb{N}$ e $P \in \mathcal{P}_{\mathcal{H N}}\left({ }^{n} E ; F\right)$.

Demonstração. A demonstração segue a mesma linha da demonstração do Teorema 2.2.12. Vale ressaltar que, seguindo os passos daquela demonstração, trabalharemos com uma sequência de polinômios $n$-homogêneos $\left(Q_{k}\right)_{k=1}^{\infty}$ que satisfaz uma estimativa análoga à estimativa que aparece em (2.5). Neste caso tomamos $\frac{\varepsilon}{C_{1, n}}$ ao invés de $\varepsilon$. Dessa forma, se $Q_{k}:=\sum_{j=1}^{k} \lambda_{j} P_{j} \otimes y_{j}$, obtemos em seguida que

$$
\left\|Q_{k}-Q_{i}\right\| \leq C_{1, n} \cdot \sum_{j=1}^{\infty}\left|\lambda_{j}\right| \cdot\left\|P_{j}\right\| \cdot\left\|y_{j}\right\|<\varepsilon
$$

nos permitindo concluir, também neste caso, que $\left(Q_{k}\right)_{k=1}^{\infty}$ é uma sequência de Cauchy. Continuando os mesmos passos da demonstração supracitada chegamos aos resultados. 


\subsection{Hiper-ideais de polinômios gerados por hiper-ideais de aplicações multilineares}

Além de ensinar como obter hiper-ideais de polinômios homogêneos a partir de hiperideais de aplicações multilineares, os procedimentos estabelecidos nesta seção serão muito úteis no sentido de simplificar as demonstrações dos resultados da seção seguinte. Por $\mathcal{P}$ denotamos a classe de todos os polinômios homogêneos contínuos entre espaços de Banach.

Definição 3.3.1. Seja $\mathcal{G}$ uma subclasse da classe de aplicações multilineares contínuas entre espaços de Banach. Definimos

$$
\mathcal{P}^{\mathcal{G}}:=\{P \in \mathcal{P}: \text { existe } A \in \mathcal{G} \text { tal que } P=\widehat{A}\} .
$$

Para cada função $\|\cdot\|_{\mathcal{G}}: \mathcal{G} \longrightarrow \mathbb{R}$, definimos a função correspondente $\|\cdot\|_{\mathcal{P}^{\mathcal{G}}}: \mathcal{P}^{\mathcal{G}} \longrightarrow \mathbb{R}$ por

$$
\|P\|_{\mathcal{P}^{\mathcal{G}}}=\inf \left\{\|A\|_{\mathcal{G}}: A \in \mathcal{G} \text { e } P=\widehat{A}\right\}
$$

O próximo resultado nos diz que essa é uma fonte de hiper-ideais de polinômios gerados a partir de hiper-ideais de aplicações multilineares.

Teorema 3.3.2. (i) Se $\mathcal{H}$ é um hiper-ideal, então $\mathcal{P}^{\mathcal{H}}$ é um hiper-ideal de polinômios.

(ii) Se $\left(\mathcal{H},\|\cdot\|_{\mathcal{H}}\right)$ é um hiper-ideal p-normado (p-Banach), então $\left(\mathcal{P}^{\mathcal{H}},\|\cdot\|_{\mathcal{P}_{\mathcal{H}}}\right)$ é um hiper-ideal p-normado (p-Banach) de polinômios.

Demonstração. (i) Verifiquemos as condições da Definição 3.1:

(1) Claramente $\mathcal{P}^{\mathcal{H}}\left({ }^{n} E ; F\right)$ é um subespaço vetorial de $\mathcal{P}\left({ }^{n} E ; F\right)$, para todo $n \in \mathbb{N}$ e quaisquer espaços de Banach $E$ e $F$. Agora seja $P \in \mathcal{P}\left({ }^{n} E ; F\right)$ de tipo finito, digamos,

$$
P=\sum_{j=1}^{k} \varphi_{j}^{n} \otimes y_{j}
$$

onde $\varphi_{j} \in E^{\prime}$ e $y_{j} \in F, j=1, \ldots, k$. Então

$$
\check{P}=\sum_{j=1}^{k} \varphi_{j} \otimes \cdots \otimes \varphi_{j} \otimes y_{j} \in \mathcal{L}_{f}\left({ }^{n} E ; F\right) \subseteq \mathcal{H}\left({ }^{n} E ; F\right),
$$

onde cada $\varphi_{j}$ aparece $n$ vezes e a última inclusão decorre do fato de $\mathcal{H}$ ser um hiperideal. Como $P=(\check{P})^{\wedge}$ segue que $P \in \mathcal{P}^{\mathcal{H}}\left({ }^{n} E ; F\right)$, ou seja, $\mathcal{P}^{\mathcal{H}}$ contém os polinômios homogêneos de tipo finito. 
(2) Sejam $t \in \mathcal{L}(F ; H), Q \in \mathcal{P}^{\mathcal{H}}\left({ }^{n} E ; F\right)$ e $R \in \mathcal{P}\left({ }^{m} G ; E\right)$. Como $Q \in \mathcal{P}^{\mathcal{H}}\left({ }^{n} E ; F\right)$, existe $A \in \mathcal{H}\left({ }^{n} E ; F\right)$ tal que $\widehat{A}=Q$. Seja ainda $B \in \mathcal{L}\left({ }^{m} G ; E\right)$ tal que $\widehat{B}=R$. Então,

$$
\begin{aligned}
(t \circ A \circ(B, \ldots, B))^{\wedge}(x) & =t(A(B(x, \ldots, x), \ldots, B(x, \ldots, x)))=t(A(R(x), \ldots, R(x))) \\
& =t(Q(R(x)))=t \circ Q \circ R(x)
\end{aligned}
$$

para todo $x \in G$. Sendo $\mathcal{H}$ hiper-ideal, $\left.t \circ A \circ(B, \ldots, B) \in \mathcal{H}^{(m n} G ; H\right)$. Portanto $t \circ Q \circ R \in \mathcal{P}^{\mathcal{H}}\left({ }^{m n} G ; H\right)$. Logo $\mathcal{P}^{\mathcal{H}}$ é hiper-ideal de polinômios.

(ii) Verifiquemos as propriedades da correspondência $\|\cdot\|_{\mathcal{P}_{\mathcal{H}}}$ :

(a) Devemos mostrar que $\left\|\widehat{I_{n}}\right\|_{\mathcal{P}_{\mathcal{H}}}=1$ para todo $n \in \mathbb{N}$. Dado $n \in \mathbb{N}$, pela definição de $\|\cdot\|_{\mathcal{P} \mathcal{H}}$ temos

$$
\left\|\widehat{I}_{n}\right\|_{\mathcal{P}^{\mathcal{H}}} \leq\left\|I_{n}\right\|_{\mathcal{H}}=1
$$

Supondo $\left\|\widehat{I_{n}}\right\|_{\mathcal{P}_{\mathcal{H}}}<1$, existiria uma forma $n$-linear $A \in \mathcal{H}\left({ }^{n} \mathbb{K}\right)$ tal que $A(\lambda)=\lambda^{n}$ para todo $\lambda \in \mathbb{K}$ e $\|A\|_{\mathcal{H}}<1$. Neste caso obteríamos

$$
1=|A(1, \ldots, 1)| \leq\|A\| \leq\|A\|_{\mathcal{H}}<1
$$

contradição esta que prova que $\left\|\widehat{I_{n}}\right\|_{\mathcal{P}_{\mathcal{H}}}=1$.

(b) Seja $P \in \mathcal{P}^{\mathcal{H}}\left({ }^{n} E ; F\right)$. Supondo $\|P\|_{\mathcal{P}_{\mathcal{H}}}=0$, para todo $\varepsilon>0$ existe $A \in \mathcal{H}\left({ }^{n} E ; F\right)$ tal que $\|A\|_{\mathcal{H}}<\varepsilon$. Assim,

$$
\|P\|=\|\widehat{A}\| \leq\|A\| \leq\|A\|_{\mathcal{H}}<\varepsilon,
$$

o que implica $P=0$. Para todo $\lambda \in \mathbb{K}$, mantendo a notação acima, temos

$$
\|\lambda P\|_{\mathcal{P}_{\mathcal{H}}} \leq\|\lambda A\|_{\mathcal{H}}=|\lambda| \cdot\|A\|_{\mathcal{H}}
$$

Como $A$ é arbitrária, obtemos $\|\lambda P\|_{\mathcal{P}_{\mathcal{H}}} \leq|\lambda| \cdot\|P\|_{\mathcal{P H}_{\mathcal{H}}}$. Disso segue que

$$
\|P\|_{\mathcal{P}^{\mathcal{H}}}=\left\|\frac{1}{\lambda}(\lambda P)\right\|_{\mathcal{P}_{\mathcal{H}}} \leq \frac{1}{|\lambda|}\|\lambda P\|_{\mathcal{P}_{\mathcal{H}}} \Longrightarrow|\lambda| \cdot\|P\|_{\mathcal{P}^{\mathcal{H}}} \leq\|\lambda P\|_{\mathcal{P}^{\mathcal{H}}},
$$

para $\lambda \neq 0$. Portanto $\|\lambda P\|_{\mathcal{P}_{\mathcal{H}}}=|\lambda| \cdot\|P\|_{\mathcal{P}_{\mathcal{H}}}$ para todo $\lambda \in \mathbb{K}$ com a ressalva de que para $\lambda=0$ a igualdade é obvia.

Se também tivermos $Q \in \mathcal{P}^{\mathcal{H}}\left({ }^{n} E ; F\right)$, tomando $B \in \mathcal{H}\left({ }^{n} E ; F\right)$ tal que $Q=\widehat{B}$, de

$$
\widehat{P+Q}=\widehat{P}+\widehat{Q}=A+B
$$

segue que

$$
\|P+Q\|_{\mathcal{P}^{\mathcal{H}}}^{p} \leq\|A+B\|_{\mathcal{H}}^{p} \leq\|A\|_{\mathcal{H}}^{p}+\|B\|_{\mathcal{H}}^{p} .
$$


Tomando o ínfimo sobre tais aplicações $A$ e $B$, obtemos a desigualdade $p$-triangular

$$
\|P+Q\|_{\mathcal{P H}}^{p} \leq\|P\|_{\mathcal{P}_{\mathcal{H}}}^{p}+\|Q\|_{\mathcal{P} \mathcal{H}}^{p}
$$

Portanto $\|\cdot\|_{\mathcal{P H}}$ é uma $p$-norma.

(c) Seguindo com a notação do item (2) desta demonstração, da desigualdade de hiperideal de $\mathcal{H}$ temos

$$
\begin{aligned}
\|t \circ Q \circ R\|_{\mathcal{P}_{\mathcal{H}}} & \leq\|t \circ A \circ(B, \ldots, B)\|_{\mathcal{H}} \leq\|t\| \cdot\|A\|_{\mathcal{H}} \cdot\|B\|^{n} \\
& \leq\|t\| \cdot\|A\|_{\mathcal{H}} \cdot\left(\frac{m^{m}}{m !}\|\widehat{B}\|\right)^{n}=C_{n, m}\|t\| \cdot\|A\|_{\mathcal{H}} \cdot\|R\|^{n} .
\end{aligned}
$$

Disso segue que $\|t \circ Q \circ R\|_{\mathcal{P}_{\mathcal{H}}} \leq C_{n, m}\|t\| \cdot\|Q\|_{\mathcal{H}} \cdot\|R\|^{n}$.

Resta mostrar que se $\left(\mathcal{H},\|\cdot\|_{\mathcal{H}}\right)$ é completo, então $\left(\mathcal{P}^{\mathcal{H}},\|\cdot\|_{\mathcal{P}^{\mathcal{H}}}\right)$ também é completo. Para isso seja $\left(P_{j}\right)_{j=1}^{\infty}$ em $\mathcal{P}^{\mathcal{H}}\left({ }^{n} E ; F\right)$ tal que $\sum_{j=1}^{\infty}\left\|P_{j}\right\|_{\mathcal{P}_{\mathcal{H}}}^{p}<\infty$. Devemos mostrar que a série $\sum_{j=1}^{\infty} P_{j}$ converge em $\mathcal{P}^{\mathcal{H}}\left({ }^{n} E ; F\right)$. Para isso seja $\varepsilon>0$. Sabemos que, para cada $j \in \mathbb{N}$, existe uma aplicação $n$-linear $A_{j} \in \mathcal{H}\left({ }^{n} E ; F\right)$ tal que

$$
\widehat{A}_{j}=P_{j} \text { e }\left\|A_{j}\right\|_{\mathcal{H}}<(1+\varepsilon)\left\|P_{j}\right\|_{\mathcal{P}^{\mathcal{H}}}
$$

Como

$$
\sum_{j=1}^{\infty}\left\|A_{j}\right\|_{\mathcal{H}}^{p}<(1+\varepsilon)^{p} \cdot \sum_{j=1}^{\infty}\left\|P_{j}\right\|_{\mathcal{P}^{\mathcal{H}}}^{p}<\infty
$$

temos pela completude de $\mathcal{H}$ que a série $\sum_{j=1}^{\infty} A_{j}$ converge em $\mathcal{H}\left({ }^{n} E ; F\right)$, digamos $A=$ $\sum_{j=1}^{\infty} A_{j}$ com convergência em $\mathcal{H}\left({ }^{n} E ; F\right)$. Podemos então tomar $P:=\widehat{A} \in \mathcal{P}\left({ }^{n} E ; F\right)$. Pela definição de $\mathcal{P}^{\mathcal{H}}$ segue que $P \in \mathcal{P}^{\mathcal{H}}\left({ }^{n} E ; F\right)$. Finalmente,

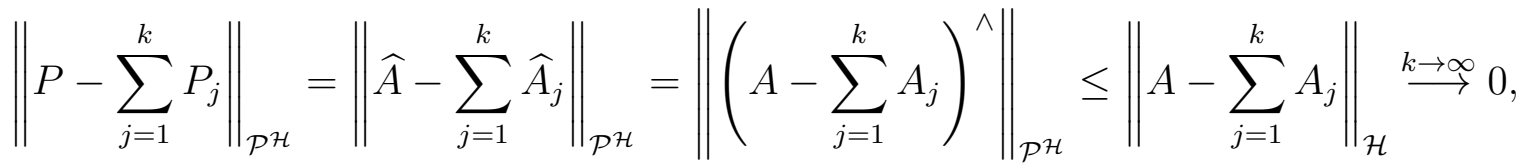

provando que $P=\sum_{j=1}^{\infty} P_{j}$ em $\mathcal{P}^{\mathcal{H}}\left({ }^{n} E ; F\right)$

Uma segunda tentativa de gerar hiper-ideais de polinômios a partir de um hiper-ideal é considerar a seguinte definição: 
Definição 3.3.3. Seja $\mathcal{G}$ uma subclasse da classe das aplicações multilineares contínuas entre espaços de Banach. Definimos

$$
\mathcal{P}_{\mathcal{G}}:=\{P \in \mathcal{P}: \check{P} \in \mathcal{G}\}
$$

Para cada função $\|\cdot\|_{\mathcal{G}}: \mathcal{G} \longrightarrow[0, \infty)$, definimos a função correspondente $\|\cdot\|_{\mathcal{P}_{\mathcal{G}}}: \mathcal{P}_{\mathcal{G}} \longrightarrow$ $[0, \infty)$ por

$$
\|P\|_{\mathcal{P}_{\mathcal{G}}}=\|\check{P}\|_{\mathcal{G}}
$$

Observe que, para toda classe $\mathcal{G}$ de aplicações multilineares munida de uma função $\|\cdot\|_{\mathcal{G}}$, tem-se

$$
\mathcal{P}_{\mathcal{G}} \subseteq \mathcal{P}^{\mathcal{G}} \text { e }\|\cdot\|_{\mathcal{P}^{\mathcal{G}}} \leq\|\cdot\|_{\mathcal{P}_{\mathcal{G}}}
$$

Se $\mathcal{M}$ é um multi-ideal, é fácil verificar que $\mathcal{P}_{\mathcal{M}}$ é um ideal de polinômios. Entretanto, para hiper-ideais não há esperança desse procedimento funcionar em geral. De fato, o problema se torna evidente ao pensarmos em composições à esquerda por polinômios $n$ homogêneos com $n>1$. Quando estamos trabalhando apenas com a definição de ideais de polinômios, para que $P \circ u$ esteja em $\mathcal{P}_{\mathcal{M}}$, onde $u$ é um operador linear, basta que $P \in \mathcal{P}_{\mathcal{M}}$, visto que

$$
(P \circ u)^{\vee}=\check{P} \circ(u, \ldots, u),
$$

e segue da definição de multi-ideais que $\check{P} \circ(u, \ldots, u) \in \mathcal{M}$. Entretanto, quando trabalhamos com hiper-ideais de polinômios e compomos à esquerda por um polinômio não-linear, as coisas certamente se complicam da seguinte forma: suponha que $\mathcal{H}$ seja um hiper-ideal de aplicações multilineares e tome $P \in \mathcal{P}_{\mathcal{H}}$. Sabemos que $\check{P} \in \mathcal{H}$. Para que $\mathcal{P}_{\mathcal{H}}$ seja um hiper-ideal de polinômios devemos ter $P \circ Q$ em $\mathcal{P}_{\mathcal{H}}$ para todo polinômio homogêneo $Q$ com domínio e contra-domínios compatíveis para a composição. Queremos então que $(P \circ Q)^{\vee}$ pertença a $\mathcal{H}$. Como $\mathcal{H}$ é hiper-ideal, sabemos apenas que $\check{P} \circ(\check{Q}, \ldots, \check{Q})$ pertence a $\mathcal{H}$, e em geral não é verdade que

$$
(P \circ Q)^{\vee}=\check{P} \circ(\check{Q}, \ldots, \check{Q}),
$$

pois a aplicação $\check{P} \circ(\check{Q}, \ldots, \check{Q})$ não é nem simétrica em geral.

Para contornar esta dificuldade devemos introduzir o conceito de simetrização de uma aplicação multilinear.

Definição 3.3.4. Dadas uma aplicação $A \in \mathcal{L}\left({ }^{n} E ; F\right)$ e uma permutação $\sigma \in S_{n}$, defini$\operatorname{mos} A_{\sigma} \in \mathcal{L}\left({ }^{n} E ; F\right)$ por

$$
A_{\sigma}\left(x_{1}, \ldots, x_{n}\right)=A\left(x_{\sigma(1)}, \ldots, x_{\sigma(n)}\right)
$$


Definimos agora $A_{s} \in \mathcal{L}\left({ }^{n} E ; F\right)$ por

$$
A_{s}\left(x_{1}, \ldots, x_{n}\right)=\frac{1}{n !} \cdot \sum_{\sigma \in S_{n}} A\left(x_{\sigma(1)}, \ldots, x_{\sigma(n)}\right)=\frac{1}{n !} \cdot \sum_{\sigma \in S_{n}} A_{\sigma}\left(x_{1}, \ldots, x_{n}\right),
$$

aplicação esta que é chamada de simetrização de $A$.

Dizemos que uma subclasse $\mathcal{G}$ da classe das aplicações multilineares contínuas entre espaços de Banach é simétrica se, para quaisquer espaços de Banach $E, F$ e $n \in \mathbb{N}$, $A_{s} \in \mathcal{G}\left({ }^{n} E ; F\right)$ sempre que $A \in \mathcal{G}\left({ }^{n} E ; F\right)$.

Dada uma função $\|\cdot\|_{\mathcal{G}}: \mathcal{G} \longrightarrow[0, \infty)$, dizemos que $\left(\mathcal{G},\|\cdot\|_{\mathcal{G}}\right)$ é fortemente simétrica se

$$
A_{\sigma} \in \mathcal{G} \text { e }\left\|A_{\sigma}\right\|_{\mathcal{G}}=\|A\|_{\mathcal{G}}
$$

para quaisquer $A \in \mathcal{G}\left({ }^{n} E ; F\right)$ e $\sigma \in S_{n}$.

Lema 3.3.5. Uma subclasse $\mathcal{G}$ da classe das aplicações multilineares contínuas entre espaços de Banach é simétrica se, e somente se, $\mathcal{P}^{\mathcal{G}}=\mathcal{P}_{\mathcal{G}}$.

Demonstração. Suponha que $\mathcal{G}$ seja simétrica. Dado um polinômio $P \in \mathcal{P}_{\mathcal{G}}$, por (3.1) segue que $P \in \mathcal{P}^{\mathcal{G}}$. Para a inclusão inversa, dado um polinômio $P \in \mathcal{P}^{\mathcal{G}}$, podemos tomar $A \in \mathcal{G}$ tal que $P=\widehat{A}$. Como a classe $\mathcal{G}$ é simétrica, segue que $A_{s} \in \mathcal{G}$. De

$$
\check{P}=(\widehat{A})^{\vee}=A_{s}
$$

concluímos que $\check{P} \in \mathcal{G}$, isto é $P \in \mathcal{P}_{\mathcal{G}}$. Assim $\mathcal{P}^{\mathcal{G}}=\mathcal{P}_{\mathcal{G}}$.

Reciprocamente, suponhamos que $\mathcal{P}^{\mathcal{G}}=\mathcal{P}_{\mathcal{G}}$. Dada $A \in \mathcal{H}$, por hipótese $P:=\widehat{A} \in$ $\mathcal{P}^{\mathcal{H}}$, e por hipótese $\check{P} \in \mathcal{H}$. Assim como antes, $A_{s}=(\widehat{A})^{\vee}=\check{P} \in \mathcal{H}$. Portanto $\mathcal{H}$ é simétrica.

Proposição 3.3.6. (i) Se $\mathcal{H}$ é um hiper-ideal simétrico de aplicações multilineares, então $\mathcal{P}^{\mathcal{H}}=\mathcal{P}_{\mathcal{H}}$ e, em particular, $\mathcal{P}_{\mathcal{H}}$ é um hiper-ideal de polinômios.

(ii) Se $0<p \leq 1 e\left(\mathcal{H},\|\cdot\|_{\mathcal{H}}\right)$ é um hiper-ideal p-normado fortemente simétrico de aplicações multilineares, então

$$
\|P\|_{\mathcal{P}_{\mathcal{H}}} \leq\|P\|_{\mathcal{P}_{\mathcal{H}}} \leq(n !)^{\frac{1}{p}-1}\|P\|_{\mathcal{P}_{\mathcal{H}}}
$$

para todos $n \in \mathbb{N}$ e $P \in \mathcal{P}_{\mathcal{H}}\left({ }^{n} E ; F\right)$.

(iii) $\operatorname{Se}\left(\mathcal{H},\|\cdot\|_{\mathcal{H}}\right)$ é um hiper-ideal normado (Banach, respectivamente) fortemente simétrico de aplicações multilineares, então $\left(\mathcal{P}_{\mathcal{H}},\|\cdot\|_{\mathcal{P}_{\mathcal{H}}}\right)$ é hiper-ideal normado (Banach, respectivamente) de polinômios. 
Demonstração. (i) Como $\mathcal{H}$ é simétrico, do Lema 3.3.5 segue que $\mathcal{P}^{\mathcal{H}}=\mathcal{P}_{\mathcal{H}}$, e sabemos que $\mathcal{P}^{\mathcal{H}}$ é hiper-ideal de polinômios pelo Teorema 3.3.2.

(ii) Basta provar a segunda desigualdade. Seja $P \in \mathcal{P}_{\mathcal{H}}\left({ }^{n} E ; F\right)$. Para toda aplicação $n$ linear $A \in \mathcal{H}\left({ }^{n} E ; F\right)$ tal que $\widehat{A}=P$, tem-se $\check{P}=A_{s}$. Disso, da desigualdade $p$-triangular e do fato de $\left(\mathcal{H},\|\cdot\|_{\mathcal{H}}\right)$ ser fortemente simétrico, segue que

$$
\begin{aligned}
\|P\|_{\mathcal{P}_{\mathcal{H}}} & =\left(\|P\|_{\mathcal{P}_{\mathcal{H}}}^{p}\right)^{1 / p}=\left(\|\check{P}\|_{\mathcal{H}}^{p}\right)^{1 / p}=\left(\left\|A_{s}\right\|_{\mathcal{H}}^{p}\right)^{1 / p}=\left(\left\|\frac{1}{n !} \cdot \sum_{\sigma \in S_{n}} A_{\sigma}\right\|_{\mathcal{H}}^{p}\right)^{1 / p} \\
& \leq \frac{1}{n !} \cdot\left(\sum_{\sigma \in S_{n}}\left\|A_{\sigma}\right\|_{\mathcal{H}}^{p}\right)^{1 / p}=\frac{1}{n !} \cdot\left(\sum_{\sigma \in S_{n}}\|A\|_{\mathcal{H}}^{p}\right)^{1 / p}=\frac{(n !)^{1 / p}}{n !}\|A\|_{\mathcal{H}} .
\end{aligned}
$$

(iii) De (i) temos $\mathcal{P}^{\mathcal{H}}=\mathcal{P}_{\mathcal{H}}$, e tomando $p=1 \mathrm{em}$ (ii) obtemos $\|\cdot\|_{\mathcal{P}_{\mathcal{H}}}=\|\cdot\|_{\mathcal{P}_{\mathcal{H}}}$. O resultado segue do Teorema 3.3.2(ii).

\subsection{Outros métodos para gerar hiper-ideais de po- linômios}

Na seção anterior, obtivemos duas fontes de exemplos de hiper-ideais de polinômios $p$-normados obtidos através de hiper-ideais de aplicações multilineares $p$-normadas, com as ressalvas que um desses métodos exige que o hiper-ideal seja simérico e que mesmo coincidindo como conjuntos, as p-normas obtidas só coincidem no caso normado, isto é, $p=1$. Mas a primeira restrição não se aplica aos exemplos do capítulo anterior, visto que é possível verificar que todos os hiper-ideais lá apresentados são simétricos. O que faremos nas próximas subseções é adaptar os métodos descritos no Capítulo 2 de modo a obter métodos para gerar hiper-ideais de polinômios e comparar com os hiper-ideais gerados pelos procedimentos $\left(\mathcal{P}_{\mathcal{H}},\|\cdot\|_{\mathcal{P}_{\mathcal{H}}}\right)$ e $\left(\mathcal{P}^{\mathcal{H}},\|\cdot\|_{\mathcal{P}_{\mathcal{H}}}\right)$ da seção anterior, incluindo suas normas.

\subsubsection{Método da fatoração}

Dentre as várias tentativas descritas na Seção 2.3, adaptamos para polinômios homogêneos aquela que consideramos mais bem sucedida, isto é, o procedimento da Definição 2.3.8. 
Definição 3.4.1. Sejam $\left(\mathcal{Q},\|\cdot\|_{\mathcal{Q}}\right)$ um hiper-ideal de polinômios $p$-normado e $P \in$ $\mathcal{P}\left({ }^{n} E ; F\right)$. Se existirem $k, m_{1}, \ldots, m_{k}, r_{1}, \ldots, r_{k} \in \mathbb{N}$, espaços de Banach $G_{j}$ e polinômios homogêneos $Q_{j} \in \mathcal{P}\left({ }^{m_{j}} G_{j} ; F\right)$ e $R_{j} \in \mathcal{Q}\left({ }^{r_{j}} E ; G_{j}\right)$, com $m_{j} \cdot r_{j}=n$, para todo $j=1, \ldots, k$, tais que

$$
P=\sum_{j=1}^{k} Q_{j} \circ R_{j}
$$

então escrevemos $P \in \mathcal{P}(\mathcal{Q})\left({ }^{n} E ; F\right)$. Chamamos (3.2) de fatoração de $P$ por $\mathcal{Q}$. Definimos ainda $\|\cdot\|_{\mathcal{P}(\mathcal{Q})}: \mathcal{P}(\mathcal{Q}) \longrightarrow[0, \infty)$, por

$$
\|P\|_{\mathcal{P}(\mathcal{Q})}=\inf \left\{\sum_{j=1}^{k}\left\|Q_{j}\right\| \cdot\left\|R_{j}\right\|_{\mathcal{Q}}^{r_{j}}\right\}
$$

onde o ínfimo é tomado sobre todas as fatorações de $P$ por $\mathcal{Q}$.

Proposição 3.4.2. Sejam $0<p \leq 1 e\left(\mathcal{Q},\|\cdot\|_{\mathcal{Q}}\right)$ um hiper-ideal p-normado de polinômios. Então $\left(\mathcal{P}(\mathcal{Q}),\|\cdot\|_{\mathcal{P}(\mathcal{Q})}\right)$ é um hiper-ideal p-normado de polinômios que contém $\mathcal{Q}$.

Demonstração. A demonstração deste resultado é análoga à do Teorema 2.3.9.

Dado um hiper-ideal $\mathcal{H}$, sob certas condições podemos considerar, por um lado, o hiper-ideal $\mathcal{L}(\mathcal{H})$, e portanto o hiper-ideal de polinômios $\mathcal{P}_{\mathcal{L}(\mathcal{H})} ;$ e, por outro lado, $\mathcal{P}_{\mathcal{H}}$ e, consequentemente, $\mathcal{P}\left(\mathcal{P}_{\mathcal{H}}\right)$. Em relação a essas classes, temos:

Teorema 3.4.3. Seja $\left(\mathcal{H},\|\cdot\|_{\mathcal{H}}\right)$ um hiper-ideal normado tal que $\mathcal{L}(\mathcal{H})$ também é um hiper-ideal. Então:

(i) A inclusão $\mathcal{P}\left(\mathcal{P}^{\mathcal{H}}\right) \subseteq \mathcal{P}^{\mathcal{L}(\mathcal{H})}$ é sempre verdadeira. Além disso, para todo polinômio n-homogêneo $P$ em $\mathcal{P}\left(\mathcal{P}^{\mathcal{H}}\right)$, vale

$$
\|P\|_{\mathcal{P} \mathcal{L}(\mathcal{H})} \leq e^{n}\|P\|_{\mathcal{P}\left(\mathcal{P}^{\mathcal{H}}\right)}
$$

(ii) Se $\mathcal{H}$ e $\mathcal{L}(\mathcal{H})$ são simétricos, então $\mathcal{P}\left(\mathcal{P}_{\mathcal{H}}\right) \subseteq \mathcal{P}_{\mathcal{L}(\mathcal{H})}$. Além disso, para todo polinômio n-homogêneo $P$ em $\mathcal{P}\left(\mathcal{P}_{\mathcal{H}}\right)$, vale

$$
\|P\|_{\mathcal{P}_{\mathcal{L}(\mathcal{H})}} \leq e^{n}\|P\|_{\mathcal{P}\left(\mathcal{P}_{\mathcal{H}}\right)}
$$

Demonstração. (i) Dado $P \in \mathcal{P}\left(\mathcal{P}^{\mathcal{H}}\right)\left({ }^{n} E ; F\right)$, tome polinômios $Q_{j} \in \mathcal{P}\left({ }^{m_{j}} G_{j} ; F\right)$ e $R_{j} \in$ $\mathcal{P}^{\mathcal{H}}\left({ }^{r_{j}} E ; G_{j}\right), j=1, \ldots, k$, de acordo com a Definição 3.4.1. Então

$$
P=\sum_{j=1}^{k} Q_{j} \circ R_{j}
$$


Cada $R_{j} \in \mathcal{P}^{\mathcal{H}}\left({ }^{r_{j}} E ; G_{j}\right)$, logo existe $A_{j} \in \mathcal{H}\left({ }^{r_{j}} E ; G_{j}\right)$ de modo que $\widehat{A}_{j}=R_{j}$. Dessa forma

$$
\check{Q}_{j} \circ\left(\widehat{A}_{j}, \ldots, \widehat{A}_{j}\right) \in \mathcal{L}(\mathcal{H})\left({ }^{n} E ; F\right)
$$

para todo $j=1, \ldots, k$. Definindo $A:=\sum_{j=1}^{k} \check{Q}_{j} \circ\left(\widehat{A}_{j}, \ldots, \widehat{A}_{j}\right)$, temos $A \in \mathcal{L}(\mathcal{H})\left({ }^{n} E ; F\right)$ e

$$
\begin{aligned}
\widehat{A}(x) & =\sum_{j=1}^{k}\left(\check{Q}_{j} \circ\left(A_{j}, \ldots, A_{j}\right)\right)(x, \ldots, x)=\sum_{j=1}^{k}\left(\check{Q}_{j} \circ\left(A_{j}(x, \ldots, x), \ldots, A_{j}(x, \ldots, x)\right)\right) \\
& =\sum_{j=1}^{k}\left(\check{Q}_{j} \circ\left(R_{j}(x), \ldots, R_{j}(x)\right)\right)=\sum_{j=1}^{k} Q_{j} \circ R_{j}(x)=P(x),
\end{aligned}
$$

para todo $x \in E$. Portanto $P \in \mathcal{P}^{\mathcal{L}(\mathcal{H})}\left({ }^{n} E ; F\right)$.

Considerando a representação (3.3) de $P$, pela definição de $\|\cdot\|_{\mathcal{P} \mathcal{L}(\mathcal{H})}$, temos

$$
\begin{aligned}
\|P\|_{\mathcal{P} \mathcal{L}(\mathcal{H})} & \leq\|A\|_{\mathcal{L}(\mathcal{H})} \leq \sum_{j=1}^{k}\left\|\check{Q}_{j}\right\| \cdot\left\|A_{j}\right\|_{\mathcal{H}}^{r_{j}} \leq \sum_{j=1}^{k} \frac{m_{j}^{m_{j}}}{m_{j} !}\left\|Q_{j}\right\| \cdot\left\|A_{j}\right\|_{\mathcal{H}}^{r_{j}} \\
& \leq \sum_{j=1}^{k} e^{m_{j}}\left\|Q_{j}\right\| \cdot\left\|A_{j}\right\|_{\mathcal{H}}^{r_{j}} \leq e^{n} \cdot \sum_{j=1}^{k}\left\|Q_{j}\right\| \cdot\left\|A_{j}\right\|_{\mathcal{H}}^{r_{j}} .
\end{aligned}
$$

Como cada $A_{j}$ foi tomada arbitrariamente de modo que $\widehat{A}_{j}=R_{j}$, da desigualdade acima segue que

$$
\|P\|_{\mathcal{P} \mathcal{L}(\mathcal{H})} \leq e^{n} \cdot \sum_{j=1}^{k}\left\|Q_{j}\right\| \cdot\left\|R_{j}\right\|_{\mathcal{P} \mathcal{H}}^{r_{j}} .
$$

Agora, obervando que a representação (3.3) de $P$ também é arbitrária, concluímos $\|P\|_{\mathcal{P} \mathcal{L}(\mathcal{H})} \leq$ $e^{n}\|P\|_{\mathcal{P}(\mathcal{P} \mathcal{H})}$.

(ii) Suponhamos agora $\mathcal{H}$ um hiper-ideal simétrico, $\mathcal{P}_{\mathcal{H}}$ é um hiper-ideal de polinômios pela Proposição 3.3.6, e então faz sentido considerar $\mathcal{P}\left(\mathcal{P}_{\mathcal{H}}\right)$. Dado $P \in \mathcal{P}\left(\mathcal{P}_{\mathcal{H}}\right)\left({ }^{n} E ; F\right)$, tome polinômios $Q_{j} \in \mathcal{P}\left({ }^{m_{j}} G_{j} ; F\right)$ e $R_{j} \in \mathcal{P}_{\mathcal{H}}\left({ }^{r_{j}} E ; G_{j}\right), j=1, \ldots, k$, de acordo com a Definição 3.4.1. Então

$$
P=\sum_{j=1}^{k} Q_{j} \circ R_{j} .
$$

Cada $R_{j} \in \mathcal{P}_{\mathcal{H}}\left({ }^{r_{j}} E ; G_{j}\right), \operatorname{logo} \check{R}_{j} \in \mathcal{H}\left({ }^{r_{j}} E ; G_{j}\right)$. Dessa forma

$$
\check{Q}_{j} \circ\left(\check{R}_{j}, \ldots, \check{R}_{j}\right) \in \mathcal{L}(\mathcal{H})\left({ }^{n} E ; F\right),
$$

para todo $j=1, \ldots, k$. Da simetria de $\mathcal{L}(\mathcal{H})$ segue que $\left(\check{Q}_{j} \circ\left(\check{R}_{j}, \ldots, \check{R}_{j}\right)\right)_{s} \in \mathcal{L}(\mathcal{H})\left({ }^{n} E ; F\right)$, e portanto

$$
\check{P}=\left(\check{Q}_{j} \circ\left(\check{R}_{j}, \ldots, \check{R}_{j}\right)\right)_{s} \in \mathcal{L}(\mathcal{H})\left({ }^{n} E ; F\right)
$$


isto é, $P \in \mathcal{P}_{\mathcal{L}(\mathcal{H})}\left({ }^{n} E ; F\right)$. Dado $\varepsilon>0$, podemos tomar a representação (3.4) de $P$ de maneira que

$$
\sum_{j=1}^{k}\left\|Q_{j}\right\| \cdot\left\|R_{j}\right\|_{\mathcal{P}_{\mathcal{H}}}^{r_{j}}<(1+\varepsilon)\|P\|_{\mathcal{P}\left(\mathcal{P}_{\mathcal{H}}\right)} .
$$

Dessa forma,

$$
\begin{aligned}
\|P\|_{\mathcal{P}_{\mathcal{L}(\mathcal{H})}} & =\|\check{P}\|_{\mathcal{L}(\mathcal{H})} \leq \sum_{j=1}^{k}\left\|\check{Q}_{j}\right\| \cdot\left\|\check{R}_{j}\right\|_{\mathcal{H}}^{r_{j}} \leq \sum_{j=1}^{k} \frac{m_{j}^{m_{j}}}{m_{j} !}\left\|Q_{j}\right\| \cdot\left\|\check{R}_{j}\right\|_{\mathcal{H}}^{r_{j}} \\
& \leq \sum_{j=1}^{k} e^{m_{j}}\left\|Q_{j}\right\| \cdot\left\|\check{R}_{j}\right\|_{\mathcal{H}}^{r_{j}} \leq e^{n} \sum_{j=1}^{k}\left\|Q_{j}\right\| \cdot\left\|R_{j}\right\|_{\mathcal{P}_{\mathcal{H}}}^{r_{j}} \\
& \leq e^{n}(1+\varepsilon)\|P\|_{\mathcal{P}\left(\mathcal{P}_{\mathcal{H}}\right)} .
\end{aligned}
$$

Fazendo $\varepsilon \longrightarrow 0$, obtemos

$$
\|P\|_{\mathcal{P}_{\mathcal{L}(\mathcal{H})}} \leq e^{n}\|P\|_{\mathcal{P}\left(\mathcal{P}_{\mathcal{H}}\right)}
$$

donde segue o resultado.

\subsubsection{Ideais de composição}

O caso polinomial dos ideais de composição já foi bastante estudado, veja por exemplo [10]. Aqui o estudaremos sob a perspectiva de hiper-ideais.

Definição 3.4.4. Seja $\mathcal{I}$ um ideal de operadores. Dizemos que um polinômio $P \in$ $\mathcal{P}\left({ }^{n} E ; F\right)$ pertence a $\mathcal{I} \circ \mathcal{P}$ se existem um espaço de Banach $G$, um polinômio $Q \in \mathcal{P}\left({ }^{n} E ; G\right)$ e um operador $u \in \mathcal{I}(G ; F)$ tais que $P=u \circ Q$. Se $\left(\mathcal{I},\|\cdot\|_{\mathcal{I}}\right)$ for ideal de operadores $p$ normado, definimos ainda $\|\cdot\|_{\mathcal{I} \circ \mathcal{P}}: \mathcal{I} \circ \mathcal{P} \longrightarrow[0, \infty)$ por

$$
\|P\|_{\mathcal{I} \circ \mathcal{P}}=\inf \left\{\|u\|_{\mathcal{I}} \cdot\|Q\|\right\}
$$

onde o ínfimo é tomado sobre todas as representações de $P=u \circ Q$ com $u$ pertencente a $\mathcal{I}$.

Teorema 3.4.5. Se $\mathcal{I}$ é um ideal de operadores, então $\mathcal{I} \circ \mathcal{P}$ é um hiper-ideal de polinômios. Além disso, se $\left(\mathcal{I},\|\cdot\|_{\mathcal{I}}\right)$ for ideal p-normado (respectivamente $p$-Banach) de operadores, com $0<p \leq 1$, então $\left(\mathcal{I} \circ \mathcal{P},\|\cdot\|_{\mathcal{I} \circ \mathcal{P}}\right)$ é hiper-ideal p-normado (p-Banach) de polinômios. 
Demonstração. Dado um polinômio $P \in \mathcal{I} \circ \mathcal{P}$, podemos escrever $P=u \circ Q$, onde $G$ é um espaço de Banach, $u \in \mathcal{I}(G ; F)$ e $Q \in \mathcal{P}\left({ }^{n} E ; G\right)$. Sendo $H$ e $H_{1}$ espaços de Banach, para todos $R \in \mathcal{P}\left({ }^{m} H ; E\right)$ e $t \in \mathcal{L}\left(F ; H_{1}\right)$ temos

$$
t \circ P \circ R=t \circ(u \circ Q) \circ R=(t \circ u) \circ(Q \circ R),
$$

com $t \circ u \in \mathcal{I}\left(G ; H_{1}\right)$ e $Q \circ R \in \mathcal{P}\left({ }^{m n} H ; G\right)$. Portanto

$$
t \circ P \circ R \in \mathcal{I} \circ \mathcal{P}\left({ }^{m n} H ; H_{1}\right) \text {. }
$$

Além disso,

$$
\begin{aligned}
\|t \circ P \circ R\|_{\mathcal{I} \circ \mathcal{P}} & =\|(t \circ u) \circ(Q \circ R)\|_{\mathcal{I} \circ \mathcal{P}} \leq\|t \circ u\|_{\mathcal{I}} \cdot\|Q \circ R\| \leq\|t\| \cdot\|u\|_{\mathcal{I}} \cdot\|Q\| \cdot\|R\|^{n} \\
& \leq\|t\| \cdot\|u\|_{\mathcal{I}} \cdot\|Q\| \cdot\|R\|^{n} \cdot\left(\frac{m^{m}}{m !}\right)^{n} .
\end{aligned}
$$

Tomando o ínfimo sobre todas as representações de $P$, obtemos

$$
\|t \circ P \circ R\|_{\mathcal{I} \circ \mathcal{P}} \leq C_{m, n}\|t\| \cdot\|P\|_{\mathcal{I} \circ \mathcal{P}}\|R\|^{n} .
$$

As outras condições podem ser demonstradas com argumentos análogos aos do Teorema 2.4.1.

A relação de $\mathcal{I} \circ \mathcal{P}$ com os procedimentos da Seção 3.3 é a seguinte:

Como $\mathcal{I} \circ \mathcal{L}$ é um hiper-ideal simétrico temos que $\mathcal{P}_{\mathcal{I} \circ \mathcal{L}}=\mathcal{P}^{\mathcal{I} \circ \mathcal{L}}$. A próxima proposição compara tais classes.

Proposição 3.4.6. Para todo ideal de operadores $\mathcal{I}$,

$$
\mathcal{I} \circ \mathcal{P}=\mathcal{P}_{\mathcal{I} \circ \mathcal{L}}=\mathcal{P}^{\mathcal{I} \circ \mathcal{L}}
$$

$\operatorname{Ese}\left(\mathcal{I},\|\cdot\|_{\mathcal{I}}\right)$ é ideal p-normado, então $\|\cdot\|_{\mathcal{I} \circ \mathcal{P}}=\|\cdot\|_{\mathcal{P}_{\mathcal{I} \circ \mathcal{L}}}$.

Demonstração. A igualdade $\mathcal{I} \circ \mathcal{P}=\mathcal{P}_{\mathcal{I} \circ \mathcal{L}}$ é imediata ao observarmos que se $P=u \circ Q$, então $\check{P}=u \circ \check{Q}$; e se $A=t \circ B$, então $\widehat{A}=t \circ \widehat{B}$. De [10, Proposition 3.2] segue facilmente que o hiper-ideal $\mathcal{I} \circ \mathcal{L}$ é simétrico, logo a igualdade $\mathcal{P}_{\mathcal{I} \circ \mathcal{L}}=\mathcal{P}^{\mathcal{I} \circ \mathcal{L}}$ segue do Lema 3.3.5. A igualdade de normas segue de [10, Proposition 3.7.(b)].

Chamando de $\mathcal{P}_{\mathcal{K}}$ e $\mathcal{P}_{\mathcal{W}}$ as classes dos polinômios homogêneos compactos (a imagem da bola unitária é relativamente compacta) e fracamente compactos (a imagem da bola unitária é relativamente fracamente compacta), Ryan [48] provou que

$$
\mathcal{P}_{\mathcal{K}}=\mathcal{K} \circ \mathcal{P} \text { e } \mathcal{P}_{\mathcal{W}}=\mathcal{W} \circ \mathcal{P}
$$


Então $\mathcal{P}_{\mathcal{K}}$ e $\mathcal{P}_{\mathcal{W}}$ são hiper-ideais fechados de polinômios.

Para fornecer mais um exemplo interessante necessitamos da seguinte definição introduzida por Aron e Rueda [3] (o caso linear é devido a Sinha e Karn [53]).

Definição 3.4.7. Sejam $p, q \geq 1$ tais que $\frac{1}{p}+\frac{1}{q}=1$. Um polinômio $n$-homogêneo $P \in \mathcal{P}\left({ }^{n} E ; F\right)$ é dito $p$-compacto se existe uma sequência $\left(x_{j}\right)_{j=1}^{\infty} \in \ell_{p}(F)$ tal que

$$
P\left(B_{E}\right) \subseteq\left\{\sum_{j=1}^{\infty} \lambda_{j} x_{j}:\left(\lambda_{j}\right)_{j=1}^{\infty} \in B_{\ell_{q}}\right\} .
$$

Neste caso escrevemos $P \in \mathcal{P}_{\mathcal{K}_{p}}\left({ }^{n} E ; F\right)$. Em particular, no caso $n=1$ denotamos o ideal dos operadores lineares $p$-compactos por $\mathcal{K}_{p}(E ; F)$. Definimos ainda $\|\cdot\|_{\mathcal{P}_{\mathcal{K}_{p}}}: \mathcal{P}_{\mathcal{K}_{p}} \longrightarrow$ $[0, \infty)$ por

$$
\|P\|_{\mathcal{P}_{\mathcal{K}_{p}}}=\inf \left\{\left\|\left(x_{j}\right)_{j=1}^{\infty}\right\|_{p}:\left(x_{j}\right)_{j=1}^{\infty} \operatorname{satisfaz}(3.5)\right\}
$$

É conhecido que $\left(\mathcal{K}_{p},\|\cdot\|_{\mathcal{K}_{p}}\right)$ é um ideal de Banach de operadores (para mais detalhes veja $[52,53])$. O próximo resultado, provado em [3, Theorem 3.1], afirma entre outras coisas que $\mathcal{P}_{\mathcal{K}_{p}}=\mathcal{K}_{p} \circ \mathcal{P}$.

Proposição 3.4.8. [3, Theorem 3.1] As seguintes afirmações são equivalentes para $p \geq 1$ :

(a) $P \in \mathcal{P}_{\mathcal{K}_{p}}\left({ }^{n} E ; F\right)$.

(b) $P \in \mathcal{K}_{p} \circ \mathcal{P}\left({ }^{n} E ; F\right)$

(c) $\check{P} \in \mathcal{K}_{p} \circ \mathcal{L}\left({ }^{n} E ; F\right)$.

Neste caso, $\|P\|_{\mathcal{P}_{\mathcal{K}_{p}}}=\|P\|_{\mathcal{K}_{p} \circ \mathcal{P}}$.

Na verdade, a igualdade das normas acima segue de uma combinação de [3, Theorem 3.1] e [10, Proposition 3.7]. Da Proposição acima temos $\mathcal{P}_{\mathcal{K}_{p}}=\mathcal{K}_{p} \circ \mathcal{P}$ com igualdade de normas, e portanto $\left(\mathcal{P}_{\mathcal{K}_{p}},\|\cdot\|_{\mathcal{P}_{\mathcal{K}_{p}}}\right)$ é hiper-ideal de Banach de polinômios.

\subsubsection{Método da desigualdade}

Nesta seção transpomos para polinômios homogêneos o procedimento introduzido na Seção 2.5 para aplicações multilineares.

Definição 3.4.9. Sejam $0<p, q \leq 1, \mathcal{X}$ um $p$-funtor de sequências e $\mathcal{Y}$ um $q$-funtor de sequências. Dizemos que um polinômio $n$-homogêneo $P \in \mathcal{P}\left({ }^{n} E ; F\right)$ é $(\mathcal{X}-\mathcal{Y})$-somante 
se existe uma constante $C>0$ tal que, para toda sequência finita $\left(x_{j}\right)_{j=1}^{k}$ em $E$, tivermos

$$
\left\|\left(P\left(x_{j}\right)\right)_{j=1}^{k}\right\|_{\mathcal{Y}(F)} \leq C \cdot \sup _{R \in B_{\mathcal{P}(n E)}}\left\|\left(R\left(x_{j}\right)\right)_{j=1}^{k}\right\|_{\mathcal{X}(\mathbb{K})}
$$

Nesse caso escrevemos $P \in \mathcal{P}(\mathcal{X}-\mathcal{Y})\left({ }^{n} E ; F\right)$. Definimos ainda a função $\|\cdot\|_{\mathcal{P}(\mathcal{X}-\mathcal{Y})}: \mathcal{P}(\mathcal{X}-\mathcal{Y}) \longrightarrow[0, \infty)$ por

$$
\|P\|_{\mathcal{P}(\mathcal{X}-\mathcal{Y})}=\inf \{C>0: C \operatorname{satisfaz}(3.6)\} .
$$

Teorema 3.4.10. Sejam $0<p, q \leq 1, \mathcal{X}$ um p-funtor de sequências e $\mathcal{Y}$ um q-funtor de sequências com $\mathcal{Y}$ escalarmente dominado com respeito a $\mathcal{X}$. Então a classe $(\mathcal{P}(\mathcal{X}-\mathcal{Y}), \| \cdot$ $\left.\|_{\mathcal{P}(\mathcal{X}-\mathcal{Y})}\right)$ é um hiper-ideal q-Banach de polinômios.

Demonstração. A demonstração segue a mesma ideia do Teorema 2.5.7, usando no presente caso o Critério da Série para hiper-ideais de polinômios (Teorema 3.1.7). Como as verificações são muito similares, faremos apenas a do item (iii), que trata da desigualdade na propriedade de hiper-ideal, ponto no qual há uma diferença entre as teorias multilinear e polinomial. Para isso sejam $P \in \mathcal{P}(\mathcal{X}-\mathcal{Y})\left({ }^{n} E ; F\right), Q \in \mathcal{P}\left({ }^{m} G ; F\right)$ e $t \in \mathcal{L}(F ; H)$, com $Q \neq 0$. Dessa forma, para toda sequência finita $\left(x_{j}\right)_{j=1}^{k}$ em $G$,

$$
\begin{aligned}
\|\left(t \circ P \circ Q\left(x_{j}\right)\right)_{j=1}^{k} & \left\|_{\mathcal{Y}(H)}=\right\|\left(t\left(P\left(Q\left(x_{j}\right)\right)\right)\right)_{j=1}^{k}\left\|_{\mathcal{Y}(H)} \leq\right\| t\|\cdot\|\left(\left(P\left(Q\left(x_{j}\right)\right)\right)\right)_{j=1}^{k} \|_{\mathcal{Y}(F)} \\
& \leq\|t\| \cdot\|P\|_{\mathcal{P}(\mathcal{X}-\mathcal{Y})} \cdot \sup _{R \in B_{\mathcal{P}\left(n_{E}\right)}}\left\|\left(R\left(Q\left(x_{j}\right)\right)\right)_{j=1}^{k}\right\|_{\mathcal{X}(\mathbb{K})} \\
& =\|t\| \cdot\|P\|_{\mathcal{P}(\mathcal{X}-\mathcal{Y})} \cdot\|Q\|^{n} \cdot \sup _{R \in B_{\mathcal{P}\left(n_{E}\right)}}\left\|\left(\left(\frac{R\left(Q\left(x_{j}\right)\right)}{\|Q\|^{n}}\right)\right)_{j=1}^{k}\right\|_{\mathcal{X}(\mathbb{K})} \\
& \leq\|t\| \cdot\|P\|_{\mathcal{P}(\mathcal{X}-\mathcal{Y})} \cdot\|Q\|^{n} \sup _{S \in B_{\mathcal{P}\left({ }^{m} n_{G)}\right.}}\left\|\left(\left(S\left(x_{j}\right)\right)\right)_{j=1}^{k}\right\|_{\mathcal{X}(\mathbb{K})} \\
& \leq C_{m, n}\|t\| \cdot\|P\|_{\mathcal{P}(\mathcal{X}-\mathcal{Y})} \cdot\|Q\|^{n} \sup _{S \in B_{\mathcal{P}\left(m n_{G}\right)}}\left\|\left(\left(S\left(x_{j}\right)\right)\right)_{j=1}^{k}\right\|_{\mathcal{X}(\mathbb{K})},
\end{aligned}
$$

onde usamos a condição (iii) da Definiçao 2.5.1 na primeira desigualdade, e que se $R \in$ $B_{\mathcal{P}\left({ }^{n} E\right)}$, então $\frac{R \circ Q}{\|Q\|^{n}} \in B_{\mathcal{P}\left({ }^{m n} G\right)}$ na última desigualdade. Dessa forma

$$
t \circ P \circ Q \in \mathcal{P}(\mathcal{X}-\mathcal{Y})\left({ }^{m n} G ; H\right) \text { e }\|t \circ P \circ Q\|_{\mathcal{P}(\mathcal{X}-\mathcal{Y})} \leq C_{m, n}\|t\| \cdot\|P\|_{\mathcal{P}(\mathcal{X}-\mathcal{Y})} \cdot\|Q\|^{n}
$$

no caso em que $Q \neq 0$. Já quando $Q=0$ as afirmativas são imediatas.

O próximo objetivo é comparar $\mathcal{P}(\mathcal{X}-\mathcal{Y}) \operatorname{com} \mathcal{P}_{(\mathcal{X}-\mathcal{Y})}$ e $\mathcal{P}^{(\mathcal{X}-\mathcal{Y})}$. 
Lema 3.4.11. Sejam $0<p, q \leq 1$ e $\mathcal{X}$ um p-funtor de sequências escalarmente dominado pelo q-funtor de sequências $\mathcal{Y}$. Então o hiper-ideal $q$-Banach $\left((\mathcal{X}-\mathcal{Y}),\|\cdot\|_{(\mathcal{X}-\mathcal{Y})}\right)$ é fortemente simétrico.

Demonstração. Seja $A \in(\mathcal{X}-\mathcal{Y})\left({ }^{n} E ; F\right)$. Se mostrarmos que $A_{\sigma} \in(\mathcal{X}-\mathcal{Y})\left({ }^{n} E ; F\right)$ para toda permutação $\sigma \in S_{n}$, então podemos concluir, pelo fato de estarmos trabalhando em um espaço vetorial, que $A_{s} \in(\mathcal{X}-\mathcal{Y})\left({ }^{n} E ; F\right)$, ou seja que $(\mathcal{X}-\mathcal{Y})$ é simétrico. Para mostrarmos esse fato sejam $k \in \mathbb{N}$ e sequências finitas $\left(x_{j}^{1}\right)_{j=1}^{k}, \ldots,\left(x_{j}^{n}\right)_{j=1}^{k}$ em $E$. Temos:

$$
\begin{aligned}
\left\|\left(A_{\sigma}\left(x_{j}^{1}, \ldots, x_{j}^{n}\right)\right)_{j=1}^{k}\right\|_{\mathcal{Y}(F)} & =\left\|\left(A\left(x_{j}^{\sigma(1)}, \ldots, x_{j}^{\sigma(n)}\right)\right)_{j=1}^{k}\right\|_{\mathcal{Y}(F)} \\
& \leq\|A\|_{(\mathcal{X}-\mathcal{Y})} \cdot \sup _{T \in B_{\mathcal{L}\left(n_{E ; F}\right)}}\left\|\left(T\left(x_{j}^{\sigma(1)}, \ldots, x_{j}^{\sigma(n)}\right)\right)_{j=1}^{k}\right\|_{\mathcal{X}(\mathbb{K})} \\
& =\|A\|_{(\mathcal{X}-\mathcal{Y})} \cdot \sup _{T \in B_{\mathcal{L}\left({ }^{n} E ; F\right)}}\left\|\left(T\left(x_{j}^{1}, \ldots, x_{j}^{n}\right)\right)_{j=1}^{k}\right\|_{\mathcal{X}(\mathbb{K})},
\end{aligned}
$$

onde na última igualdade usamos que $T \in B_{\mathcal{L}\left({ }^{n} E ; F\right)}$ se, e somente se, $T_{\sigma} \in B_{\mathcal{L}\left({ }^{n} E ; F\right)}$. Dessa forma $A_{\sigma} \in(\mathcal{X}-\mathcal{Y})\left({ }^{n} E ; F\right)$ e $\left\|A_{\sigma}\right\|_{(\mathcal{X}-\mathcal{Y})} \leq\|A\|_{(\mathcal{X}-\mathcal{Y})}$.

Agora usando que $A_{\sigma} \in(\mathcal{X}-\mathcal{Y})\left({ }^{n} E ; F\right)$ para toda permutação $\sigma \in S_{n}$ e que $A=$ $A_{\sigma \circ \sigma^{-1}}=\left(A_{\sigma}\right)_{\sigma^{-1}}$, pela desigualdade provada no parágrafo anterior temos

$$
\|A\|_{(\mathcal{X}-\mathcal{Y})}=\left\|\left(A_{\sigma}\right)_{\sigma^{-1}}\right\|_{(\mathcal{X}-\mathcal{Y})} \leq\left\|A_{\sigma}\right\|_{(\mathcal{X}-\mathcal{Y})} .
$$

Portanto $\|A\|_{(\mathcal{X}-\mathcal{Y})}=\left\|A_{\sigma}\right\|_{(\mathcal{X}-\mathcal{Y})}$ e $\left((\mathcal{X}-\mathcal{Y}),\|\cdot\|_{(\mathcal{X}-\mathcal{Y})}\right)$ é fortemente simétrico.

Proposição 3.4.12. Sejam $0<p, q \leq 1, \mathcal{X}$ um p-funtor de sequências e $\mathcal{Y}$ um q-funtor de sequências com $\mathcal{Y}$ escalarmente dominado com respeito a $\mathcal{X}$. Então

$$
\mathcal{P}_{(\mathcal{X}-\mathcal{Y})}=\mathcal{P}^{(\mathcal{X}-\mathcal{Y})} \subseteq \mathcal{P}(\mathcal{X}-\mathcal{Y}) e\|\cdot\|_{\mathcal{P}(\mathcal{X}-\mathcal{Y})} \leq\|\cdot\|_{\mathcal{P}^{(\mathcal{X}-\mathcal{Y})}} \leq\|\cdot\|_{\mathcal{P}_{(\mathcal{X}-\mathcal{Y})}}
$$

Demonstração. A igualdade $\mathcal{P}_{(\mathcal{X}-\mathcal{Y})}=\mathcal{P}^{(\mathcal{X}-\mathcal{Y})}$ segue do Lema 3.4.11 e da Proposição 3.3.6. Seja $P \in \mathcal{P}^{(\mathcal{X}-\mathcal{Y})}\left({ }^{n} E ; F\right)$. Por definição existe $A \in(\mathcal{X}-\mathcal{Y})\left({ }^{n} E ; F\right)$ tal que $\widehat{A}=P$. Para quaisquer sequências finitas $\left(x_{j}^{1}\right)_{j=1}^{k}, \ldots,\left(x_{j}^{n}\right)_{j=1}^{k}$ em $E$, temos

$$
\left\|\left(A\left(x_{j}^{1}, \ldots, x_{j}^{n}\right)\right)_{j=1}^{k}\right\|_{\mathcal{Y}(F)} \leq\|A\|_{(\mathcal{X}-\mathcal{Y})} \cdot \sup _{T \in B_{\mathcal{L}\left(n_{E}\right)}}\left\|\left(T\left(x_{j}^{1}, \ldots, x_{j}^{n}\right)\right)_{j=1}^{k}\right\|_{\mathcal{X}(\mathbb{K})} .
$$

Dados $x_{1}, \ldots, x_{k} \in E$, fazendo $x_{j}^{1}, \ldots, x_{j}^{n}:=x_{j}$, para todo $j \in \mathbb{N}$,

$$
\begin{aligned}
\left\|\left(P\left(x_{j}\right)\right)_{j=1}^{k}\right\|_{\mathcal{Y}(F)} & =\left\|\left(A\left(x_{j}, \ldots, x_{j}\right)\right)_{j=1}^{k}\right\|_{\mathcal{Y}(F)} \\
& \leq\|A\|_{(\mathcal{X}-\mathcal{Y})} \cdot \sup _{T \in B_{\mathcal{L}\left({ }^{n}\right)}}\left\|\left(T\left(x_{j}, \ldots, x_{j}\right)\right)_{j=1}^{k}\right\|_{\mathcal{X}(\mathbb{K})} \\
& =\|A\|_{(\mathcal{X}-\mathcal{Y})} \cdot \sup _{T \in B_{\mathcal{L}\left({ }^{n} E\right)}}\left\|\left(\widehat{T}\left(x_{j}\right)\right)_{j=1}^{k}\right\|_{\mathcal{X}(\mathbb{K})} \\
& \leq\|A\|_{(\mathcal{X}-\mathcal{Y})} \cdot \sup _{R \in B_{\mathcal{P}\left(n_{E}\right)}}\left\|\left(R\left(x_{j}\right)\right)_{j=1}^{k}\right\|_{\mathcal{X}(\mathbb{K})},
\end{aligned}
$$


onde a última desigualdade segue do fato de que se $T \in B_{\mathcal{L}\left({ }^{n} E\right)}$, então $\widehat{T} \in B_{\mathcal{P}\left({ }^{n} E\right)}$. Portanto $P \in \mathcal{P}(\mathcal{X}-\mathcal{Y})$ e $\|P\|_{\mathcal{P}(\mathcal{X}-\mathcal{Y})} \leq\|A\|_{(\mathcal{X}-\mathcal{Y})}$. Tomando o ínfimo sobre todas as aplicações $A$ em $(\mathcal{X}-\mathcal{Y})$ tais que $\widehat{A}=P$, tem-se $\|P\|_{\mathcal{P}(\mathcal{X}-\mathcal{Y})} \leq\|P\|_{\left.\mathcal{P}^{(\mathcal{X}}-\mathcal{Y}\right)}$. A desigualdade restante segue de (3.1).

Exemplo 3.4.13. Vejamos que a inclusão apresentada na proposição anterior é estrita. Para isso consideremos os funtores de sequências $\mathcal{X}=\mathcal{Y}=\ell_{1}(\cdot)$ e o polinômio 2-homogêneo

$$
P: \ell_{2} \rightarrow \ell_{2} \widehat{\otimes}_{\pi} \ell_{2}, P(x)=x \otimes x .
$$

Traduzindo o que está provado em [26, Example 3.4] para a nossa notação, temos $P \in$ $\mathcal{P}\left(\ell_{1}(\cdot)-\ell_{1}(\cdot)\right)\left({ }^{2} \ell_{2} ; \ell_{2} \widehat{\otimes}_{\pi} \ell_{2}\right)$, mas $\check{P} \notin\left(\ell_{1}(\cdot)-\ell_{1}(\cdot)\right)\left({ }^{2} \ell_{2} ; \ell_{2} \widehat{\otimes}_{\pi} \ell_{2}\right)$, onde

$$
\check{P}(x, y)=\frac{x \otimes y+y \otimes x}{2} .
$$

$\operatorname{Logo} \mathcal{P}_{\left(\ell_{1}(\cdot)-\ell_{1}(\cdot)\right)} \varsubsetneqq \mathcal{P}\left(\ell_{1}(\cdot)-\ell_{1}(\cdot)\right)$.

Como caso particular da Definição 3.4 .9 obtemos a seguinte classe de polinômios, introduzida por V. Dimant em [26, Definition 3.1]:

Definição 3.4.14. Seja $p>0$. Um polinômio $P \in \mathcal{P}\left({ }^{n} E ; F\right)$ é fortemente $p$-somante se existe uma constante $C>0$ tal que, para qualquer sequência finita $\left(x_{j}\right)_{j=1}^{k}$ em $E$, tem-se

$$
\left(\sum_{j=1}^{k}\left\|P\left(x_{j}\right)\right\|^{p}\right)^{1 / p} \leq C \cdot \sup _{q \in B_{\mathcal{P}\left(n_{E}\right)}}\left(\sum_{j=1}^{k}\left|q\left(x_{j}\right)\right|^{p}\right)^{1 / p}
$$

Nesse caso escrevemos $P \in \mathcal{P}_{s s}^{p}\left({ }^{n} E ; F\right)$. Definimos ainda $\|\cdot\|_{\mathcal{P}_{s s}^{p}}: \mathcal{P}_{s s}^{p} \longrightarrow[0, \infty)$ por

$$
\|P\|_{\mathcal{P}_{s s}^{p}}=\inf \{C: C \text { satisfaz }(3.7)\} .
$$

Como esperado temos a:

Proposição 3.4.15. Seja $0<p<\infty$. Então:

(a) $\left(\mathcal{P}_{s s}^{p},\|\cdot\|_{s s, p}\right)$ é um hiper-ideal $p$-Banach de polinômios se $0<p<1$.

(b) $\left(\mathcal{P}_{s s}^{p},\|\cdot\|_{s s, p}\right)$ é hiper-ideal de Banach de polinômios se $p \geq 1$.

Demonstração. A demonstração é análoga à da Proposição 2.5.13. 


\subsubsection{Método da $\mathcal{I}$-limitação}

Finalizamos a tese com o caso polinomial do método da $\mathcal{I}$-limitação, que foi primeiramente apresentado em [4] e que é a transposição natural do método visto na Seção 2.6 .

Definição 3.4.16. Sejam $P \in \mathcal{P}\left({ }^{n} E ; F\right)$ e $\mathcal{I}$ um ideal de operadores. Dizemos que $P$ é $\mathcal{I}$-limitado se $P\left(B_{E}\right) \in C_{\mathcal{I}}(F)$, ou seja se existem um espaço de Banach $H$ e um operador $u \in \mathcal{I}(H ; F)$ tal que

$$
P\left(B_{E}\right) \subseteq u\left(B_{H}\right)
$$

Nesse caso escrevemos $P \in \mathcal{P}_{\langle\mathcal{I}\rangle}\left({ }^{n} E ; F\right)$. Definimos ainda, a partir de uma $p$-norma $\|\cdot\|_{\mathcal{I}}$ em $\mathcal{I}$, uma correspondência $\|\cdot\|_{\mathcal{P}_{\langle\mathcal{I}\rangle}}: \mathcal{P}_{\langle\mathcal{I}\rangle} \longrightarrow[0, \infty)$ dada por

$$
\|P\|_{\mathcal{P}_{\langle\mathcal{I}\rangle}}=\inf \left\{\|u\|_{\mathcal{I}} ; u \text { satisfaz }(3.8)\right\}
$$

Para a diferença em relação à notação original de [4], veja a Observação 2.6.3.

Teorema 3.4.17. Sejam $0<p \leq 1$ e $\left(\mathcal{I},\|\cdot\|_{\mathcal{I}}\right)$ um ideal $p$-Banach de operadores. Então $\left(\mathcal{P}_{\langle\mathcal{I}\rangle},\|\cdot\|_{\mathcal{P}_{\langle\mathcal{I}\rangle}}\right)$ é um hiper-ideal p-Banach de polinômios.

Demonstração. A demonstração, usando o Critério da Série para polinômios (Teorema 3.1.7), é análoga à do Teorema 2.6.4. Tamanha é sua semelhança que, sendo $t \in \mathcal{L}(F ; H)$, $P \in \mathcal{P}_{\langle\mathcal{I}\rangle}\left({ }^{n} E ; F\right)$ e $Q \in \mathcal{P}\left({ }^{m} E ; F\right)$, podemos majorar $\|t \circ P \circ Q\|_{\mathcal{P}_{\langle\mathcal{I}\rangle}}$ por $\|t\| \cdot\|P\|_{\mathcal{P}_{\langle\mathcal{I}\rangle}} \cdot\|Q\|^{n}$ omitindo a constante $C_{n, m}$. Isso também se deve ao fato de que o cálculo da $p$-norma é feito com respeito à $p$-norma $\|\cdot\|_{\mathcal{I}}$ do ideal de operadores.

Exemplo 3.4.18. As classes de polinômios p-compactos, compactos e fracamente compactos são exemplos de hiper-ideais obtidos através do método da $\mathcal{I}$-limitação. Basta tomar $\mathcal{I}$ como sendo o ideal de operadores $p$-compactos, compactos e fracamente compactos. Mais explicitamente, $\mathcal{P}_{\left\langle\mathcal{K}_{p}\right\rangle}$ coincide com a classe dos polinômios $p$-compactos, $\mathcal{P}_{\langle\mathcal{K}\rangle}$ coincide com a classe dos polinômios compactos e $\mathcal{P}\langle\mathcal{W}\rangle$ coincide com a classe dos polinômios fracamente compactos. A demonstração desses fatos é análoga à descrita no Exemplo 2.6.6 e na Observação 2.6.7.

O próximo passo é comparar a classe da Definição 3.4 .16 com as classes obtidas a

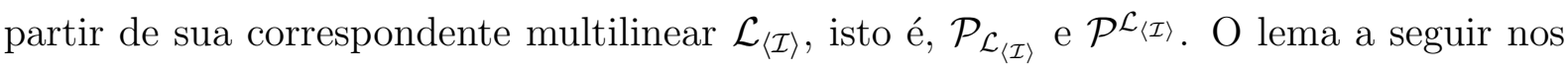
auxilia nesse sentido. 
Lema 3.4.19. Sejam $0<p \leq 1 e\left(\mathcal{I},\|\cdot\|_{\mathcal{I}}\right)$ um ideal p-Banach de operadores. O hiperideal $\left(\mathcal{L}_{\langle\mathcal{I}\rangle},\|\cdot\|_{\mathcal{L}_{\langle\mathcal{I}\rangle}}\right)$ é fortemente simétrico.

Demonstração. Seja $A \in \mathcal{L}_{\langle\mathcal{I}\rangle}\left({ }^{n} E ; F\right)$. Então $A\left(B_{E} \times \cdots \times B_{E}\right) \in C_{\mathcal{I}}(F)$, ou seja existem um espaço de Banach $H$ e um operador linear $u \in \mathcal{I}(H ; F)$ tal que $A\left(B_{E} \times \cdots \times B_{E}\right) \subseteq$ $u\left(B_{H}\right)$. Como $A_{\sigma}\left(x_{1}, \ldots, x_{n}\right)=A\left(x_{\sigma(1)}, \ldots, x_{\sigma(n)}\right)$ para quaisquer $x_{1}, \ldots, x_{n} \in E$ e $\sigma \in$ $S_{n}$, segue imediatamente que

$$
A_{\sigma}\left(B_{E} \times \cdots \times B_{E}\right) \subseteq u\left(B_{H}\right)
$$

ou seja $A_{\sigma}\left(B_{E} \times \cdots \times B_{E}\right) \in C_{\mathcal{I}}(F)$. Logo, para toda $\sigma \in S_{n}, A_{\sigma} \in \mathcal{L}_{\langle\mathcal{I}\rangle}\left({ }^{n} E ; F\right)$; e pela arbitrariedade de $u$ obtemos

$$
\left\|A_{\sigma}\right\|_{\mathcal{L}_{\langle\mathcal{I}\rangle}} \leq\|A\|_{\mathcal{L}_{\langle\mathcal{I}\rangle}} .
$$

Agora, usando o que acabamos de mostrar e que $A=\left(A_{\sigma}\right)_{\sigma^{-1}}$, obtemos

$$
\|A\|_{\mathcal{L}_{\langle\mathcal{I}\rangle}}=\left\|\left(A_{\sigma}\right)_{\sigma^{-1}}\right\|_{\mathcal{L}_{\langle\mathcal{I}\rangle}} \leq\left\|A_{\sigma}\right\|_{\mathcal{L}_{\langle\mathcal{I}\rangle}}
$$

e a igualdade desejada segue. Portanto $\left(\mathcal{L}_{\langle\mathcal{I}\rangle},\|\cdot\|_{\mathcal{L}_{\langle\mathcal{I}\rangle}}\right)$ é fortemente simétrico.

Chegamos então ao último resultado desta seção:

Proposição 3.4.20. Sejam $0<p \leq 1 e\left(\mathcal{I},\|\cdot\|_{\mathcal{I}}\right)$ um ideal $p$-Banach de operadores. Então $\mathcal{P}_{\langle\mathcal{I}\rangle}=\mathcal{P}_{\mathcal{L}_{\langle\mathcal{I}\rangle}}=\mathcal{P}^{\mathcal{L}_{\langle\mathcal{I}\rangle} \text { e valem as desigualdades }}$

$$
\|P\|_{\mathcal{P}^{\mathcal{L}}\langle\mathcal{I}\rangle} \leq\|P\|_{\mathcal{P}_{\mathcal{L}_{\langle\mathcal{I}\rangle}}} \leq(n !)^{\frac{1}{p}-1}\|P\|_{\mathcal{P}^{\mathcal{L}}\langle\mathcal{I}\rangle}
$$

$e$

$$
\|P\|_{\mathcal{P}_{\langle\mathcal{I}\rangle}} \leq\|P\|_{\mathcal{P}_{\mathcal{L}_{\langle\mathcal{I}\rangle}}} \leq \frac{n^{n}}{n !}\|P\|_{\mathcal{P}_{\langle\mathcal{I}\rangle}}
$$

para todo $P \in \mathcal{P}_{\langle\mathcal{I}\rangle}\left({ }^{n} E ; F\right)$.

Demonstração. Como vimos no Lema 3.4.19, $\left(\mathcal{L}_{\langle\mathcal{I}\rangle},\|\cdot\|_{\mathcal{L}_{\langle\mathcal{I}\rangle}}\right)$ é um hiper-ideal $p$-Banach fortemente simétrico. Então, segue diretamente da Proposição 3.3 .6 que $\mathcal{P}_{\mathcal{L}_{\langle\mathcal{I}\rangle}}=\mathcal{P}^{\mathcal{L}_{\langle\mathcal{I}\rangle}}$ e

$$
\|P\|_{\mathcal{P}^{\mathcal{L}}\langle\mathcal{I}\rangle} \leq\|P\|_{\mathcal{P}^{\mathcal{L}}\langle\mathcal{I}\rangle} \leq(n !)^{\frac{1}{p}-1}\|P\|_{\mathcal{P}^{\mathcal{L}}\langle\mathcal{I}\rangle},
$$

para todo $P \in \mathcal{P}_{\mathcal{L}_{\langle\mathcal{I}\rangle}}\left({ }^{n} E ; F\right)$.

Agora mostremos que $\mathcal{P}_{\langle\mathcal{I}\rangle}=\mathcal{P}_{\mathcal{L}_{\langle\mathcal{I}\rangle}}$ e que vale a desigualdade inversa. Se $P \in$ $\mathcal{P}_{\langle\mathcal{I}\rangle}\left({ }^{n} E ; F\right)$, então existem espaços de Banach $H$ e um operador $u \in \mathcal{I}(H ; F)$ tais que 
$P\left(B_{E}\right) \subseteq u\left(B_{H}\right)$. Dados $x_{1}, \ldots, x_{n} \in B_{E}$, para cada escolha de sinais $\varepsilon_{1}, \ldots, \varepsilon_{n}$, é claro que $\frac{\varepsilon_{1} x_{1}+\cdots+\varepsilon_{n} x_{n}}{n} \in B_{E}$. Existe então $z_{\varepsilon_{1}, \ldots, \varepsilon_{n}} \in B_{H}$ tal que

$$
P\left(\frac{\varepsilon_{1} x_{1}+\cdots+\varepsilon_{n} x_{n}}{n}\right)=u\left(z_{\varepsilon_{1}, \ldots, \varepsilon_{n}}\right)
$$

Pela Fórmula da Polarização (Proposição 1.2.8) obtemos

$$
\begin{aligned}
\check{P}\left(x_{1}, \ldots, x_{n}\right) & =\frac{1}{n ! 2^{n}} \sum_{\varepsilon_{j}= \pm 1} \varepsilon_{1} \cdots \varepsilon_{n} P\left(\varepsilon_{1} x_{1}+\cdots+\varepsilon_{n} x_{n}\right) \\
& =\frac{1}{n ! 2^{n}} \sum_{\varepsilon_{j}= \pm 1} \varepsilon_{1} \cdots \varepsilon_{n} n^{n} P\left(\frac{\varepsilon_{1} x_{1}+\cdots+\varepsilon_{n} x_{n}}{n}\right) \\
& =\frac{n^{n}}{n ! 2^{n}} \sum_{\varepsilon_{j}= \pm 1} \varepsilon_{1} \cdots \varepsilon_{n} u\left(z_{\varepsilon_{1}, \ldots, \varepsilon_{n}}\right)=\frac{n^{n}}{n !} u(w),
\end{aligned}
$$

onde $w=\frac{1}{2^{n}} \sum_{\varepsilon_{j}= \pm 1} \varepsilon_{1} \cdots \varepsilon_{n} z_{\varepsilon_{1}, \ldots, \varepsilon_{n}}$. Como $w \in B_{H}$ e $\frac{n^{n}}{n !} u \in \mathcal{I}(H ; F)$, concluímos que

$$
\check{P}\left(B_{E} \times \cdots \times B_{E}\right) \subseteq \frac{n^{n}}{n !} u\left(B_{H}\right)
$$

isto é, $\check{P} \in \mathcal{L}_{\langle\mathcal{I}\rangle}\left({ }^{n} E ; F\right)$. Além disso,

$$
\|P\|_{\mathcal{P}_{\mathcal{L}_{\langle\mathcal{I}\rangle}}}=\|\check{P}\|_{\mathcal{L}_{\langle\mathcal{I}\rangle}} \leq\left\|\frac{n^{n}}{n !} u\right\|_{\mathcal{I}}=\frac{n^{n}}{n !} \cdot\|u\|_{\mathcal{I}}
$$

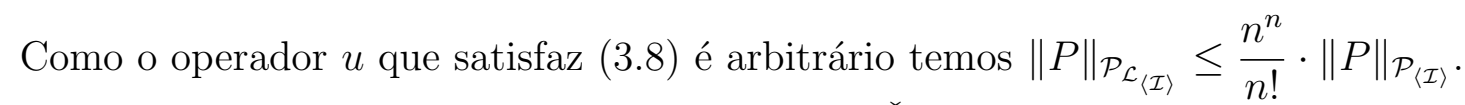

Reciprocamente, se $P \in \mathcal{P}_{\mathcal{L}_{\langle\mathcal{I}\rangle}}\left({ }^{n} E ; F\right)$, então $\check{P} \in \mathcal{L}_{\langle\mathcal{I}\rangle}\left({ }^{n} E ; F\right)$. Disso segue que

$$
\check{P}\left(B_{E} \times \cdots \times B_{E}\right) \subseteq u\left(B_{H}\right)
$$

para algum operador $u \in \mathcal{I}(H ; F)$. Como $P\left(B_{E}\right) \subseteq \check{P}\left(B_{E} \times \cdots \times B_{E}\right)$, temos

$$
P\left(B_{E}\right) \subseteq u\left(B_{H}\right)
$$

donde decorre que $P \in \mathcal{P}_{\langle\mathcal{I}\rangle}\left({ }^{n} E ; F\right)$. Além disso, novamente pela arbitrariedade de $u$ e

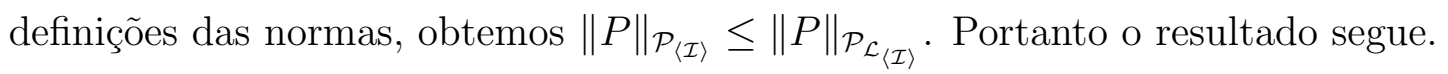




\section{Referências Bibliográficas}

[1] Alves, T. R., Polinômios Dominados entre Espaços de Banach, Dissertação de Mestrado, FAMAT, UFU, 2011.

[2] Aron, R.; Dimant, V., Sets of weak sequential continuity for polynomials, Indagationes Mathematicae, vol. 13(3) (2002), pp. 287-299

[3] Aron, R. M.; Rueda, P., p-Compact homogeneous polynomials from an ideal point of view, Contemporary Mathematics, vol. 547 (2011), pp. 61-71.

[4] Aron, R. M.; Rueda, P., Ideals of homogeneous polynomials, Publications of the Research Institute for Mathematical Sciences Kyoto Universty, vol. 46 (2012), pp. 957-969.

[5] Aron, R. M.; RuedA, P., I-bounded holomorphic functions, Preprint.

[6] Bertoloto, F.; Botelho, G.; Fávaro, V. V.; Jatobá, A., Hypercyclicity of convolution operators on spaces of entire functions, Annales de l'Institut Fourier (Grenoble), vol. 63 (2013), no. 4, pp. 1263-1283.

[7] Botelho, G., Ideals of polynomials generated by weakly compact operators, Note di Matematica, vol. 25 (2005), pp. 69-102.

[8] Botelho, G.; Braunss, H.A.; Junek, H.; Pellegrino, D., Holomorphy types and ideals of multilinear mappings, Studia Mathematica, vol. 177 (2006), no. 1, pp. 43-65.

[9] Botelho, G.; Pellegrino, D., Two new properties of ideals of polynomials and applications, Indagationes Mathematicae (N.S.), vol. 16 (2005), no. 2, pp. 157-169.

[10] Botelho, G.; Pellegrino, D.; Rueda, P., On composition ideals of multilinear mappings and homogeneous polynomials, Publications of the Resesarch Institute for Mathematical Sciences, vol. 43 (2007), pp. 1139-1155. 
[11] Botelho G.; Pellegrino, D.; Rueda P., On Pietsch measures for summing operators and dominated polynomials, Linear and Multilinear Algebra, vol. 62 (2014), pp. 860-874.

[12] Botelho, G.; Pellegrino, D.; Teixeira, E., Fundamentos de Análise Funcional, Sociedade Brasileira de Matemática, 2012.

[13] Botelho G.; Rueda P., The Schur property on projective and injective tensor products, Proceedings of the American Mathematical Society, vol. 137 (2009), pp. 219-225.

[14] Boyd, C.; Ryan, R.A., Bounded weak continuity of homogeneous polynomials at the origin, Archiv der Mathematik, vol. 71 (1998), pp. 211-218

[15] Braunss, H.-A., Ideale Multilinearer Abbildungen und Räume holomorpher Funktionen, Dissertation (A), Pädagogische Hochschule 'Karl Liebknecht', Potsdam, 1984.

[16] Braunss,H.-A.; Junek, H., Ideals of Polynomials and Multilinear Mappings, unpublished notes.

[17] Campos, J., Cohen and multiple Cohen strongly summing multilinear operators, Linear and Multilinear Algebra, vol. 62 (2014), no. 3, pp. 322-346.

[18] Carando, D.; Dimant, V.; Muro, S., Hypercyclic convolution operators on Fréchet spaces of analytic functions, Journal of Mathematical Analysis and Applications, vol. 336 (2007), no. 2, pp. 1324-1340.

[19] Carando, D.; Dimant, V.; Muro, S., Coherent sequences of polynomial ideals on Banach spaces, Mathematische Nachrichten, vol. 282 (2009), no. 8, pp. 1111-1133.

[20] Castillo, J.M.F.; García, R.; Gonzalo, R., Banach spaces in which all multilinear forms are weakly sequentially continuous, Studia Mathematica, vol. 136(2) (1999), pp. 121-145

[21] Defant, A.; Floret, K., Tensor Norms and Operator Ideals, North-Holland, 1992.

[22] Defant, A.; García, D.; Maestre, M.; PÉrez-García, D., Bohr's strip for vector valued Dirichlet series, Mathematische Annalen, vol. 342 (2008), pp. 533-555.

[23] Defant, A.; Popa, D.; Schwarting, U., Coordinatewise multiple summing operators in Banach spaces, Journal of Functional Analysis, vol. 259 (2010), no.1, pp. 220-242. 
[24] Diestel, D.; Jarchow, H.; Pietsch, A., Operator Ideals, in: Handbook of the Geometry of Banach Spaces, Volume 1, pp. 437-496, North-Holland, 2001.

[25] Diestel, J.; Jarchow, H.; Tonge, A., Absolutely Summing Operators, Cambridge University Press, New York, 1995.

[26] Dimant, V., Strongly p-summing multilinear operators, Journal of Mathematical Analysis and Applications, vol. 278 (2003), pp. 182-193.

[27] Dineen, S., Complex Analysis in Locally Convex Spaces, North-Holland Mathematics Studies 57, 1981.

[28] FÁvaro, V.V.; JatobÁ, A., Holomorphy types and spaces of entire functions of bounded type on Banach spaces, Czechoslovak Mathematical Journal, vol. 59(134) (2009), no. 4, pp. 909-927.

[29] FÁvaro, V.V.; JatobÁ, A., Holomorphy types and the Fourier-Borel transform between spaces of entire functions of a given type and order defined on Banach spaces, Mathematica Scandinavica, vol. 110 (2012), no. 1, pp. 111-139.

[30] Floret, K., On ideals of n-homogeneous polynomials on Banach spaces, Topological Algebras with Applications to Differential Geometry and Mathematical Physics, Athens (1999), pp. 19-38.

[31] Floret, K; GarcíA, D., On ideals of polynomials and multilinear mappings between Banach spaces, Archiv der Mathematik (Basel), vol. 81 (2003), pp. 300-308.

[32] GeIss, S., Ideale Multilinearer Abbildungen, Diplomarbeit, Universität Jena, 1985.

[33] GonzÁlez, M.; GutiÉrrez J. M., Surjective factorization of holomorphic mappings, Commentationes Mathematicae Universitatis Carolinae, vol. 41 (2000), pp. 469-476.

[34] Kalton, N., Quasi-Banach spaces, in: Handbook of the Geometry of Banach Spaces, Volume 2, pp. 1099-1130, North-Holland, 2003.

[35] Matos, M., On multilinear mappings of nuclear type, Revista Matematica de la Universidad Complutense de Madrid, vol. 6 (1993), no.1 pp. 61-81.

[36] Megginson, R. E., An Introduction to Banach Space Theory, Graduate Texts in Mathematics 183, Springer-Verlag, New York, 1998. 
[37] Montanaro, A., Some applications of hypercontractive inequalities in quantum information theory, Journal of Mathematical Physics, vol. 53 (2012), no.12, 122206, $15 \mathrm{pp}$.

[38] Mujica, J., Complex Analysis in Banach Spaces, Dover Publications, Mineola, New York, 2010.

[39] Pellegrino, D.; Ribeiro, J., On multi-ideals and polynomial ideals of Banach spaces: a new approach to coherence and compatibility, Monatshefte für Mathematik, vol. 173 (2014), no. 3, pp. 379-415.

[40] Peeczyński, A., On weakly compact polynomial operators on B-spaces with Dunford-Pettis Property, Bulletin of the Polish Academy of Sciences Mathematics, vol. 11 (1963), pp. 371-377.

[41] PÉrez-García, D., Operadores Multilineales Absolutamente Sumantes, Tesina, Departamento de Análisis Matemático, Facultad de Ciencias Matemáticas, Universidad Complutense de Madrid, 2002.

[42] Pérez-García, D.; Wolf, M. M.; Palazuelos, C.; Villanueva, I.; Junge, M., Unbounded violation of tripartite Bell inequalities, Communications in Mathematical Physics, vol. 279 (2008), pp. 455-486.

[43] Pietsch, A., Operator Ideals, North Holland, 1980.

[44] Pietsch, A., Ideals of multilinear functionals, Proceedings of the Second International Conference on Operator Algebras, Ideals and Their Applications in Theoretical Physics, Leipzig Teubner Texte Math, vol. 62 (1983), pp. 185-199.

[45] Polac, L. G., O Adjunto de um Polinômio Homogêneo Contínuo entre Espaços de Banach, Dissertação de Mestrado, FAMAT, UFU, 2013.

[46] PopA, D., Nuclear multilinear operators with respect to a partition, Rendiconti del Circolo Matematico di Palermo, vol. 61 (2012), pp. 307-319.

[47] PopA, D., Composition results for strongly summing and dominated multilinear operators, Central European Journal of Mathematics, vol. 12 (2014), pp. 1433-1446.

[48] Ryan, R. A., Applications of Topological Tensor Products to Infinite Dimensional Holomorphy, Thesis, Trinity College Dublin, 1980. 
[49] Ryan, R. A., Introduction to Tensor Products of Banach Spaces, Springer-Verlag, 2002.

[50] Serrano-Rodríguez, D.M., Absolutely $\gamma$-summing multilinear operators, Linear Algebra and its Applications, vol. 439 (2013), pp. 4110-4118.

[51] Silva, A. R., Linearização de Aplicações Multilineares Contínuas entre Espaços de Banach e Multi-Ideais de Composição, Dissertação de Mestrado, FAMAT, UFU, 2010.

[52] Silva, R. C., Operadores p-Compactos e a Propriedade de p-Aproximação, Dissertação de Mestrado, IME, USP, 2013.

[53] Sinha, D.P.; Karn, A.K., Compact operators whose adjoints factor through subspaces of $\ell_{p}$, Studia Mathematica, vol. 150 (2002), pp. 17-33.

[54] Stephani, I., Generating systems of sets and quotients of surjective operator ideals, Mathematische Nachrichten, vol. 99 (1980), pp. 13-27. 